\title{
ORPHANED IN THE ARCHIVE: THE LIBRARY AND ARCHIVES CANADA 28MM FILM
} COLLECTION

\author{
by \\ Andrew H. Watts \\ A thesis \\ presented to Ryerson University \\ in partial fulfillment of the \\ requirements for the degree of \\ Master of Arts \\ in the program of
}

BA Cinema Studies, University of Calgary, Calgary, Alberta, Canada, 2017

Film and Photography Preservation and Collections Management

Toronto, Ontario, Canada, 2019

(C) Andrew Watts, 2019 


\section{AUTHOR'S DECLARATION FOR ELECTRONIC SUBMISSION OF A THESIS}

I hereby declare that I am the sole author of this thesis. This is a true copy of the thesis, including any required final revisions, as accepted by my examiners.

I authorize Ryerson University to lend this thesis to other institutions or individuals for the purpose of scholarly research.

I further authorize Ryerson University to reproduce this thesis by photocopying or by other means, in total or in part, at the request of other institutions or individuals for the purpose of scholarly research.

I understand that my thesis may be made electronically available to the public. 
Orphaned in the Archive: The Library and Archives Canada 28mm Film Collection

Master of Arts, Film and Photography Preservation and Collections Management, 2019 Andrew H. Watts

Ryerson University, Toronto, Canada

\begin{abstract}
This thesis provides a spreadsheet of the $28 \mathrm{~mm}$ films held at Library and Archives Canada (LAC). Consisting of 1,135 film spread over 34 fonds, the collection is one of the largest of both $28 \mathrm{~mm}$ and government-produced films in the world. Despite LAC promoting the collection as an important part of its audiovisual holdings, current public-facing search options make it difficult for researchers to retrieve or view the $28 \mathrm{~mm}$ films. With LAC's mandate in mind, and in order to provide access to these materials, the thesis aims to fill a gap by creating a spreadsheet that acts as an easy access point to the collection. Besides producing a comprehensive list of films the thesis briefly traces the history of $28 \mathrm{~mm}$ film, its importance to early Canadian film, highlights particular films of interest and suggests paths for further research.
\end{abstract}




\section{Acknowledgements}

I would like to thank my first reader, Marta Braun, and my second reader, Christina Stewart, for their direction, support, and patience. Their suggestions and criticisms pushed me to work harder and strive to produce the best work I could. Both instilled confidence in my work and me when, at times, it was lacking. I would also like to thank my supervisor at Library and Archives Canada, Steve Moore, for allowing me the freedom to explore and develop my topic while being extremely supportive along the way.

Thanks to Pascal LeBlond for allowing me to complete my residency at Library and Archives Canada, Paul Gordon, for pushing me to explore the $28 \mathrm{~mm}$ collection and granting me access to the films, and finally Caroline Forcier-Holloway, who listened patiently to my numerous subject ideas. Finally, I would like to thank my family and classmates for their support throughout my time in the F+PPCM program. 


\section{TABLE OF CONTENTS}

ABSTRACT iii

LIST OF FIGURES - vi

$\begin{array}{ll}\text { INTRODUCTION } & 1\end{array}$

LITERATURE REVIEW

CANADIAN FILM HISTORY BEFORE 1939

PATHÉ 28MM AND NONTHEATRICAL FILM $r$

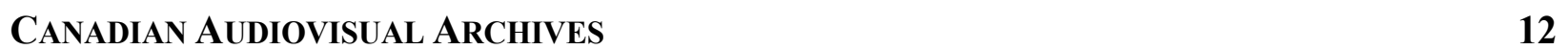

CHAPTER 1: THE RISE OF NONTHEATRICAL CINEMA ON 28MM FILM 14

CHAPTER 2: EARLY CANADIAN FILM AND THE INTRODUCTION OF 28MM FILM (1912-1917) 18

CHAPTER 3: THE 28MM COLLECTION AT LIBRARY AND ARCHIVES CANADA 24

CHAPTER 4: THE SPREAD SHEET: 28MM FILM AT LAC 27

CHAPTER 5: RESULTS

I. ThE STATISTICS

II. INTERESTING DISCOVERIES

III. THE INTERSECTION BETWEEN AMATEUR EQUIPMENT AND PROFESSIONAL INDUSTRY 37

$\begin{array}{lr}\text { CONCLUSION } & 38\end{array}$

APPENDIX A: LIBRARY AND ARCHIVES CANADA COMPREHENSIVE 28MM FILM LIST 39

$\begin{array}{ll}\text { BIBLIOGRAPHY } & 99\end{array}$ 


\section{List of Figures}

Figure 1. Screenshot of LAC Film, Video and Sound search function, from LAC website.......3

Figure 2. Ontario Motion Picture Bureau (OMPB) original $28 \mathrm{~mm}$ film can....................30

Figure 3. Cream Grading in Saskatchewan printed on Eastman Kodak 28mm stock with edge

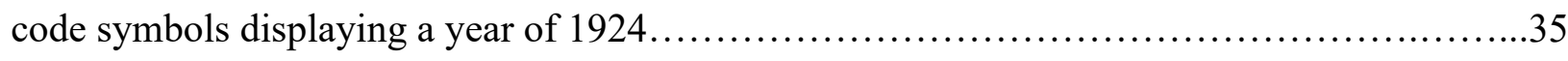

Figure 4. Side-by-side of [Dairy Farming Industry in Saskatchewan] on Kodak stock and

[Wooden Shoes] on Pathé stock. Note the difference in both perforation shape and pattern.....36 


\section{Introduction}

My research in the private specialized media section at Library and Archives Canada (LAC) is focused on the large collection of $28 \mathrm{~mm}$ films and their significance to Canadian film history prior to 1939. Extant Canadian films from this period are predominantly nontheatrical and government produced, centered on utilitarian ideas of nationalism, education, and economic development. Prior to the formation of government-funded film production in 1917, many large Canadian companies such as Massey-Harris and Canadian Pacific Railway funded their own films for promotional use. These films were not intended to be screened for Canadian citizens, but to international audiences in hopes of encouraging immigration and commerce. This innovative use of the new medium and the government's motivation to disseminate Canadian content to Canadian audiences led to the establishment of two fundamental film producers in Canada: the provincial Ontario Motion Picture Bureau (OMPB) in 1917 and the federal Exhibits and Publicity Bureau (E\&PB) in 1918. From this point forward, both of these bureaux began producing films on Canadian topics to be shared with both Canadians and global audiences. Both bureaux made the choice to distribute their films on the unique and short lived Pathé-Frères $28 \mathrm{~mm}$ format.

The Pathé-Frères 28mm format, also known as Pathéscope, was introduced in 1912 along with the Pathé-KOK projector. The Pathéscope format was instrumental in the developing the nontheatrical film market, meaning films meant for exhibition in venues outside of a traditional movie theatre. The Pathé $28 \mathrm{~mm}$ formats popularity was largely due to it being the first successful safety stock, eliminating the hazard presented by nitrate motion picture stock. Until 1951 most film used cellulose nitrate as the thin flexible base that holds the emulsion and forms the structure. Cellulose nitrate was extremely flammable and fires like the 1909 Pittsburgh film 
exchange that killed ten people pushed the film industry to develop a non-flammable alternative. ${ }^{1}$ These films, often called "safety stock" used cellulose acetate for the base. Initially this stock was used for small gauge formats intended for home viewing or to be used by amateur filmmakers. Safety stock was commercially introduced first by Pathé with Pathéscope $28 \mathrm{~mm}$ in 1912, but Kodak's introduction of 16mm safety stock in 1923 quickly took hold. After 1951, all motion picture stock for theatrical or nontheatrical exhibition was safety.

LAC's unique collection of $28 \mathrm{~mm}$ films from this period has significant importance to Canadian film history, yet because of the limitations of the audiovisual database search functions, it is relatively inaccessible, and its inaccessibility has resulted in its being under researched. This paper and accompanying appendix aim to provide researchers with a tool for greater access to this unique collection. LAC promotes these unique $28 \mathrm{~mm}$ films as one complete collection, yet it is not one collection but grouping of films spread across 34 separate fonds. By creating a comprehensive list of the over $1,00028 \mathrm{~mm}$ films at LAC, and by providing the required contextual and historical background, this document will enable researchers to better understand this collection and the diverse history it reflects.

LAC first acquired its $28 \mathrm{~mm}$ film collection in 1971 and it continued to acquire $28 \mathrm{~mm}$ titles from around the world until 2008. Today, the $28 \mathrm{~mm}$ collection is one of the largest in the world, comprising 1,135 titles across 34 fonds. The LAC preservation activities website page states that "[ $\mathrm{t}]$ his $28 \mathrm{~mm}$ film collection provides the most significant record of governmentsponsored film production in Canada before the creation of the National Film Board in 1939."2 While LAC thus publicly recognizes the historical significance of this collection, it does not

\footnotetext{
${ }^{1}$ David Pierce, “The Legion of the Condemned- Why American Silent Films Perished," Film History 9, no. 1 (1997), 11.

2 “Preservation Activities," Library and Archives Canada, accessed May 12, 2019, http://www.baclac.gc.ca/eng/about-us/preservation/Pages/preservation-activities.aspx\#g
} 
provide a website link to guide researchers to the titles of the films in the collection, and the public-facing audiovisual database does not recognize film gauge search terms (Fig. 1). Basic search entries for keywords such as " $28 \mathrm{~mm}$ " as well as advanced searches that include a fonds accession number yield only a few results out of possible hundreds. LAC's database does not

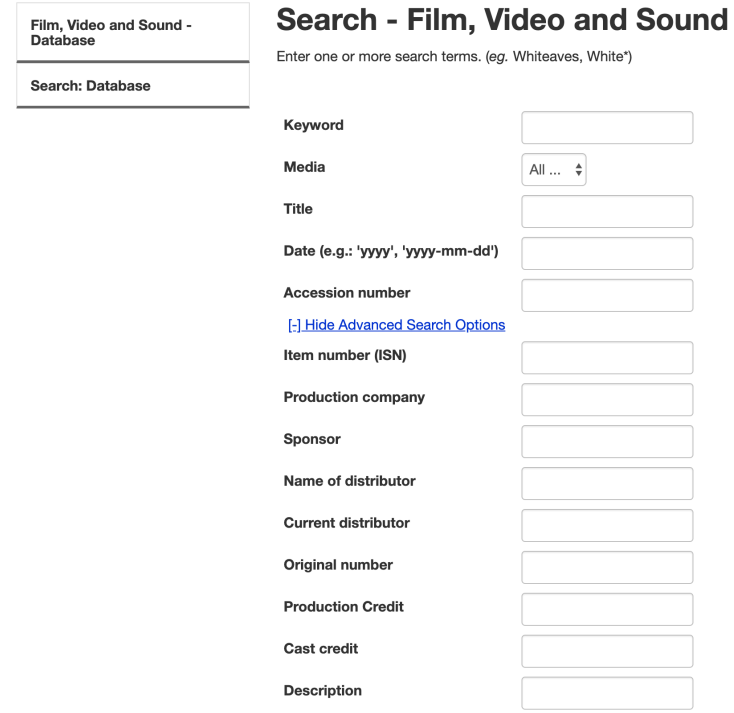

Figure 1. Screenshot of LAC Film, Video and Sound search function. From LAC website. http://www.bac-lac.gc.ca/eng/discover/films-videos-sound-recordings/film-video-sounddatabase/Pages/search.aspx allow researchers to search by physical properties and therefore, without knowing specific titles, fonds, or accession numbers, accessing the films is nearly impossible. The database's technological inability to recognize keywords relating to film gauge and the vague web

access that LAC provides are at the core of why this collection remains under researched. While providing online access to a complete database of $28 \mathrm{~mm}$ films at LAC is beyond the scope of this project, this spreadsheet and the interesting films that it has uncovered suggest future paths for subsequent research.

The thesis begins with a review of the current literature pertaining to early Canadian film production, Pathé $28 \mathrm{~mm}$ and nontheatrical film, and the issues faced by Canadian audiovisual film archives. What follows is a discussion of the central histories required to best establish an understanding of this collection. It begins with an analysis of Pathé $28 \mathrm{~mm}$ and is pivotal role in nontheatrical film exhibition followed by a short history of Canadian cinema and the emergence of 28mm film between 1912 and 1917, followed by a brief analysis of Pathé and its pivotal role 
in nontheatrical film exhibition. Finally, I will give an overview of the custodial history of LAC's $28 \mathrm{~mm}$ collection and its significance within the broader sphere of Canadian audiovisual preservation. The remainder of this thesis will be spent discussing the formation of the physical database, the methodology that established it and some interesting films that I have identified so far. Together, this thesis serves as the most effective guide to ways of accessing this collection, and my hope is that it will generate further research into the films and histories it contains. 


\section{Literature Review}

The purpose of this review is to address the existing literature on Canadian cinema prior to 1939 , and the manufacturer of $28 \mathrm{~mm}$ film, Pathé-Frères. This survey situates this film collection within the broader history of Canadian cinema and reveals the scarcity of research material that focuses on nontheatrical films production in Canada between 1917 and 1930 and the $28 \mathrm{~mm}$ film format.

\section{Canadian Film History before 1939}

Film scholar Peter Morris published Embattled Shadows: A History of Canadian Cinema 1895-1939 in 1978 and over forty years later, it remains the most significant contribution to research into early Canadian film history. ${ }^{3}$ Morris chronicles the production, distribution and exhibition of film in Canada before the establishment of the National Film Board (NFB) in 1939. Chapter 5, "The State and the Movies," focuses on the foundation of the OMPB, the E\&PB, and Pathéscope of Canada, the production companies whose films form the bulk of the $28 \mathrm{~mm}$ collection held at LAC. Pathéscope of Canada was a film exchange that had ties to Pathéscope USA and Pathé-Frères in France. In this chapter Morris argues that the primary focus of the federally funded $\mathrm{E} \& \mathrm{~PB}$ and the provincially funded OMPB was on educational films geared towards farmers, school children, and films that promoted European immigration to Canada. Due to the focus on nontheatrical educational films, both bureaux chose to distribute their films on the only available non-flammable safety stock of the period, Pathé $28 \mathrm{~mm}$.

\footnotetext{
${ }^{3}$ Morris, Embattled Shadows: A History of Canadian Cinema 1895-1939 (Montreal: McGill-Queen's University Press, 1978).
} 
Morris's work expands on Charles F. Backhouse's 1974 book, Canadian Government Motion Picture Bureau 1917-1941. ${ }^{4}$ Backhouse's book is an overview of the Canadian Government Motion Picture Bureau from its creation as E\&PB to its end. He divides the history of the CGMPB into four distinct periods beginning with its formation in 1918, its strong active years between 1920-1931, the decline between 1931-1939, and finally its being folded into the NFB after 1939. Using primary sources from Public Archives of Canada (PAC), Backhouse shows that initially the E\&PB was hesitant to use the Pathé $28 \mathrm{~mm}$ gauge due to it not being the professional industrial standard 35mm. However, the E\&PB had two modes of global distribution, theatrical meaning exhibited in a theatre, and nontheatrical being venues that are not designed as a traditional theatre, the latter of which used the Pathe $28 \mathrm{mnm}$ format. ${ }^{5}$ The increasing financial costs of the E\&PB were a contributing factor to its eventual closure, and a significant amount of money was spent in the late 1920s on updating lab equipment from the outdated $28 \mathrm{~mm}$ nontheatrical format to $16 \mathrm{~mm}^{6}$

Gerald Graham, Former Director of Technical Operations and Research at the NFB, wrote Canadian Film Technology: 1896-1986 in 1989. ${ }^{7}$ It traces the development of the Canadian film industry by looking at the impact film technology had on Canadian productions. Using film's technological evolution as a guide, Graham's book unearths the often-overlooked differences between Canada's developing film industry and that of the United States or Europe. The film industry in Europe and the United States evolved at a time when those countries were already relatively developed both economically and socially; they saw film as a profitable means

\footnotetext{
${ }^{4}$ Charles F. Backhouse, Canadian Government Motion Picture Bureau 1917-1941, (Ottawa: Canadian Film Institute, 1974).

${ }^{5}$ Ibid., 11.

${ }^{6}$ Ibid., 18.

${ }^{7}$ Gerald Graham, Canadian Film Technology: 1896-1986 (London: Associated University Press, 1989).
} 
of expanding their existing entertainment industry. At the same time, Canada was still developing as a nation in terms of its agriculture and resources, the need for film in Canada was, "primarily a means of educational and promotional tool to explain our vast and fragmented country to ourselves and to encourage settlers from other lands to come to Canada and fill out the vast emptiness." ${ }^{1}$ Cities in Europe and the United States had large urban centres that had welldeveloped entertainment industries prior to the introduction of motion pictures in 1896 and establishing film as a means of entertainment came naturally. ${ }^{9}$ Graham argues that with the end of the First World War, both provincial and federal governments in Canada began to pay more attention to the use of nontheatrical film for informational and instructional purposes. ${ }^{10}$

Canadian film historian Gerald Pratley wrote Torn Sprockets: The Uncertain Projection of the Canadian Film, in $1987^{11}$. The majority of Pratley's book is focused on the history of Canadian film after the NFB was formed in 1939. While not as detailed as Morris's work, Pratley summarizes Canadian film production before the formation of the NFB. For Pratley, the early years of Canadian film, from 1886-1939, is a period of, "interesting, determined, usually well-intentioned...with little impact on audiences and containing little value for present-day study." ${ }^{2}$ This was a period that produced nothing of recognition in terms of stars, directors, or pictures, in comparison to what was happening in Hollywood. ${ }^{13}$ During this period, many theatres in Canada were owned by Americans who saw little value in investing in exhibiting Canada films when Hollywood had the stars and stories that captivated audiences globally. ${ }^{14}$ Similar to Morris, Pratley chronicles a history of early Canadian films beginning with industrial

\footnotetext{
${ }^{8}$ Ibid., 22.

${ }^{9}$ Ibid., 56.

${ }^{10}$ Ibid., 57.

${ }^{11}$ Gerald Pratley, Torn Sprockets: The Uncertain Projection of the Canadian Film, (Toronto: Associated University Presses, 1987)

${ }^{12}$ Ibid., 13.

${ }^{13}$ Ibid., 13.

${ }^{14}$ Ibid., 14.
} 
companies by non-Canadians for non-Canadian audiences. Chapter 4, "The Beginning of Canadian Documentaries," explores primarily the formation the E\&PB with only a brief mention of the OMPB.

Film archivist Rosemary Bergeron's 2007, “A History of the Newsreel in Canada: A Struggle for Screen Time," ${ }^{15}$ traces the history of Canadian newsreel productions and explores why Canadian-produced newsreels struggled to reach Canadian audiences who were predominantly seeing American produce newsreels in Canadian theatres. Bergeron begins by addressing how the Canadian public's desire for a Canadian-focused newsreel came during the First World War when American films focused either on neutrality or, when America entered the conflict, American issues. ${ }^{16}$ Canada's involvement in the war effort brought an increase in Canadian Nationalism. The increased sense of nationalism caused Canadian audiences to wonder why they were seeing American focused newsreels when America had yet to join the effort. The Canadian War Records Office began supervising British cameramen filming Canadian troops to insure they were capturing the Canadian effort in a way that was favorable for the Canadian public. This increased demand for Canadian content in newsreels suggested to the post-World War I Canadian Government that films had a potential to foster a strong sense of Canadian Nationalism. It suggested that films could be made to educate citizens about culture, geography, and industrial potential. ${ }^{17}$ The demand for Canadian-focused newsreels sparked the Department of Public Information to contract Pathéscope of Canada in 1919 to ask it to produce The Canadian National Pictorial. The Canadian National Pictorial covered stories that promoted industry, significant or official events, or major attractions within Canada. It also featured a

\footnotetext{
${ }^{15}$ Rosemary Bergeron, "A History of the Newsreel in Canada: A Struggle for Screen Time," The Movie Image 7, no. 2 (Fall 2007): 25-54, https://muse-jhu-edu.ezproxy.lib.ryerson.ca/article/235600/pdf

${ }^{16}$ Ibid., 27.

${ }^{17}$ Ibid.
} 
"made in Canada," segment that featured the manufacturing of Canadian goods. ${ }^{18}$ When the Department of Public Information was closed in 1919, the production of The Canadian National Pictorial was transferred to the E\&PB. Near the end of 1921, the E\&PB withdrew the Pathéscope of Canada funding for the production of The Canadian National Pictorial and Canadian newsreel soon ceased production. ${ }^{19}$

\section{Pathé 28mm and nontheatrical film}

Film archivist Anke Mebold and film scholar Charles Tepperman published, "Resurrecting the Lost History of $28 \mathrm{~mm}$ film in North America," ${ }^{20}$ in 2003. It traces the short history of $28 \mathrm{~mm}$ film in North America, identifying it as a brief but popular alternative to the $35 \mathrm{~mm}$ cellulose nitrate industry standard. Mebold and Tepperman discuss the success Pathé $28 \mathrm{~mm}$ achieved in the United States and Canada, displaying to film manufactures there was a market for films intended to be screened outside of a theatre (nontheatrical). ${ }^{21}$ They begin by pointing out that the introduction of $28 \mathrm{~mm}$ film in 1912 came right in the middle of the transitional period of cinema, when the medium expanded from a novelty or attraction to a mode of production and a major industry. ${ }^{22}$ They show that as it was an expensive luxury at the time, the Pathé KOK projector and $28 \mathrm{~mm}$ film equipment were marketed to wealthy families. Finally, Mebold and Tepperman look at the role $28 \mathrm{~mm}$ played in educational and industrial films in Canada such as those produced by the OMPB and the University of Alberta that had invested heavily in the format. Mebold and Tepperman conclude by suggesting that $28 \mathrm{~mm}$ flourished in during a unique moment in cinema history when ideas of what movies could be used for were in

\footnotetext{
${ }^{18}$ Ibid., 28.

${ }^{19}$ Ibid., 31 .

${ }^{20}$ Anke Mebold, Charles Tepperman, "Resurrecting the lost history of $28 \mathrm{~mm}$ film in North America," Film History 15, no. 2 (January 2003): 137-151.

${ }^{21}$ Ibid., 137.

${ }^{22}$ Ibid.
} 
their earliest stages of development; they see that ultimately the $28 \mathrm{~mm}$ format showed there was a viable market for a nontheatrical film format. ${ }^{23}$

In 1993, film historian Richard Abel published, "In the Belly of the Beast: The Early Years of Pathé-Frères," 24 focusing on the development of Pathé as a company from its founding by Charles Pathé in 1896 to just before the First World War. Abel argues that during this period Pathé underwent rapid growth that influenced film companies in France and internationally. ${ }^{25}$ This international influence shaped the national film industry of countries as far as Russia and the United States. Abel does not mention Canada, and I would suggest Pathé had a large impact on the development of the Canadian national film industry. The beginning of Abel's article traces the history of Pathé in France, defining two distinct expansion periods for the company. The first expansion of the company increased its production facilities to meet the growing demand for film and equipment, in part because Charles Pathé targeted rural fairgrounds as places to exhibit Pathé productions. ${ }^{26}$ The second expansion phase came when Pathé began opening theatres and distributing its films in a weekly rental program for exhibitors. ${ }^{27}$ Abel suggests that one of Pathé main business principles was to exploit new technology for commercial benefits. ${ }^{28}$ This business model is important in contextualizing why Pathé adopted the nontheatrical $28 \mathrm{~mm}$ format, first marketed in 1912 along with a selection of films from Pathé's library. The Pathé KOK projector and $28 \mathrm{~mm}$ film gauge were new technologies that opened a market that virtually no other company was competing for. The popularity of the $28 \mathrm{~mm}$

\footnotetext{
${ }^{23}$ Ibid., 149.

${ }^{24}$ Richard Abel, "In the Belly of the Beast: The Early Years of Pathé-Frères," Film History 5, no. 4 (December 1993): 363-385, https://archivaria.ca/index.php/archivaria/article/view/13603

${ }^{25}$ Ibid., 363.

${ }^{26}$ Ibid., 366.

${ }^{27}$ Ibid., 369.

${ }^{28}$ Ibid., 364.
} 
gauge lead to Pathé further developing nontheatrical gauges such as $9.5 \mathrm{~mm}$ and ultimately started the market for nontheatrical cinema.

Trade journals dedicated to amateur filmmaking provide a wealth of information on early nontheatrical film equipment and the history of $28 \mathrm{~mm}$ film in North America. While these journals are focused on the use of amateur film equipment by the public, the history of the technology outlined within them gives insight into success and shortcomings of the same equipment being used by the Canadian film bureaux. Published in 1923, a Transactions of the Society of Motion Picture Engineers article titled, "The Beacon Portable Motion Picture Projector," 29 argues that the portable motion picture projector will be useful in expanding what was currently thought of as the motion picture industry. ${ }^{30}$ The author reviewing the new Beacon projector makes note of some various situations where a portable projector would be of value over a professional projector before noting that the Beacon projector is available for either $35 \mathrm{~mm}$ or $28 \mathrm{~mm}$ film stock. ${ }^{31}$ A 1930 article in Cinematographic Annual titled, "Cinemachinery for the Personal Movie,"32 outlines the relatively young ( by that point,) history of amateur film equipment. The author notes that the Pathéscope $28 \mathrm{~mm}$ was the, "first projector designed exclusively for home use," but suggests that regardless of Pathé manufacturing 28mm equipment for close to 20 years by the time the article was written, it was not until $16 \mathrm{~mm}$ was introduced that manufacturers realized the, "distinct needs and possibilities of the amateur field." 33 In the same year, Merritt Crawford wrote an article in the amateur cinema journal Movie Makers titled,

\footnotetext{
${ }^{29}$ J.R. Mitchell, “The Beacon Portable Motion Picture Projector,” Transactions of the Society of Motion Picture Engineers 16, (May 1923): 225-232, https://muse-jhu-edu.ezproxy.lib.ryerson.ca/article/235600/pdf

${ }^{30}$ Ibid., 225.

${ }^{31}$ Ibid., 226-227.

32 “Cinemachinery for the Personal Movie,” Cinematographic Annual 1, (1930): 513-539, https://muse-jhuedu.ezproxy.lib.ryerson.ca/article/235600/pdf

${ }^{33}$ Ibid., 527.
} 
"Movie Makers The first thirty years," 34 that is perhaps the first attempt to chronical the history of amateur film gauges and equipment. Crawford argues that the first 20 years of nontheatrical film equipment was "chaotic," as each "new device established standards of its own without relation to any other and with no particular regard for the need of the field as a whole." ${ }^{\prime 3}$ This lead to a slew of unusual and short lived gauges, mediums, and devices, all which lead to the introduction of $16 \mathrm{~mm}$ film in 1923. In regard to Pathéscope $28 \mathrm{~mm}$, Crawford points out it was the first, "nonflam" film commercially available, along with that, Pathéscope offered a film rental program and later a camera. ${ }^{36}$ Crawford also suggests that part of the short-lived success of Pathéscope could have come from the fact that companies other than Pathé, such as Victor and the Beacon projector, adopted the format and sold products compatible with the Pathé stock.

\section{Canadian Audiovisual Archives}

LAC archivist Sarah Cook’s 2017, “Shrouded History: The Canadian Film and Photo Unit, Records Creation, Reuse, and the Recontextualizing of "Lost" Audiovisual Heritage,"37 looks at the complex history of the Canadian Film and Photography Unit's (CFPU) records after World War II and the difficulties of reflecting this history through the current LAC database. Cook traces both the history of the unit's records creation, including the AV materials and textual records, as well as the history of how the unit's recorders were managed after the war. By doing so, Cook demonstrates the issues of current archival descriptions at LAC that do not reflect the complex histories of AV records. ${ }^{38}$ Cook notes that the LAC Film, Video, and Sound

\footnotetext{
${ }^{34}$ Merritt Crawford, "Movie Makers The first thirty years," Movie Makers (December 1930): 755-785, https://musejhu-edu.ezproxy.lib.ryerson.ca/article/235600/pdf

${ }_{35}^{35}$ Ibid., 755.

${ }^{36}$ Ibid., 784.

${ }^{37}$ Sarah Cook, "Shrouded History: The Canadian Film and Photo Unit, Records Creation, Reuse, and the Recontextualizing of "Lost" Audiovisual Heritage," Archivaria 83 (Spring, 2017): 125-148, https://archivaria.ca/index.php/archivaria/article/view/13603

${ }^{38}$ Ibid., 128.
} 
database describes AV material at an item level, and searches across various fonds and collections to pull results. However, the AV database then separates the items from their fonds, thus losing the custodial history of the collection. ${ }^{39}$ The fonds is searchable in the archives database, but do not show the individual item level. Cook concludes by suggesting that to properly understand and provide access to the CFPU AV materials, there needs to be a way to show the full history of these large collections that have been transferred to numerous institutions before coming to LAC.

${ }^{39}$ Ibid., 147. 


\section{Chapter 1: The Rise of Nontheatrical Cinema on 28mm film}

The distinction between amateur and professional films and equipment in the early years of cinema (before 1910) is somewhat hard to define. Film historian Deac Rossell notes, "[i]n the early years, there were many varied ideas about how to use moving pictures and what marketplace would secure their future; consequently, most apparatuses retrospectively called "amateur" were designed for film narrower than the $35 \mathrm{~mm}$ bands that later became the standard size. ${ }^{\not 0}$ Interest in nontheatrical film apparatuses occurred almost instantaneously alongside Edison and the Lumiere's initial public exhibitions their inventions. In 1899, two amateur film gauges were introduced, $17.5 \mathrm{~mm}$, created by Brit Acres and $13 \mathrm{~mm}$ created J.A Preswich. ${ }^{41} \mathrm{In}$ Europe, devices were designed to attach to the front of magic lantern projectors, turning them into a 'crude projector' marketed in 1896. In the USA, American Parlor Kinetoscope Company, offered a smaller version of the Edison Kinetoscope that used transparent paper instead of film. ${ }^{42}$

The Ikonograph projector, produced by the Ikonograph Commercial Company of Manhattan, introduced a projector around 1904 that resembled what was being used as a professional projector at the time. ${ }^{43}$ The Ikonograph projector used $17.5 \mathrm{~mm}$ film, which was created by splitting the professional standard of $35 \mathrm{~mm}$ cellulose nitrate in half. The Ikonograph projector system was short lived, and not long after its introduction the company was bankrupt. Articles in amateur movie trade journals mentioned earlier support Rossell's statement that the early years of nontheatrical cinema was a frenzy of odd formats and machines that never became standardized or widely popular until the introduction of Kodak's $16 \mathrm{~mm}$ film gauge in 1923 . The

\footnotetext{
${ }^{40}$ Deac Rossell, “Amateur Film,” in Encyclopedia of Early Cinema, ed. Richard Abel (London: Routledge, 2005), 18, https://muse-jhu-edu.ezproxy.lib.ryerson.ca/article/235600/pdf

${ }^{41}$ Ibid., 18.

${ }^{42}$ Ben Singer, "Early Home Cinema and the Edison Home Projecting Kinetoscope," Film History 2, no. 1. (Winter 1988): 38, http://www.jstor.org/stable/3814949

${ }^{43}$ Ibid., 40.
} 
explosion of companies selling equipment for $16 \mathrm{~mm}$ film present in trade journals such as Movie Makers, suggest that the introduction of $16 \mathrm{~mm}$ film truly launched a market for nontheatrical amateur film. However, prior to the widespread and long-lasting popularity of Kodak's $16 \mathrm{~mm}$, there was Pathé-Frères 1912 Pathé KOK home projector along with a selection $28 \mathrm{~mm}$ Pathéscope cellulose diacetate films. ${ }^{44}$ Cellulose diacetate was a predecessor to the more commonly used cellulose acetate base, a non-flammable alternative to nitrate.

When Pathé initially introduced their Pathé KOK projector it was not as an affordable novelty device. As noted by Mebold and Tepperman, “[t]he Pathé KOK was not marketed as a democratic or affordable projection system available to the majority of consumers." 45 The $28 \mathrm{~mm}$ film gauge that Pathé settled on was different from the later and more dominant gauges of nontheatrical amateur film, (Pathé-Baby $9.5 \mathrm{~mm}, 8 \mathrm{~mm}$, Super $8 \mathrm{~mm}$, and 16mm). Pathé 28mm film stock was big, only $7 \mathrm{mms}$ shy of the professional standard $35 \mathrm{~mm}$ which became adopted as a professional standard in $1909 .{ }^{46}$ This meant that images were larger and less grainy than the smaller gauges. ${ }^{47}$ Film scholar Ben Singer suggests that, "[ $\left.\mathrm{t}\right]$ his larger substandard size did, however, enable a bigger picture to be projected on the screen before graininess became a problem, and thus Pathé may have chosen it in a deliberate attempt to make the Pathéscope projector and $28 \mathrm{~mm}$ gauge attractive to schools, churches, and clubs, where a large picture would be advantageous." 48 28mm Pathéscope films featured a unique perforation pattern, three perforations one side of the image but only one on the other side. The advantage to this was it was nearly impossible to thread the film improperly. Equally important, “...the unique

\footnotetext{
${ }^{44}$ Abel, "In the Belly of the Beast," 337.

${ }^{45}$ Mebold, Tepperman, "Resurrecting the Lost," 140.

${ }^{46}$ Paolo Cherchi Usai, Silent Cinema: An Introduction (London: BFI Publishing, 2000), 2.

47 Singer, "Early Home Cinema,"44.

${ }^{48}$ Ibid., 44.
} 
perforation configuration, protected by a Pathé patent, insured that Pathé $28 \mathrm{~mm}$ films could only be shown on the Pathéscope"49

Appearing in a 1919 issue of American journal, Arts and Decoration, the ad for the latest Pathé $28 \mathrm{~mm}$ KOK projector called, "the new premier," emphasised its use in venues other than homes. The bold writing at the bottom of the ad claimed, "[c]lubs, churches and schools, find in the New Premier Pathéscope just what they need to entertain interest and instruct." 50 The ad goes on to state that the Pathéscope Exchange has over 1,100 titles that suit a wide variety of interests. Perhaps most interesting, this 1919 ad advertises both the projector, the Pathéscope film rental library, and the Pathéscope camera, a $28 \mathrm{~mm}$ camera for personal use. ${ }^{51}$ However, despite the ad emphasizing the use of the Pathé KOK projector for venues outside of the home, Mebold and Tepperman point out that when Pathé was introduced in the UK and America under the name Pathéscope, it was primarily marketed as a device for the private use of the wealthy. ${ }^{52}$

Both Pathé-Frères in France and Pathéscope UK, USA, and Canada, offered catalogues of films to users. Pathéscope UK, USA, and Canada had links back to Pathé-Frères and were set up as exchanges distributing Pathéscope prints. Stores or libraries would get films from these exchanges where Pathé films ranging in a variety of subjects could be rented or even purchased. Many early films available were reduction prints of Pathé productions; however, by around 1915, catalogues in the USA began to display American-made features that were available for rent. ${ }^{53}$ Pathéscope had an extensive library of films and a subscription service that varied for an annual fee of between fifty and one hundred dollars. The films could be rented from one night to

\footnotetext{
49 Ibid., 44.

50 “The New Primer Pathéscope," Arts and Decoration, September, 1919, 248, https://archive.org/details/artsdecoration11 newy/page/248

${ }^{51}$ Ibid., 248.

52 Mebod, Tepperman, "Resurrecting the Lost," 141.

53 Ibid., 142.
} 
two weeks, depending on the subscription plan of the user. ${ }^{54}$ Pathéscope advertised their machines as the only ones officially recognized by the National Education Association, making Pathé KOK projectors, at least for a few years, the only projector and film company recognized safe for school use. ${ }^{55}$ While Pathéscope USA continued to target both private consumers and educational audiences throughout the 1910s, Pathéscope of Canada found the non-flammable $28 \mathrm{~mm}$ film stock and its ability to be screened in nontheatrical settings for educational purposes the more useful. The two Canadian government film bureaux supported this view.

${ }^{54}$ Ibid., 143.

55 Ibid., 145. 


\section{Chapter 2: Early Canadian film and the Introduction of 28mm film (1912- 1917)}

The history of Canadian cinema began long before John Grierson established the NFB in 1939. Dating back to the earliest parts of the twentieth century, some of Canada's first industrial corporations like Massey-Harris and Canadian Pacific Railway (CPR) recognized film's potential as a promotional tool. This realization in combination with the lack of Canadian film production companies required that they outsource the production to international companies. For example, in 1898, Massey-Harris hired the Edison Company to produce a series of films promoting its new agricultural equipment. These films, made on the currently available cellulose nitrate film stock, were shown in major cities throughout Canada before finally being distributed in the United Kingdom in hopes of boosting international sales. ${ }^{56}$ In 1902 , CPR hired a group of British cameraman and technicians to travel and film the Canadian way of life to be distributed and marketed in the United Kingdom through the Charles Urban Trading Company. ${ }^{57}$ By bringing Canada to the United Kingdom, these films were meant to encourage immigration and boost economic development.

Simultaneously, early American fiction films were booming in Canada, making it increasingly more difficult for Canadian-produced films to compete with the large and fastgrowing industry. Canadian film historian Gerald Pratley points out that during this period a large number of those in charge of Canadian film distribution and exhibition were Americans who had moved to Canada and, "there is little evidence available to suggest that those involved...considered it in any way essential that the films they showed should include domestic

\footnotetext{
${ }^{56}$ Peter Morris, Embattled Shadow: A History of Canadian Cinema 1895-1939 (Montreal: McGill-Queen's University Press, 1978), 32.

${ }^{57}$ Ibid., 33.
} 
productions. ${ }^{58}$ Some notable exceptions include early Canadian features such as Evangeline (1913) and Back to God's Country (1919). But even for a film like Evangeline, the first Canadian feature-length film about the expulsion of the Acadians, the notion that it would spark a sense of national pride amongst Canadians was far reaching. Only one newspaper in Montreal made note of its release and production in the country. ${ }^{59}$ It was not until the early days of the First World War that Canadian audiences began to question why they were not seeing Canadian newsreels about Canadian troops during the war. Having seen the popular demand by Canadian citizens for films that depicted uniquely Canadian themes, both the provincial government of Ontario and the Federal government decided to begin funding government sponsored film production. The films were not fiction films intended to compete against the dominant American market, but educational, instructional and travel focused.

As mentioned above, much of the successful sponsored film output during the period before the formation of either of the bureaux was made in the interests of promoting Canadian industry or encouraging European immigration. ${ }^{60}$ The OMPB was founded May of 1917 and S.C. Johnson, who worked for the provincial Department of Agriculture, was appointed its first director. Johnson believed that motion pictures possessed a stronger ability for education than literature. He had written several books on farming and believed that motion pictures could be used to educate farmers who had no formal education. ${ }^{61}$ Established as part of the provincial treasurer's department, the OMPB's purpose was to create and distribute, "educational work for farmers, school children, factory workers, and other classes. ${ }^{" 62}$ Despite its formation in 1917, the

\footnotetext{
${ }^{58}$ Paratley, "Torn Sprockets," 23.

${ }^{59}$ Zoë Constantinides, "The Myth of Evangeline and the Origin of Canadian National Cinema," Film History 26, no. 1 (2014): 52, https://muse-jhu-edu.ezproxy.lib.ryerson.ca/article/542047/pdf

${ }^{60}$ Morris, Embattled Shadows, 129.

${ }^{61}$ Ibid., 137.

${ }^{62}$ Ibid., 138.
} 
OMPB did not officially begin producing its own films until 1923. In the interim, as described below, production was contracted out to companies in Toronto while the OMPB took care of the content and distribution. ${ }^{63}$

On 19 September 1918, shortly after the formation of the OMPB, the federal government's E\&PB was founded. The E\&PB was formed out of the Trade and Commerce department and therefore films needed to demonstrate a significant amount of Canadian goods or industrial advertising. ${ }^{64}$ The E\&PB became the first official federal government film production unit established in the world. ${ }^{65}$ Under the direction of Ben Norrish, its first director, the bureau began ordering equipment and making recommendations that all publicity films produced by any department of the government be passed through the E\&PB. ${ }^{66}$ Shortly after its formation, the E\&PB began distributing films in Europe which was important because, "countries of Europe, just recovering from a war, were eager to purchase raw materials and other trade goods. ${ }^{977} \mathrm{In}$ April of 1923, the E\&PB officially changed its name to the Canadian Government Motion Picture Bureau and began producing the Seeing Canada series. This series, each being one reel or approximately ten minutes, was initially designed to be used as a promotional tool abroad for immigration to Canada. However, following the post WWI rise of Canadian nationalism, the film series became widely popular within Canada, providing many Canadians with the first images they had seen of Canada in a movie theatres, churches or schools. ${ }^{68}$ As mentioned earlier, distribution of CGMPB films was either theatrical on $35 \mathrm{~mm}$ nitrate, or nontheatrical, on $28 \mathrm{~mm}$ Pathé safety stock. After the name change, the CGMPB expanded its global distribution to more

\footnotetext{
${ }^{63}$ Ibid., 137.

${ }^{64}$ Backhouse, Canadian Government, 6.

${ }^{65}$ Ibid., 133.

${ }^{66}$ Backhouse, Canadian Government Motion Picture Bureau, 6.

${ }^{67}$ Ibid., 8.

${ }^{68}$ Morris, Embattled Shadows, 135.
} 
countries in continental Europe, Japan, China, and countries in South America. ${ }^{69}$ In the USA, Bray Productions Incorporated whose headquarters were in New York handled the distribution of CGMPB films, many of which were well received. ${ }^{70}$

Both OMPB and E\&PB became key to the wide spread use of Pathé $28 \mathrm{~mm}$ in Canada. Both forged important relations to Pathéscope of Canada. Established in 1914 by H. Norton DeWitt, Pathéscope of Canada had initially focused on the selling of $28 \mathrm{~mm}$ projectors and the distribution of 28mm Pathéscope films in Canada from the catalogues of Pathéscope USA and Pathé-Frères in France. ${ }^{71}$ Because the focus of the OMPB and E\&PB on educational films, the professional industry standard $35 \mathrm{~mm}$ nitrate film stock posed a major problem for nontheatrical exhibition. The solution to this problem came with the Pathé $28 \mathrm{~mm}$ diacetate non-inflammable safety stock and the portable easy to use Pathé KOK projectors. As mentioned earlier, the OMPB did not begin producing its own films until 1923 and prior to this, the production of films was handled by other companies. The OMPB had a relationship with Pathéscope of Canada who began to focus on producing films for OMPB beginning around $1918 .{ }^{72}$ Although Pathéscope of Canada was producing films, as Morris notes, "this was to be production sponsored and paid for by others. Pathéscope never risked its own capital to produce for its own account." ${ }^{.73}$ The production activities of Pathéscope of Canada between the years 1917 to 1923 proved to be extremely lucrative for the company which, in 1918, opened a laboratory located on King Street, Toronto. ${ }^{74}$ However, in 1923 the OMPB opened its own production studies in Trenton Ontario, therefore cutting a large portion of Pathéscope of Canada's income.

\footnotetext{
${ }^{69}$ Backhouse, Canadian Government Motion Picture Bureau, 12.

${ }^{70}$ Ibid., 12.

${ }^{71}$ Ibid., 138.

${ }^{72}$ Ibid., 138-139.

${ }^{73}$ Ibid., 138.

${ }^{74}$ Ibid., 141.
} 
Although much of Pathéscope of Canada's production output was for the OMPB and E\&PB, other Canadian establishments took note of the $28 \mathrm{~mm}$ safety stock and used it for their own productions or collections. The University of Alberta began to amass their own $28 \mathrm{~mm}$ film collection called the "Winged-Foot Travelling Library of Moving Picture Films and Lantern Slides." ${ }^{75}$ Comprised of both educational and reduction prints of theatrically released films, the University of Alberta collection was formed with the intent of showing these films to remote areas of the province. Unlike the OMPB or E\&PB, the University of Alberta was not producing or sponsoring the production of films but building a travelling collection of films made by the bureaux in Canada or theatrical release films reduced down to the $28 \mathrm{~mm}$ format. Filmcraft Industries, formed in 1919 by Ontario-born Blaine Irish, would become a large producer of both $28 \mathrm{~mm}$ sponsored and some theatrical films. ${ }^{76}$ Filmcraft was large enough to have its own laboratory located in Toronto with tinting and toning capabilities and became a tough competitor for Pathéscope of Canada. ${ }^{77}$ The Saskatchewan and Nova Scotia governments instead, like the OMPB and the E\&PB, hired Pathéscope of Canada to produce their films. Eventually, Pathéscope of Canada cameraman Richard "Dick" Bird would start his own production company in Saskatchewan. ${ }^{78}$ In the current LAC collection a few films were produced by a company called Regina Films Limited, however it is unclear if that was Dick Bird's company or not.

The use of the $28 \mathrm{~mm}$ Pathéscope gauge allowed both the bureaux and the smaller provincial production companies to distribute their films to areas that did not have theatres, or to be used as an educational tool in schools. But the choice to use the Pathé $28 \mathrm{~mm}$ gauge proved to be a mistake, at least for the OMPB. By 1925, Kodak's 16mm film had been out for a few years

\footnotetext{
${ }^{75}$ Mebold, Tepperman, "Resurrecting the Lost," 146.

${ }^{76}$ Morris, Embattled Shadows, 144.

${ }^{77}$ Ibid., 144-45.

${ }^{78}$ Ibid., 140.
} 
and was becoming more popular as a nontheatrical format, especially with the introduction of Kodascope film exchange in 1924. While other factors were at play, one of the largest factors for the OMPB's eventual demise was, “the fact that the vast bulk of the Bureau's film collection was on the out-dated $28 \mathrm{~mm}$ and its laboratory equipment designed for $28 \mathrm{~mm}$ only was to be a major factor in the decision of the new Liberal government to close the Bureau."79 Charles Backhouse also claims that the increasing financial costs of the E\&PB was contributing factor to its eventual closure, and shows that a significant amount of money was spent in the late 1920s on updating lab equipment to the now popular $16 \mathrm{~mm}$ nontheatrical format. ${ }^{80}$ What was at one point deemed to be out-dated and eventually a contributing factor to the demise of the OMPB, the value in recent years for both the unusual $28 \mathrm{~mm}$ gauge and the content of these films was recognized.

\footnotetext{
${ }^{79}$ Ibid., 152.

${ }^{80}$ Backhouse, Canadian Government, 18.
} 


\section{Chapter 3: The 28mm Collection at Library and Archives Canada}

The custodial history of LAC's $28 \mathrm{~mm}$ collection is both broad and complex. The largest number of films are in the Graphic Consultants fonds which has the most detailed history of accession. When the OMPB dissolved in 1935, the film catalogue was split up and sold first to Associated Screen News and then finally to Father Joseph Gravelle, a priest who had purchased the films for his church. ${ }^{81}$ In 1965, the films were purchased from the church by Greg Hoy, the founder of Graphic Consultants, who recognized the historical value of their content. Hoy had founded Graphic Consultants as a stock image company in the mid 1960s and began transferring the $28 \mathrm{~mm}$ films to $16 \mathrm{~mm}$ and $35 \mathrm{~mm}$. LAC acquisition files trace the custodial history of the Graphic Consultants fonds to 1966 when Graphic Consultants reached out to the National Film Board (NFB) to inquire if the NFB would have any interest in these films. ${ }^{82}$ The correspondence from the staff of Graphic Consultants continues in the acquisition files with the Provincial Archives of Alberta, Department of Agriculture and Horticultural Societies, Canadian War Museum, and other government departments. The correspondence asks if these institutions have an interest is purchasing a copy of the $28 \mathrm{~mm}$ films or if there is interest in helping Graphic Consultants with the expensive and time-consuming transfer process.

One of the more interesting letters in the Graphic Consultants acquisition files was written 22 January 1969 to the then Secretary of State, Gerard Pelletier. The letter concerns the potential for federal government financial backing: Hoy highlights audiovisual preservation problems such as access and long-term preservation, and the increasing costs. In the letter, Hoy's main concern was to make sure these films would be preserved by transferring them to a stable

\footnotetext{
81 “Graphic Consultants Fonds,” Library and Archives Canada, accessed 25 May 2019, http://collectionscanada.gc.ca/pam_archives/index.php?fuseaction=genitem.displayItem\&rec_nbr=190826\&lang=en g\&rec_nbr_list=190826,105494,140416,98129,4238700,105872,4816192,106871,106735,106723

82 January 17,1966 . From LAC, Acquisition file, "1972-0105."
} 
medium and making them widely available to the Canadian public. Hoy also points out that a great deal of interesting footage remaining in private collections or within institutions never gets shown and expresses how he would not want that to happen to his $28 \mathrm{~mm}$ collection. ${ }^{83}$ These letters document the beginning of Canadian federal government-funded film preservation activity, and Graphic Consultants $28 \mathrm{~mm}$ collection will become one of the earliest acquisitions for the newly formed National Film Archives (NFA), part of the then Public Archives of Canada (PAC), itself one of the first protagonists in film preservation activity in Canada.

Pelletier was a key figure in setting up Canada audiovisual preservation initiatives in the late 1960s and early 1970s. On 23 July 1967, a fire had broken out in the vaults of the NFB, destroying a large part of Canada's film history from 1890-1950. ${ }^{84}$ This significant loss of Canadian film heritage rekindled the desire for a proper government-funded film preservation program. After the fire, the PAC began to collect Canadian nitrate films in order to transfer as many as possible to safety stock. Finally, in 1972, Pelletier outlined the national film policy that officially created the National Film Archives as part of the larger PAC. ${ }^{85}$

In the same year as the formation of the National Film Archives, Hoy wrote to Project Director of the new National Film Archive, William Gallaway, expressing the difficulties Graphics Consultants was having with the costly transfer process. ${ }^{86}$ He mentions that he has had several offers to sell the collection to American companies, but was willing to negotiate in order to keep the historically important collection in Canada. The acquisition records show that by early September of 1972 a deal had been made with Greg Hoy and the National Film Archive to receive the entire collection as well as the restoration equipment used for the transfer of $28 \mathrm{~mm}$ to

\footnotetext{
${ }^{83}$ Greg Hoy to Gerald Pelletier, January 22, 1969. Letter. From LAC, Acquisition files, “1972-0105”.

${ }^{84}$ Michele Wozny, "National Audiovisual Preservation Initiatives and the Independent Media Arts in Canada," Archivaria 67 (Spring 2009): 97, https://archivaria.ca/index.php/archivaria/article/view/13210/14488

85 Ibid., 99.

${ }^{86}$ Greg Hoy to William Gallaway, May 7, 1972. Letter. From LAC, Acquisition files, “1972-0105.”
} 
$35 \mathrm{~mm}$. The acquisition of the Graphic Consultants collection represented one of the first major collections acquired by the Canadian federal-government funded National Film Archive and therefore has a unique position as one the first acquisitions that began film preservation in Canada. The other fonds that comprise LAC's $28 \mathrm{~mm}$ collection are given in my spreadsheet. Their Histories are different, and many of the $28 \mathrm{~mm}$ films that are attached to them were in smaller numbers and do not have the same level of information as the large Graphic Consultants fonds. More research is needed to trace these histories leading to LAC than can be encompassed by this thesis. 


\section{Chapter 4: The Spread Sheet: $28 \mathrm{~mm}$ film at LAC}

I began researching the $28 \mathrm{~mm}$ collection by first locating the fonds the films were part of. This was done using MISACS. LAC's audiovisual database MISACS is one of three databases used at LAC and designed for describing audiovisual and sound objects at an item level. These item level descriptions then become attached to the fonds they belong to through the database MIKAN. Navigating the numerous databases presents a problem for public researchers:

"Because of the complexity and age of LAC's descriptive database, the audiovisual item-level descriptions are currently segregated from their fonds or collection descriptions in LAC's Archives Search database." ${ }^{87}$ If a researcher knew the name of the fonds that contained the $28 \mathrm{~mm}$ films, searching that fonds on the archival search database would show the scope and content of that fonds, but not the individual item level descriptions of the films that comprise the fonds. The researcher could search the fonds name in the Film, Video, and Sound database which would give the items within the fonds, but not their scope and content. Generally speaking, the 'scope and content' means the number of reels of film with the fonds and a brief description that is an overview of what is contained in the fonds plus a history of the collection before entering LAC if that is known. If the researcher knew the title of the film alone and not the fonds it is part of, the search of the AV database would show the item level description and not the large custodial history. The custodial history may not be of use to all researchers, but it informs them of how the collection came to be before entering the archive.

The individual AV records are searchable by the public through the, "Film, Video and Sound database" or the "Collections Search" database. Having access to the backend of MISCAS allowed me more search options than available to the public, the most important being able to

\footnotetext{
${ }^{87}$ Cook, "Shrouded History," 147.
} 
search the physical properties such as gauge. LAC's public-facing database lacks the option to search physical properties, such as film gauge, and presents a big problem for researchers of the $28 \mathrm{~mm}$ collection. Without their knowing the titles of the films, the fonds names, or other intellectual information, the only way researchers would be able to search the $28 \mathrm{~mm}$ films would by searching a physical characteristic such as the gauge. The front facing database only searches intellectual properties such as, title, data, accession number, item number (ISN), production credit, etc. ${ }^{88}$ A comparison of searching in the two forms of the database highlights this search problem. Searching, "28mm" in the keyword field on the front facing, "Film, Video and Sound" database and limiting the results to only film, yields a search result of 88 titles. ${ }^{89}$ Records that appear within this search are ones that contain " $28 \mathrm{~mm}$ " either in the description or the notes field. Searching " $28 \mathrm{~mm}$ " in the keyword field on the front-facing "Collection Search" database yields even fewer results with just eight. ${ }^{90}$ Searching $28 \mathrm{~mm}$ in the physical description section of the backend of MISACS yields the full 1,135 results.

Having located all the $28 \mathrm{~mm}$ films in LAC's database using MISCAS, I began to organize the films in an Excel spreadsheet chronologically by accession number. The accession number does not necessarily match the year the films were brought into LAC, but it is close. Organizing the films by accession number as opposed to say, alphabetically by title or by

\footnotetext{
88 “Search-Film, Video, and Sound database," Library and Archives Canada, accessed 2 June 2019, http://www.baclac.gc.ca/eng/discover/films-videos-sound-recordings/film-video-sound-database/Pages/search.aspx

89،"28mm - Film, Video, and Sound database results," Library and Archives Canada, accessed 2 June 2019 , http://www.bac-lac.gc.ca/eng/discover/films-videos-sound-recordings/film-video-sounddatabase/Pages/list.aspx?k=28mm\&MediaEn=Film\&

90“28mm - Collection Search - Film, Video, and Sound database results," Library and Archive Canada, accessed 2 june 2019, http://www.baclac.gc.ca/eng/collectionsearch/Pages/collectionsearch.aspx?DataSource=Archives|FilVidAndSou\&q=28mm\&Operat or $1=$ AND\&Operator_2 $=$ AND\&MediaCode $=\mathrm{F} \&$
} 
production date, also meant that these films visually remain attached to their fonds on the spreadsheet. I then organized my spreadsheet to have the following columns:

- IDCISN: the unique identification number given to all items and searchable in the advanced search section of the Film, Video, and Sound database

- Fonds Name

- Accession number (Acc. Number)

- Series

- Title or given title in [square brackets]

- Date or approximate date signified with ? following AACR2 standard

- Production country (Prd. Country)

- Production company (Prd. Company)

- Sponsor

- Distribution company

- Notes, including such things as whether or not the original print was tinted/toned

I made the choice not to have a column for a description of the film for two reasons. First, the aim of this project is not to better describe these films - most of them have been well described - but to bring these films together as a coherent collection and make these titles accessible for researchers. Second, to streamline the spreadsheet and make it cleaner and easier to view. In my notes section, however, I did make note of the films that I came across that had little to no description. Titles are a searchable field on LAC's database and therefore researchers wanting further information beyond what the title provides can search the title and see the full description. 


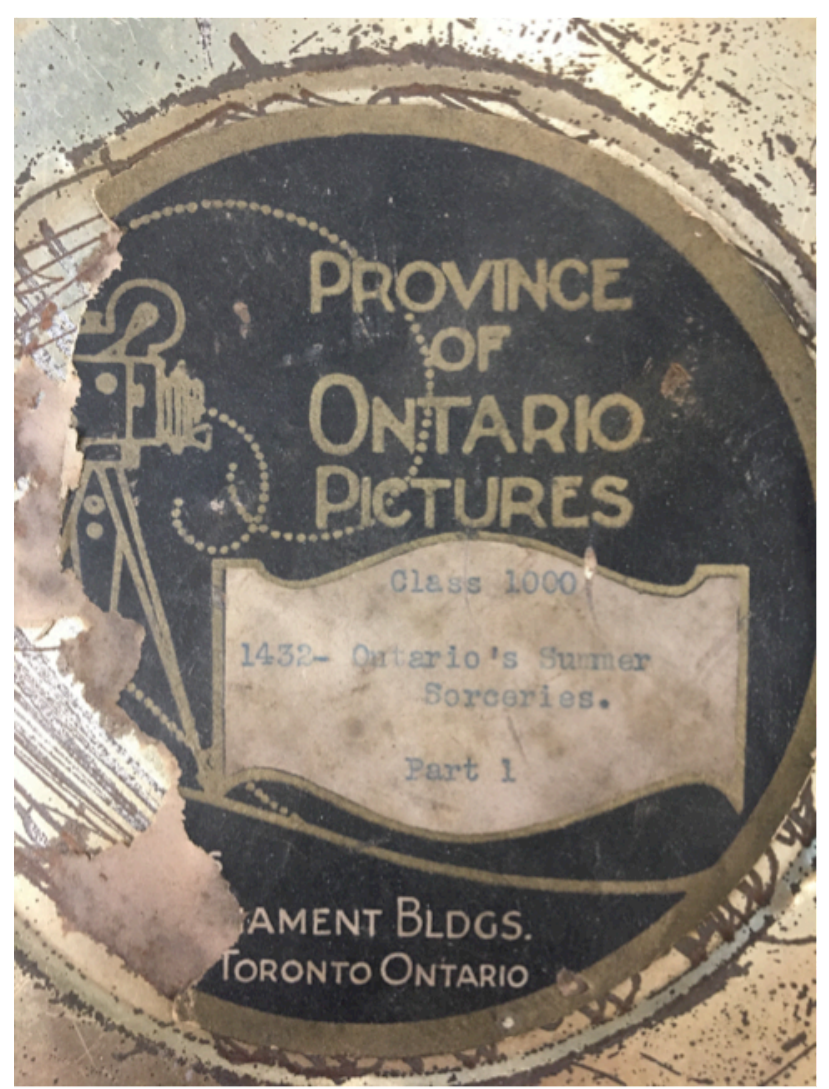

Figure 2. Ontario Motion Picture Bureau (OMPB) original film can with 'Province of Ontario Pictures' written as production company.
Recording the information into the

spreadsheet to accurately display how

information in the LAC database is recorded

did present some issues, specifically for the

production companies. I found that with time,

archivist use and different database systems,

there were a number of different ways the same

production company was recorded.

Furthermore, the OMPB itself often used a

different name on the film and on its the

original can which added to the inconsistencies

created b y the production company (Fig. 2).

An example of such inconsistencies is seen

with three Pathéscope of Canada Films. The first, Wool from the Fleece to the Finished Product:

[incomplete], lists the production company as Pathéscope of Canada Limited. ${ }^{91}$ Another film of

the same fonds, Canadian National Exhibition : [incomplete] lists the production company as

Pathéscope. ${ }^{92}$ A third of the same fonds, Ayrshires at the Canadian National Exhibition, Toronto

lists the production company as Pathéscope of Canada Ltd. ${ }^{93}$ When using the spreadsheet to

draw statistics about how many films were produced by different production companies, each of

91 "Wool from the Fleece to the Finished Product: [incomplete], Library and Archives Canada, accessed 13 June

2019, http://www.bac-lac.gc.ca/eng/discover/films-videos-sound-recordings/film-video-sound-

database/Pages/item.aspx?IdNumber $=416334$

92 “Canadian National Exhibition: [incomplete], Library and Archives Canada, accessed 13 June 2019,

http:/www.bac-lac.gc.ca/eng/discover/films-videos-sound-recordings/film-video-sound-

database/Pages/list.aspx?IdNumber $=460334 \&$

93 “Ayrshires at the Canadian National Exhibition, Toronto," Library and Archives Canada, accessed 13 June 2019 , http://www.bac-lac.gc.ca/eng/discover/films-videos-sound-recordings/film-video-sound-

database/Pages/item.aspx?IdNumber $=93350$ 
the many variations of Pathéscope of Canada would show up as separate production companies. Uploading such data to a database would mean condoning the error: the variations in a single production company name would show up as different companies. I therefore made the choice on the spreadsheet to make consistent all the Canada productions as Pathéscope of Canada, E\&PB, and OMPB. I did the same for French productions that are generally inputted as Pathè: I choose to use Pathé-Frères, the original company name. Due to the size of the collection and the limited time I had to work with it, however, I was not able to standardise all the production companies with this large collection, so I focused specifically on the Canadian companies. By doing this I am not suggesting that LAC should standardize these entries in the same way I have, this is just for the spreadsheet attached to this paper.

MISCAS uses AACR2 for its descriptive standard. For audiovisual items, AACR2 states that the main sources of information for the archivist should be first, the item itself and second the container that holds the item. ${ }^{94}$ Following these standards would have added to the many variations on the names of production companies for these films. Moreover, due to the age of the films, a number of them are missing titles which generally will list the production company and sponsor. In order to determine a production company, the archivist might have used the film can label, which clearly shows variations with how at least the OMPB titled itself, that held the film to source information or, in the case of the Graphic Consultants fonds, the textual material that the company had compiled which could have been inaccurate.

The unusual and small amount of extant $28 \mathrm{~mm}$ films means that $28 \mathrm{~mm}$ gates for film scanners are limited. As of this writing, LAC does not have a $28 \mathrm{~mm}$ gate for its film scanners. In order to preserve these films by transferring them to a more stable polyester stock and then

\footnotetext{
${ }^{94}$ The Concise AACR2 Fourth edition, ed. Michael Gorman, Ottawa: Canadian Library Association, 2004. https://epdf.pub/the-concise-aacr2.html
} 
scanning them, the preservation branch is optically printing the $28 \mathrm{~mm}$ films to $35 \mathrm{~mm}$ black and white internegative stock. The problem with this preservation strategy is that any colour of the films is lost. Because of this lack, I found it important to describe in my notes whether the films were tinted or toned and in what colours where possible. I used the original description to determine the colour of the film tint or toning. 


\section{Chapter 5: Results}

\section{The Statistics}

Once I created the spreadsheet in a way that reflected both the original records and allowed further data to be generated, I was able to use the spreadsheet to better understand this $28 \mathrm{~mm}$ collection. As LAC's website states, "the collection is the most significant record of government produced films." 95 Of the total 1,135 films, 591 titles (just over half) were government-produced in Canada. Of the Canadian films that make up the collection, 188 were produced by Pathéscope of Canada, most of which were sponsored by the OMPB. 200 were produced by the OMPB, representing the shift from sponsoring production companies to producing their own films in 1923. The remaining Canadian productions are in much smaller numbers: 57 produced by Filmcraft Industries, 23 by the E\&PB, and a total of 10 between the Saskatchewan Government Motion Picture Branch and Regina Films Limited. The rest of the collection is made up of 196 films from the US, 160 from France, 36 from the United Kingdom and, finally, 131 films that do not have a known country of production spread over 13 different fonds. Further research into the latter is needed to better complete our understanding of this collection.

\section{Interesting Discoveries}

This research project was about consolidating the $28 \mathrm{~mm}$ titles, beginning to understand the scope of LAC's collection of $28 \mathrm{~mm}$ film, and ultimately providing a means of access for researchers. But my research brought some unexpected results. LAC is currently undergoing a

\footnotetext{
95 "Preservation Activities," Library and Archives Canada, http://www.bac-lac.gc.ca/eng/aboutus/preservation/Pages/preservation-activities.aspx\#g
} 
mass digitization project titled "We are Here: Sharing Stories." 96 This project aims to digitize and make available the extensive amount of material that contains First Nations, Inuit, and Métis Nation content. While consolidating the $28 \mathrm{~mm}$ collection I came across a number of films that clearly related to "We are Here: Sharing Stories" but unknown to that project. Two of the films produced by the OMPB titled, Indian Pow-Wow (1925) and The Romance of Huronia [1922/1923], depict First Nations groups of Ontario performing staged events. While undoubtedly depicted with a colonialist view of the "exotic" to be shown to European audiences, the protagonists of these films, made sometime in the mid 1920s, are nevertheless visual records of First Nations groups and people some 100 years ago. Likewise, the films, The Carrier Indians of British Columbia [1923/1927] and Coast Salish Indians of British Columbia (1928). Both were produced by the National Museum of Canada, established by the Department of Mines around the mid 1920s.

Another LAC initiative, 'Project Naming', part of LAC's Indigenous Heritage undertakings, seeks to identify people and places in LAC collections related to indigenous peoples. This initiative, "adds a more personal layer to the descriptions and the appropriate identification of people who were originally labelled in general, harmful and prejudicial terms." ${ }^{97}$ The subject description of both Indian Pow-Wow and The Romance of Huronia certainly reflect such general and prejudicial terms and could be reviewed and updated. More interesting is the title With the Plowmen at Brampton, an OMPB film that is part of a series covering an annual plowing competition in Brampton, Ontario. The accompanying archivist's note states, "one of [event's] most interesting features was the large numbers of Indian Farmers present, many of

\footnotetext{
96 "We are Here sharing Stores," Library and Archives Canada, accessed June 3, 2019, http://www.baclac.gc.ca/eng/discover/aboriginal-heritage/initiatives/Pages/stories.aspx

97 "Project Naming," Library and Archives Canada, accessed June 3, 2019, http://www.baclac.gc.ca/eng/discover/aboriginal-heritage/project-naming/Pages/introduction.aspx
} 
whom were prize winners." ${ }^{98}$ While films like Indian Pow-Wow and The Romance of Huronia were created to appeal to a romanticized colonial image of First Nations' culture, With the Plowmen at Brampton serves inadvertently to present the First Nations people in an un-romantic, un-exotic reality. Less culturally significant but still historically important and rare are two films printed on Kodak $28 \mathrm{~mm}$ stock rather than the more common $16 \mathrm{~mm}$ stock, Cream Grading in Saskatchewan and [Dairy Farming Industry in Saskatchewan]. The 28mm stock has edge codes that read, "EASTMAN SAFETY," followed by two symbols that relate to the year of production.

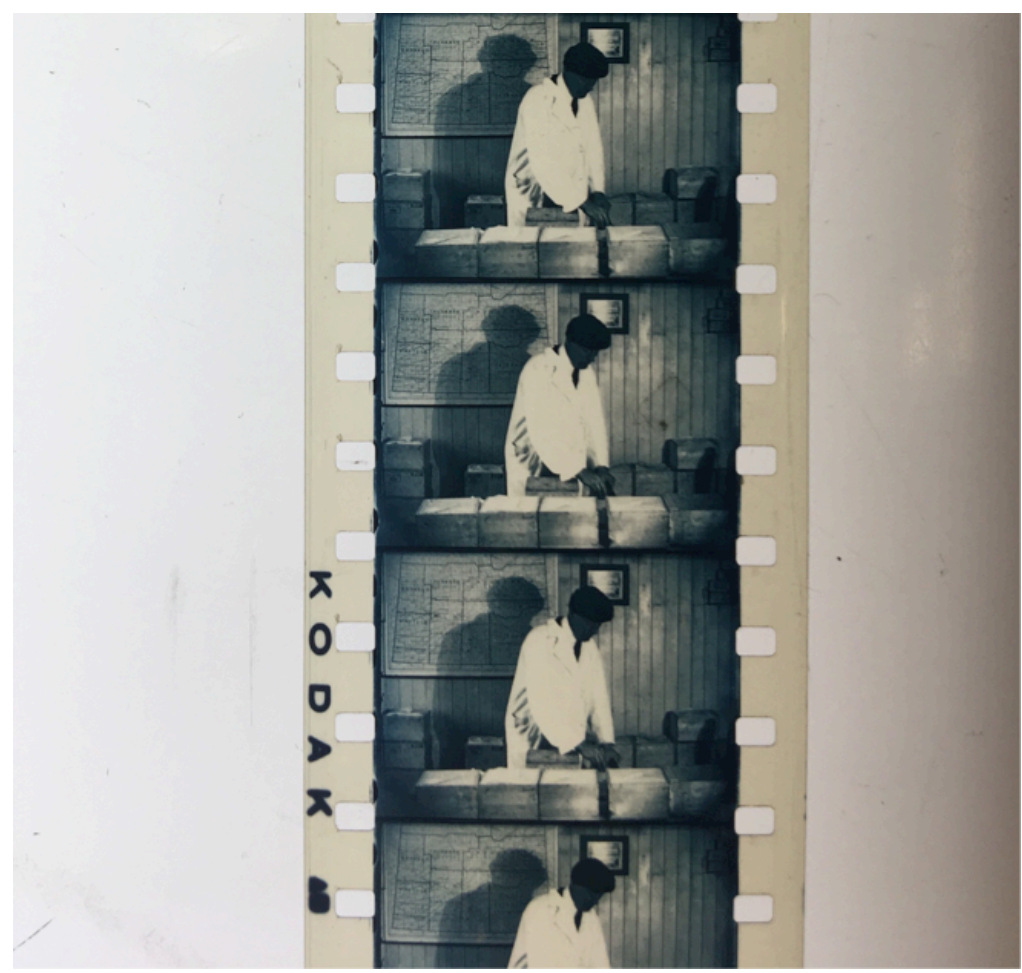

Figure 3. Cream Grading in Saskatchewan printed on Eastman Kodak 28mm stock with edge code symbols displaying a year of 1924.
This system of marking the film

with symbols representing the year

it was printed began in Rochester

in 1916. Film manufactured in

Canada did not begin being marked with symbols until $1925 .{ }^{99}$ The edge code of Cream Grading in Saskatchewan, which LAC assigned a year begin being marked with symbols until $1925 .^{100}$ The edge code of Cream Grading

\footnotetext{
98 “With the Plowmen at Brampton," Library and Archives Canada, accessed, June 3 2019, http://www.baclac.gc.ca/eng/discover/films-videos-sound-recordings/film-video-sounddatabase/Pages/item.aspx?IdNumber $=192040$

${ }^{99}$ Harold Brown, Physical Characteristics of Early Films as Aids to Identification, (Brussels: FIAF, 1990), 13-17, quoted in "Edge Codes and Identification," Filmcolors accessed August 11, 2019, https://filmcolors.org/timelineentry/16668/\#/infobox/16668-44.

${ }^{100}$ Harold Brown, Physical Characteristics of Early Films as Aids to Identification, (Brussels: FIAF, 1990), 13-17, quoted in "Edge Codes and Identification," Filmcolors accessed August 11, 2019, https://filmcolors.org/timelineentry/16668/\#/infobox/16668-44.
} 
in Saskatchewan, which LAC assigned a year range of 1920-1929, actually dates the stock to 1924 (Fig. 3). The edge code of the second film, [Dairy Farming Industry in Saskatchewan] which LAC also dates 1920-1929, dates the stock to 1925. Although the edge codes do not guarantee that either of these films were produced in 1924 or 1925 , they do begin to narrow down the range of production. Given how popular the $28 \mathrm{~mm}$ format was, it is safe to say that these films would have been printed 1925 or fairly close to that date. Curiously, previous research understood Pathé to be the only manufacture of $28 \mathrm{~mm}$ film globally. Film historian and archivist Paolo Cherchi Usai points out that Pathéscope films printed in the United States after 1917 had three perforations on each side but does not mention that it was Kodak that was supplying the $28 \mathrm{~mm}$ stock. $3^{101}$
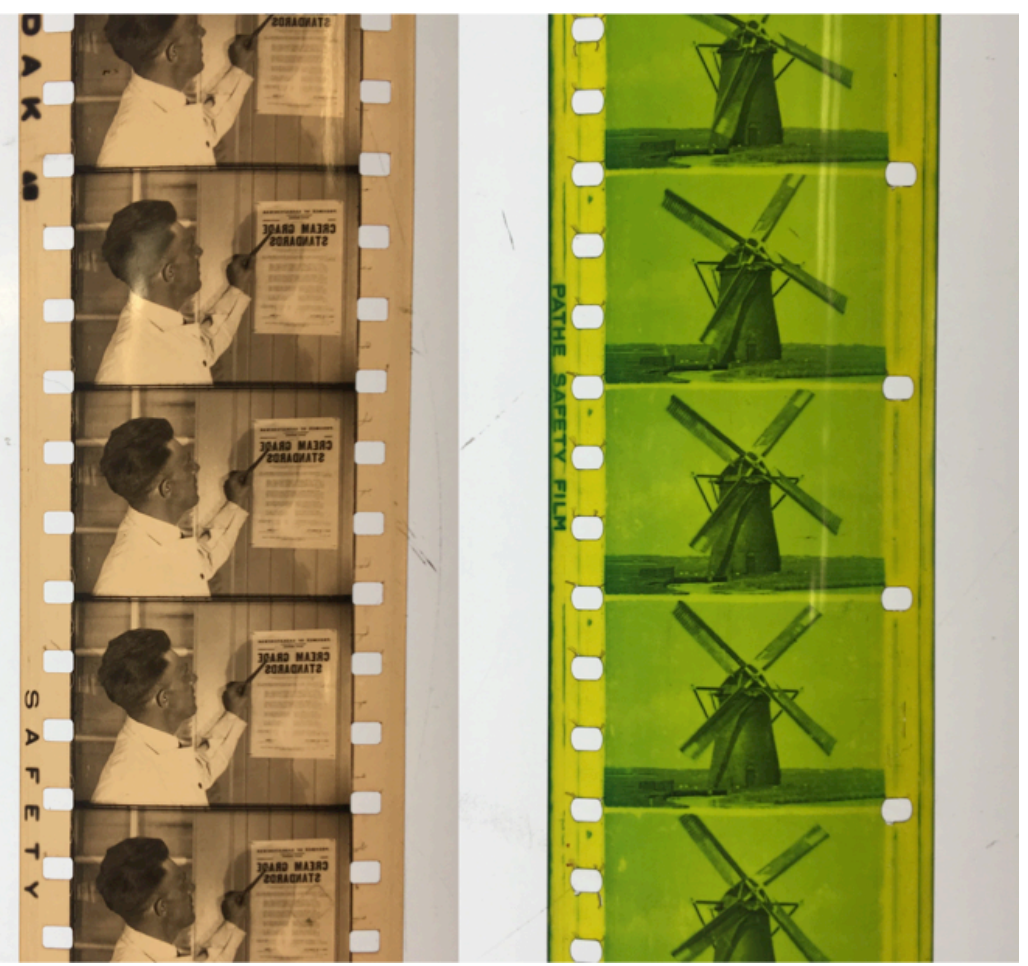

Figure 4. Side by side of [Dairy Farming Industry in Saskatchewan] (left) on Kodak stock and [Wooden Shoes] on Pathé stock. Note the difference in both perforation shape and pattern.

The existence of the Kodak $28 \mathrm{~mm}$ stock at LAC suggests otherwise. One could surmise that because Kodak stock used 3 perforations per side, unlike Pathé stock, it was both compatible

${ }^{101}$ Cherchi Usai, Silent Cinema, 6. 
with the $28 \mathrm{~mm}$ Pathé KOK projector system, but different enough to perhaps avoid any patent infringements (Fig. 4). Also notable are the different perforation shapes. The Kodak stock uses the perforation shape commonly known as the, "Kodak Standard." This shape was introduced by Kodak in 1924 and used only on their positive prints. ${ }^{102}$ The Pathé stock uses a perforation shape unique to Pathé from 1905 to the late 1920s with slightly rounded corners compared to the Kodak. $^{103}$

\section{The intersection between amateur equipment and professional industry}

Pathé-Frères marketed the Pathé KOK and $28 \mathrm{~mm}$ safety stock as an alternative to theatrical film stocks and equipment, an option for those wealthy enough to afford it. During the 1920 s, journals such as Movie Makers continually emphasised that $28 \mathrm{~mm}$ film was a short-lived precursor to Kodak's $16 \mathrm{~mm}$, the long-lasting favourite of amateur filmmakers. But in the same vein as Gerald Graham's proposal that the development of Canadian film technology is directly linked to its evolution as a nation, I would suggest that the importance of Pathé $28 \mathrm{~mm}$ in Canada was not as a format for wealthy amateurs but rather as a format for professional motion picture bureaux to disseminate their films. While there is no doubt that many wealthy Europeans immigrants were living in Canada during this time, their small and dispersed population meant that those who were able to afford the expensive luxury of owning a Pathé KOK projector and film library were very few. The large number of American educational pictures present in the LAC $28 \mathrm{~mm}$ collection suggests that many American companies used the Pathé $28 \mathrm{~mm}$ format for similar distribution means. In Canada, in other words, the short-lived amateur format flourished primarily in the hands of professional picture companies and not in the hands of wealthy citizens.

\footnotetext{
102 Ibid., 3.

103 Ibid.
} 


\section{Conclusion}

The goal of this thesis was formulated as a way of providing a means of access to LAC's collection of $28 \mathrm{~mm}$ films. While titles are available when searched on LAC website, the issues I have identified with the databases make it difficult to locate these films. LAC promotes these unique $28 \mathrm{~mm}$ films as one complete collection yet provides no means of access to these films. Furthermore, the short descriptions on the LAC website of the films in its $28 \mathrm{~mm}$ collection hardly does justice to the collection, inform researchers what makes up the collection, or provide the history of its provenance. By listing these films along with their accession numbers, titles, and other searchable information, my research will allow other researchers interested in pre-NFB Canadian film or unusual formats to explore this collection and expand the current understanding of its content. Furthermore, since only around half the collection is comprised of Canadianproduced films, my list should allow researchers outside of Canada access to titles that might previously have been unknown. Ultimately having these films on a searchable online database would be the easiest way of providing access to these titles. Following the example of other online databases, the incorporation of information such as the filming locations could be used to create a map of shooting locations both in Canada and globally. This is one of many possibilities attending to the exploitation of this collection. I hope that my list will contribute to expanded

research of the history, content, productions, and knowledge of early Canadian film in the $28 \mathrm{~mm}$ format. 


\section{Appendix A: Library and Archives Canada Comprehensive 28mm Film List}

\begin{tabular}{|c|c|c|c|c|c|c|c|c|c|c|}
\hline IDCISN & Fonds Name & $\begin{array}{l}\text { Accession } \\
\text { Number }\end{array}$ & Series & Title or [given title] & $\begin{array}{c}\text { Date or } \\
\text { approximate } \\
\text { date }\end{array}$ & $\begin{array}{c}\text { Prd. } \\
\text { Country } \\
\end{array}$ & Prd. Company & Sponsor & $\begin{array}{c}\text { Distribution } \\
\text { Company } \\
\end{array}$ & Notes \\
\hline & & \multicolumn{2}{|c|}{$\begin{array}{c}\text { University of British } \\
\text { Columbia }\end{array}$} & & & & & & & \\
\hline \multirow[t]{2}{*}{140375} & $\begin{array}{c}\text { University of } \\
\text { British } \\
\text { Columbia } \\
\end{array}$ & 1966-0015 & & $\begin{array}{l}\text { Nation Building in } \\
\text { Saskatchewan : } \\
\text { The Ukrainians }\end{array}$ & 1921 & $\mathrm{cn}$ & $\begin{array}{l}\text { Pathescope of } \\
\text { Canada }\end{array}$ & & & \\
\hline & & $\begin{array}{l}\text { University } \\
\text { of Guelph }\end{array}$ & & & & & & & & \\
\hline \multirow[t]{2}{*}{467686} & $\begin{array}{c}\text { University of } \\
\text { Guelph }\end{array}$ & 1966-0016 & & $\begin{array}{c}\text { The European } \\
\text { Corn Borer }\end{array}$ & 1922 & $\mathrm{cn}$ & $\begin{array}{c}\text { Filmcraft } \\
\text { Industries Ltd. ; } \\
\text { OMPB }\end{array}$ & $\begin{array}{c}\text { Department of } \\
\text { Agriculture, } \\
\text { Entomological } \\
\text { Branch; OMPB }\end{array}$ & & \\
\hline & & Sowman, J & & & & & & & & \\
\hline 278583 & Sowman, J. & 1969-0029 & & $\begin{array}{c}\text { [Early Canadian } \\
\text { Children's } \\
\text { Cartoons] } \\
\end{array}$ & $\mathrm{N} / \mathrm{A}$ & $\mathrm{cn}$ & $\begin{array}{c}\text { Canadian Kodak } \\
? \\
\end{array}$ & & & $\begin{array}{c}\text { no } \\
\text { description }\end{array}$ \\
\hline \multirow[t]{2}{*}{51630} & Sowman, J. & 1969-0029 & & $\begin{array}{l}\text { [Princess Patricia's } \\
\text { Canadian Light } \\
\text { Infantry Regiment } \\
\text { Returns to } \\
\text { Canada] } \\
\end{array}$ & 1919 & $\mathrm{cn}$ & $\begin{array}{c}\text { Canadian Kodak } \\
? \\
\end{array}$ & & & \\
\hline & & \multicolumn{2}{|c|}{ National Hockey League } & & & & & & & \\
\hline \multirow[t]{2}{*}{341005} & NHL & 1969-0060 & & Grading Stallions & $1920 / 1929$ & $\mathrm{cn}$ & $\begin{array}{c}\text { Saskatchwan } \\
\text { Government } \\
\text { Bureau of } \\
\text { Publications, } \\
\text { Motion Picture } \\
\text { Branch } \\
\end{array}$ & & & \\
\hline & & \multicolumn{2}{|c|}{ Mackinnon, James Angus } & & & & & & & \\
\hline \multirow[t]{2}{*}{328589} & $\begin{array}{l}\text { Mackinnon, } \\
\text { James Angus }\end{array}$ & 1970-0033 & & $\begin{array}{c}\text { [Education in } \\
\text { Saskatchewan = } \\
\text { Canadianization in } \\
\text { Saskatchewan] } \\
\end{array}$ & 1920/1929? & $\mathrm{cn}$ & $\begin{array}{c}\text { The Department } \\
\text { of Education, } \\
\text { Regina } \\
\text { Saskatchewan }\end{array}$ & & & \\
\hline & & \multicolumn{2}{|c|}{ Graphic Consultants LTD. } & & & & & & & \\
\hline 157730 & $\begin{array}{c}\text { Graphic } \\
\text { Consultants } \\
\text { LTD. }\end{array}$ & $1970-0046$ & & $\begin{array}{c}\text { Victory Loan } 1918 \\
1918 \\
\end{array}$ & 1918 & $\mathrm{cn}$ & $\begin{array}{c}\text { Famous Players- } \\
\text { Lasky }\end{array}$ & $\begin{array}{c}\text { Dominion } \\
\text { Publicity } \\
\text { Committee }\end{array}$ & & \\
\hline 361999 & $\begin{array}{c}\text { Graphic } \\
\text { Consultants } \\
\text { LTD. }\end{array}$ & $1970-0046$ & & $\begin{array}{c}\text { Frenchy Bélanger } \\
\text { versus Ernie Jarvis } \\
\text { in the World's } \\
\text { Flyweight } \\
\text { Championship } \\
\text { Bout } \\
\end{array}$ & 1927 & $\mathrm{cn}$ & $\mathrm{N} / \mathrm{A}$ & & & $\begin{array}{c}\text { no } \\
\text { description } \\
\end{array}$ \\
\hline 376579 & $\begin{array}{c}\text { Graphic } \\
\text { Consultants } \\
\text { LTD. }\end{array}$ & $1970-0046$ & $\begin{array}{l}\text { Cameraring } \\
\text { in Canada }\end{array}$ & $\begin{array}{c}\text { Where Firemen } \\
\text { Grow Wings : Fire } \\
\text { Fighting With } \\
\text { Aeroplanes }\end{array}$ & 1922/1923? & $\mathrm{cn}$ & $\begin{array}{c}\text { Filmcraft } \\
\text { Industries Ltd. }\end{array}$ & OMPB & & \\
\hline \multirow[t]{2}{*}{533986} & $\begin{array}{c}\text { Graphic } \\
\text { Consultants } \\
\text { LTD. }\end{array}$ & $1970-0046$ & & $\begin{array}{c}\text { [Canadian Forces } \\
\text { in France June } \\
\text { 1916: Battle for } \\
\text { Verdun] }\end{array}$ & $1916 / 1917$ & uk & N/A & & & \\
\hline & & $\begin{array}{c}\text { Bird, } \\
\text { Richard } \\
\text { "Dick" }\end{array}$ & & & & & & & & \\
\hline \multirow[t]{2}{*}{355018} & $\begin{array}{c}\text { Bird, Richard } \\
\text { "Dick" }\end{array}$ & $1971-0017$ & & $\begin{array}{c}\text { Education Day at } \\
\text { Melfort = } \\
\text { [Education Day } \\
\text { Parade in Melfort } \\
\text { Saskatchewan] }\end{array}$ & $1927-06$ & $\mathrm{cn}$ & $\begin{array}{l}\text { Pathescope of } \\
\text { Canada }\end{array}$ & & & $\begin{array}{l}\text { Tinted } \\
\text { unknown } \\
\text { colour } \\
\end{array}$ \\
\hline & & \multicolumn{2}{|c|}{ Graphic Consultants LTD. } & & & & & & & \\
\hline
\end{tabular}




\begin{tabular}{|c|c|c|c|c|c|c|c|c|c|c|}
\hline 365669 & $\begin{array}{c}\text { Graphic } \\
\text { Consultants } \\
\text { LTD. }\end{array}$ & 1972-0105 & $\begin{array}{l}\text { Paramount } \\
\text { Pictograph }\end{array}$ & $\begin{array}{l}\text { Ice : Natural and } \\
\text { Made-to-Order }\end{array}$ & $1920 / 1929$ & us & Paramount & & & $\begin{array}{l}\text { Tinted } \\
\text { orange }\end{array}$ \\
\hline 415453 & $\begin{array}{c}\text { Graphic } \\
\text { Consultants } \\
\text { LTD. } \\
\end{array}$ & 1972-0105 & & $\begin{array}{l}\text { Museum of } \\
\text { Oceanography at } \\
\text { Monte Carlo }\end{array}$ & 1920/1924? & $\mathrm{fr}$ & Pathe Freres & & & \\
\hline 103649 & $\begin{array}{c}\text { Graphic } \\
\text { Consultants } \\
\text { LTD. }\end{array}$ & $1972-0105$ & $\begin{array}{l}\text { Shadow } \\
\text { Laugh }\end{array}$ & $\begin{array}{l}\text { One Bad Knight : } \\
\text { A Mediaeval } \\
\text { Romance } \\
\end{array}$ & 1927 & $\mathrm{cn}$ & Bryant Fryer? & & & \\
\hline 104810 & $\begin{array}{c}\text { Graphic } \\
\text { Consultants } \\
\text { LTD. }\end{array}$ & 1972-0105 & & Ontario's Pulp & $1923 ?$ & $\mathrm{cn}$ & OMPB & & & \\
\hline 104812 & $\begin{array}{c}\text { Graphic } \\
\text { Consultants } \\
\text { LTD. }\end{array}$ & $1972-0105$ & & $\begin{array}{l}\text { Construction of a } \\
\text { Waterbound } \\
\text { Macadam Road }\end{array}$ & 1917 ? & $\mathrm{cn}$ & $\begin{array}{l}\text { Pathescope of } \\
\text { Canada }\end{array}$ & OMPB & & \\
\hline 104813 & $\begin{array}{c}\text { Graphic } \\
\text { Consultants } \\
\text { LTD. }\end{array}$ & $1972-0105$ & & $\begin{array}{c}\text { The Construction } \\
\text { of a Gravel Road } \\
\text { = The Gravel } \\
\text { Road }\end{array}$ & 1919 & $\mathrm{cn}$ & $\begin{array}{c}\text { Pathescope of } \\
\text { Canada }\end{array}$ & OMPB & & \\
\hline 104814 & $\begin{array}{c}\text { Graphic } \\
\text { Consultants } \\
\text { LTD. }\end{array}$ & $1972-0105$ & & $\begin{array}{c}\text { Proportioning } \\
\text { Concrete } \\
\text { Mixtures. Mixing } \\
\text { and Placing } \\
\text { Concrete }\end{array}$ & $1920 / 1925$ & $\mathrm{cn}$ & $\begin{array}{l}\text { Pathescope of } \\
\text { Canada }\end{array}$ & $\begin{array}{c}\text { OMPB - } \\
\text { Courtesy of } \\
\text { Canada Cement } \\
\text { Compnay } \\
\text { Limited }\end{array}$ & & \\
\hline 104818 & $\begin{array}{c}\text { Graphic } \\
\text { Consultants } \\
\text { LTD. } \\
\end{array}$ & 1972-0105 & & $\begin{array}{c}\text { The Construction } \\
\text { of Tar Macadam } \\
\text { Road }\end{array}$ & $1917 ?$ & $\mathrm{cn}$ & $\begin{array}{c}\text { Pathescope of } \\
\text { Canada }\end{array}$ & OMPB & OMPB & \\
\hline 104820 & $\begin{array}{c}\text { Graphic } \\
\text { Consultants } \\
\text { LTD. }\end{array}$ & 1972-0105 & & $\begin{array}{l}\text { Treaders of the } \\
\text { Broad Highways }\end{array}$ & 1924 & $\mathrm{cn}$ & OMPB & & & \\
\hline 104821 & $\begin{array}{c}\text { Graphic } \\
\text { Consultants } \\
\text { LTD. }\end{array}$ & 1972-0105 & & $\begin{array}{c}\text { Decazeville and } \\
\text { Its Metallurgical } \\
\text { Works } \\
\end{array}$ & $1920 / 1925$ & $\mathrm{fr}$ & Pathe & & OMPB & Tinted red \\
\hline 104824 & $\begin{array}{c}\text { Graphic } \\
\text { Consultants } \\
\text { LTD. } \\
\end{array}$ & $1972-0105$ & & $\begin{array}{c}\text { Hydro } \\
\text { Development on } \\
\text { the Severn River }= \\
\text { Power } \\
\text { Development on } \\
\text { the Severn River }\end{array}$ & $1920 / 1922$ & $\mathrm{cn}$ & $\begin{array}{c}\text { Filmcraft } \\
\text { Industries Ltd. }\end{array}$ & OMPB & & \\
\hline 104825 & $\begin{array}{c}\text { Graphic } \\
\text { Consultants } \\
\text { LTD. }\end{array}$ & 1972-0105 & & $\begin{array}{l}\text { Tapping Niagara's } \\
\text { Power Resources }\end{array}$ & $1922 ?$ & $\mathrm{cn}$ & $\begin{array}{c}\text { Filmcraft } \\
\text { Industries Ltd. }\end{array}$ & OMPB & & \\
\hline 104826 & $\begin{array}{c}\text { Graphic } \\
\text { Consultants } \\
\text { LTD. }\end{array}$ & 1972-0105 & & $\begin{array}{c}\text { Building a Modern } \\
\text { Grain Elevator }\end{array}$ & 1923 & $\mathrm{cn}$ & $\begin{array}{c}\text { Filmcraft } \\
\text { Industries Ltd. }\end{array}$ & OMPB & & \\
\hline 104833 & $\begin{array}{c}\text { Graphic } \\
\text { Consultants } \\
\text { LTD. }\end{array}$ & $1972-0105$ & & $\begin{array}{c}\text { The Welland Ship } \\
\text { Canal }\end{array}$ & 1925 & $\mathrm{cn}$ & OMPB & & & \\
\hline 104846 & $\begin{array}{c}\text { Graphic } \\
\text { Consultants } \\
\text { LTD. }\end{array}$ & 1972-0105 & & $\begin{array}{c}\text { Power } \\
\text { Development on } \\
\text { the Nipigon River } \\
=\text { Construction of } \\
\text { the Hydro } \\
\text { Undertaking at } \\
\text { Cameron Falls } \\
\end{array}$ & $1920 / 1922$ & $\mathrm{cn}$ & $\begin{array}{c}\text { Filmcraft } \\
\text { Industries Ltd. }\end{array}$ & OMPB & & \\
\hline 108889 & $\begin{array}{c}\text { Graphic } \\
\text { Consultants } \\
\text { LTD. }\end{array}$ & 1972-0105 & & Someone at Home & 1925 & $\mathrm{cn}$ & OMPB & & & \\
\hline 108890 & $\begin{array}{c}\text { Graphic } \\
\text { Consultants } \\
\text { LTD. } \\
\end{array}$ & $1972-0105$ & & $\begin{array}{l}\text { Air Patrol: } \\
\text { [incomplete] }\end{array}$ & $1925 ?$ & $\mathrm{cn}$ & OMPB & & OMPB & \\
\hline 108891 & $\begin{array}{c}\text { Graphic } \\
\text { Consultants } \\
\text { LTD. }\end{array}$ & 1972-0105 & & Mailing Trouble & 1929 & $\mathrm{cn}$ & OMPB & & & \\
\hline 111046 & $\begin{array}{c}\text { Graphic } \\
\text { Consultants } \\
\text { LTD. }\end{array}$ & 1972-0105 & & $\begin{array}{c}\text { Admiral Jellicoe } \\
\text { Officially Opens } \\
\text { the Canadian } \\
\text { National } \\
\text { Exhibition : } 15000\end{array}$ & 1931 & $\mathrm{cn}$ & OMPB & & & \\
\hline
\end{tabular}




\begin{tabular}{|c|c|c|c|c|c|c|c|c|c|c|}
\hline & & & & $\begin{array}{l}\text { Veterans Pass in } \\
\text { Review on } \\
\text { Warrior's Day }\end{array}$ & & & & & & \\
\hline 111111 & $\begin{array}{c}\text { Graphic } \\
\text { Consultants } \\
\text { LTD. } \\
\end{array}$ & $1972-0105$ & & $\begin{array}{c}\text { Canada Welcomes } \\
\text { Kaye Don : } \\
\text { Citizens of } \\
\text { Toronto Give } \\
\text { Great Ovation to } \\
\text { Empire Hero } \\
\end{array}$ & 1931 & $\mathrm{cn}$ & OMPB & & & \\
\hline 111112 & $\begin{array}{l}\text { Graphic } \\
\text { Consultants } \\
\text { LTD. } \\
\end{array}$ & 1972-0105 & & Breaking the Ice & $1925 ?$ & $\mathrm{cn}$ & OMPB & & & \\
\hline 111113 & $\begin{array}{l}\text { Graphic } \\
\text { Consultants } \\
\text { LTD. }\end{array}$ & $1972-0105$ & & $\begin{array}{l}\text { Help the Blind to } \\
\text { Help Themselves }\end{array}$ & $1927 ?$ & $\mathrm{cn}$ & OMPB & & & \\
\hline 111141 & $\begin{array}{l}\text { Graphic } \\
\text { Consultants } \\
\text { LTD. } \\
\end{array}$ & 1972-0105 & & $\begin{array}{c}\text { Toronto } \\
\text { Welcomes } \\
\text { Governor General }\end{array}$ & 1931 & $\mathrm{cn}$ & OMPB & & & \\
\hline 111143 & $\begin{array}{c}\text { Graphic } \\
\text { Consultants } \\
\text { LTD. }\end{array}$ & 1972-0105 & & $\begin{array}{l}\text { A Visit from } \\
\text { Pharaoh: } \\
\text { [incomplete] }\end{array}$ & $1924 ?$ & $\mathrm{cn}$ & OMPB & & & \\
\hline 111146 & $\begin{array}{l}\text { Graphic } \\
\text { Consultants } \\
\text { LTD. } \\
\end{array}$ & 1972-0105 & & $\begin{array}{c}\text { When Navigation } \\
\text { Ends }\end{array}$ & 1927 & $\mathrm{cn}$ & OMPB & & & \\
\hline 120269 & $\begin{array}{l}\text { Graphic } \\
\text { Consultants } \\
\text { LTD. } \\
\end{array}$ & $1972-0105$ & & $\begin{array}{c}\text { Learning Modern } \\
\text { Industrial } \\
\text { Processes : Central } \\
\text { Technical School } \\
\text { Toronto } \\
\end{array}$ & 1919 & $\mathrm{cn}$ & $\begin{array}{c}\text { Filmcraft } \\
\text { Industries Ltd. }\end{array}$ & OMPB & & \\
\hline 120270 & $\begin{array}{l}\text { Graphic } \\
\text { Consultants } \\
\text { LTD. } \\
\end{array}$ & $1972-0105$ & & $\begin{array}{c}\text { [In Toronto's } \\
\text { Central Technical } \\
\text { School] } \\
\end{array}$ & 1920 & $\mathrm{cn}$ & OMPB & & & \\
\hline 120285 & $\begin{array}{l}\text { Graphic } \\
\text { Consultants } \\
\text { LTD. } \\
\end{array}$ & 1972-0105 & & $\begin{array}{c}\text { Miners in the } \\
\text { Making }\end{array}$ & 1922 & $\mathrm{cn}$ & $\begin{array}{c}\text { Pathescope of } \\
\text { Canada }\end{array}$ & OMPB & & \\
\hline 120360 & $\begin{array}{c}\text { Graphic } \\
\text { Consultants } \\
\text { LTD. }\end{array}$ & $1972-0105$ & & $\begin{array}{l}\text { The Rugged Road } \\
\text { to Learning }\end{array}$ & 1921 & $\mathrm{cn}$ & $\begin{array}{c}\text { Pathescope of } \\
\text { Canada }\end{array}$ & OMPB & & \\
\hline 140490 & $\begin{array}{c}\text { Graphic } \\
\text { Consultants } \\
\text { LTD. } \\
\end{array}$ & 1972-0105 & $\begin{array}{l}\text { Canadian } \\
\text { National } \\
\text { Pictorial } \\
\end{array}$ & $\begin{array}{c}\text { Ottawa : Home of } \\
\text { Canada's } \\
\text { Parliament } \\
\end{array}$ & $1919 / 1920$ & $\mathrm{cn}$ & $\begin{array}{c}\text { Pathescope of } \\
\text { Canada }\end{array}$ & $\begin{array}{c}\text { The Government } \\
\text { of Canada }\end{array}$ & & \\
\hline 147688 & $\begin{array}{c}\text { Graphic } \\
\text { Consultants } \\
\text { LTD. }\end{array}$ & $1972-0105$ & & $\begin{array}{c}\text { Installing a Giant } \\
\text { Generator }\end{array}$ & $1920 / 1922$ & $\mathrm{cn}$ & $\begin{array}{l}\text { Pathescope of } \\
\text { Canada }\end{array}$ & OMPB & & \\
\hline 369903 & $\begin{array}{l}\text { Graphic } \\
\text { Consultants } \\
\text { LTD. }\end{array}$ & 1972-0105 & & $\begin{array}{l}\text { Schoolboy Track } \\
\text { and Field } \\
\text { Championships }\end{array}$ & 1928 & $\mathrm{cn}$ & OMPB & & & \\
\hline 157269 & $\begin{array}{l}\text { Graphic } \\
\text { Consultants } \\
\text { LTD. } \\
\end{array}$ & $1972-0105$ & & $\begin{array}{l}\text { The King's } \\
\text { Highway }\end{array}$ & $1922 / 1923$ & $\mathrm{cn}$ & $\begin{array}{c}\text { Filmcraft } \\
\text { Industries Ltd. }\end{array}$ & OMPB & & \\
\hline 157274 & $\begin{array}{c}\text { Graphic } \\
\text { Consultants } \\
\text { LTD. } \\
\end{array}$ & $1972-0105$ & & A Square Deal & 1926 & $\mathrm{cn}$ & OMPB & & & \\
\hline 157347 & $\begin{array}{c}\text { Graphic } \\
\text { Consultants } \\
\text { LTD. } \\
\end{array}$ & $1972-0105$ & & Temple of Pines & 1930 & $\mathrm{cn}$ & OMPB & & & \\
\hline 158406 & $\begin{array}{l}\text { Graphic } \\
\text { Consultants } \\
\text { LTD. }\end{array}$ & $1972-0105$ & & $\begin{array}{c}\text { When Kappa } \\
\text { Kappa Gamma } \\
\text { Visited Ontario : } \\
\text { [incomplete] }\end{array}$ & 1924 & $\mathrm{cn}$ & OMPB & & & \\
\hline 158408 & $\begin{array}{l}\text { Graphic } \\
\text { Consultants } \\
\text { LTD. } \\
\end{array}$ & 1972-0105 & & In Lumberland & 1923 & $\mathrm{cn}$ & $\begin{array}{c}\text { Filmcraft } \\
\text { Industries Ltd. }\end{array}$ & $\begin{array}{c}\text { Province of } \\
\text { Ontario }\end{array}$ & & \\
\hline 159810 & $\begin{array}{c}\text { Graphic } \\
\text { Consultants } \\
\text { LTD. }\end{array}$ & $1972-0105$ & $\begin{array}{l}\text { Seeing } \\
\text { Canada }\end{array}$ & $\begin{array}{l}\text { Canada's } \\
\text { Metropolis }\end{array}$ & 1921/1922? & $\mathrm{cn}$ & $\begin{array}{c}\text { Exhibits and } \\
\text { Publicity Bureau }\end{array}$ & & & \\
\hline
\end{tabular}




\begin{tabular}{|c|c|c|c|c|c|c|c|c|c|}
\hline 185638 & $\begin{array}{c}\text { Graphic } \\
\text { Consultants } \\
\text { LTD. }\end{array}$ & $1972-0105$ & & $\begin{array}{l}\text { Transport in the } \\
\text { North }\end{array}$ & 1925 & $\mathrm{cn}$ & OMPB & & \\
\hline 185644 & $\begin{array}{l}\text { Graphic } \\
\text { Consultants } \\
\text { LTD. } \\
\end{array}$ & $1972-0105$ & & Your Future Car & 1924 & $\mathrm{cn}$ & OMPB & & \\
\hline 185835 & $\begin{array}{l}\text { Graphic } \\
\text { Consultants } \\
\text { LTD. } \\
\end{array}$ & $1972-0105$ & & $\begin{array}{c}\text { Hot Noonday } \\
\text { Lunches for Rural } \\
\text { Schools } \\
\end{array}$ & 1919 & $\mathrm{cn}$ & $\begin{array}{c}\text { Pathescope of } \\
\text { Canada }\end{array}$ & $\begin{array}{c}\text { Ontario } \\
\text { Department of } \\
\text { Education } \\
\end{array}$ & \\
\hline 185837 & $\begin{array}{l}\text { Graphic } \\
\text { Consultants } \\
\text { LTD. } \\
\end{array}$ & $1972-0105$ & & $\begin{array}{l}\text { The Kemptville } \\
\text { Agricultural } \\
\text { School }\end{array}$ & 1925 & $\mathrm{cn}$ & OMPB & & \\
\hline 185838 & $\begin{array}{l}\text { Graphic } \\
\text { Consultants } \\
\text { LTD. } \\
\end{array}$ & $1972-0105$ & & The Harvest & $1920 / 1925$ & $\mathrm{cn}$ & $\mathrm{N} / \mathrm{A}$ & $\mathrm{N} / \mathrm{A}$ & \\
\hline 185839 & $\begin{array}{l}\text { Graphic } \\
\text { Consultants } \\
\text { LTD. } \\
\end{array}$ & $1972-0105$ & & $\begin{array}{c}\text { Business } \\
\text { Opportunity on the } \\
\text { Farm } \\
\end{array}$ & 1920/1922? & $\mathrm{cn}$ & $\begin{array}{l}\text { Pathescope of } \\
\text { Canada }\end{array}$ & $\begin{array}{c}\text { Ontario } \\
\text { Department of } \\
\text { Agriculture } \\
\end{array}$ & \\
\hline 188460 & $\begin{array}{c}\text { Graphic } \\
\text { Consultants } \\
\text { LTD. } \\
\end{array}$ & $1972-0105$ & $\begin{array}{l}\text { Canadian } \\
\text { Official War } \\
\text { Films } \\
\end{array}$ & $\begin{array}{l}\text { In Action with our } \\
\text { Canadian Troops }\end{array}$ & $1917 ?$ & $\mathrm{cn}$ & $\begin{array}{l}\text { British Topical } \\
\text { Committee for } \\
\text { War Films }\end{array}$ & War Office & \\
\hline 188462 & $\begin{array}{l}\text { Graphic } \\
\text { Consultants } \\
\text { LTD. } \\
\end{array}$ & $1972-0105$ & & $\begin{array}{c}\text { After the Retreat } \\
\text { of the German } \\
\text { Army = After the } \\
\text { Retreat. } \\
\text { Reconquered } \\
\text { Alsace }\end{array}$ & $1914 / 1918$ & $\mathrm{fr}$ & Pathe Freres & & \\
\hline 192038 & $\begin{array}{l}\text { Graphic } \\
\text { Consultants } \\
\text { LTD. } \\
\end{array}$ & $1972-0105$ & & $\begin{array}{l}\text { The Educational } \\
\text { Playgrounds }\end{array}$ & 1922 & $\mathrm{cn}$ & $\begin{array}{c}\text { Filmcraft } \\
\text { Industries Ltd. }\end{array}$ & OMPB & \\
\hline 192040 & $\begin{array}{l}\text { Graphic } \\
\text { Consultants } \\
\text { LTD. } \\
\end{array}$ & $1972-0105$ & & $\begin{array}{l}\text { With the Plowmen } \\
\text { at Brampton }\end{array}$ & $1924 / 1925$ & $\mathrm{cn}$ & OMPB & & $\begin{array}{c}\text { Noted "large } \\
\text { number of } \\
\text { Indian } \\
\text { farmers } \\
\text { present, } \\
\text { many of } \\
\text { whom were } \\
\text { prize } \\
\text { winners" } \\
\end{array}$ \\
\hline 192832 & $\begin{array}{l}\text { Graphic } \\
\text { Consultants } \\
\text { LTD. } \\
\end{array}$ & $1972-0105$ & & $\begin{array}{l}\text { Adventures of } \\
\text { Geo. S. Fishog } \\
\end{array}$ & 1930 & $\mathrm{cn}$ & $\begin{array}{c}\text { Ontario Forestry } \\
\text { Branch; Ontario } \\
\text { Motion Picture } \\
\text { Bureau } \\
\end{array}$ & $\begin{array}{c}\text { Ontrario } \\
\text { Forestry } \\
\text { Association } \\
\end{array}$ & \\
\hline 193022 & $\begin{array}{l}\text { Graphic } \\
\text { Consultants } \\
\text { LTD. } \\
\end{array}$ & $1972-0105$ & & Indian Pow-Wow & 1925 & $\mathrm{cn}$ & OMPB & & \\
\hline 193023 & $\begin{array}{l}\text { Graphic } \\
\text { Consultants } \\
\text { LTD. } \\
\end{array}$ & 1972-0105 & & $\begin{array}{c}\text { The Romance of } \\
\text { Huronia }\end{array}$ & $1922 / 1923$ & $\mathrm{cn}$ & $\begin{array}{c}\text { Filmcraft } \\
\text { Industries Ltd. }\end{array}$ & OMPB & \\
\hline 193977 & $\begin{array}{l}\text { Graphic } \\
\text { Consultants } \\
\text { LTD. } \\
\end{array}$ & 1972-0105 & $\begin{array}{l}\text { Shadow } \\
\text { Laugh }\end{array}$ & $\begin{array}{c}\text { Follow the } \\
\text { Swallow : A Story } \\
\text { of the Stone Age }\end{array}$ & 1927 & $\mathrm{cn}$ & Bryant Fryer? & & \\
\hline 194538 & $\begin{array}{l}\text { Graphic } \\
\text { Consultants } \\
\text { LTD. } \\
\end{array}$ & $1972-0105$ & & $\begin{array}{c}\text { Where East Meets } \\
\text { West }\end{array}$ & 1925 & $\mathrm{cn}$ & OMPB & & \\
\hline 194560 & $\begin{array}{l}\text { Graphic } \\
\text { Consultants } \\
\text { LTD. } \\
\end{array}$ & $1972-0105$ & & $\begin{array}{c}\text { The Story of the } \\
\text { Stone }\end{array}$ & 1925 & $\mathrm{cn}$ & OMPB & & \\
\hline 194561 & $\begin{array}{l}\text { Graphic } \\
\text { Consultants } \\
\text { LTD. } \\
\end{array}$ & $1972-0105$ & & $\begin{array}{c}\text { Hamilton } \\
\text { Memorial Service } \\
\text { for the Late Sir } \\
\text { Adam Beck }\end{array}$ & 1925 & $\mathrm{cn}$ & $\mathrm{N} / \mathrm{A}$ & N/A & \\
\hline 199415 & $\begin{array}{l}\text { Graphic } \\
\text { Consultants } \\
\text { LTD. }\end{array}$ & $1972-0105$ & $\begin{array}{l}\text { Seeing } \\
\text { Canada }\end{array}$ & $\begin{array}{c}\text { Fresh from the } \\
\text { Deep }\end{array}$ & 1922 & $\mathrm{cn}$ & $\begin{array}{c}\text { Exhibits and } \\
\text { Publicity Bureau }\end{array}$ & $\begin{array}{c}\text { Fisheries } \\
\text { Branch, } \\
\text { Department of } \\
\text { Marine and } \\
\text { Fisheries } \\
\end{array}$ & \\
\hline
\end{tabular}




\begin{tabular}{|c|c|c|c|c|c|c|c|c|c|c|}
\hline 199434 & $\begin{array}{l}\text { Graphic } \\
\text { Consultants } \\
\text { LTD. } \\
\end{array}$ & $1972-0105$ & & $\begin{array}{c}\text { Tobacco } \\
\text { Production in } \\
\text { Southwestern } \\
\text { Ontario } \\
\end{array}$ & 1920 & $\mathrm{cn}$ & $\begin{array}{c}\text { Ontario } \\
\text { Government } \\
\text { Pictures } \\
\end{array}$ & & & \\
\hline 201473 & $\begin{array}{c}\text { Graphic } \\
\text { Consultants } \\
\text { LTD. } \\
\end{array}$ & $1972-0105$ & & $\begin{array}{c}\text { Where Nature } \\
\text { Smiles }\end{array}$ & 1921 & cn & CGMPB & & & \\
\hline 201474 & $\begin{array}{l}\text { Graphic } \\
\text { Consultants } \\
\text { LTD. } \\
\end{array}$ & $1972-0105$ & & Celery Culture & $1919 ?$ & $\mathrm{cn}$ & $\begin{array}{c}\text { Pathescope of } \\
\text { Canada }\end{array}$ & OMPB & & \\
\hline 201475 & $\begin{array}{l}\text { Graphic } \\
\text { Consultants } \\
\text { LTD. } \\
\end{array}$ & $1972-0105$ & & Dollars and Sense & & us & $\begin{array}{l}\text { Triangle- } \\
\text { Keystone }\end{array}$ & & & \\
\hline 201476 & $\begin{array}{l}\text { Graphic } \\
\text { Consultants } \\
\text { LTD. } \\
\end{array}$ & $1972-0105$ & & $\begin{array}{c}\text { Through Life's } \\
\text { Windows }\end{array}$ & 1919 & us & $\begin{array}{c}\text { Worcester Film } \\
\text { Corporation }\end{array}$ & & OMPB & $\begin{array}{c}\text { Tinted } \\
\text { unknown } \\
\text { colour }\end{array}$ \\
\hline 201477 & $\begin{array}{l}\text { Graphic } \\
\text { Consultants } \\
\text { LTD. } \\
\end{array}$ & $1972-0105$ & & Kelly's Cruise & $1929 ?$ & $\mathrm{cn}$ & $\begin{array}{c}\text { Ontario Motion } \\
\text { Picture Bureau }\end{array}$ & $\begin{array}{c}\text { Trent Waterway } \\
\text { Development } \\
\text { Association }\end{array}$ & & \\
\hline 201478 & $\begin{array}{c}\text { Graphic } \\
\text { Consultants } \\
\text { LTD. }\end{array}$ & $1972-0105$ & $\begin{array}{l}\text { Seeing } \\
\text { Canada }\end{array}$ & $\begin{array}{c}\text { The Playground of } \\
\text { a Continent }\end{array}$ & $1919 ?$ & cn & $\begin{array}{c}\text { Pathescope of } \\
\text { Canada }\end{array}$ & $\begin{array}{c}\text { Exhibits and } \\
\text { Publicity Bureau }\end{array}$ & & \\
\hline 201479 & $\begin{array}{l}\text { Graphic } \\
\text { Consultants } \\
\text { LTD. } \\
\end{array}$ & $1972-0105$ & $\begin{array}{l}\text { Seeing } \\
\text { Canada }\end{array}$ & $\begin{array}{c}\text { The Most } \\
\text { Picturesque Spot } \\
\text { in North America }\end{array}$ & 1918 & $\mathrm{cn}$ & $\begin{array}{c}\text { Pathescope of } \\
\text { Canada }\end{array}$ & $\begin{array}{c}\text { Exhibits and } \\
\text { Publicity Bureau }\end{array}$ & & \\
\hline 202749 & $\begin{array}{l}\text { Graphic } \\
\text { Consultants } \\
\text { LTD. } \\
\end{array}$ & $1972-0105$ & & China the Ancient & 1921 & $\mathrm{cn}$ & $\begin{array}{c}\text { Pathescope of } \\
\text { Canada }\end{array}$ & & & $\begin{array}{c}\text { Dick Bird } \\
\text { photographe } \\
\mathrm{r}\end{array}$ \\
\hline 205660 & $\begin{array}{c}\text { Graphic } \\
\text { Consultants } \\
\text { LTD. } \\
\end{array}$ & $1972-0105$ & & $\begin{array}{c}\text { Grape Growing in } \\
\text { Ontario }\end{array}$ & $1923 ?$ & $\mathrm{cn}$ & $\begin{array}{c}\text { Pathescope of } \\
\text { Canada }\end{array}$ & OMPB & & \\
\hline 207296 & $\begin{array}{l}\text { Graphic } \\
\text { Consultants } \\
\text { LTD. } \\
\end{array}$ & $1972-0105$ & & Charlotte Corday & 1909 & $\mathrm{fr}$ & Pathe Freres & & & \\
\hline 207297 & $\begin{array}{l}\text { Graphic } \\
\text { Consultants } \\
\text { LTD. } \\
\end{array}$ & $1972-0105$ & & $\begin{array}{l}\text { The Provincial } \\
\text { Plowing Match }\end{array}$ & 1917 & $\mathrm{cn}$ & $\begin{array}{l}\text { Pathescope of } \\
\text { Canada }\end{array}$ & $\begin{array}{c}\text { Ontario } \\
\text { Department of } \\
\text { Agriculture; } \\
\text { OMPB } \\
\end{array}$ & & \\
\hline 207298 & $\begin{array}{l}\text { Graphic } \\
\text { Consultants } \\
\text { LTD. } \\
\end{array}$ & $1972-0105$ & & $\begin{array}{c}\text { The Heavy Draft } \\
\text { Horse }\end{array}$ & $1919 ?$ & $\mathrm{cn}$ & $\begin{array}{l}\text { Pathescope of } \\
\text { Canada }\end{array}$ & OMPB & & \\
\hline 207299 & $\begin{array}{l}\text { Graphic } \\
\text { Consultants } \\
\text { LTD. } \\
\end{array}$ & $1972-0105$ & & Ontario & 1927 & $\mathrm{cn}$ & OMPB & & & \\
\hline 207300 & $\begin{array}{l}\text { Graphic } \\
\text { Consultants } \\
\text { LTD. } \\
\end{array}$ & $1972-0105$ & & $\begin{array}{c}\text { Ontario } \\
\text { Experimental } \\
\text { Farms } \\
\end{array}$ & $1922 ?$ & $\mathrm{cn}$ & $\begin{array}{l}\text { Pathescope of } \\
\text { Canada }\end{array}$ & OMPB & & \\
\hline 207301 & $\begin{array}{l}\text { Graphic } \\
\text { Consultants } \\
\text { LTD. } \\
\end{array}$ & $1972-0105$ & & Ontario Wool & 1917 & $\mathrm{cn}$ & $\begin{array}{l}\text { Pathescope of } \\
\text { Canada }\end{array}$ & OMPB & & \\
\hline 207302 & $\begin{array}{c}\text { Graphic } \\
\text { Consultants } \\
\text { LTD. }\end{array}$ & $1972-0105$ & & $\begin{array}{c}\text { Stumping in New } \\
\text { Ontario }\end{array}$ & $1920 / 1922$ & $\mathrm{cn}$ & $\begin{array}{l}\text { Pathescope of } \\
\text { Canada }\end{array}$ & OMPB & & \\
\hline 207303 & $\begin{array}{l}\text { Graphic } \\
\text { Consultants } \\
\text { LTD. } \\
\end{array}$ & $1972-0105$ & & $\begin{array}{c}\text { [Ladies Lawn } \\
\text { Bowling } \\
\text { Tournament] } \\
\end{array}$ & $1920 / 1930$ & $\mathrm{cn}$ & $\mathrm{N} / \mathrm{A}$ & $\mathrm{N} / \mathrm{A}$ & & \\
\hline 207304 & $\begin{array}{l}\text { Graphic } \\
\text { Consultants } \\
\text { LTD. } \\
\end{array}$ & $1972-0105$ & & $\begin{array}{c}\text { Students of } \\
\text { Kemptville } \\
\text { Agricultural } \\
\text { School at Work } \\
\text { and Play } \\
\end{array}$ & 1925 & $\mathrm{cn}$ & OMPB & & & \\
\hline 213577 & $\begin{array}{l}\text { Graphic } \\
\text { Consultants } \\
\text { LTD. }\end{array}$ & $1972-0105$ & & $\begin{array}{l}\text { English Cricketers } \\
\text { in Toronto }\end{array}$ & 1932/1933? & $\mathrm{cn}$ & OMPB & & & \\
\hline 213579 & $\begin{array}{c}\text { Graphic } \\
\text { Consultants } \\
\text { LTD. }\end{array}$ & $1972-0105$ & & $\begin{array}{l}\text { Early Market } \\
\text { Garden Crops }\end{array}$ & 1920/1922? & $\mathrm{cn}$ & $\begin{array}{l}\text { Pathescope of } \\
\text { Canada }\end{array}$ & OMPB & & \\
\hline
\end{tabular}




\begin{tabular}{|c|c|c|c|c|c|c|c|c|c|c|}
\hline 213580 & $\begin{array}{l}\text { Graphic } \\
\text { Consultants } \\
\text { LTD. } \\
\end{array}$ & $1972-0105$ & & $\begin{array}{c}\text { The Canadian Red } \\
\text { Cross } \\
\end{array}$ & 1925 & $\mathrm{cn}$ & OMPB & & & \\
\hline 216872 & $\begin{array}{c}\text { Graphic } \\
\text { Consultants } \\
\text { LTD. } \\
\end{array}$ & 1972-0105 & & $\begin{array}{l}\text { Bicycles We Have } \\
\text { Met : [incomplete] }\end{array}$ & $1917 ?$ & us & $\begin{array}{l}\text { Bray Studios } \\
\text { Inc. } \\
\end{array}$ & & OMPB & $\begin{array}{l}\text { Tinted } \\
\text { unknown } \\
\text { colour } \\
\end{array}$ \\
\hline 216874 & $\begin{array}{c}\text { Graphic } \\
\text { Consultants } \\
\text { LTD. } \\
\end{array}$ & 1972-0105 & & $\begin{array}{c}\text { Home Canning of } \\
\text { Vegetables }\end{array}$ & 1917? & cn & $\begin{array}{c}\text { Pathescope of } \\
\text { Canada }\end{array}$ & OMPB & & \\
\hline 216875 & $\begin{array}{l}\text { Graphic } \\
\text { Consultants } \\
\text { LTD. } \\
\end{array}$ & 1972-0105 & $\begin{array}{l}\text { Better } \\
\text { Babies } \\
\end{array}$ & $\begin{array}{c}\text { Table Manners in } \\
\text { the Nursery }\end{array}$ & N/A & us & $\begin{array}{c}\text { Paramount; } \\
\text { Women's Home } \\
\text { Companion } \\
\end{array}$ & & OMPB & $\begin{array}{l}\text { Tinted } \\
\text { unknown } \\
\text { colour } \\
\end{array}$ \\
\hline 216876 & $\begin{array}{c}\text { Graphic } \\
\text { Consultants } \\
\text { LTD. } \\
\end{array}$ & 1972-0105 & & $\begin{array}{c}\text { Spraying and } \\
\text { Dusting Fruit } \\
\text { Trees } \\
\end{array}$ & 1916/1919 & $\mathrm{cn}$ & $\begin{array}{c}\text { Pathescope of } \\
\text { Canada } \\
\end{array}$ & OMPB & & \\
\hline 218905 & $\begin{array}{c}\text { Graphic } \\
\text { Consultants } \\
\text { LTD. } \\
\end{array}$ & 1972-0105 & & $\begin{array}{c}\text { State Funeral of } \\
\text { the Late Right } \\
\text { Honourable Sir } \\
\text { Wilfrid Laurier } \\
\text { February 22nd } \\
\end{array}$ & 1919 & $\mathrm{cn}$ & $\begin{array}{c}\text { Pathescope of } \\
\text { Canada }\end{array}$ & & OMPB & \\
\hline 220749 & $\begin{array}{c}\text { Graphic } \\
\text { Consultants } \\
\text { LTD. }\end{array}$ & $1972-0105$ & & $\begin{array}{c}\text { Our Forest } \\
\text { Heritage }=\text { Notre } \\
\text { Heritage Forestier } \\
: \text { [incomplete] }\end{array}$ & 1926 & $\mathrm{cn}$ & OMPB & $\begin{array}{l}\text { Canadian } \\
\text { Forestry } \\
\text { Association }\end{array}$ & & \\
\hline 234369 & $\begin{array}{c}\text { Graphic } \\
\text { Consultants } \\
\text { LTD. } \\
\end{array}$ & 1972-0105 & & $\begin{array}{c}\text { In Action with our } \\
\text { Canadian Troops : } \\
\text { [excerpt] = [With } \\
\text { the } 5 \text { th Infantry } \\
\text { Battalion from } \\
\text { Saskatchewan] = } \\
\text { With the Canadian } \\
\text { Force } \\
\end{array}$ & 1916/1918? & $\mathrm{cn}$ & $\begin{array}{c}\text { British Topical } \\
\text { Committee for } \\
\text { War Films }\end{array}$ & War Office & & \\
\hline 234370 & $\begin{array}{l}\text { Graphic } \\
\text { Consultants } \\
\text { LTD. } \\
\end{array}$ & 1972-0105 & $\begin{array}{l}\text { Canadian } \\
\text { National } \\
\text { Pictorial } \\
\end{array}$ & $\begin{array}{c}\text { [Canada's National } \\
\text { Parks : } \\
\text { incomplete] } \\
\end{array}$ & 1919/1920? & $\mathrm{cn}$ & $\begin{array}{c}\text { Pathescope of } \\
\text { Canada } \\
\end{array}$ & & OMPB & \\
\hline 234371 & $\begin{array}{c}\text { Graphic } \\
\text { Consultants } \\
\text { LTD. } \\
\end{array}$ & $1972-0105$ & & $\begin{array}{c}\text { Forest Vigil }= \\
\text { Keeping the Forest } \\
\text { Vigil } \\
\end{array}$ & $1929 ?$ & $\mathrm{cn}$ & OMPB & & & \\
\hline 234373 & $\begin{array}{c}\text { Graphic } \\
\text { Consultants } \\
\text { LTD. } \\
\end{array}$ & 1972-0105 & & $\begin{array}{c}\text { The Years, the } \\
\text { Seasons and the } \\
\text { Days }\end{array}$ & $1922 ?$ & cn & $\begin{array}{c}\text { Filmcraft } \\
\text { Industries Ltd. }\end{array}$ & OMPB & OMPB & \\
\hline 234374 & $\begin{array}{c}\text { Graphic } \\
\text { Consultants } \\
\text { LTD. } \\
\end{array}$ & 1972-0105 & & $\begin{array}{c}\text { Oleomargarine : } \\
\text { How It is Made in } \\
\text { Canada }\end{array}$ & $1918 ?$ & $\mathrm{cn}$ & $\begin{array}{c}\text { Pathescope of } \\
\text { Canada }\end{array}$ & & & \\
\hline 234610 & $\begin{array}{c}\text { Graphic } \\
\text { Consultants } \\
\text { LTD. } \\
\end{array}$ & 1972-0105 & & $\begin{array}{l}\text { Canadian Soldiers } \\
\text { in the Making: } \\
\text { [incomplete] }\end{array}$ & 1915 & $\mathrm{cn}$ & $\begin{array}{c}\text { Pathescope of } \\
\text { Canada }\end{array}$ & & & \\
\hline 239484 & $\begin{array}{l}\text { Graphic } \\
\text { Consultants } \\
\text { LTD. } \\
\end{array}$ & 1972-0105 & & $\begin{array}{c}\text { British Empire } \\
\text { Games : Hamilton }\end{array}$ & 1930 & $\mathrm{cn}$ & OMPB & & & \\
\hline 243604 & $\begin{array}{c}\text { Graphic } \\
\text { Consultants } \\
\text { LTD. } \\
\end{array}$ & $1972-0105$ & & $\begin{array}{c}\text { The Annual } \\
\text { Plowing Match } \\
1924: \text { incomplete }\end{array}$ & 1924 & $\mathrm{cn}$ & OMPB & & & \\
\hline 243605 & $\begin{array}{c}\text { Graphic } \\
\text { Consultants } \\
\text { LTD. } \\
\end{array}$ & 1972-0105 & & $\begin{array}{c}\text { Ontario Provincial } \\
\text { Plowing Match } \\
1930 \\
\end{array}$ & 1930 & cn & OMPB & & & \\
\hline 243606 & $\begin{array}{c}\text { Graphic } \\
\text { Consultants } \\
\text { LTD. } \\
\end{array}$ & 1972-0105 & & $\begin{array}{l}\text { Ploughing with } \\
\text { Horse and Tractor } \\
\end{array}$ & 1919 & cn & $\begin{array}{c}\text { Pathescope of } \\
\text { Canada }\end{array}$ & OMPB & & \\
\hline 243607 & $\begin{array}{l}\text { Graphic } \\
\text { Consultants } \\
\text { LTD. } \\
\end{array}$ & 1972-0105 & & $\begin{array}{c}\text { Annual Provincial } \\
\text { Plowing Match }\end{array}$ & 1923 & $\mathrm{cn}$ & $\begin{array}{c}\text { Imperial Film } \\
\text { Company }\end{array}$ & OMPB & & \\
\hline 243608 & $\begin{array}{c}\text { Graphic } \\
\text { Consultants } \\
\text { LTD. } \\
\end{array}$ & 1972-0105 & & $\begin{array}{c}\text { Why Not Use a } \\
\text { Tractor? }\end{array}$ & 1919? & $\mathrm{cn}$ & $\begin{array}{c}\text { Pathescope of } \\
\text { Canada } \\
\end{array}$ & OMPB & & \\
\hline 243609 & $\begin{array}{c}\text { Graphic } \\
\text { Consultants } \\
\text { LTD. } \\
\end{array}$ & $1972-0105$ & & $\begin{array}{l}\text { Turning the } \\
\text { Furrow }\end{array}$ & 1920 & $\mathrm{cn}$ & $\begin{array}{c}\text { Filmcraft } \\
\text { Industries Ltd. }\end{array}$ & OMPB & & \\
\hline
\end{tabular}




\begin{tabular}{|c|c|c|c|c|c|c|c|c|c|c|}
\hline 243610 & $\begin{array}{l}\text { Graphic } \\
\text { Consultants } \\
\text { LTD. } \\
\end{array}$ & 1972-0105 & & The Great Lakes & $1922 ?$ & $\mathrm{cn}$ & $\begin{array}{c}\text { Filmcraft } \\
\text { Industries Ltd. }\end{array}$ & OMPB & & \\
\hline 243611 & $\begin{array}{c}\text { Graphic } \\
\text { Consultants } \\
\text { LTD. } \\
\end{array}$ & 1972-0105 & & $\begin{array}{c}\text { Land of } \\
\text { Enchantment }\end{array}$ & 1926 & $\mathrm{cn}$ & OMPB & & & \\
\hline 243612 & $\begin{array}{c}\text { Graphic } \\
\text { Consultants } \\
\text { LTD. } \\
\end{array}$ & 1972-0105 & & $\begin{array}{l}\text { Ontario's } \\
\text { Killarney } \\
\end{array}$ & $\begin{array}{c}1925-11- \\
01 / 1926-08- \\
23 \\
\end{array}$ & cn & OMPB & & & \\
\hline 243613 & $\begin{array}{l}\text { Graphic } \\
\text { Consultants } \\
\text { LTD. } \\
\end{array}$ & 1972-0105 & & A Sea Gull Tour & $1928 ?$ & $\mathrm{cn}$ & OMPB & & & \\
\hline 244221 & $\begin{array}{c}\text { Graphic } \\
\text { Consultants } \\
\text { LTD. } \\
\end{array}$ & 1972-0105 & & $\begin{array}{c}\text { The Ploughing } \\
\text { Match } 1922\end{array}$ & 1922 & $\mathrm{cn}$ & $\begin{array}{c}\text { Pathescope of } \\
\text { Canada }\end{array}$ & OMPB & & \\
\hline 244222 & $\begin{array}{c}\text { Graphic } \\
\text { Consultants } \\
\text { LTD. } \\
\end{array}$ & 1972-0105 & & $\begin{array}{c}\text { With the Plows at } \\
\text { Brockville: } \\
\text { [incomplete] }\end{array}$ & $\begin{array}{c}1925-11- \\
01 / 1926-08- \\
23 \\
\end{array}$ & $\mathrm{cn}$ & OMPB & & & \\
\hline 244223 & $\begin{array}{c}\text { Graphic } \\
\text { Consultants } \\
\text { LTD. } \\
\end{array}$ & 1972-0105 & & $\begin{array}{c}\text { Ploughman's } \\
\text { Progress } \\
\end{array}$ & $1929 ?$ & at & N/A & & & \\
\hline 244225 & $\begin{array}{c}\text { Graphic } \\
\text { Consultants } \\
\text { LTD. } \\
\end{array}$ & 1972-0105 & & In Old Quebec & 1921/1923? & $\mathrm{cn}$ & $\begin{array}{c}\text { Filmcraft } \\
\text { Industries Ltd. }\end{array}$ & & OMPB & \\
\hline 244226 & $\begin{array}{c}\text { Graphic } \\
\text { Consultants } \\
\text { LTD. } \\
\end{array}$ & 1972-0105 & $\begin{array}{l}\text { Seeing } \\
\text { Canada } \\
\end{array}$ & In Old Quebec & $1917 / 1927$ & $\mathrm{cn}$ & CGMPB & & & \\
\hline 244674 & $\begin{array}{c}\text { Graphic } \\
\text { Consultants } \\
\text { LTD. } \\
\end{array}$ & 1972-0105 & & The Colleen Bawn & 1911 & us & $\begin{array}{c}\text { Kalem Company } \\
\text { Inc. } \\
\end{array}$ & & $\begin{array}{c}\text { General Film } \\
\text { Company }\end{array}$ & $\begin{array}{l}1914 \text { re- } \\
\text { release } \\
\text { version } \\
\end{array}$ \\
\hline 248007 & $\begin{array}{c}\text { Graphic } \\
\text { Consultants } \\
\text { LTD. }\end{array}$ & $1972-0105$ & & $\begin{array}{c}\text { [Military Parade } \\
\text { and Gathering : } \\
\text { Aurora : } \\
\text { incomplete] }\end{array}$ & $1914 ?$ & $\mathrm{cn}$ & N/A & N/A & & \\
\hline 251680 & $\begin{array}{c}\text { Graphic } \\
\text { Consultants } \\
\text { LTD. } \\
\end{array}$ & 1972-0105 & & $\begin{array}{l}\text { With the Glasgow } \\
\text { Rangers in Ontario }\end{array}$ & 1930 & $\mathrm{cn}$ & OMPB & & & \\
\hline 254877 & $\begin{array}{c}\text { Graphic } \\
\text { Consultants } \\
\text { LTD. } \\
\end{array}$ & 1972-0105 & & $\begin{array}{c}\text { Corn for Feed and } \\
\text { Seed } \\
\end{array}$ & $1922 ?$ & cn & $\begin{array}{c}\text { Pathescope of } \\
\text { Canada }\end{array}$ & OMPB & & \\
\hline 254878 & $\begin{array}{c}\text { Graphic } \\
\text { Consultants } \\
\text { LTD. } \\
\end{array}$ & 1972-0105 & & $\begin{array}{c}\text { The Poultry Show } \\
: \text { [incomplete] }\end{array}$ & $1929 ?$ & cn & $\begin{array}{c}\text { Ontario } \\
\text { Government } \\
\text { Motion Picture } \\
\text { Bureau } \\
\end{array}$ & & & \\
\hline 254879 & $\begin{array}{l}\text { Graphic } \\
\text { Consultants } \\
\text { LTD. } \\
\end{array}$ & 1972-0105 & & $\begin{array}{l}\text { Boys' and Girls' } \\
\text { Livestock Clubs }\end{array}$ & $1922 ?$ & $\mathrm{cn}$ & $\begin{array}{c}\text { Pathescope of } \\
\text { Canada }\end{array}$ & OMPB & & \\
\hline 255362 & $\begin{array}{c}\text { Graphic } \\
\text { Consultants } \\
\text { LTD. } \\
\end{array}$ & 1972-0105 & & Sugar Beets & 1922 & $\mathrm{cn}$ & $\begin{array}{c}\text { Pathescope of } \\
\text { Canada }\end{array}$ & OMPB & & \\
\hline 257026 & $\begin{array}{c}\text { Graphic } \\
\text { Consultants } \\
\text { LTD. } \\
\end{array}$ & 1972-0105 & & $\begin{array}{c}\text { [Radium } \\
\text { Treatment for } \\
\text { Cancer : } \\
\text { incomplete] } \\
\end{array}$ & $\mathrm{N} / \mathrm{A}$ & $\mathrm{cn}$ & N/A & OMPB & & $\begin{array}{c}\text { no } \\
\text { description } \\
\end{array}$ \\
\hline 258089 & $\begin{array}{c}\text { Graphic } \\
\text { Consultants } \\
\text { LTD. } \\
\end{array}$ & 1972-0105 & $\begin{array}{c}\text { Canadian } \\
\text { War Records } \\
\end{array}$ & $\begin{array}{c}\text { [The March into } \\
\text { Cologne] : } \\
\text { [incomplete] }\end{array}$ & 1918 & cn & N/A & N/A & & \\
\hline 269951 & $\begin{array}{c}\text { Graphic } \\
\text { Consultants } \\
\text { LTD. } \\
\end{array}$ & 1972-0105 & & $\begin{array}{c}\text { Paris Through } \\
\text { History : First } \\
\text { Series }= \\
\text { [Historical Paris : } \\
\text { First Series] } \\
\end{array}$ & $1915 / 1925$ & $\mathrm{fr}$ & Pathe Freres & & & \\
\hline 269952 & $\begin{array}{c}\text { Graphic } \\
\text { Consultants } \\
\text { LTD. } \\
\end{array}$ & 1972-0105 & & $\begin{array}{c}\text { Historical Paris : } \\
\text { Second Series }= \\
{[\text { Paris Through }} \\
\text { History : Second } \\
\text { Series] } \\
\end{array}$ & $1915 / 1920$ & $\mathrm{fr}$ & Pathe Freres & & & \\
\hline
\end{tabular}




\begin{tabular}{|c|c|c|c|c|c|c|c|c|c|c|}
\hline 272109 & $\begin{array}{c}\text { Graphic } \\
\text { Consultants } \\
\text { LTD. } \\
\end{array}$ & $1972-0105$ & Nick Winter & $\begin{array}{c}\text { [Unlikely } \\
\text { Acquaintance: } \\
\text { Unidentified Nick } \\
\text { Winter] } \\
\end{array}$ & 1913 & $\mathrm{fr}$ & Pathe Freres & & & \\
\hline 272110 & $\begin{array}{l}\text { Graphic } \\
\text { Consultants } \\
\text { LTD. } \\
\end{array}$ & $1972-0105$ & & The Beggar Maid & 1921-09-25 & us & $\begin{array}{c}\text { Triart } \\
\text { Productions Inc. }\end{array}$ & & $\begin{array}{c}\text { W.W. } \\
\text { Hodkinson } \\
\text { Corporation }\end{array}$ & $\begin{array}{l}\text { Tinted } \\
\text { unknown } \\
\text { colour } \\
\end{array}$ \\
\hline 369868 & $\begin{array}{l}\text { Graphic } \\
\text { Consultants } \\
\text { LTD. } \\
\end{array}$ & 1972-0105 & & $\begin{array}{c}\text { Watch Your Step } \\
\text { on the Water }\end{array}$ & $1920 / 1925$ & $\mathrm{cn}$ & OMPB & & & \\
\hline 369880 & $\begin{array}{l}\text { Graphic } \\
\text { Consultants } \\
\text { LTD. } \\
\end{array}$ & $1972-0105$ & & $\begin{array}{l}\text { Stepping Along } \\
\text { with Santa Claus }\end{array}$ & $1920 / 1925$ & $\mathrm{cn}$ & OMPB & & & $\begin{array}{c}\text { Santa Claus } \\
\text { parade in } \\
\text { Toronto. } \\
\end{array}$ \\
\hline 276006 & $\begin{array}{l}\text { Graphic } \\
\text { Consultants } \\
\text { LTD. } \\
\end{array}$ & $1972-0105$ & & $\begin{array}{l}\text { The Young Painter } \\
: \text { [incomplete] }\end{array}$ & 1922-02-19 & us & $\begin{array}{c}\text { Triart } \\
\text { Productions Inc. }\end{array}$ & & $\begin{array}{c}\text { W.W. } \\
\text { Hodkinson } \\
\text { Corporation }\end{array}$ & $\begin{array}{c}\text { Tinted rose } \\
\text { colour }\end{array}$ \\
\hline 277695 & $\begin{array}{l}\text { Graphic } \\
\text { Consultants } \\
\text { LTD. } \\
\end{array}$ & $1972-0105$ & $\begin{array}{l}\text { Along the } \\
\text { Highways } \\
\text { and Byways } \\
\text { of England }\end{array}$ & $\begin{array}{l}\text { Old London On } \\
\text { The Thames }=\text { The } \\
\text { Heart of the } \\
\text { Empire }\end{array}$ & 1930/1931 & $\mathrm{cn}$ & OMPB & & & \\
\hline 277696 & $\begin{array}{c}\text { Graphic } \\
\text { Consultants } \\
\text { LTD. } \\
\end{array}$ & $1972-0105$ & $\begin{array}{l}\text { Along the } \\
\text { Highways } \\
\text { and Byways } \\
\text { of England }\end{array}$ & Cornwall & $1930 / 1931$ & $\mathrm{cn}$ & OMPB & & & \\
\hline 277697 & $\begin{array}{c}\text { Graphic } \\
\text { Consultants } \\
\text { LTD. } \\
\end{array}$ & $1972-0105$ & & $\begin{array}{c}\text { The Treasures of } \\
\text { Britain } \\
\end{array}$ & $1926 ?$ & $\mathrm{cn}$ & $\mathrm{N} / \mathrm{A}$ & $\begin{array}{c}\text { Cunard and } \\
\text { Anchor- } \\
\text { Donaldson Lines } \\
\end{array}$ & & \\
\hline 277699 & $\begin{array}{l}\text { Graphic } \\
\text { Consultants } \\
\text { LTD. } \\
\end{array}$ & $1972-0105$ & & $\begin{array}{l}\text { Westbound: } \\
\text { [incomplete] }\end{array}$ & $1926 ?$ & $\mathrm{cn}$ & $\mathrm{N} / \mathrm{A}$ & $\begin{array}{c}\text { Cunard and } \\
\text { Anchor- } \\
\text { Donaldson Lines } \\
\end{array}$ & & \\
\hline 278011 & $\begin{array}{l}\text { Graphic } \\
\text { Consultants } \\
\text { LTD. } \\
\end{array}$ & $1972-0105$ & & $\begin{array}{l}\text { Highlands of } \\
\text { Oregon }\end{array}$ & 1919 & us & $\begin{array}{c}\text { Bray } \\
\text { Productions Inc. }\end{array}$ & & OMPB & \\
\hline 278012 & $\begin{array}{c}\text { Graphic } \\
\text { Consultants } \\
\text { LTD. } \\
\end{array}$ & 1972-0105 & $\begin{array}{l}\text { Along the } \\
\text { Highways } \\
\text { and Byways } \\
\text { of England }\end{array}$ & Wessex & $1930 / 1931$ & $\mathrm{cn}$ & OMPB & & & \\
\hline 278013 & $\begin{array}{c}\text { Graphic } \\
\text { Consultants } \\
\text { LTD. } \\
\end{array}$ & $1972-0105$ & $\begin{array}{c}\text { Along the } \\
\text { Highways } \\
\text { and Byways } \\
\text { of England = } \\
\text { Pictures of } \\
\text { the Empire } \\
\text { Wales }\end{array}$ & $\begin{array}{c}\text { Wales }=\text { Among } \\
\text { the Eryri } \\
\text { Mountains of } \\
\text { North Wales } \\
\end{array}$ & 1930/1931 & $\mathrm{cn}$ & OMPB & & & \\
\hline 278163 & $\begin{array}{l}\text { Graphic } \\
\text { Consultants } \\
\text { LTD. } \\
\end{array}$ & $1972-0105$ & & $\begin{array}{c}\text { Fete of the Flags = } \\
\text { French Soldiers }\end{array}$ & $1917-07-14$ & N/A & Pathe Freres & & & \\
\hline 278172 & $\begin{array}{l}\text { Graphic } \\
\text { Consultants } \\
\text { LTD. } \\
\end{array}$ & $1972-0105$ & & $\begin{array}{c}\text { Blighty : } \\
\text { [incomplete] }\end{array}$ & 1927 & $\mathrm{uk}$ & $\begin{array}{c}\text { Piccadilly } \\
\text { Pictures Ltd. }\end{array}$ & & & \\
\hline 278203 & $\begin{array}{c}\text { Graphic } \\
\text { Consultants } \\
\text { LTD. }\end{array}$ & $1972-0105$ & & $\begin{array}{c}\text { Another Nick } \\
\text { Winter Exploit }= \\
\text { Nick Winter et le } \\
\text { Rapt de Mlle } \\
\text { Verner }\end{array}$ & 1911 & $\mathrm{fr}$ & Pathe Freres & & & \\
\hline 278204 & $\begin{array}{c}\text { Graphic } \\
\text { Consultants } \\
\text { LTD. }\end{array}$ & $1972-0105$ & & $\begin{array}{c}\text { Nick Winter } \\
\text { Again = Les } \\
\text { Russes de Nick } \\
\text { Winter }\end{array}$ & 1911 & $\mathrm{fr}$ & Pathe Freres & & & \\
\hline 278205 & $\begin{array}{c}\text { Graphic } \\
\text { Consultants } \\
\text { LTD. } \\
\end{array}$ & $1972-0105$ & & $\begin{array}{l}\text { Nick Winter the } \\
\text { Thief and the } \\
\text { Sonambulist }= \\
\text { Nick Winter la } \\
\text { voleuse et la } \\
\text { somnambule } \\
\end{array}$ & $11 / 29 / 11$ & $\mathrm{fr}$ & Pathe Freres & & & \\
\hline 278206 & $\begin{array}{c}\text { Graphic } \\
\text { Consultants } \\
\text { LTD. } \\
\end{array}$ & 1972-0105 & & $\begin{array}{l}\text { Nick Winter and } \\
\text { the Affair at the } \\
\text { Hotel Celebric = }\end{array}$ & 1911-10-24 & $\mathrm{fr}$ & Pathe Freres & & & \\
\hline
\end{tabular}




\begin{tabular}{|c|c|c|c|c|c|c|c|c|c|}
\hline & & & & $\begin{array}{c}\text { Nick Winter et } \\
\text { l'affaire du } \\
\text { Célébric Hôtel| } \\
1911 \\
\end{array}$ & & & & & \\
\hline 279807 & $\begin{array}{c}\text { Graphic } \\
\text { Consultants } \\
\text { LTD. } \\
\end{array}$ & 1972-0105 & & $\begin{array}{c}\text { The Land of } \\
\text { Rivers }\end{array}$ & $1923 ?$ & $\mathrm{cn}$ & $\begin{array}{c}\text { Filmcraft } \\
\text { Industries Ltd. }\end{array}$ & OMPB & \\
\hline 279809 & $\begin{array}{c}\text { Graphic } \\
\text { Consultants } \\
\text { LTD. }\end{array}$ & $1972-0105$ & & $\begin{array}{l}\text { Muskoka the } \\
\text { Playground }\end{array}$ & 1930 & $\mathrm{cn}$ & OMPB & & \\
\hline 279810 & $\begin{array}{c}\text { Graphic } \\
\text { Consultants } \\
\text { LTD. }\end{array}$ & 1972-0105 & & Presqu'ile & $\mathrm{N} / \mathrm{A}$ & $\mathrm{cn}$ & OMPB & & $\begin{array}{l}\text { Tinted } \\
\text { unknown } \\
\text { colour } \\
\end{array}$ \\
\hline 279811 & $\begin{array}{c}\text { Graphic } \\
\text { Consultants } \\
\text { LTD. }\end{array}$ & 1972-0105 & & River of Delight & $1929 ?$ & $\mathrm{cn}$ & OMPB & & \\
\hline 279812 & $\begin{array}{c}\text { Graphic } \\
\text { Consultants } \\
\text { LTD. }\end{array}$ & $1972-0105$ & & $\begin{array}{l}\text { Summer Resorts } \\
\text { in Ontario }\end{array}$ & 1919/1923? & $\mathrm{cn}$ & $\begin{array}{c}\text { Filmcraft } \\
\text { Industries Ltd. }\end{array}$ & OMPB & \\
\hline 279813 & $\begin{array}{c}\text { Graphic } \\
\text { Consultants } \\
\text { LTD. }\end{array}$ & 1972-0105 & & $\begin{array}{l}\text { Trent Valley } \\
\text { Waterways: } \\
\text { [incomplete] }\end{array}$ & 1922 & $\mathrm{cn}$ & OMPB & & \\
\hline 279814 & $\begin{array}{c}\text { Graphic } \\
\text { Consultants } \\
\text { LTD. }\end{array}$ & $1972-0105$ & & $\begin{array}{c}\text { When Phi Beta } \\
\text { Phi Visited } \\
\text { Ontario }\end{array}$ & 1925 & $\mathrm{cn}$ & OMPB & & \\
\hline 279815 & $\begin{array}{c}\text { Graphic } \\
\text { Consultants } \\
\text { LTD. }\end{array}$ & 1972-0105 & & $\begin{array}{c}\text { When Summer } \\
\text { Comes }\end{array}$ & 1922 & $\mathrm{cn}$ & $\begin{array}{c}\text { Filmcraft } \\
\text { Industries Ltd. }\end{array}$ & OMPB & \\
\hline 279816 & $\begin{array}{c}\text { Graphic } \\
\text { Consultants } \\
\text { LTD. }\end{array}$ & $1972-0105$ & $\begin{array}{l}\text { Seeing } \\
\text { Canada }\end{array}$ & $\begin{array}{c}\text { Where it is } \\
\text { Always Vacation } \\
\text { Time }\end{array}$ & 1920 & $\mathrm{cn}$ & $\begin{array}{c}\text { Pathescope of } \\
\text { Canada }\end{array}$ & $\begin{array}{c}\text { Exhibits and } \\
\text { Publicity Bureau }\end{array}$ & \\
\hline 279817 & $\begin{array}{c}\text { Graphic } \\
\text { Consultants } \\
\text { LTD. }\end{array}$ & $1972-0105$ & & The Handicap & 1920 & $\mathrm{cn}$ & $\begin{array}{c}\text { Pathescope of } \\
\text { Canada }\end{array}$ & OMPB & \\
\hline 279818 & $\begin{array}{c}\text { Graphic } \\
\text { Consultants } \\
\text { LTD. }\end{array}$ & $1972-0105$ & $\begin{array}{l}\text { Seeing } \\
\text { Canada }\end{array}$ & $\begin{array}{l}\text { The Harvest of the } \\
\text { Sugar Maple Tree }\end{array}$ & 1918 & $\mathrm{cn}$ & $\begin{array}{c}\text { Exhibits and } \\
\text { Publicity Bureau }\end{array}$ & & \\
\hline 279819 & $\begin{array}{c}\text { Graphic } \\
\text { Consultants } \\
\text { LTD. }\end{array}$ & $1972-0105$ & $\begin{array}{c}\text { Ontario } \\
\text { Agricultural } \\
\text { College }\end{array}$ & Number 72 , Oats & 1919 & $\mathrm{cn}$ & $\begin{array}{c}\text { Pathescope of } \\
\text { Canada }\end{array}$ & OMPB & $\begin{array}{c}\text { little } \\
\text { description } \\
\text { info } \\
\end{array}$ \\
\hline 279820 & $\begin{array}{c}\text { Graphic } \\
\text { Consultants } \\
\text { LTD. }\end{array}$ & $1972-0105$ & & $\begin{array}{c}\text { Old Trinity } \\
\text { College }\end{array}$ & 1925 & $\mathrm{cn}$ & OMPB & & \\
\hline 279821 & $\begin{array}{c}\text { Graphic } \\
\text { Consultants } \\
\text { LTD. }\end{array}$ & $1972-0105$ & & $\begin{array}{c}\text { Ontario School for } \\
\text { the Deaf }\end{array}$ & 1925 & $\mathrm{cn}$ & OMPB & & \\
\hline 280152 & $\begin{array}{c}\text { Graphic } \\
\text { Consultants } \\
\text { LTD. }\end{array}$ & $1972-0105$ & & $\begin{array}{c}\text { Nipigon Trails = } \\
\text { Sur Les Sentiers } \\
\text { du Nipigon }\end{array}$ & $1924 ?$ & $\mathrm{cn}$ & $\begin{array}{l}\text { Pathescope of } \\
\text { Canada }\end{array}$ & OMPB & \\
\hline 283925 & $\begin{array}{c}\text { Graphic } \\
\text { Consultants } \\
\text { LTD. }\end{array}$ & $1972-0105$ & & The Bacon Hog & $1919 ?$ & $\mathrm{cn}$ & $\begin{array}{l}\text { Pathescope of } \\
\text { Canada }\end{array}$ & OMPB & \\
\hline 284689 & $\begin{array}{c}\text { Graphic } \\
\text { Consultants } \\
\text { LTD. }\end{array}$ & $1972-0105$ & & $\begin{array}{c}\text { France's Famous } \\
\text { Gun The } 75\end{array}$ & N/A & $\mathrm{fr}$ & Pathe Freres & & \\
\hline 284690 & $\begin{array}{c}\text { Graphic } \\
\text { Consultants } \\
\text { LTD. }\end{array}$ & $1972-0105$ & & $\begin{array}{c}\text { With the Canadian } \\
\text { Force : } \\
\text { [incomplete] }\end{array}$ & 1917 & uk & $\begin{array}{c}\text { Topical Film } \\
\text { Company }\end{array}$ & $\begin{array}{c}\text { War Office } \\
\text { Cinema } \\
\text { Committee for } \\
\text { Canadian War } \\
\text { Records Office } \\
\end{array}$ & \\
\hline 293370 & $\begin{array}{c}\text { Graphic } \\
\text { Consultants } \\
\text { LTD. }\end{array}$ & $1972-0105$ & & $\begin{array}{c}\text { Fighting Winter } \\
\text { on the King's } \\
\text { Highways }\end{array}$ & $1929 / 1930 ?$ & $\mathrm{cn}$ & OMPB & & \\
\hline 301926 & $\begin{array}{c}\text { Graphic } \\
\text { Consultants } \\
\text { LTD. }\end{array}$ & $1972-0105$ & & $\begin{array}{l}\text { Bright Brighter } \\
\text { Brighton }\end{array}$ & 1930/1933? & $\mathrm{cn}$ & OMPB & & \\
\hline
\end{tabular}




\begin{tabular}{|c|c|c|c|c|c|c|c|c|c|}
\hline 301927 & $\begin{array}{c}\text { Graphic } \\
\text { Consultants } \\
\text { LTD. } \\
\end{array}$ & 1972-0105 & & North England & 1930/1933? & $\mathrm{cn}$ & $\begin{array}{l}\text { Ontario Motion } \\
\text { Picture Bureau; } \\
\text { Gaumont Mirror }\end{array}$ & $\begin{array}{c}\text { Cunard and } \\
\text { Anchor- } \\
\text { Donaldson Lines }\end{array}$ & $\begin{array}{c}\text { Hand tinted } \\
\text { grass and } \\
\text { trees }\end{array}$ \\
\hline 302170 & $\begin{array}{c}\text { Graphic } \\
\text { Consultants } \\
\text { LTD. } \\
\end{array}$ & $1972-0105$ & & $\begin{array}{l}\text { [Northumberland : } \\
\text { incomplete] }\end{array}$ & $1930 ?$ & $\mathrm{cn}$ & OMPB & & \\
\hline 302171 & $\begin{array}{c}\text { Graphic } \\
\text { Consultants } \\
\text { LTD. } \\
\end{array}$ & 1972-0105 & & $\begin{array}{l}\text { Nitrate of Soda } \\
\text { Industry }\end{array}$ & $1927 ?$ & $\mathrm{cn}$ & OMPB & & \\
\hline 302172 & $\begin{array}{c}\text { Graphic } \\
\text { Consultants } \\
\text { LTD. } \\
\end{array}$ & 1972-0105 & & $\begin{array}{c}\text { The Orphans } \\
\text { Abroad }\end{array}$ & 1925 & $\mathrm{cn}$ & OMPB & & \\
\hline 302173 & $\begin{array}{c}\text { Graphic } \\
\text { Consultants } \\
\text { LTD. } \\
\end{array}$ & $1972-0105$ & & $\begin{array}{c}\text { Overcoming Life's } \\
\text { Handicap }\end{array}$ & $1922 ?$ & $\mathrm{cn}$ & $\begin{array}{c}\text { Pathescope of } \\
\text { Canada }\end{array}$ & OMPB & $\begin{array}{l}\text { tinted } \\
\text { yellow }\end{array}$ \\
\hline 302174 & $\begin{array}{c}\text { Graphic } \\
\text { Consultants } \\
\text { LTD. } \\
\end{array}$ & 1972-0105 & & Our Heritage & $1931 ?$ & $\mathrm{cn}$ & OMPB & $\begin{array}{c}\text { Toronto Humane } \\
\text { Society }\end{array}$ & \\
\hline 302175 & $\begin{array}{c}\text { Graphic } \\
\text { Consultants } \\
\text { LTD. } \\
\end{array}$ & 1972-0105 & & $\begin{array}{c}\text { Parade of the } \\
\text { Originals }\end{array}$ & $1934 ?$ & $\mathrm{cn}$ & OMPB & & \\
\hline 302176 & $\begin{array}{c}\text { Graphic } \\
\text { Consultants } \\
\text { LTD. } \\
\end{array}$ & 1972-0105 & & $\begin{array}{l}\text { New Colours for } \\
\text { Simcoe Foresters }\end{array}$ & 1920/1929? & $\mathrm{cn}$ & OMPB & & \\
\hline 302177 & $\begin{array}{c}\text { Graphic } \\
\text { Consultants } \\
\text { LTD. } \\
\end{array}$ & $1972-0105$ & & $\begin{array}{l}\text { The Community } \\
\text { Canning Centre }\end{array}$ & 1917 & $\mathrm{cn}$ & $\begin{array}{c}\text { Pathescope of } \\
\text { Canada }\end{array}$ & OMPB & $\begin{array}{l}\text { tinted } \\
\text { unknown } \\
\text { colour } \\
\end{array}$ \\
\hline 302178 & $\begin{array}{c}\text { Graphic } \\
\text { Consultants } \\
\text { LTD. } \\
\end{array}$ & 1972-0105 & & Floating the Forest & $1929 ?$ & $\mathrm{cn}$ & OMPB & & \\
\hline 302180 & $\begin{array}{c}\text { Graphic } \\
\text { Consultants } \\
\text { LTD. }\end{array}$ & $1972-0105$ & & $\begin{array}{l}\text { Improving the } \\
\text { Light Horse in } \\
\text { Ontario }\end{array}$ & $1933 ?$ & $\mathrm{cn}$ & OMPB & & \\
\hline 302181 & $\begin{array}{c}\text { Graphic } \\
\text { Consultants } \\
\text { LTD. }\end{array}$ & $1972-0105$ & & $\begin{array}{l}\text { In Vulcan's } \\
\text { Workshop }\end{array}$ & $1922 ?$ & $\mathrm{cn}$ & $\begin{array}{c}\text { Filmcraft } \\
\text { Industries Ltd. }\end{array}$ & OMPB & $\begin{array}{l}\text { tinted and } \\
\text { tone section } \\
\text { in part one }\end{array}$ \\
\hline 302182 & $\begin{array}{c}\text { Graphic } \\
\text { Consultants } \\
\text { LTD. }\end{array}$ & $1972-0105$ & & $\begin{array}{c}\text { Light For Their } \\
\text { Darkness }\end{array}$ & $1928 ?$ & $\mathrm{cn}$ & OMPB & & $\begin{array}{c}\text { lack of } \\
\text { description }\end{array}$ \\
\hline 302183 & $\begin{array}{c}\text { Graphic } \\
\text { Consultants } \\
\text { LTD. } \\
\end{array}$ & 1972-0105 & & $\begin{array}{l}\text { Liquid Power: } \\
\text { [incomplete] }\end{array}$ & 1923 & $\mathrm{cn}$ & $\begin{array}{c}\text { Pathescope of } \\
\text { Canada }\end{array}$ & OMPB & \\
\hline 302184 & $\begin{array}{c}\text { Graphic } \\
\text { Consultants } \\
\text { LTD. } \\
\end{array}$ & 1972-0105 & & $\begin{array}{c}\text { Midsummer } \\
\text { Charms in Elgin's } \\
\text { Country Town }\end{array}$ & $1922 ?$ & $\mathrm{cn}$ & $\begin{array}{c}\text { Pathescope of } \\
\text { Canada }\end{array}$ & OMPB & $\begin{array}{c}\text { tinted } \\
\text { unknown } \\
\text { colour } \\
\end{array}$ \\
\hline 302185 & $\begin{array}{c}\text { Graphic } \\
\text { Consultants } \\
\text { LTD. } \\
\end{array}$ & $1972-0105$ & & $\begin{array}{l}\text { Scientists in the } \\
\text { Making }\end{array}$ & $1927 ?$ & $\mathrm{cn}$ & OMPB & & $\begin{array}{c}\text { lack of } \\
\text { description }\end{array}$ \\
\hline 302186 & $\begin{array}{c}\text { Graphic } \\
\text { Consultants } \\
\text { LTD. } \\
\end{array}$ & 1972-0105 & & $\begin{array}{c}\text { Sir Robert } \\
\text { Falconer : } \\
\text { [incomplete] } \\
\end{array}$ & 1931/1933? & $\mathrm{cn}$ & OMPB & & \\
\hline 302188 & $\begin{array}{c}\text { Graphic } \\
\text { Consultants } \\
\text { LTD. } \\
\end{array}$ & $1972-0105$ & & $\begin{array}{l}\text { Wild Life in } \\
\text { Toronto }\end{array}$ & 1933/1934? & $\mathrm{cn}$ & OMPB & & \\
\hline 302189 & $\begin{array}{c}\text { Graphic } \\
\text { Consultants } \\
\text { LTD. } \\
\end{array}$ & $1972-0105$ & $\begin{array}{c}\text { Ye Old Time } \\
\text { Country } \\
\text { Fayre } \\
\end{array}$ & $\begin{array}{l}\text { Part Two : } \\
\text { Beaverton }\end{array}$ & 1925 & cn & OMPB & & \\
\hline 302333 & $\begin{array}{l}\text { Graphic } \\
\text { Consultants } \\
\text { LTD. } \\
\end{array}$ & $1972-0105$ & $\begin{array}{l}\text { Highways } \\
\text { and Byways } \\
\text { of England } \\
\end{array}$ & $\begin{array}{c}\text { Seeing 'Merrie } \\
\text { England' = Seeing } \\
\text { Merrie England } \\
\text { with the Cunard } \\
\text { and Anchor- } \\
\text { Donaldson Lines } \\
\text { camera } \\
\end{array}$ & 1926/1928? & $\mathrm{cn}$ & OMPB & $\begin{array}{c}\text { Cunard and } \\
\text { Anchor- } \\
\text { Donaldson Lines } \\
\end{array}$ & \\
\hline 302337 & $\begin{array}{c}\text { Graphic } \\
\text { Consultants } \\
\text { LTD. } \\
\end{array}$ & $1972-0105$ & & $\begin{array}{c}\text { Bonnie Scotland : } \\
\text { [incomplete] }\end{array}$ & $1926 / 1928 ?$ & uk & N/A & $\begin{array}{c}\text { Cunard and } \\
\text { Anchor- } \\
\text { Donaldson Lines }\end{array}$ & \\
\hline
\end{tabular}




\begin{tabular}{|c|c|c|c|c|c|c|c|c|c|c|}
\hline 302349 & $\begin{array}{c}\text { Graphic } \\
\text { Consultants } \\
\text { LTD. } \\
\end{array}$ & 1972-0105 & $\begin{array}{c}\text { Something } \\
\text { About } \\
\text { Australia } \\
\end{array}$ & $\begin{array}{c}\text { The Conquest of } \\
\text { the Mallee }\end{array}$ & N/A & at & $\begin{array}{l}\text { Ontario Motion } \\
\text { Picture Bureau }\end{array}$ & $\begin{array}{c}\text { Government of } \\
\text { the } \\
\text { Commonwealth } \\
\text { of Australia }\end{array}$ & & \\
\hline 302351 & $\begin{array}{c}\text { Graphic } \\
\text { Consultants } \\
\text { LTD. } \\
\end{array}$ & 1972-0105 & & $\begin{array}{l}\text { The Control of } \\
\text { Twitch Grass }\end{array}$ & 1919? & $\mathrm{cn}$ & $\begin{array}{c}\text { Pathescope of } \\
\text { Canada }\end{array}$ & OMPB & & \\
\hline 302354 & $\begin{array}{c}\text { Graphic } \\
\text { Consultants } \\
\text { LTD. } \\
\end{array}$ & 1972-0105 & & $\begin{array}{l}\text { Rearing Chickens: } \\
\text { incomplete }\end{array}$ & 1919? & $\mathrm{cn}$ & $\begin{array}{c}\text { Pathescope of } \\
\text { Canada }\end{array}$ & OMPB & & \\
\hline 302369 & $\begin{array}{c}\text { Graphic } \\
\text { Consultants } \\
\text { LTD. } \\
\end{array}$ & 1972-0105 & & $\begin{array}{c}\text { Agricultural } \\
\text { Extension Work } \\
\text { with Rural Young } \\
\text { People in Ontario } \\
\end{array}$ & $1933 ?$ & $\mathrm{cn}$ & OMPB & & & \\
\hline 302375 & $\begin{array}{c}\text { Graphic } \\
\text { Consultants } \\
\text { LTD. }\end{array}$ & 1972-0105 & & Winds & 1920/1929? & us & $\begin{array}{c}\text { Scientific } \\
\text { Educational } \\
\text { Corporation } \\
\end{array}$ & & & $\begin{array}{l}\text { tinted } \\
\text { yellow }\end{array}$ \\
\hline 302377 & $\begin{array}{c}\text { Graphic } \\
\text { Consultants } \\
\text { LTD. }\end{array}$ & 1972-0105 & & Forest Folk & 1926 & $\mathrm{cn}$ & OMPB & & & $\begin{array}{l}\text { tinted } \\
\text { yellow }\end{array}$ \\
\hline 302378 & $\begin{array}{c}\text { Graphic } \\
\text { Consultants } \\
\text { LTD. }\end{array}$ & 1972-0105 & & $\begin{array}{c}\text { Canadian Bureau } \\
\text { for the } \\
\text { Advancement of } \\
\text { Music }= \\
\text { Advancement of } \\
\text { Music : Group } \\
\text { Instruction in } \\
\text { Piano } \\
\end{array}$ & 1932 & $\mathrm{cn}$ & OMPB & & & \\
\hline 302379 & $\begin{array}{c}\text { Graphic } \\
\text { Consultants } \\
\text { LTD. } \\
\end{array}$ & 1972-0105 & & Hope & 1922-07-16 & us & $\begin{array}{c}\text { Triart } \\
\text { Productions Inc. }\end{array}$ & & $\begin{array}{c}\text { W.W. } \\
\text { Hodkinson } \\
\text { Corporation }\end{array}$ & \\
\hline 302380 & $\begin{array}{c}\text { Graphic } \\
\text { Consultants } \\
\text { LTD. }\end{array}$ & 1972-0105 & & $\begin{array}{c}\text { The Handling of } \\
\text { Bees : } \\
\text { [incomplete] }\end{array}$ & $1919 ?$ & $\mathrm{cn}$ & $\begin{array}{c}\text { Pathescope of } \\
\text { Canada }\end{array}$ & OMPB & & \\
\hline 302381 & $\begin{array}{c}\text { Graphic } \\
\text { Consultants } \\
\text { LTD. }\end{array}$ & $1972-0105$ & & $\begin{array}{l}\text { Our Enemy the } \\
\text { Wasp }\end{array}$ & 1920/1924? & N/A & Pathe Freres & & & \\
\hline 302382 & $\begin{array}{c}\text { Graphic } \\
\text { Consultants } \\
\text { LTD. } \\
\end{array}$ & 1972-0105 & & $\begin{array}{l}\text { [A Fox Hunt : } \\
\text { incomplete] }\end{array}$ & N/A & $\mathrm{cn}$ & OMPB & & & \\
\hline 302383 & $\begin{array}{c}\text { Graphic } \\
\text { Consultants } \\
\text { LTD. } \\
\end{array}$ & 1972-0105 & & Tulip Land & N/A & $\mathrm{cn}$ & OMPB & & & $\begin{array}{c}\text { unknown } \\
\text { date and tint } \\
\text { colour }\end{array}$ \\
\hline 302384 & $\begin{array}{c}\text { Graphic } \\
\text { Consultants } \\
\text { LTD. }\end{array}$ & 1972-0105 & & Making Pig Iron & $1922 ?$ & $\mathrm{cn}$ & $\begin{array}{c}\text { Filmcraft } \\
\text { Industries Ltd. }\end{array}$ & OMPB & & \\
\hline 302385 & $\begin{array}{l}\text { Graphic } \\
\text { Consultants } \\
\text { LTD. }\end{array}$ & $1972-0105$ & $\begin{array}{l}\text { Canadian } \\
\text { National } \\
\text { Pictorial }\end{array}$ & $\begin{array}{c}\text { [Spring Crop of } \\
\text { Black and Silver } \\
\text { Fox Pups snakes } \\
\text { and Stanley Park]: } \\
\text { [incomplete] }\end{array}$ & $1919 / 1921$ & $\mathrm{cn}$ & $\begin{array}{l}\text { Pathescope of } \\
\text { Canada }\end{array}$ & & & \\
\hline 302386 & $\begin{array}{c}\text { Graphic } \\
\text { Consultants } \\
\text { LTD. } \\
\end{array}$ & 1972-0105 & & $\begin{array}{l}\text { Our Mechanical } \\
\text { Servant the } \\
\text { Elevator: } \\
\text { [incomplete] }\end{array}$ & $\mathrm{N} / \mathrm{A}$ & us & $\begin{array}{l}\text { Bray Studios } \\
\text { Inc. }\end{array}$ & & & \\
\hline 302388 & $\begin{array}{c}\text { Graphic } \\
\text { Consultants } \\
\text { LTD. } \\
\end{array}$ & 1972-0105 & & $\begin{array}{l}\text { Industries of the } \\
\text { West Indies }\end{array}$ & $1920 ?$ & us & Paramount-Bray & & & \\
\hline 302389 & $\begin{array}{c}\text { Graphic } \\
\text { Consultants } \\
\text { LTD. } \\
\end{array}$ & 1972-0105 & & $\begin{array}{c}\text { Our Picturesque } \\
\text { Neighbors }\end{array}$ & 1918 & us & Daypho-Bray & & & \\
\hline 302390 & $\begin{array}{l}\text { Graphic } \\
\text { Consultants } \\
\text { LTD. }\end{array}$ & $1972-0105$ & & $\begin{array}{l}\text { The Exhibit of the } \\
\text { Seed Branch : } \\
\text { Royal Winter Fair } \\
: \text { [incomplete] }\end{array}$ & N/A & $\mathrm{cn}$ & N/A & N/A & & \\
\hline 302391 & $\begin{array}{c}\text { Graphic } \\
\text { Consultants } \\
\text { LTD. }\end{array}$ & $1972-0105$ & & $\begin{array}{c}\text { [Radium } \\
\text { Production For } \\
\text { Cancer Treatment } \\
\text { : incomplete] }\end{array}$ & N/A & $\mathrm{cn}$ & N/A & N/A & & \\
\hline
\end{tabular}




\begin{tabular}{|c|c|c|c|c|c|c|c|c|c|c|}
\hline 302392 & $\begin{array}{l}\text { Graphic } \\
\text { Consultants } \\
\text { LTD. }\end{array}$ & $1972-0105$ & & $\begin{array}{c}\text { The European } \\
\text { Corn Borer }\end{array}$ & 1922 & $\mathrm{cn}$ & $\begin{array}{c}\text { Filmcraft } \\
\text { Industries Ltd. } \\
\text { OMPB }\end{array}$ & $\begin{array}{l}\text { Department of } \\
\text { Agriculture, } \\
\text { Entomological } \\
\text { Branch; OMPB }\end{array}$ & & \\
\hline 302403 & $\begin{array}{c}\text { Graphic } \\
\text { Consultants } \\
\text { LTD. }\end{array}$ & $1972-0105$ & & $\begin{array}{c}\text { The Educational } \\
\text { Playgrounds = } \\
\text { [Summer Camp } \\
\text { For British } \\
\text { Children : } \\
\text { incomplete] }\end{array}$ & 1922 & $\mathrm{cn}$ & $\begin{array}{c}\text { Filmcraft } \\
\text { Industries Ltd. }\end{array}$ & OMPB & & toned blue \\
\hline 302406 & $\begin{array}{c}\text { Graphic } \\
\text { Consultants } \\
\text { LTD. }\end{array}$ & $1972-0105$ & & $\begin{array}{l}\text { The Cycle of the } \\
\text { Silkworm : } \\
\text { [incomplete] }\end{array}$ & $1920 / 1929 ?$ & $\mathrm{cn}$ & $\begin{array}{c}\text { Pathescope of } \\
\text { Canada }\end{array}$ & & OMPB & \\
\hline 302408 & $\begin{array}{c}\text { Graphic } \\
\text { Consultants } \\
\text { LTD. }\end{array}$ & $1972-0105$ & $\begin{array}{c}\text { The Exploits } \\
\text { of Elaine }\end{array}$ & $\begin{array}{c}\text { Episode } 9, \text { The } \\
\text { Death Ray }\end{array}$ & 1915 & us & $\begin{array}{l}\text { Eclectic-Pathe } \\
\text { Exchange }\end{array}$ & & & \\
\hline 302409 & $\begin{array}{c}\text { Graphic } \\
\text { Consultants } \\
\text { LTD. } \\
\end{array}$ & $1972-0105$ & & Incubation & 1919? & $\mathrm{cn}$ & $\begin{array}{c}\text { Pathescope of } \\
\text { Canada }\end{array}$ & OMPB & & \\
\hline 302415 & $\begin{array}{c}\text { Graphic } \\
\text { Consultants } \\
\text { LTD. }\end{array}$ & $1972-0105$ & $\begin{array}{c}\text { Something } \\
\text { About } \\
\text { Australia } \\
\end{array}$ & Station Life & N/A & $\mathrm{N} / \mathrm{A}$ & $\begin{array}{c}\text { Government of } \\
\text { the } \\
\text { Commonwealth } \\
\text { of Australia }\end{array}$ & & & \\
\hline 302416 & $\begin{array}{c}\text { Graphic } \\
\text { Consultants } \\
\text { LTD. }\end{array}$ & 1972-0105 & & $\begin{array}{c}\text { [Aurora Baseball } \\
\text { Team in Action : } \\
\text { incomplete] }\end{array}$ & N/A & $\mathrm{cn}$ & N/A & N/A & & $\begin{array}{c}\text { no } \\
\text { discription } \\
\end{array}$ \\
\hline 302417 & $\begin{array}{c}\text { Graphic } \\
\text { Consultants } \\
\text { LTD. }\end{array}$ & $1972-0105$ & & $\begin{array}{c}\text { Australia : } \\
\text { [incomplete] }\end{array}$ & 1922 & at & $\begin{array}{c}\text { Scientific } \\
\text { Educational } \\
\text { Corporation }\end{array}$ & & & \\
\hline 302418 & $\begin{array}{c}\text { Graphic } \\
\text { Consultants } \\
\text { LTD. }\end{array}$ & $1972-0105$ & & $\begin{array}{l}\text { [The Opening of } \\
\text { the Welland Canal } \\
\text { : incomplete] }\end{array}$ & $1921 ?$ & $\mathrm{cn}$ & $\mathrm{N} / \mathrm{A}$ & N/A & & $\begin{array}{c}\text { no } \\
\text { discription }\end{array}$ \\
\hline 302420 & $\begin{array}{c}\text { Graphic } \\
\text { Consultants } \\
\text { LTD. }\end{array}$ & $1972-0105$ & $\begin{array}{l}\text { The Exploits } \\
\text { of Elaine }\end{array}$ & $\begin{array}{l}\text { Episode } 4 \text { The } \\
\text { Frozen Safe : } \\
\text { [incomplete] }\end{array}$ & 1915 & us & $\begin{array}{l}\text { Eclectic-Pathe } \\
\text { Exchange }\end{array}$ & & & \\
\hline 302996 & $\begin{array}{c}\text { Graphic } \\
\text { Consultants } \\
\text { LTD. } \\
\end{array}$ & $1972-0105$ & & $\begin{array}{c}\text { Curiosities of New } \\
\text { York } \\
\end{array}$ & 1917 & us & $\begin{array}{l}\text { Bray Studios } \\
\text { Inc. }\end{array}$ & & & $\begin{array}{c}\text { Tinted } \\
\text { unknow } \\
\text { colour }\end{array}$ \\
\hline 303053 & $\begin{array}{c}\text { Graphic } \\
\text { Consultants } \\
\text { LTD. }\end{array}$ & $1972-0105$ & & $\begin{array}{c}\text { Astronomical } \\
\text { Aspects }\end{array}$ & 1920/1923? & $\mathrm{cn}$ & $\begin{array}{c}\text { Filmcraft } \\
\text { Industries Ltd. }\end{array}$ & & & \\
\hline 305824 & $\begin{array}{c}\text { Graphic } \\
\text { Consultants } \\
\text { LTD. }\end{array}$ & $1972-0105$ & & $\begin{array}{c}\text { Between Love and } \\
\text { Duty }\end{array}$ & 1912 & $\mathrm{fr}$ & Pathe Freres & & & \\
\hline 306218 & $\begin{array}{c}\text { Graphic } \\
\text { Consultants } \\
\text { LTD. }\end{array}$ & $1972-0105$ & & $\begin{array}{l}\text { Iron and Steel } \\
\text { From the Ore Bed } \\
\text { to the Smelter }\end{array}$ & $1917 / 1922$ & $\mathrm{cn}$ & $\begin{array}{c}\text { Pathescope of } \\
\text { Canada }\end{array}$ & OMPB & & $\begin{array}{c}\text { tinted } \\
\text { unknown } \\
\text { colour }\end{array}$ \\
\hline 306219 & $\begin{array}{c}\text { Graphic } \\
\text { Consultants } \\
\text { LTD. }\end{array}$ & $1972-0105$ & & Smelter Fires & $1922 ?$ & $\mathrm{cn}$ & $\begin{array}{c}\text { Filmcraft } \\
\text { Industries Ltd. }\end{array}$ & OMPB & & $\begin{array}{l}\text { tinted } \\
\text { unknown } \\
\text { colour }\end{array}$ \\
\hline 306610 & $\begin{array}{c}\text { Graphic } \\
\text { Consultants } \\
\text { LTD. }\end{array}$ & $1972-0105$ & & $\begin{array}{l}\text { Resuscitation from } \\
\text { Drowning and } \\
\text { Electrical Shock }\end{array}$ & $1922 / 1923 ?$ & $\mathrm{cn}$ & $\begin{array}{c}\text { Filmcraft } \\
\text { Industries Ltd. }\end{array}$ & OMPB & & $\begin{array}{c}\text { tinted } \\
\text { yellow }\end{array}$ \\
\hline 306611 & $\begin{array}{c}\text { Graphic } \\
\text { Consultants } \\
\text { LTD. }\end{array}$ & $1972-0105$ & & $\begin{array}{c}\text { How to Save a } \\
\text { Life }\end{array}$ & $1929 ?$ & $\mathrm{cn}$ & OMPB & & & \\
\hline 306640 & $\begin{array}{c}\text { Graphic } \\
\text { Consultants } \\
\text { LTD. } \\
\end{array}$ & $1972-0105$ & & $\begin{array}{c}\text { Technical } \\
\text { Education in } \\
\text { Toronto : } \\
\text { [incomplete] } \\
\end{array}$ & 1931 & $\mathrm{cn}$ & OMPB & & & \\
\hline 308856 & $\begin{array}{c}\text { Graphic } \\
\text { Consultants } \\
\text { LTD. }\end{array}$ & $1972-0105$ & & $\begin{array}{l}\text { Swimming to } \\
\text { Fame and Fortune } \\
: \text { [incomplete] }\end{array}$ & 1927/1931? & $\mathrm{cn}$ & OMPB & & & \\
\hline 309302 & $\begin{array}{c}\text { Graphic } \\
\text { Consultants } \\
\text { LTD. }\end{array}$ & $1972-0105$ & & $\begin{array}{l}\text { The Victory Fêtes } \\
: \text { [incomplete] }\end{array}$ & 1919? & N/A & Pathe Freres & & & \\
\hline
\end{tabular}




\begin{tabular}{|c|c|c|c|c|c|c|c|c|}
\hline 310111 & $\begin{array}{l}\text { Graphic } \\
\text { Consultants } \\
\text { LTD. } \\
\end{array}$ & $1972-0105$ & $\begin{array}{l}\text { Our Work }=\text { The } \\
\text { Work of the } \\
\text { Y.W.C.A. in } \\
\text { Tokyo Japan } \\
\end{array}$ & $1923 / 1924 ?$ & ja & $\mathrm{N} / \mathrm{A}$ & N/A & \\
\hline 310799 & $\begin{array}{l}\text { Graphic } \\
\text { Consultants } \\
\text { LTD. } \\
\end{array}$ & $1972-0105$ & $\begin{array}{l}\text { The Ploughing } \\
\text { Match 1922: } \\
\text { [incomplete] }\end{array}$ & $1922 ?$ & $\mathrm{cn}$ & OMPB & & $\begin{array}{c}\text { Part two of } \\
\text { ISN: } 244221 \\
\end{array}$ \\
\hline 311418 & $\begin{array}{l}\text { Graphic } \\
\text { Consultants } \\
\text { LTD. } \\
\end{array}$ & $1972-0105$ & $\begin{array}{c}\text { When the Mast } \\
\text { Bends }\end{array}$ & $1927 ?$ & $\mathrm{cn}$ & OMPB & & $\begin{array}{c}\text { tinted } \\
\text { orange/gree } \\
\text { n/blue }\end{array}$ \\
\hline 313171 & $\begin{array}{l}\text { Graphic } \\
\text { Consultants } \\
\text { LTD. } \\
\end{array}$ & $1972-0105$ & $\begin{array}{c}\text { Canada's Diamond } \\
\text { Jubilee }\end{array}$ & 1927 & $\mathrm{cn}$ & OMPB & & \\
\hline 313688 & $\begin{array}{c}\text { Graphic } \\
\text { Consultants } \\
\text { LTD. }\end{array}$ & $1972-0105$ & Little Gray Doors & 1928 & $\mathrm{cn}$ & OMPB & & $\begin{array}{c}\text { Unusual } \\
\text { fiction film } \\
\text { produced by } \\
\text { OMPB }\end{array}$ \\
\hline 318506 & $\begin{array}{c}\text { Graphic } \\
\text { Consultants } \\
\text { LTD. }\end{array}$ & 1972-0105 & $\begin{array}{c}\text { Mobilization of } \\
\text { the 127th York } \\
\text { Rangers Overseas } \\
\text { Battalion: } \\
\text { [incomplete] }\end{array}$ & 1916 & $\mathrm{cn}$ & N/A & N/A & \\
\hline 318912 & $\begin{array}{c}\text { Graphic } \\
\text { Consultants } \\
\text { LTD. } \\
\end{array}$ & $1972-0105$ & City of Stratford & 1929 & $\mathrm{cn}$ & OMPB & & \\
\hline 319599 & $\begin{array}{c}\text { Graphic } \\
\text { Consultants } \\
\text { LTD. } \\
\end{array}$ & $1972-0105$ & $\begin{array}{c}\text { Dyak Village in } \\
\text { Borneo = The } \\
\text { Village of Dayak } \\
\text { in Borneo }\end{array}$ & 1920/1925? & N/A & Pathe Freres & & \\
\hline 319600 & $\begin{array}{l}\text { Graphic } \\
\text { Consultants } \\
\text { LTD. }\end{array}$ & $1972-0105$ & $\begin{array}{l}\text { [Dedicating a } \\
\text { Saskatchewan } \\
\text { Monument] }\end{array}$ & 1920/1929? & $\mathrm{cn}$ & $\mathrm{N} / \mathrm{A}$ & N/A & $\begin{array}{c}\text { no } \\
\text { description. } \\
\text { Tinted } \\
\text { unknown } \\
\text { colour }\end{array}$ \\
\hline 322492 & $\begin{array}{c}\text { Graphic } \\
\text { Consultants } \\
\text { LTD. }\end{array}$ & 1972-0105 & $\begin{array}{c}\text { Turkey Time in } \\
\text { Manitoulin : } \\
\text { Poultry in the } \\
\text { Land of the Maple } \\
\text { Leaf }\end{array}$ & $1917 / 1929$ & $\mathrm{cn}$ & CGMPB & & \\
\hline 323168 & $\begin{array}{l}\text { Graphic } \\
\text { Consultants } \\
\text { LTD. } \\
\end{array}$ & $1972-0105$ & $\begin{array}{l}\text { Prince Edward : } \\
\text { The Smallest } \\
\text { County in Ontario }\end{array}$ & $1917 / 1922$ & $\mathrm{cn}$ & $\begin{array}{c}\text { Filmcraft } \\
\text { Industries Ltd. }\end{array}$ & OMPB & \\
\hline 323179 & $\begin{array}{c}\text { Graphic } \\
\text { Consultants } \\
\text { LTD. }\end{array}$ & $1972-0105$ & $\begin{array}{l}\text { As the Year } \\
\text { Unfolds }\end{array}$ & 1928 & $\mathrm{cn}$ & OMPB & & \\
\hline 323269 & $\begin{array}{c}\text { Graphic } \\
\text { Consultants } \\
\text { LTD. } \\
\end{array}$ & $1972-0105$ & $\begin{array}{c}\text { The Australians in } \\
\text { Toronto }\end{array}$ & $\begin{array}{c}1932-06- \\
29 / 1932-07- \\
02\end{array}$ & $\mathrm{cn}$ & OMPB & & \\
\hline 323316 & $\begin{array}{l}\text { Graphic } \\
\text { Consultants } \\
\text { LTD. } \\
\end{array}$ & $1972-0105$ & $\begin{array}{c}\text { Art at the } \\
\text { Technical School }\end{array}$ & 1919 & $\mathrm{cn}$ & $\begin{array}{c}\text { Filmcraft } \\
\text { Industries Ltd. }\end{array}$ & ОМРВ & \\
\hline 323471 & $\begin{array}{c}\text { Graphic } \\
\text { Consultants } \\
\text { LTD. } \\
\end{array}$ & $1972-0105$ & Angling for Bass & 1929 & $\mathrm{cn}$ & OMPB & & \\
\hline 323529 & $\begin{array}{l}\text { Graphic } \\
\text { Consultants } \\
\text { LTD. } \\
\end{array}$ & $1972-0105$ & In Old Quebec & $1926 ?$ & $\mathrm{cn}$ & $\mathrm{N} / \mathrm{A}$ & $\mathrm{N} / \mathrm{A}$ & $\begin{array}{c}\text { tinted } \\
\text { orange }\end{array}$ \\
\hline 325192 & $\begin{array}{c}\text { Graphic } \\
\text { Consultants } \\
\text { LTD. }\end{array}$ & $1972-0105$ & $\begin{array}{c}\text { A Springtime } \\
\text { Harvest }\end{array}$ & & $\mathrm{cn}$ & OMPB & & $\begin{array}{l}\text { tinted } \\
\text { yellow }\end{array}$ \\
\hline 325273 & $\begin{array}{c}\text { Graphic } \\
\text { Consultants } \\
\text { LTD. } \\
\end{array}$ & $1972-0105$ & $\begin{array}{c}\text { Helium : } \\
\text { [incomplete] }\end{array}$ & $1923 ?$ & $\mathrm{cn}$ & $\begin{array}{c}\text { Filmcraft } \\
\text { Industries Ltd. }\end{array}$ & OMPB & \\
\hline 326139 & $\begin{array}{c}\text { Graphic } \\
\text { Consultants } \\
\text { LTD. } \\
\end{array}$ & $1972-0105$ & $\begin{array}{c}\text { Guernseys }= \\
\text { Guernseys On } \\
\text { Ontario Farms } \\
\text { And At The } \\
\text { C.N.E. }\end{array}$ & $1920 / 1923$ & $\mathrm{cn}$ & $\begin{array}{c}\text { Pathescope of } \\
\text { Canada }\end{array}$ & OMPB & $\begin{array}{l}\text { tinted } \\
\text { yellow }\end{array}$ \\
\hline
\end{tabular}




\begin{tabular}{|c|c|c|c|c|c|c|c|c|c|c|}
\hline 326428 & $\begin{array}{c}\text { Graphic } \\
\text { Consultants } \\
\text { LTD. } \\
\end{array}$ & $1972-0105$ & & $\begin{array}{l}\text { One Hour of Love } \\
: \text { [incomplete] }\end{array}$ & 1927 & us & $\begin{array}{c}\text { Tiffany } \\
\text { Productions Inc. }\end{array}$ & & OMPB & \\
\hline 328854 & $\begin{array}{l}\text { Graphic } \\
\text { Consultants } \\
\text { LTD. } \\
\end{array}$ & $1972-0105$ & & Cotton & 1927 & $\mathrm{cn}$ & $\begin{array}{c}\text { Associated } \\
\text { Screen News } \\
\text { Ltd. } \\
\end{array}$ & $\begin{array}{c}\text { Dominion } \\
\text { Textile } \\
\text { Company } \\
\text { Limited } \\
\end{array}$ & OMPB & \\
\hline 330215 & $\begin{array}{l}\text { Graphic } \\
\text { Consultants } \\
\text { LTD. } \\
\end{array}$ & $1972-0105$ & & $\begin{array}{c}\text { What's in a Name? } \\
: \text { [incomplete] }\end{array}$ & 1919 & $\mathrm{cn}$ & $\begin{array}{l}\text { Pathescope of } \\
\text { Canada }\end{array}$ & & & $\begin{array}{l}\text { listed in the } \\
1931 \text { OMPB } \\
\text { catalogue }\end{array}$ \\
\hline 334476 & $\begin{array}{l}\text { Graphic } \\
\text { Consultants } \\
\text { LTD. } \\
\end{array}$ & $1972-0105$ & & $\begin{array}{c}\text { That Model from } \\
\text { Paris } \\
\end{array}$ & 1926-10-15 & us & $\begin{array}{c}\text { Tiffany } \\
\text { Productions Inc. }\end{array}$ & & & \\
\hline 334522 & $\begin{array}{l}\text { Graphic } \\
\text { Consultants } \\
\text { LTD. } \\
\end{array}$ & $1972-0105$ & $\begin{array}{l}\text { Gem of the } \\
\text { Screen }\end{array}$ & $\begin{array}{l}\text { Peeps into Puzzle } \\
\text { Land } \\
\end{array}$ & $1924 ?$ & N/A & N/A & $\begin{array}{c}\text { Cranfield and } \\
\text { Clarke }\end{array}$ & OMPB & \\
\hline 336740 & $\begin{array}{l}\text { Graphic } \\
\text { Consultants } \\
\text { LTD. } \\
\end{array}$ & $1972-0105$ & & The Robson Trail & 1920/1930? & $\mathrm{cn}$ & $\begin{array}{c}\text { Exhibits and } \\
\text { Publicity Bureau }\end{array}$ & & & \\
\hline 336742 & $\begin{array}{l}\text { Graphic } \\
\text { Consultants } \\
\text { LTD. } \\
\end{array}$ & $1972-0105$ & & $\begin{array}{c}\text { The Athabasca } \\
\text { Trail } \\
\end{array}$ & $1920 / 1930 ?$ & $\mathrm{cn}$ & $\begin{array}{c}\text { Ontario } \\
\text { Government } \\
\text { Film } \\
\end{array}$ & & $\begin{array}{c}\text { Pathescope of } \\
\text { Canada }\end{array}$ & $\begin{array}{l}\text { tinted and } \\
\text { toned } \\
\text { unknown } \\
\text { colour }\end{array}$ \\
\hline 339100 & $\begin{array}{l}\text { Graphic } \\
\text { Consultants } \\
\text { LTD. } \\
\end{array}$ & $1972-0105$ & & $\begin{array}{l}\text { From the Manger } \\
\text { to the Cross or } \\
\text { Jesus of Nazareth }\end{array}$ & 1913 & us & $\begin{array}{c}\text { Kalem Company } \\
\text { Inc. }\end{array}$ & & & \\
\hline 340417 & $\begin{array}{l}\text { Graphic } \\
\text { Consultants } \\
\text { LTD. } \\
\end{array}$ & $1972-0105$ & $\begin{array}{l}\text { Seeing } \\
\text { Canada } \\
\end{array}$ & Gentlemen Cadets & 1920/1930? & $\mathrm{cn}$ & CGMPB & $\begin{array}{c}\text { Department of } \\
\text { Trade and } \\
\text { Commerce } \\
\end{array}$ & & \\
\hline 341246 & $\begin{array}{l}\text { Graphic } \\
\text { Consultants } \\
\text { LTD. } \\
\end{array}$ & $1972-0105$ & & $\begin{array}{c}\text { Mining Ontario } \\
\text { Gypsum = } \\
\text { Gypsum Mining }\end{array}$ & $1926 ?$ & $\mathrm{cn}$ & $\begin{array}{l}\text { Ontario Motion } \\
\text { Picture Bureau }\end{array}$ & & & \\
\hline 343486 & $\begin{array}{l}\text { Graphic } \\
\text { Consultants } \\
\text { LTD. } \\
\end{array}$ & $1972-0105$ & & $\begin{array}{c}\text { [Better Bulls : } \\
\text { incomplete] }\end{array}$ & 1924 & $\mathrm{cn}$ & $\begin{array}{c}\text { Saskatchewan } \\
\text { Government } \\
\text { Bureau of } \\
\text { Publications, } \\
\text { Motion Picture } \\
\text { Branch } \\
\end{array}$ & & & \\
\hline 347865 & $\begin{array}{l}\text { Graphic } \\
\text { Consultants } \\
\text { LTD. } \\
\end{array}$ & $1972-0105$ & $\begin{array}{l}\text { Seeing } \\
\text { Canada }\end{array}$ & Unblazed Trails & $1920 ?$ & $\mathrm{cn}$ & $\begin{array}{c}\text { Exhibits and } \\
\text { Publicity Bureau }\end{array}$ & & OMPB & $\begin{array}{c}\text { Tinted } \\
\text { unknown } \\
\text { colour } \\
\end{array}$ \\
\hline 356350 & $\begin{array}{l}\text { Graphic } \\
\text { Consultants } \\
\text { LTD. } \\
\end{array}$ & $1972-0105$ & $\begin{array}{l}\text { A Pat } \\
\text { Sullivan } \\
\text { Comic } \\
\end{array}$ & $\begin{array}{l}\text { Felix in } \\
\text { Hollywood: } \\
\text { [incomplete] }\end{array}$ & 1923 & us & $\mathrm{N} / \mathrm{A}$ & & & $\begin{array}{l}\text { Tinted } \\
\text { unknown } \\
\text { colour } \\
\end{array}$ \\
\hline 359661 & $\begin{array}{l}\text { Graphic } \\
\text { Consultants } \\
\text { LTD. } \\
\end{array}$ & $1972-0105$ & & Culling Hens & 1923 & $\mathrm{cn}$ & $\begin{array}{c}\text { Filmcraft } \\
\text { Industries Ltd. }\end{array}$ & OMPB & & \\
\hline 359792 & $\begin{array}{l}\text { Graphic } \\
\text { Consultants } \\
\text { LTD. } \\
\end{array}$ & $1972-0105$ & & $\begin{array}{l}\text { The New Era in } \\
\text { Canadian } \\
\text { Agriculture }\end{array}$ & 1928 & $\mathrm{cn}$ & N/A & Massey-Harris & & $\begin{array}{l}\text { title is made } \\
\text { up and } \\
\text { produciton } \\
\text { company } \\
\text { unknown } \\
\end{array}$ \\
\hline 370120 & $\begin{array}{l}\text { Graphic } \\
\text { Consultants } \\
\text { LTD. } \\
\end{array}$ & 1972-0105 & & $\begin{array}{l}\text { Making the Most } \\
\text { of Manure }\end{array}$ & 1917/1919? & $\mathrm{cn}$ & $\begin{array}{c}\text { Pathescope of } \\
\text { Canada }\end{array}$ & OMPB & & \\
\hline 364628 & $\begin{array}{l}\text { Graphic } \\
\text { Consultants } \\
\text { LTD. } \\
\end{array}$ & $1972-0105$ & $\begin{array}{l}\text { Canadian } \\
\text { National } \\
\text { Pictorial } \\
\end{array}$ & $\begin{array}{l}\text { Floating } \\
\text { Fortresses as } \\
\text { Royal Escort } \\
\end{array}$ & $\begin{array}{c}1920 \mid \\
1920 ? \\
\end{array}$ & cn & $\begin{array}{c}\text { Pathescope of } \\
\text { Canada }\end{array}$ & OMPB & & \\
\hline 365652 & $\begin{array}{l}\text { Graphic } \\
\text { Consultants } \\
\text { LTD. } \\
\end{array}$ & $1972-0105$ & & $\begin{array}{l}\text { Canadian National } \\
\text { Exhibition }= \\
\text { Scenes at the } \\
\text { World's Greatest } \\
\text { Annual Exposition } \\
\text { Canadian National } \\
\text { Exhibition } \\
\text { Toronto Part 1: } \\
\text { Incomplete } \\
\end{array}$ & 1915 & $\mathrm{cn}$ & $\begin{array}{c}\text { Pathescope of } \\
\text { Canada }\end{array}$ & & & \\
\hline
\end{tabular}




\begin{tabular}{|c|c|c|c|c|c|c|c|c|c|c|}
\hline 365698 & $\begin{array}{c}\text { Graphic } \\
\text { Consultants } \\
\text { LTD. }\end{array}$ & $1972-0105$ & $\begin{array}{c}\text { The World's } \\
\text { Greatest } \\
\text { Story }\end{array}$ & $\begin{array}{c}\text { Episode } 14= \\
\text { [World War One } \\
\text { Flyers] : } \\
\text { incomplete } \\
\end{array}$ & 1918/1919? & uk & $\begin{array}{l}\text { War Office } \\
\text { Official; New } \\
\text { Era Film }\end{array}$ & & $\begin{array}{c}\text { Ashley } \\
\text { Exclusive } \\
\text { Films } \\
\end{array}$ & \\
\hline 365826 & $\begin{array}{c}\text { Graphic } \\
\text { Consultants } \\
\text { LTD. } \\
\end{array}$ & $1972-0105$ & & $\begin{array}{l}\text { The Marketing of } \\
\text { Live Stock }\end{array}$ & 1931 & $\mathrm{cn}$ & OMPB & $\begin{array}{c}\text { Department of } \\
\text { Agriculture }\end{array}$ & & $\begin{array}{l}\text { description } \\
\text { is limited }\end{array}$ \\
\hline 368704 & $\begin{array}{c}\text { Graphic } \\
\text { Consultants } \\
\text { LTD. } \\
\end{array}$ & $1972-0105$ & & $\begin{array}{c}\text { The Parts of a Bee } \\
\text { Hive }\end{array}$ & $1920 ?$ & $\mathrm{cn}$ & OMPB & $\begin{array}{c}\text { Department of } \\
\text { Agriculture }\end{array}$ & & $\begin{array}{c}\text { description } \\
\text { is limited }\end{array}$ \\
\hline 369118 & $\begin{array}{c}\text { Graphic } \\
\text { Consultants } \\
\text { LTD. }\end{array}$ & $1972-0105$ & & $\begin{array}{l}\text { The Story of a } \\
\text { Cocoa Bean }\end{array}$ & 1915/1925? & $\mathrm{cn}$ & $\begin{array}{c}\text { Pathescope of } \\
\text { Canada }\end{array}$ & $\begin{array}{c}\text { Cowan } \\
\text { Company [?] }\end{array}$ & & \\
\hline 369901 & $\begin{array}{c}\text { Graphic } \\
\text { Consultants } \\
\text { LTD. } \\
\end{array}$ & $1972-0105$ & & $\begin{array}{c}\text { Commercial } \\
\text { Queen Rearing } \\
\end{array}$ & 1925 & $\mathrm{cn}$ & OMPB & & & \\
\hline 379552 & $\begin{array}{c}\text { Graphic } \\
\text { Consultants } \\
\text { LTD. }\end{array}$ & $1972-0105$ & & $\begin{array}{l}\text { Liquid Air and } \\
\text { Application of } \\
\text { Intense Cold }\end{array}$ & 1920/1925? & N/A & Pathe Freres & & OMPB & \\
\hline 379554 & $\begin{array}{c}\text { Graphic } \\
\text { Consultants } \\
\text { LTD. } \\
\end{array}$ & $1972-0105$ & & $\begin{array}{l}\text { Experiment With } \\
\text { Liquid Air }\end{array}$ & 1920/1925? & N/A & Pathe Freres & & OMPB & \\
\hline 372055 & $\begin{array}{c}\text { Graphic } \\
\text { Consultants } \\
\text { LTD. }\end{array}$ & $1972-0105$ & & $\begin{array}{c}\text { Origin of Trinidad } \\
\text { Chocolate }\end{array}$ & $1920 ?$ & $\mathrm{~N} / \mathrm{A}$ & $\begin{array}{l}\text { The Permanent } \\
\text { Exhibition } \\
\text { Committee; } \\
\text { Tucker Picture } \\
\text { Company; } \\
\text { Trinidad, British } \\
\text { West Indies } \\
\end{array}$ & & & \\
\hline 372104 & $\begin{array}{c}\text { Graphic } \\
\text { Consultants } \\
\text { LTD. }\end{array}$ & $1972-0105$ & & Mush! & 1925 & $\mathrm{cn}$ & OMPB & & & \\
\hline 372844 & $\begin{array}{c}\text { Graphic } \\
\text { Consultants } \\
\text { LTD. } \\
\end{array}$ & 1972-0105 & $\begin{array}{l}\text { Seeing } \\
\text { Canada }\end{array}$ & $\begin{array}{c}\text { A Mountaineering } \\
\text { Memory }\end{array}$ & 1919 & $\mathrm{cn}$ & $\begin{array}{c}\text { Exhibits and } \\
\text { Publicity Bureau }\end{array}$ & & & $\begin{array}{l}\text { tinted } \\
\text { unknown } \\
\text { colour; } \\
\text { english } \\
\text { subtitles } \\
\text { onlu } \\
\end{array}$ \\
\hline 372845 & $\begin{array}{c}\text { Graphic } \\
\text { Consultants } \\
\text { LTD. }\end{array}$ & $1972-0105$ & $\begin{array}{l}\text { Seeing } \\
\text { Canada }\end{array}$ & $\begin{array}{c}\text { A Mountaineering } \\
\text { Memory }\end{array}$ & 1919 & $\mathrm{cn}$ & $\begin{array}{c}\text { Exhibits and } \\
\text { Publicity Bureau }\end{array}$ & & & $\begin{array}{l}\text { French and } \\
\text { English } \\
\text { Subtitles } \\
\end{array}$ \\
\hline 372852 & $\begin{array}{c}\text { Graphic } \\
\text { Consultants } \\
\text { LTD. }\end{array}$ & $1972-0105$ & & $\begin{array}{l}\text { Grape Juice : } \\
\text { From Vine to } \\
\text { Bottle }\end{array}$ & $1920 ?$ & us & $\begin{array}{l}\text { Pathescope } \\
\text { USA? }\end{array}$ & & & $\begin{array}{l}\text { Industrial } \\
\text { film by } \\
\text { Welch's } \\
\text { Grape Juice } \\
\text { Company }\end{array}$ \\
\hline 372853 & $\begin{array}{c}\text { Graphic } \\
\text { Consultants } \\
\text { LTD. } \\
\end{array}$ & 1972-0105 & & $\begin{array}{l}\text { The Making of a } \\
\text { Cooking Range : } \\
\text { From Thought to } \\
\text { Finished Product }\end{array}$ & $1920 ?$ & $\mathrm{cn}$ & $\begin{array}{c}\text { Pathescope of } \\
\text { Canada } \\
\end{array}$ & & & \\
\hline 374395 & $\begin{array}{c}\text { Graphic } \\
\text { Consultants } \\
\text { LTD. }\end{array}$ & $1972-0105$ & & $\begin{array}{l}\text { At the Courts of } \\
\text { King Winter }\end{array}$ & $1926 ?$ & $\mathrm{cn}$ & OMPB & & & $\begin{array}{l}\text { tinted } \\
\text { unknown } \\
\text { colour } \\
\end{array}$ \\
\hline 374743 & $\begin{array}{c}\text { Graphic } \\
\text { Consultants } \\
\text { LTD. }\end{array}$ & $1972-0105$ & & $\begin{array}{c}\text { The Buffs in } \\
\text { Ontario }\end{array}$ & $1926 ?$ & $\mathrm{cn}$ & OMPB & & & \\
\hline 374745 & $\begin{array}{c}\text { Graphic } \\
\text { Consultants } \\
\text { LTD. } \\
\end{array}$ & $1972-0105$ & & $\begin{array}{l}\text { At the Courts of } \\
\text { King Winter }\end{array}$ & $1926 ?$ & $\mathrm{cn}$ & OMPB & & & \\
\hline 374763 & $\begin{array}{c}\text { Graphic } \\
\text { Consultants } \\
\text { LTD. }\end{array}$ & $1972-0105$ & & Farm Drainage & 1922/1925? & $\mathrm{cn}$ & $\begin{array}{c}\text { Filmcraft } \\
\text { Industries Ltd. }\end{array}$ & OMPB & & \\
\hline 374770 & $\begin{array}{c}\text { Graphic } \\
\text { Consultants } \\
\text { LTD. } \\
\end{array}$ & $1972-0105$ & & $\begin{array}{c}\text { The Way of the } \\
\text { Lord }\end{array}$ & 1925 & $\mathrm{uk}$ & $\begin{array}{c}\text { British } \\
\text { Instructional } \\
\text { Films Ltd. }\end{array}$ & & & \\
\hline 374768 & $\begin{array}{c}\text { Graphic } \\
\text { Consultants } \\
\text { LTD. }\end{array}$ & $1972-0105$ & & $\begin{array}{l}\text { The Romance of } \\
\text { the Nile }\end{array}$ & 1920/1930? & N/A & $\begin{array}{c}\text { Scientific } \\
\text { Educational } \\
\text { Corporation }\end{array}$ & & OMPB & \\
\hline
\end{tabular}




\begin{tabular}{|c|c|c|c|c|c|c|c|c|c|c|}
\hline 376588 & $\begin{array}{c}\text { Graphic } \\
\text { Consultants } \\
\text { LTD. }\end{array}$ & $1972-0105$ & $\begin{array}{l}\text { Seeing } \\
\text { Canada }\end{array}$ & $\begin{array}{c}\text { Jasper of the } \\
\text { Lakes }\end{array}$ & 1921 & $\mathrm{cn}$ & $\begin{array}{c}\text { Exhibits and } \\
\text { Publicity Bureau }\end{array}$ & $\begin{array}{l}\text { National Parks } \\
\text { of Canada, } \\
\text { Department of } \\
\text { the Interior }\end{array}$ & OMPB & $\begin{array}{c}\text { lack of } \\
\text { description } \\
\text { and tinted } \\
\text { unknown } \\
\text { colour } \\
\end{array}$ \\
\hline 377694 & $\begin{array}{c}\text { Graphic } \\
\text { Consultants } \\
\text { LTD. }\end{array}$ & $1972-0105$ & & $\begin{array}{c}\text { Niagara's Winter } \\
\text { Wizardry }\end{array}$ & $1922 ?$ & $\mathrm{cn}$ & $\begin{array}{c}\text { Filmcraft } \\
\text { Industries Ltd. }\end{array}$ & OMPB & & \\
\hline 378028 & $\begin{array}{c}\text { Graphic } \\
\text { Consultants } \\
\text { LTD. }\end{array}$ & $1972-0105$ & & $\begin{array}{c}\text { Nipigon } \\
\text { Grandeurs : } \\
\text { [incomplete] }\end{array}$ & $1920 ?$ & $\mathrm{cn}$ & OMPB & & & $\begin{array}{l}\text { tinted } \\
\text { unknown } \\
\text { colour } \\
\end{array}$ \\
\hline 378273 & $\begin{array}{c}\text { Graphic } \\
\text { Consultants } \\
\text { LTD. }\end{array}$ & $1972-0105$ & & $\begin{array}{l}\text { The Chateaux of } \\
\text { the Loire }\end{array}$ & $1920 ?$ & $\mathrm{fr}$ & Pathe Freres & & & \\
\hline 378619 & $\begin{array}{c}\text { Graphic } \\
\text { Consultants } \\
\text { LTD. }\end{array}$ & 1972-0105 & & Fish Ahoy! & $1929 ?$ & $\mathrm{cn}$ & OMPB & & OMPB & $\begin{array}{c}\text { lack of } \\
\text { description }\end{array}$ \\
\hline 378620 & $\begin{array}{c}\text { Graphic } \\
\text { Consultants } \\
\text { LTD. }\end{array}$ & $1972-0105$ & & Northern Ontario & $1922 ?$ & $\mathrm{cn}$ & $\begin{array}{c}\text { Filmcraft } \\
\text { Industries Ltd. }\end{array}$ & OMPB & & $\begin{array}{l}\text { tinted } \\
\text { unknown } \\
\text { colour. }\end{array}$ \\
\hline 378777 & $\begin{array}{c}\text { Graphic } \\
\text { Consultants } \\
\text { LTD. }\end{array}$ & $1972-0105$ & $\begin{array}{l}\text { Seeing } \\
\text { Canada }\end{array}$ & $\begin{array}{l}\text { The Prince of } \\
\text { Playgrounds = Le } \\
\text { Paradis du sport }\end{array}$ & $1919 ?$ & $\mathrm{cn}$ & $\begin{array}{c}\text { Exhibits and } \\
\text { Publicity Bureau }\end{array}$ & & & \\
\hline 378780 & $\begin{array}{c}\text { Graphic } \\
\text { Consultants } \\
\text { LTD. }\end{array}$ & 1972-0105 & & $\begin{array}{c}\text { Niagara's Winter } \\
\text { Wizardry }\end{array}$ & $1922 ?$ & $\mathrm{cn}$ & $\begin{array}{c}\text { Filmcraft } \\
\text { Industries Ltd. }\end{array}$ & OMPB & & \\
\hline 378790 & $\begin{array}{c}\text { Graphic } \\
\text { Consultants } \\
\text { LTD. }\end{array}$ & $1972-0105$ & & $\begin{array}{c}\text { The Making of a } \\
\text { King }\end{array}$ & $1925 ?$ & us & B.M. Powell Inc. & & OMPB & \\
\hline 378911 & $\begin{array}{c}\text { Graphic } \\
\text { Consultants } \\
\text { LTD. } \\
\end{array}$ & $1972-0105$ & & $\begin{array}{c}\text { In the City of } \\
\text { Flowers }\end{array}$ & 1926 & $\mathrm{cn}$ & $\begin{array}{c}\text { Ontario Motion } \\
\text { Picture Bureau }\end{array}$ & & & \\
\hline 378970 & $\begin{array}{c}\text { Graphic } \\
\text { Consultants } \\
\text { LTD. }\end{array}$ & 1972-0105 & & $\begin{array}{l}\text { The Treatment of } \\
\text { Grain for Smut }\end{array}$ & $1922 ?$ & $\mathrm{cn}$ & $\begin{array}{c}\text { Pathescope of } \\
\text { Canada }\end{array}$ & OMPB & & \\
\hline 379531 & $\begin{array}{c}\text { Graphic } \\
\text { Consultants } \\
\text { LTD. }\end{array}$ & $1972-0105$ & & $\begin{array}{c}\text { A } \\
\text { Cinematographic } \\
\text { Wonder : } \\
\text { Decomposition of } \\
\text { Ultra Rapid } \\
\text { Movement } \\
\end{array}$ & 1920/1928? & N/A & Pathe Freres & & & \\
\hline 379541 & $\begin{array}{c}\text { Graphic } \\
\text { Consultants } \\
\text { LTD. }\end{array}$ & $1972-0105$ & $\begin{array}{l}\text { Seeing } \\
\text { Canada }\end{array}$ & $\begin{array}{c}\text { The Queen of the } \\
\text { Coast }\end{array}$ & 1920 & $\mathrm{cn}$ & $\begin{array}{c}\text { Exhibits and } \\
\text { Publicity Bureau }\end{array}$ & & & \\
\hline 379547 & $\begin{array}{c}\text { Graphic } \\
\text { Consultants } \\
\text { LTD. }\end{array}$ & 1972-0105 & & $\begin{array}{c}\text { How to Select a } \\
\text { Good Beef Animal }\end{array}$ & $1919 ?$ & $\mathrm{cn}$ & $\begin{array}{c}\text { Pathescope of } \\
\text { Canada }\end{array}$ & OMPB & & $\begin{array}{c}\text { This title is } \\
\text { listed in the } \\
\text { OMPB } \\
\text { catalogues } \\
\text { for } \\
1922,1923,1 \\
927,1929,19 \\
31 \\
\end{array}$ \\
\hline 380186 & $\begin{array}{c}\text { Graphic } \\
\text { Consultants } \\
\text { LTD. } \\
\end{array}$ & $1972-0105$ & & $\begin{array}{c}\text { By Quinte's } \\
\text { Shores }\end{array}$ & $1922 ?$ & $\mathrm{cn}$ & $\begin{array}{c}\text { Filmcraft } \\
\text { Industries Ltd. }\end{array}$ & OMPB & & \\
\hline 380934 & $\begin{array}{c}\text { Graphic } \\
\text { Consultants } \\
\text { LTD. }\end{array}$ & $1972-0105$ & & $\begin{array}{l}\text { The Prince of } \\
\text { Wales in Canada }\end{array}$ & 1919 & $\mathrm{cn}$ & $\begin{array}{c}\text { Pathescope of } \\
\text { Canada }\end{array}$ & & ОМРB & \\
\hline 382070 & $\begin{array}{c}\text { Graphic } \\
\text { Consultants } \\
\text { LTD. }\end{array}$ & $1972-0105$ & $\begin{array}{l}\text { Canadian } \\
\text { National } \\
\text { Pictorial } \\
\end{array}$ & $\begin{array}{c}\text { [Canadian Grown } \\
\text { Foxes] Snakes and } \\
\text { Stanley Park Zoo } \\
: \text { [incomplete] }\end{array}$ & $1919 / 1921$ & $\mathrm{cn}$ & $\begin{array}{c}\text { Pathescope of } \\
\text { Canada }\end{array}$ & & & $\begin{array}{l}\text { Tinted } \\
\text { unknown } \\
\text { colour } \\
\end{array}$ \\
\hline 381239 & $\begin{array}{c}\text { Graphic } \\
\text { Consultants } \\
\text { LTD. }\end{array}$ & 1972-0105 & & $\begin{array}{c}\text { The Herring } \\
\text { Industry = British } \\
\text { Herring Industry }\end{array}$ & 1926/1933? & uk & $\begin{array}{c}\text { Empire } \\
\text { Marketing Board }\end{array}$ & & & \\
\hline 381441 & $\begin{array}{c}\text { Graphic } \\
\text { Consultants } \\
\text { LTD. }\end{array}$ & 1972-0105 & & $\begin{array}{l}\text { The Drive : } \\
\text { [incomplete] }\end{array}$ & 1925 & $\mathrm{cn}$ & N/A & OMPB & & \\
\hline
\end{tabular}




\begin{tabular}{|c|c|c|c|c|c|c|c|c|c|c|}
\hline 382876 & $\begin{array}{c}\text { Graphic } \\
\text { Consultants } \\
\text { LTD. } \\
\end{array}$ & 1972-0105 & & $\begin{array}{c}\text { Fighting the Sand } \\
\text { Dunes }\end{array}$ & 1922 & $\mathrm{cn}$ & $\begin{array}{c}\text { Filmcraft } \\
\text { Industries Ltd. }\end{array}$ & OMPB & & \\
\hline 382925 & $\begin{array}{c}\text { Graphic } \\
\text { Consultants } \\
\text { LTD. }\end{array}$ & 1972-0105 & & $\begin{array}{c}\text { Twixt Cup and } \\
\text { Lip }\end{array}$ & 1920/1929? & $\mathrm{cn}$ & N/A & & OMPB & $\begin{array}{c}\text { No } \\
\text { information } \\
\text { on } \\
\text { production } \\
\text { or sponsor } \\
\text { company }\end{array}$ \\
\hline 382989 & $\begin{array}{c}\text { Graphic } \\
\text { Consultants } \\
\text { LTD. }\end{array}$ & 1972-0105 & & $\begin{array}{c}\text { Let's Go Fishing : } \\
\text { [incomplete] }\end{array}$ & 1920/1929? & us & $\begin{array}{c}\text { Cranfield and } \\
\text { Clarke }\end{array}$ & & OMPB & \\
\hline 382990 & $\begin{array}{c}\text { Graphic } \\
\text { Consultants } \\
\text { LTD. } \\
\end{array}$ & 1972-0105 & & Lets Go! & 1920/1929? & $\mathrm{N} / \mathrm{A}$ & $\mathrm{N} / \mathrm{A}$ & & & $\begin{array}{c}\text { No } \\
\text { information } \\
\text { on } \\
\text { production } \\
\text { or sponsor } \\
\text { company } \\
\end{array}$ \\
\hline 383035 & $\begin{array}{c}\text { Graphic } \\
\text { Consultants } \\
\text { LTD. }\end{array}$ & 1972-0105 & & Time & 1920/1929? & N/A & $\begin{array}{c}\text { Scientific } \\
\text { Educational } \\
\text { Corporation } \\
\end{array}$ & & OMPB & Tinted \\
\hline 383109 & $\begin{array}{c}\text { Graphic } \\
\text { Consultants } \\
\text { LTD. }\end{array}$ & 1972-0105 & & $\begin{array}{c}\text { Zoo Who's Who : } \\
\text { [incomplete] }\end{array}$ & 1920/1929? & N/A & $\mathrm{N} / \mathrm{A}$ & & OMPB & \\
\hline 383154 & $\begin{array}{c}\text { Graphic } \\
\text { Consultants } \\
\text { LTD. }\end{array}$ & 1972-0105 & $\begin{array}{l}\text { Seeing } \\
\text { Canada }\end{array}$ & Our Wild Life & 1920/1921? & $\mathrm{cn}$ & $\begin{array}{c}\text { Exhibits and } \\
\text { Publicity Bureau }\end{array}$ & & & \\
\hline 383220 & $\begin{array}{c}\text { Graphic } \\
\text { Consultants } \\
\text { LTD. }\end{array}$ & 1972-0105 & & $\begin{array}{c}\text { Our Youth in } \\
\text { Training } \\
\end{array}$ & 1920/1921? & $\mathrm{cn}$ & $\begin{array}{c}\text { Filmcraft } \\
\text { Industries Ltd. }\end{array}$ & OMPB & & \\
\hline 383406 & $\begin{array}{c}\text { Graphic } \\
\text { Consultants } \\
\text { LTD. } \\
\end{array}$ & 1972-0105 & & $\begin{array}{l}\text { Monte Carlo and } \\
\text { Its Environs }\end{array}$ & 1920/1924? & $\mathrm{fr}$ & Pathe Freres & & & \\
\hline 383436 & $\begin{array}{c}\text { Graphic } \\
\text { Consultants } \\
\text { LTD. }\end{array}$ & $1972-0105$ & $\begin{array}{l}\text { Gem of the } \\
\text { Screen }\end{array}$ & $\begin{array}{c}\text { Should a Husband } \\
\text { Tell? } \\
\end{array}$ & $1920 / 1922 ?$ & $\mathrm{uk}$ & $\begin{array}{c}\text { Cranfield and } \\
\text { Clarke; Red Seal }\end{array}$ & & & \\
\hline 383437 & $\begin{array}{c}\text { Graphic } \\
\text { Consultants } \\
\text { LTD. }\end{array}$ & 1972-0105 & & Silos for the Farm & 1920/1921? & $\mathrm{cn}$ & $\begin{array}{c}\text { Pathescope of } \\
\text { Canada }\end{array}$ & OMPB & & $\begin{array}{l}\text { LAC record } \\
\text { missing } \\
\text { prdcty }\end{array}$ \\
\hline 383454 & $\begin{array}{c}\text { Graphic } \\
\text { Consultants } \\
\text { LTD. } \\
\end{array}$ & 1972-0105 & $\begin{array}{c}\text { Hepworth Q- } \\
\text { riosity by } \\
\text { "Q" }\end{array}$ & Let's Paint & 1924 & uk & $\mathrm{N} / \mathrm{A}$ & & OMPB & \\
\hline 383455 & $\begin{array}{c}\text { Graphic } \\
\text { Consultants } \\
\text { LTD. }\end{array}$ & 1972-0105 & & $\begin{array}{c}\text { Tiny's } \\
\text { Troublesome } \\
\text { Tooth } \\
\end{array}$ & 1925/1929? & $\mathrm{cn}$ & $\mathrm{N} / \mathrm{A}$ & & OMPB & $\begin{array}{l}\text { Animator: } \\
\text { Walter } \\
\text { Swaffield } \\
\end{array}$ \\
\hline 383473 & $\begin{array}{c}\text { Graphic } \\
\text { Consultants } \\
\text { LTD. }\end{array}$ & 1972-0105 & & $\begin{array}{l}\text { The Mill on the } \\
\text { Floss }\end{array}$ & 1915 & us & $\begin{array}{c}\text { Thanhouser Film } \\
\text { Corporation }\end{array}$ & & $\begin{array}{l}\text { Mutual Film } \\
\text { Corporation }\end{array}$ & \\
\hline 415738 & $\begin{array}{c}\text { Graphic } \\
\text { Consultants } \\
\text { LTD. }\end{array}$ & $1972-0105$ & & $\begin{array}{l}\text { Gathering Hemp } \\
\text { in Brittany }\end{array}$ & 1920/1924? & $\mathrm{fr}$ & Pathe Freres & & & \\
\hline 384688 & $\begin{array}{c}\text { Graphic } \\
\text { Consultants } \\
\text { LTD. }\end{array}$ & $1972-0105$ & & If Matches Struck & $1924 ?$ & us & $\begin{array}{c}\text { Cranfield and } \\
\text { Clarke ; Red } \\
\text { Seal Pictures } \\
\text { Corp. }\end{array}$ & & & $\begin{array}{c}\text { Tinted } \\
\text { unknow } \\
\text { colour }\end{array}$ \\
\hline 384907 & $\begin{array}{c}\text { Graphic } \\
\text { Consultants } \\
\text { LTD. } \\
\end{array}$ & $1972-0105$ & $\begin{array}{l}\text { Seeing } \\
\text { Canada } \\
\end{array}$ & Thoroughbreds & $1920 / 1922 ?$ & $\mathrm{cn}$ & $\begin{array}{c}\text { Exhibits and } \\
\text { Publicity Bureau }\end{array}$ & & $\begin{array}{l}\text { OMPB \& } \\
\text { CGMPB } \\
\end{array}$ & \\
\hline 384912 & $\begin{array}{c}\text { Graphic } \\
\text { Consultants } \\
\text { LTD. }\end{array}$ & 1972-0105 & $\begin{array}{l}\text { Gem of the } \\
\text { Screen }\end{array}$ & $\begin{array}{c}\text { Up the River With } \\
\text { Molly }\end{array}$ & 1920/1924? & $\mathrm{uk}$ & $\begin{array}{c}\text { Red Seal } \\
\text { Production Corp. }\end{array}$ & & $\begin{array}{c}\text { Cranfield and } \\
\text { Clarke }\end{array}$ & $\begin{array}{c}\text { Tinted } \\
\text { unknow } \\
\text { colour }\end{array}$ \\
\hline 384915 & $\begin{array}{c}\text { Graphic } \\
\text { Consultants } \\
\text { LTD. } \\
\end{array}$ & 1972-0105 & $\begin{array}{l}\text { Gem of the } \\
\text { Screen }\end{array}$ & $\begin{array}{c}\text { Do You } \\
\text { Remember? }\end{array}$ & 1922 & $\mathrm{uk}$ & $\begin{array}{c}\text { Red Seal } \\
\text { Pictures Corp. }\end{array}$ & & $\begin{array}{c}\text { Cranfield and } \\
\text { Clarke }\end{array}$ & $\begin{array}{c}\text { Tinted } \\
\text { unknow } \\
\text { colour }\end{array}$ \\
\hline 384956 & $\begin{array}{l}\text { Graphic } \\
\text { Consultants } \\
\text { LTD. }\end{array}$ & 1972-0105 & & $\begin{array}{l}\text { Ye Songs of } \\
\text { Yesterday : Stanza } \\
\text { Number One }\end{array}$ & 1920/1924? & N/A & $\begin{array}{c}\text { T.J. Ray } \\
\text { Production }\end{array}$ & & & \\
\hline
\end{tabular}




\begin{tabular}{|c|c|c|c|c|c|c|c|c|c|c|}
\hline 384980 & $\begin{array}{c}\text { Graphic } \\
\text { Consultants } \\
\text { LTD. }\end{array}$ & 1972-0105 & & $\begin{array}{l}\text { Ye Songs of } \\
\text { Yesterday : Stanza } \\
\text { Number Two }\end{array}$ & $1920 / 1924 ?$ & N/A & $\begin{array}{c}\text { T.J. Ray } \\
\text { Production }\end{array}$ & & & \\
\hline 385535 & $\begin{array}{l}\text { Graphic } \\
\text { Consultants } \\
\text { LTD. } \\
\end{array}$ & 1972-0105 & & $\begin{array}{c}\text { Corrective } \\
\text { Gymnastics }\end{array}$ & 1918 & us & $\begin{array}{c}\text { Daypho-Bray; } \\
\text { Bray Studios } \\
\text { Inc. } \\
\end{array}$ & & & $\begin{array}{c}\text { Tinted } \\
\text { unknown } \\
\text { colour } \\
\end{array}$ \\
\hline 385751 & $\begin{array}{l}\text { Graphic } \\
\text { Consultants } \\
\text { LTD. } \\
\end{array}$ & $1972-0105$ & & $\begin{array}{c}\text { Arts and Crafts in } \\
\text { India }\end{array}$ & 1920/1924? & $\mathrm{fr}$ & Pathe Freres & & & \\
\hline 415455 & $\begin{array}{l}\text { Graphic } \\
\text { Consultants } \\
\text { LTD. } \\
\end{array}$ & 1972-0105 & & $\begin{array}{c}\text { Modern } \\
\text { Metallurgy }\end{array}$ & 1920/1924? & $\mathrm{fr}$ & Pathe Freres & & & \\
\hline 415456 & $\begin{array}{c}\text { Graphic } \\
\text { Consultants } \\
\text { LTD. } \\
\end{array}$ & 1972-0105 & & $\begin{array}{c}\text { The Story of the } \\
\text { Typewriter }\end{array}$ & 1920/1924? & N/A & Pathe Freres & & OMPB & \\
\hline 415458 & $\begin{array}{l}\text { Graphic } \\
\text { Consultants } \\
\text { LTD. } \\
\end{array}$ & 1972-0105 & $\begin{array}{l}\text { Goldwyn- } \\
\text { Bray- } \\
\text { Pictograph } \\
\end{array}$ & $\begin{array}{c}\text { Speeding Up the } \\
\text { Play }\end{array}$ & 1920 & N/A & $\begin{array}{l}\text { Bray Picture } \\
\text { Corporation }\end{array}$ & & & $\begin{array}{c}\text { Tinted in } \\
\text { several } \\
\text { colours } \\
\end{array}$ \\
\hline 415459 & $\begin{array}{l}\text { Graphic } \\
\text { Consultants } \\
\text { LTD. } \\
\end{array}$ & $1972-0105$ & & [Tapioca] & 1920/1924? & N/A & Pathescope & & & \\
\hline 386083 & $\begin{array}{l}\text { Graphic } \\
\text { Consultants } \\
\text { LTD. } \\
\end{array}$ & 1972-0105 & & $\begin{array}{l}\text { Making Blister } \\
\text { Copper }\end{array}$ & $1922 ?$ & $\mathrm{cn}$ & $\begin{array}{c}\text { Filmcraft } \\
\text { Industries Ltd. }\end{array}$ & OMPB & OMPB & \\
\hline 386230 & $\begin{array}{l}\text { Graphic } \\
\text { Consultants } \\
\text { LTD. } \\
\end{array}$ & 1972-0105 & $\begin{array}{l}\text { Biological } \\
\text { Control of } \\
\text { Insect Pests }\end{array}$ & $\begin{array}{c}\text { Number } 1 \\
\text { Parasite of the } \\
\text { European Corn } \\
\text { Borer } \\
\end{array}$ & 1922 & $\mathrm{cn}$ & $\begin{array}{c}\text { Filmcraft } \\
\text { Industries Ltd. }\end{array}$ & $\begin{array}{l}\text { Department of } \\
\text { Agriculture, } \\
\text { Entomological } \\
\text { Branch; OMPB }\end{array}$ & & \\
\hline 415460 & $\begin{array}{c}\text { Graphic } \\
\text { Consultants } \\
\text { LTD. }\end{array}$ & 1972-0105 & & $\begin{array}{l}\text { Vintage in } \\
\text { Burgundy }\end{array}$ & $1920 / 1924 ?$ & $\mathrm{fr}$ & Pathe Freres & & & \\
\hline 388078 & $\begin{array}{l}\text { Graphic } \\
\text { Consultants } \\
\text { LTD. } \\
\end{array}$ & $1972-0105$ & & $\begin{array}{c}\text { Aberdeen Angus } \\
\text { Classes at the } \\
\text { Canadian National } \\
\text { Exhibition } \\
\text { Toronto : } \\
\text { [incomplete] } \\
\end{array}$ & $1922 ?$ & $\mathrm{cn}$ & $\begin{array}{c}\text { Pathescope of } \\
\text { Canada }\end{array}$ & OMPB & & \\
\hline 388079 & $\begin{array}{c}\text { Graphic } \\
\text { Consultants } \\
\text { LTD. } \\
\end{array}$ & 1972-0105 & & $\begin{array}{c}\text { Aberdeen Angus } \\
\text { on Ontario Farms }\end{array}$ & $1922 ?$ & $\mathrm{cn}$ & $\begin{array}{c}\text { Pathescope of } \\
\text { Canada }\end{array}$ & OMPB & & \\
\hline 421505 & $\begin{array}{l}\text { Graphic } \\
\text { Consultants } \\
\text { LTD. } \\
\end{array}$ & $1972-0105$ & & $\begin{array}{l}\text { Diary of a Rocky } \\
\text { Mountain Ranger }\end{array}$ & $1923 ?$ & $\mathrm{cn}$ & $\begin{array}{c}\text { Pathescope of } \\
\text { Canada }\end{array}$ & & & \\
\hline 389131 & $\begin{array}{l}\text { Graphic } \\
\text { Consultants } \\
\text { LTD. } \\
\end{array}$ & 1972-0105 & $\begin{array}{l}\text { Seeing } \\
\text { Canada } \\
\end{array}$ & A Bird City & $1919-10-27$ & $\mathrm{cn}$ & $\begin{array}{c}\text { Exhibits and } \\
\text { Publicity Bureau } \\
\end{array}$ & & OMPB & $\begin{array}{c}\text { Tinted } \\
\text { unknown } \\
\text { colour } \\
\end{array}$ \\
\hline 416402 & $\begin{array}{l}\text { Graphic } \\
\text { Consultants } \\
\text { LTD. } \\
\end{array}$ & 1972-0105 & & $\begin{array}{c}\text { Manufacture of } \\
\text { Pipes }\end{array}$ & 1916/1924? & $\mathrm{fr}$ & Pathe Freres & & & \\
\hline 416403 & $\begin{array}{c}\text { Graphic } \\
\text { Consultants } \\
\text { LTD. } \\
\end{array}$ & $1972-0105$ & & $\begin{array}{l}\text { Modern Breeds of } \\
\text { Beef and Dairy } \\
\text { Breeds for Milk } \\
\text { Production } \\
\end{array}$ & $1925 / 1930 ?$ & $\mathrm{cn}$ & OMPB & & & \\
\hline 389845 & $\begin{array}{l}\text { Graphic } \\
\text { Consultants } \\
\text { LTD. } \\
\end{array}$ & $1972-0105$ & & $\begin{array}{c}\text { The Carrier } \\
\text { Indians of British } \\
\text { Columbia } \\
\end{array}$ & $1923 / 1927$ & $\mathrm{cn}$ & $\begin{array}{c}\text { National } \\
\text { Museum of } \\
\text { Canada, } \\
\text { Department of } \\
\text { Mines } \\
\end{array}$ & & $\begin{array}{c}\text { National } \\
\text { Museum of } \\
\text { Canada }\end{array}$ & \\
\hline 389924 & $\begin{array}{l}\text { Graphic } \\
\text { Consultants } \\
\text { LTD. } \\
\end{array}$ & 1972-0105 & & $\begin{array}{c}\text { Herefords at } \\
\text { Canadian National } \\
\text { Exhibition } \\
\end{array}$ & 1920/1922? & $\mathrm{cn}$ & $\begin{array}{c}\text { Pathescope of } \\
\text { Canada }\end{array}$ & OMPB & & \\
\hline 390420 & $\begin{array}{l}\text { Graphic } \\
\text { Consultants } \\
\text { LTD. } \\
\end{array}$ & 1972-0105 & & [Cancer] & $1920 / 1925 ?$ & N/A & N/A & & & \\
\hline 391544 & $\begin{array}{c}\text { Graphic } \\
\text { Consultants } \\
\text { LTD. } \\
\end{array}$ & 1972-0105 & & $\begin{array}{l}\text { Wireless } \\
\text { Telephony }\end{array}$ & 1920 & us & $\begin{array}{l}\text { Bray Picture } \\
\text { Corporation }\end{array}$ & & & $\begin{array}{c}\text { Tinted } \\
\text { unknown } \\
\text { colour } \\
\end{array}$ \\
\hline
\end{tabular}




\begin{tabular}{|c|c|c|c|c|c|c|c|c|c|c|}
\hline 391612 & $\begin{array}{c}\text { Graphic } \\
\text { Consultants } \\
\text { LTD. }\end{array}$ & 1972-0105 & & $\begin{array}{c}\text { [Funeral of Paul } \\
\text { Déroulède] }\end{array}$ & $1914 ?$ & fr & Pathe Freres & & & \\
\hline 392028 & $\begin{array}{c}\text { Graphic } \\
\text { Consultants } \\
\text { LTD. } \\
\end{array}$ & 1972-0105 & & $\begin{array}{c}\text { [Radium } \\
\text { Treatment for } \\
\text { Cancer : } \\
\text { incomplete] }\end{array}$ & 1930/1933? & $\mathrm{cn}$ & N/A & OMPB & & \\
\hline 413497 & $\begin{array}{c}\text { Graphic } \\
\text { Consultants } \\
\text { LTD. } \\
\end{array}$ & 1972-0105 & & $\begin{array}{c}\text { Ayrshires on } \\
\text { Ontario Farms }\end{array}$ & $1922 ?$ & $\mathrm{cn}$ & $\begin{array}{c}\text { Pathescope of } \\
\text { Canada }\end{array}$ & OMPB & & $\begin{array}{l}\text { Tinted } \\
\text { Yellow } \\
\end{array}$ \\
\hline 413498 & $\begin{array}{c}\text { Graphic } \\
\text { Consultants } \\
\text { LTD. }\end{array}$ & 1972-0105 & & $\begin{array}{c}\text { The Bacon Hog } \\
\text { and the Wiltshire } \\
\text { Side }\end{array}$ & 1922 & $\mathrm{cn}$ & $\begin{array}{c}\text { Pathescope of } \\
\text { Canada }\end{array}$ & OMPB & & $\begin{array}{l}\text { Tinted } \\
\text { unknown } \\
\text { colour } \\
\end{array}$ \\
\hline 413499 & $\begin{array}{c}\text { Graphic } \\
\text { Consultants } \\
\text { LTD. } \\
\end{array}$ & 1972-0105 & $\begin{array}{c}\text { Ye Old Time } \\
\text { Country } \\
\text { Fayre } \\
\end{array}$ & Part One : Simcoe & 1925 & $\mathrm{cn}$ & OMPB & & & \\
\hline 413500 & $\begin{array}{c}\text { Graphic } \\
\text { Consultants } \\
\text { LTD. } \\
\end{array}$ & 1972-0105 & & $\begin{array}{l}\text { [Bow River and } \\
\text { Lake Louise : } \\
\text { incomplete] }\end{array}$ & 1920/1929? & cn & N/A & N/A & & $\begin{array}{c}\text { Tinted } \\
\text { yellow and } \\
\text { B\&W }\end{array}$ \\
\hline 413501 & $\begin{array}{c}\text { Graphic } \\
\text { Consultants } \\
\text { LTD. } \\
\end{array}$ & 1972-0105 & & $\begin{array}{c}\text { Breeds of Beef } \\
\text { Cattle No. } 53\end{array}$ & 1920/1929? & us & Hildebrand & & & \\
\hline 413502 & $\begin{array}{c}\text { Graphic } \\
\text { Consultants } \\
\text { LTD. } \\
\end{array}$ & $1972-0105$ & & $\begin{array}{l}\text { The Construction } \\
\text { of a Bituminous } \\
\text { Penetration Road }\end{array}$ & $1922 ?$ & $\mathrm{cn}$ & $\begin{array}{c}\text { Filmcraft } \\
\text { Industries Ltd. }\end{array}$ & OMPB & & Tinted Sepia \\
\hline 413503 & $\begin{array}{c}\text { Graphic } \\
\text { Consultants } \\
\text { LTD. } \\
\end{array}$ & 1972-0105 & & $\begin{array}{c}\text { The Conformation } \\
\text { of a Holstein } \\
\text { Dairy Cow }\end{array}$ & 1920/1922? & $\mathrm{cn}$ & $\begin{array}{c}\text { Pathescope of } \\
\text { Canada } \\
\end{array}$ & & & \\
\hline 413504 & $\begin{array}{c}\text { Graphic } \\
\text { Consultants } \\
\text { LTD. }\end{array}$ & 1972-0105 & & $\begin{array}{l}\text { Dedication of } \\
\text { Colours at Upper } \\
\text { Canada College }\end{array}$ & $1919 ?$ & $\mathrm{cn}$ & $\begin{array}{c}\text { Pathescope of } \\
\text { Canada }\end{array}$ & & & \\
\hline 413505 & $\begin{array}{c}\text { Graphic } \\
\text { Consultants } \\
\text { LTD. } \\
\end{array}$ & $1972-0105$ & & Early Tomatoes & 1920/1921? & $\mathrm{cn}$ & $\begin{array}{c}\text { Pathescope of } \\
\text { Canada }\end{array}$ & OMPB & & \\
\hline 413506 & $\begin{array}{c}\text { Graphic } \\
\text { Consultants } \\
\text { LTD. } \\
\end{array}$ & $1972-0105$ & & Export Steers & 1921 & cn & $\begin{array}{c}\text { Pathescope of } \\
\text { Canada } \\
\end{array}$ & OMPB & & $\begin{array}{l}\text { Tinted } \\
\text { unknown } \\
\text { colour } \\
\end{array}$ \\
\hline 413507 & $\begin{array}{c}\text { Graphic } \\
\text { Consultants } \\
\text { LTD. } \\
\end{array}$ & 1972-0105 & & $\begin{array}{l}\text { The Co-operative } \\
\text { Marketing of Eggs }\end{array}$ & 1920/1921? & cn & $\begin{array}{c}\text { Pathescope of } \\
\text { Canada }\end{array}$ & OMPB & OMPB & $\begin{array}{l}\text { Tinted } \\
\text { yellow } \\
\end{array}$ \\
\hline 413508 & $\begin{array}{c}\text { Graphic } \\
\text { Consultants } \\
\text { LTD. } \\
\end{array}$ & 1972-0105 & & $\begin{array}{c}\text { Forcing Lettuce in } \\
\text { the Greenhouse }\end{array}$ & 1920/1921? & cn & $\begin{array}{c}\text { Pathescope of } \\
\text { Canada } \\
\end{array}$ & OMPB & & \\
\hline 413509 & $\begin{array}{c}\text { Graphic } \\
\text { Consultants } \\
\text { LTD. } \\
\end{array}$ & 1972-0105 & & Forcing Rhubarb & 1920/1921? & $\mathrm{cn}$ & $\begin{array}{c}\text { Pathescope of } \\
\text { Canada }\end{array}$ & OMPB & & \\
\hline 413510 & $\begin{array}{c}\text { Graphic } \\
\text { Consultants } \\
\text { LTD. } \\
\end{array}$ & $1972-0105$ & & $\begin{array}{l}\text { Forcing Tomatoes } \\
\text { in the GreenHouse }\end{array}$ & 1920/1921? & $\mathrm{cn}$ & $\begin{array}{c}\text { Pathescope of } \\
\text { Canada } \\
\end{array}$ & $\begin{array}{c}\text { OMPB; } \\
\text { Department of } \\
\text { Agriculture } \\
\end{array}$ & & \\
\hline 413511 & $\begin{array}{c}\text { Graphic } \\
\text { Consultants } \\
\text { LTD. } \\
\end{array}$ & 1972-0105 & & $\begin{array}{l}\text { From Egg to } \\
\text { Cooking Pot } \\
\end{array}$ & 1920/1924? & N/A & Pathe Freres & & OMPB & \\
\hline 413512 & $\begin{array}{c}\text { Graphic } \\
\text { Consultants } \\
\text { LTD. } \\
\end{array}$ & $1972-0105$ & & $\begin{array}{l}\text { Growing Root and } \\
\text { Vegetable Seed } \\
\end{array}$ & 1920/1921? & $\mathrm{cn}$ & $\begin{array}{c}\text { Pathescope of } \\
\text { Canada } \\
\end{array}$ & OMPB & & \\
\hline 413513 & $\begin{array}{c}\text { Graphic } \\
\text { Consultants } \\
\text { LTD. }\end{array}$ & 1972-0105 & & $\begin{array}{c}\text { Guernseys in } \\
\text { Ontario Pastures : } \\
\text { [incomplete] }\end{array}$ & $1920 / 1922 ?$ & $\mathrm{cn}$ & $\begin{array}{c}\text { Filmcraft } \\
\text { Industries Ltd. }\end{array}$ & OMPB & & \\
\hline 413514 & $\begin{array}{c}\text { Graphic } \\
\text { Consultants } \\
\text { LTD. }\end{array}$ & 1972-0105 & & $\begin{array}{c}\text { Handy Farm } \\
\text { Devices }\end{array}$ & 1920/1921? & $\mathrm{cn}$ & $\begin{array}{c}\text { Pathescope of } \\
\text { Canada }\end{array}$ & OMPB & & \\
\hline 413515 & $\begin{array}{c}\text { Graphic } \\
\text { Consultants } \\
\text { LTD. }\end{array}$ & $1972-0105$ & & $\begin{array}{l}\text { Harvesting the } \\
\text { Earth's Crust : } \\
\text { Limestone }\end{array}$ & 1925 & $\mathrm{cn}$ & OMPB & & & $\begin{array}{c}\text { Tinted } \\
\text { unknown } \\
\text { colour; } \\
\text { Special print } \\
\text { with extra } \\
\text { intertitles } \\
\text { from other }\end{array}$ \\
\hline
\end{tabular}




\begin{tabular}{|c|c|c|c|c|c|c|c|c|}
\hline & & & & & & & & prints \\
\hline 413516 & $\begin{array}{c}\text { Graphic } \\
\text { Consultants } \\
\text { LTD. } \\
\end{array}$ & 1972-0105 & $\begin{array}{l}\text { Harvesting the } \\
\text { Earth's Crust: The } \\
\text { Quartzite Industry } \\
\text { in Ontario } \\
\end{array}$ & 1925 & $\mathrm{cn}$ & OMPB & & \\
\hline 413517 & $\begin{array}{c}\text { Graphic } \\
\text { Consultants } \\
\text { LTD. } \\
\end{array}$ & 1972-0105 & $\begin{array}{c}\text { Herefords on } \\
\text { Ontario Farms } \\
\end{array}$ & 1920/1921? & $\mathrm{cn}$ & $\begin{array}{c}\text { Pathescope of } \\
\text { Canada } \\
\end{array}$ & OMPB & \\
\hline 413518 & $\begin{array}{c}\text { Graphic } \\
\text { Consultants } \\
\text { LTD. } \\
\end{array}$ & 1972-0105 & $\begin{array}{l}\text { Holsteins at the } \\
\text { CNE Toronto }\end{array}$ & 1920/1921? & $\mathrm{cn}$ & $\begin{array}{c}\text { Pathescope of } \\
\text { Canada }\end{array}$ & OMPB & \\
\hline 413519 & $\begin{array}{c}\text { Graphic } \\
\text { Consultants } \\
\text { LTD. } \\
\end{array}$ & 1972-0105 & $\begin{array}{c}\text { Holsteins on } \\
\text { Ontario Farms } \\
\end{array}$ & 1920/1921? & $\mathrm{cn}$ & $\begin{array}{c}\text { Pathescope of } \\
\text { Canada } \\
\end{array}$ & OMPB & \\
\hline 413520 & $\begin{array}{c}\text { Graphic } \\
\text { Consultants } \\
\text { LTD. }\end{array}$ & 1972-0105 & $\begin{array}{l}\text { The Hotbed in the } \\
\text { Home Garden }\end{array}$ & 1920/1921? & $\mathrm{cn}$ & $\begin{array}{c}\text { Pathescope of } \\
\text { Canada } \\
\end{array}$ & OMPB & \\
\hline 413521 & $\begin{array}{c}\text { Graphic } \\
\text { Consultants } \\
\text { LTD. }\end{array}$ & 1972-0105 & $\begin{array}{c}\text { Indoor Growing of } \\
\text { Cucumbers }\end{array}$ & $1926 ?$ & $\mathrm{cn}$ & OMPB & & \\
\hline 413522 & $\begin{array}{c}\text { Graphic } \\
\text { Consultants } \\
\text { LTD. }\end{array}$ & 1972-0105 & $\begin{array}{l}\text { Common Insects } \\
\text { on the Farm : } \\
\text { [incomplete] }\end{array}$ & 1920/1921? & $\mathrm{cn}$ & $\begin{array}{c}\text { Pathescope of } \\
\text { Canada } \\
\end{array}$ & OMPB & \\
\hline 413523 & $\begin{array}{c}\text { Graphic } \\
\text { Consultants } \\
\text { LTD. } \\
\end{array}$ & 1972-0105 & $\begin{array}{c}\text { Jerseys at CNE : } \\
\text { incomplete }\end{array}$ & 1920/1921? & $\mathrm{cn}$ & $\begin{array}{c}\text { Pathescope of } \\
\text { Canada } \\
\end{array}$ & OMPB & \\
\hline 413524 & $\begin{array}{c}\text { Graphic } \\
\text { Consultants } \\
\text { LTD. }\end{array}$ & 1972-0105 & $\begin{array}{c}\text { Jerseys on Ontario } \\
\text { Farms } \\
\end{array}$ & 1920/1921? & $\mathrm{cn}$ & $\begin{array}{c}\text { Pathescope of } \\
\text { Canada } \\
\end{array}$ & OMPB & \\
\hline 413525 & $\begin{array}{c}\text { Graphic } \\
\text { Consultants } \\
\text { LTD. }\end{array}$ & 1972-0105 & $\begin{array}{c}\text { Killing and } \\
\text { Dressing Poultry }\end{array}$ & 1920/1921? & $\mathrm{cn}$ & $\begin{array}{c}\text { Pathescope of } \\
\text { Canada } \\
\end{array}$ & OMPB & \\
\hline 413526 & $\begin{array}{c}\text { Graphic } \\
\text { Consultants } \\
\text { LTD. } \\
\end{array}$ & 1972-0105 & $\begin{array}{c}\text { The Fourth } \\
\text { Wrigley Marathon } \\
\text { Swim for Ladies } \\
\text { CNE } 1929= \\
\text { Ladies' Swim at } \\
\text { CNE } \\
\end{array}$ & 1929 & $\mathrm{cn}$ & OMPB & & \\
\hline 413527 & $\begin{array}{c}\text { Graphic } \\
\text { Consultants } \\
\text { LTD. } \\
\end{array}$ & 1972-0105 & $\begin{array}{c}\text { Livestock at the } \\
\text { CNE }\end{array}$ & 1920/1921? & $\mathrm{cn}$ & $\begin{array}{c}\text { Filmcraft } \\
\text { Industries Ltd. }\end{array}$ & OMPB & $\begin{array}{c}\text { Tinted } \\
\text { unknown } \\
\text { colour } \\
\end{array}$ \\
\hline 413528 & $\begin{array}{c}\text { Graphic } \\
\text { Consultants } \\
\text { LTD. } \\
\end{array}$ & 1972-0105 & $\begin{array}{c}\text { The Manufacture } \\
\text { of Woollen Cloth } \\
=\text { The } \\
\text { Manufacture of } \\
\text { Woollens }\end{array}$ & 1920/1922? & $\mathrm{cn}$ & $\begin{array}{c}\text { Pathescope of } \\
\text { Canada } \\
\end{array}$ & OMPB & $\begin{array}{l}\text { Tinted } \\
\text { yellow }\end{array}$ \\
\hline 413529 & $\begin{array}{c}\text { Graphic } \\
\text { Consultants } \\
\text { LTD. }\end{array}$ & 1972-0105 & $\begin{array}{l}\text { Milling Ontario's } \\
\text { Grain }\end{array}$ & $1922 ?$ & $\mathrm{cn}$ & $\begin{array}{c}\text { Pathescope of } \\
\text { Canada } \\
\end{array}$ & OMPB & \\
\hline 413531 & $\begin{array}{c}\text { Graphic } \\
\text { Consultants } \\
\text { LTD. } \\
\end{array}$ & 1972-0105 & $\begin{array}{c}\text { Onion Growing in } \\
\text { Ontario } \\
\end{array}$ & 1917/1918? & $\mathrm{cn}$ & $\begin{array}{c}\text { Pathescope of } \\
\text { Canada } \\
\end{array}$ & $\begin{array}{c}\text { Department of } \\
\text { Agriculture }\end{array}$ & \\
\hline 413532 & $\begin{array}{c}\text { Graphic } \\
\text { Consultants } \\
\text { LTD. }\end{array}$ & 1972-0105 & $\begin{array}{c}\text { Ontario Cattle at } \\
\text { Syracuse New } \\
\text { York } \\
\end{array}$ & 1923 & $\mathrm{cn}$ & $\begin{array}{c}\text { Pathescope of } \\
\text { Canada } \\
\end{array}$ & OMPB & $\begin{array}{l}\text { Tinted } \\
\text { yellow }\end{array}$ \\
\hline 413534 & $\begin{array}{c}\text { Graphic } \\
\text { Consultants } \\
\text { LTD. }\end{array}$ & 1972-0105 & School Gardens & 1920/1921? & $\mathrm{cn}$ & $\begin{array}{c}\text { Pathescope of } \\
\text { Canada } \\
\end{array}$ & OMPB & $\begin{array}{l}\text { Tinted } \\
\text { yellow }\end{array}$ \\
\hline 413535 & $\begin{array}{c}\text { Graphic } \\
\text { Consultants } \\
\text { LTD. } \\
\end{array}$ & 1972-0105 & $\begin{array}{c}\text { What's Under the } \\
\text { Fleece }\end{array}$ & 1917/1918? & $\mathrm{cn}$ & $\begin{array}{c}\text { Pathescope of } \\
\text { Canada }\end{array}$ & OMPB & \\
\hline 413536 & $\begin{array}{c}\text { Graphic } \\
\text { Consultants } \\
\text { LTD. } \\
\end{array}$ & 1972-0105 & $\begin{array}{l}\text { When Santa Claus } \\
\text { Comes to Toronto }\end{array}$ & 1929 & $\mathrm{cn}$ & OMPB & & \\
\hline
\end{tabular}




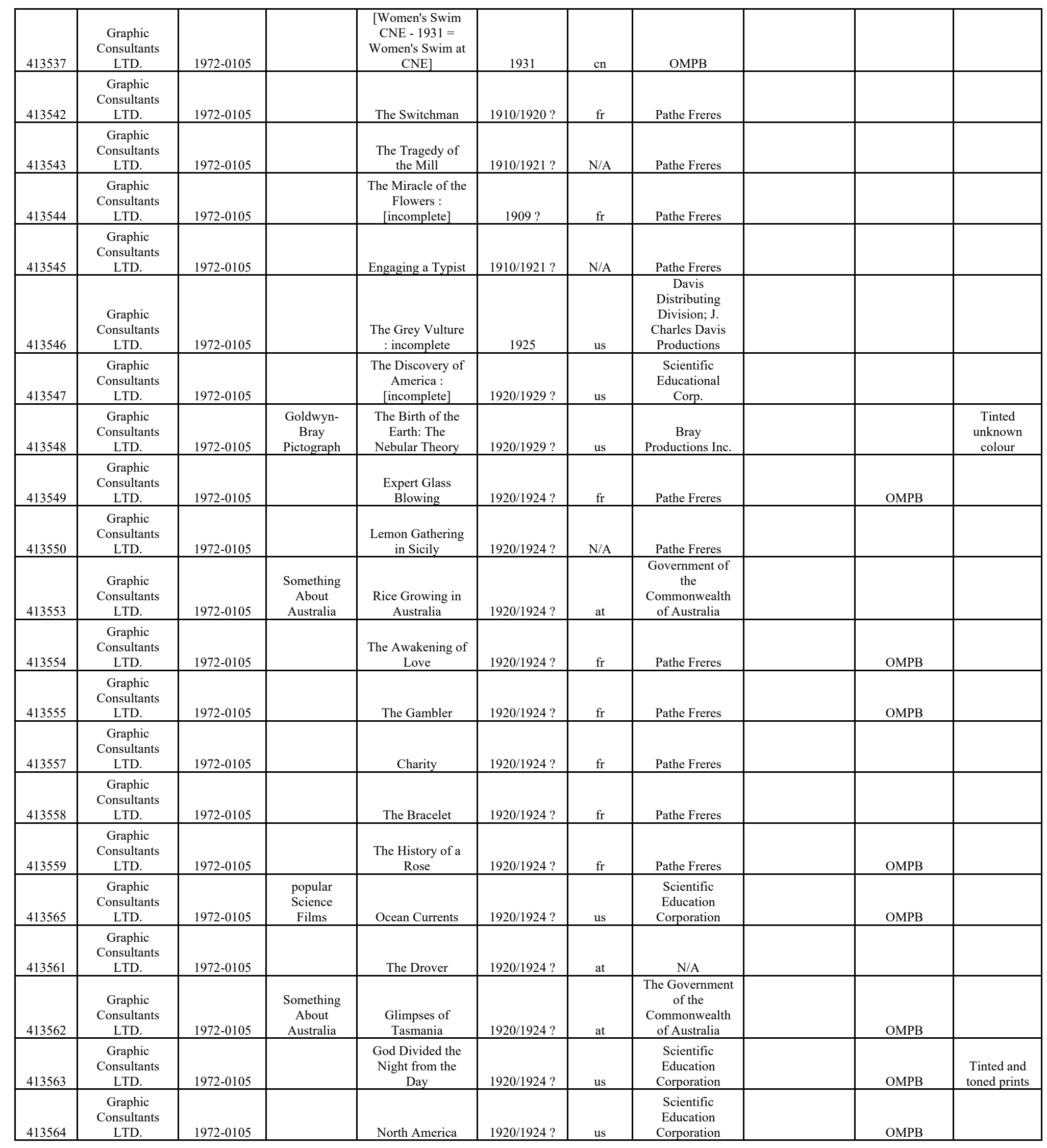




\begin{tabular}{|c|c|c|c|c|c|c|c|c|c|c|}
\hline 413566 & $\begin{array}{l}\text { Graphic } \\
\text { Consultants } \\
\text { LTD. } \\
\end{array}$ & $1972-0105$ & & The Gulf Stream & 1920/1924？ & us & $\begin{array}{l}\text { Scientific } \\
\text { Education } \\
\text { Corporation } \\
\end{array}$ & & OMPB & \\
\hline 413567 & $\begin{array}{c}\text { Graphic } \\
\text { Consultants } \\
\text { LTD. } \\
\end{array}$ & $1972-0105$ & $\begin{array}{l}\text { Popular } \\
\text { Science }\end{array}$ & $\begin{array}{c}\text { The Kingdom of } \\
\text { the Storm }\end{array}$ & 1920/1924? & us & $\begin{array}{c}\text { Scientific } \\
\text { Education } \\
\text { Corporation } \\
\end{array}$ & & OMPB & $\begin{array}{c}\text { Tinted } \\
\text { orange and } \\
\text { yellow } \\
\end{array}$ \\
\hline 413568 & $\begin{array}{c}\text { Graphic } \\
\text { Consultants } \\
\text { LTD. } \\
\end{array}$ & $1972-0105$ & & $\begin{array}{c}\text { The Bunch of } \\
\text { Violets = Le } \\
\text { Bouquet de } \\
\text { violettes } \\
\end{array}$ & 1908 & $\mathrm{fr}$ & Pathe Freres & & OMPB & \\
\hline 413569 & $\begin{array}{l}\text { Graphic } \\
\text { Consultants } \\
\text { LTD. } \\
\end{array}$ & $1972-0105$ & & $\begin{array}{c}\text { The Thief of } \\
\text { Honour }\end{array}$ & 1913 & $\mathrm{fr}$ & Pathe Freres & & OMPB & \\
\hline 413570 & $\begin{array}{l}\text { Graphic } \\
\text { Consultants } \\
\text { LTD. } \\
\end{array}$ & $1972-0105$ & & Haunted Range & 1926 & us & $\begin{array}{c}\text { Clifford S. Elfelt } \\
\text { Productions }\end{array}$ & & OMPB & \\
\hline 413571 & $\begin{array}{c}\text { Graphic } \\
\text { Consultants } \\
\text { LTD. } \\
\end{array}$ & $1972-0105$ & & $\begin{array}{c}\text { Madame Tallien = } \\
\text { Madame Tallien : } \\
1793 \text { An Episode } \\
\text { of the French } \\
\text { Revolution }\end{array}$ & 1911 & $\mathrm{fr}$ & Pathe Freres & & OMPB & \\
\hline 413604 & $\begin{array}{l}\text { Graphic } \\
\text { Consultants } \\
\text { LTD. } \\
\end{array}$ & $1972-0105$ & & A Maker of Men & 1921 & us & Plymouth & & OMPB & \\
\hline 413609 & $\begin{array}{c}\text { Graphic } \\
\text { Consultants } \\
\text { LTD. } \\
\end{array}$ & $1972-0105$ & & Shooting Stars & 1928 & uk & $\begin{array}{c}\text { British } \\
\text { International } \\
\text { Films Ltd. } \\
\end{array}$ & & & $\begin{array}{c}\text { little } \\
\text { information }\end{array}$ \\
\hline 413610 & $\begin{array}{c}\text { Graphic } \\
\text { Consultants } \\
\text { LTD. } \\
\end{array}$ & $1972-0105$ & & $\begin{array}{c}\text { Livingstone }= \\
\text { David Livingstone } \\
: \text { [incomplete] }\end{array}$ & 1925 & uk & Hero & & & $\begin{array}{c}\text { little } \\
\text { information; } \\
\text { Marmaduke } \\
\text { Arundel } \\
\text { Wetherell } \\
\text { listed as } \\
\text { creator } \\
\end{array}$ \\
\hline 413611 & $\begin{array}{l} \\
\text { Graphic } \\
\text { Consultants } \\
\text { LTD. } \\
\end{array}$ & $1972-0105$ & $\begin{array}{c}\text { Tense } \\
\text { Moments } \\
\text { from Great } \\
\text { Plays } \\
\end{array}$ & Macbeth & 1923 & uk & Artclass & & & \\
\hline 413612 & $\begin{array}{l}\text { Graphic } \\
\text { Consultants } \\
\text { LTD. } \\
\end{array}$ & $1972-0105$ & $\begin{array}{c}\text { Tense } \\
\text { Moments } \\
\text { from Opera }\end{array}$ & Maritana & 1922 & uk & $\begin{array}{c}\text { Master } \\
\text { (Gaumont) }\end{array}$ & & & \\
\hline 413613 & $\begin{array}{c}\text { Graphic } \\
\text { Consultants } \\
\text { LTD. } \\
\end{array}$ & $1972-0105$ & & $\begin{array}{l}\text { The Postman's } \\
\text { Christmas Box }\end{array}$ & $1912 / 1920 ?$ & $\mathrm{fr}$ & Pathe Freres & & OMPB & $\begin{array}{c}\text { Country of } \\
\text { production } \\
\text { is not offical } \\
\text { known. } \\
\end{array}$ \\
\hline 413614 & $\begin{array}{l}\text { Graphic } \\
\text { Consultants } \\
\text { LTD. } \\
\end{array}$ & $1972-0105$ & & $\begin{array}{c}\text { Ontario } \\
\text { Southdown Sheep } \\
\end{array}$ & 1920/1932? & $\mathrm{cn}$ & OMPB & & & \\
\hline 413616 & $\begin{array}{c}\text { Graphic } \\
\text { Consultants } \\
\text { LTD. } \\
\end{array}$ & $1972-0105$ & & $\begin{array}{c}\text { Ontario's Poultry } \\
\text { at Home }\end{array}$ & 1925/1930? & $\mathrm{cn}$ & OMPB & & OMPB & \\
\hline 413617 & $\begin{array}{l}\text { Graphic } \\
\text { Consultants } \\
\text { LTD. } \\
\end{array}$ & $1972-0105$ & & $\begin{array}{c}\text { Parasites Injurious } \\
\text { to Sheep and } \\
\text { Swine } \\
\end{array}$ & $1929 / 1930$ & $\mathrm{cn}$ & OMPB & & & \\
\hline 413618 & $\begin{array}{l}\text { Graphic } \\
\text { Consultants } \\
\text { LTD. }\end{array}$ & $1972-0105$ & & Raspberry Culture & 1919/1922? & $\mathrm{cn}$ & $\begin{array}{c}\text { Pathescope of } \\
\text { Canada }\end{array}$ & OMPB & & \\
\hline 413619 & $\begin{array}{c}\text { Graphic } \\
\text { Consultants } \\
\text { LTD. } \\
\end{array}$ & $1972-0105$ & & $\begin{array}{l}\text { The Preparation of } \\
\text { Fowl for Roasting }\end{array}$ & 1919/1921? & $\mathrm{cn}$ & $\begin{array}{l}\text { Pathescope of } \\
\text { Canada }\end{array}$ & ОМРВ & & \\
\hline 413620 & $\begin{array}{l}\text { Graphic } \\
\text { Consultants } \\
\text { LTD. } \\
\end{array}$ & $1972-0105$ & & $\begin{array}{c}\text { Old Santa Comes } \\
\text { Down From The } \\
\text { North } \\
\end{array}$ & 1930 & $\mathrm{cn}$ & OMPB & & & \\
\hline 413621 & $\begin{array}{c}\text { Graphic } \\
\text { Consultants } \\
\text { LTD. } \\
\end{array}$ & $1972-0105$ & & $\begin{array}{c}\text { Service of the Fish } \\
\text { Hatcheries }\end{array}$ & 1926 & cn & OMPB & & & \\
\hline 413622 & $\begin{array}{l}\text { Graphic } \\
\text { Consultants } \\
\text { LTD. }\end{array}$ & $1972-0105$ & & $\begin{array}{c}\text { Shorthorns at } \\
\text { Canadian National } \\
\text { Exhibition = } \\
\end{array}$ & 1919/1922? & $\mathrm{cn}$ & $\begin{array}{c}\text { Pathescope of } \\
\text { Canada }\end{array}$ & OMPB & & $\begin{array}{l}\text { Tinted } \\
\text { Yellow } \\
\end{array}$ \\
\hline
\end{tabular}




\begin{tabular}{|c|c|c|c|c|c|c|c|c|c|c|}
\hline & & & & Shorthorns at CNE & & & & & & \\
\hline 413623 & $\begin{array}{c}\text { Graphic } \\
\text { Consultants } \\
\text { LTD. }\end{array}$ & 1972-0105 & & $\begin{array}{l}\text { Shorthorns on } \\
\text { Ontario Farms }\end{array}$ & 1919/1921? & $\mathrm{cn}$ & $\begin{array}{c}\text { Pathescope of } \\
\text { Canada }\end{array}$ & OMPB & & $\begin{array}{c}\text { Tinted } \\
\text { Yellow; no } \\
\text { subject } \\
\text { description }\end{array}$ \\
\hline 413624 & $\begin{array}{c}\text { Graphic } \\
\text { Consultants } \\
\text { LTD. }\end{array}$ & $1972-0105$ & & Speckled Trout & 1932/1933? & $\mathrm{cn}$ & OMPB & & & \\
\hline 413625 & $\begin{array}{c}\text { Graphic } \\
\text { Consultants } \\
\text { LTD. }\end{array}$ & $1972-0105$ & & Spraying Mustard & 1919/1921? & $\mathrm{cn}$ & $\begin{array}{c}\text { Pathescope of } \\
\text { Canada }\end{array}$ & OMPB & & $\begin{array}{c}\text { Part tinted } \\
\text { yellow }\end{array}$ \\
\hline 413626 & $\begin{array}{c}\text { Graphic } \\
\text { Consultants } \\
\text { LTD. }\end{array}$ & $1972-0105$ & & $\begin{array}{l}\text { Strawberry } \\
\text { Culture }\end{array}$ & 1919/1921? & $\mathrm{cn}$ & $\begin{array}{c}\text { Pathescope of } \\
\text { Canada } \\
\end{array}$ & OMPB & & $\begin{array}{l}\text { Tinted } \\
\text { yellow }\end{array}$ \\
\hline 413627 & $\begin{array}{c}\text { Graphic } \\
\text { Consultants } \\
\text { LTD. }\end{array}$ & 1972-0105 & & Timber Harvest & 1925/1929? & $\mathrm{cn}$ & OMPB & & & \\
\hline 413628 & $\begin{array}{c}\text { Graphic } \\
\text { Consultants } \\
\text { LTD. }\end{array}$ & $1972-0105$ & & $\begin{array}{l}\text { Top Grafting of } \\
\text { Fruit Trees }\end{array}$ & 1919/1921? & $\mathrm{cn}$ & $\begin{array}{c}\text { Pathescope of } \\
\text { Canada }\end{array}$ & OMPB & & \\
\hline 413828 & $\begin{array}{c}\text { Graphic } \\
\text { Consultants } \\
\text { LTD. } \\
\end{array}$ & $1972-0105$ & & Snow and Ice & 1920/1924? & N/A & Pathe Freres & & & \\
\hline 413932 & $\begin{array}{c}\text { Graphic } \\
\text { Consultants } \\
\text { LTD. }\end{array}$ & $1972-0105$ & & $\begin{array}{c}\text { Nature's Tent } \\
\text { Builders }\end{array}$ & 1916 & us & $\begin{array}{c}\text { Bray } \\
\text { Productions Inc. }\end{array}$ & & & \\
\hline 413945 & $\begin{array}{c}\text { Graphic } \\
\text { Consultants } \\
\text { LTD. }\end{array}$ & $1972-0105$ & $\begin{array}{l}\text { Gem of the } \\
\text { Screen }\end{array}$ & $\begin{array}{l}\text { A Day with the } \\
\text { Gypsies }\end{array}$ & 1920/1927? & uk & Hepworth & & & $\begin{array}{c}\text { archivist } \\
\text { notes, A } \\
\text { Cranfield } \\
\text { and Clarke } \\
\text { Picture - A } \\
\text { Red Seal } \\
\text { Picture. } \\
\end{array}$ \\
\hline 413947 & $\begin{array}{c}\text { Graphic } \\
\text { Consultants } \\
\text { LTD. }\end{array}$ & $1972-0105$ & $\begin{array}{l}\text { Gem of the } \\
\text { Screen }\end{array}$ & $\begin{array}{c}\text { Shakespeare in } \\
\text { Memoriam }\end{array}$ & 1924 & N/A & $\begin{array}{l}\text { Cranfield and } \\
\text { Clarke }\end{array}$ & & $\begin{array}{c}\text { Red Seal } \\
\text { Pictures } \\
\text { Corporation }\end{array}$ & Toned \\
\hline 413948 & $\begin{array}{c}\text { Graphic } \\
\text { Consultants } \\
\text { LTD. }\end{array}$ & $1972-0105$ & & $\begin{array}{l}\text { A Social Scourge : } \\
\text { Tuberculosis } \\
\end{array}$ & 1920/1924? & $\mathrm{N} / \mathrm{A}$ & Pathe Freres & & & \\
\hline 413949 & $\begin{array}{c}\text { Graphic } \\
\text { Consultants } \\
\text { LTD. }\end{array}$ & $1972-0105$ & & Soil Physics & 1920/1921? & $\mathrm{cn}$ & $\begin{array}{c}\text { Filmcraft } \\
\text { Industries Ltd. }\end{array}$ & OMPB & & \\
\hline 413950 & $\begin{array}{c}\text { Graphic } \\
\text { Consultants } \\
\text { LTD. }\end{array}$ & $1972-0105$ & & $\begin{array}{l}\text { Diseases of } \\
\text { Vegetables }\end{array}$ & 1920/1922? & $\mathrm{cn}$ & $\begin{array}{c}\text { Pathescope of } \\
\text { Canada } \\
\end{array}$ & OMPB & & $\begin{array}{l}\text { Tinted } \\
\text { yellow }\end{array}$ \\
\hline 413951 & $\begin{array}{c}\text { Graphic } \\
\text { Consultants } \\
\text { LTD. } \\
\end{array}$ & 1972-0105 & & $\begin{array}{c}\text { Clay Tile } \\
\text { Manufacture }\end{array}$ & 1925/1926? & $\mathrm{cn}$ & OMPB & & & $\begin{array}{l}\text { Tinted } \\
\text { yellow }\end{array}$ \\
\hline 413953 & $\begin{array}{c}\text { Graphic } \\
\text { Consultants } \\
\text { LTD. }\end{array}$ & $1972-0105$ & & $\begin{array}{c}\text { Conduction of } \\
\text { Heat }\end{array}$ & 1920/1930? & us & $\begin{array}{c}\text { Bray } \\
\text { Productions Inc. }\end{array}$ & & & $\begin{array}{l}\text { Tinted } \\
\text { yellow }\end{array}$ \\
\hline 413955 & $\begin{array}{c}\text { Graphic } \\
\text { Consultants } \\
\text { LTD. } \\
\end{array}$ & 1972-0105 & & The Depth Bomb & 1918 & us & $\begin{array}{c}\text { Bray } \\
\text { Productions Inc. }\end{array}$ & & & $\begin{array}{c}\text { Tinted } \\
\text { unknown } \\
\text { colour }\end{array}$ \\
\hline 413957 & $\begin{array}{c}\text { Graphic } \\
\text { Consultants } \\
\text { LTD. } \\
\end{array}$ & 1972-0105 & & $\begin{array}{c}\text { History of } \\
\text { Weaving }\end{array}$ & 1920/1930? & $\mathrm{N} / \mathrm{A}$ & N/A & & & \\
\hline 413959 & $\begin{array}{c}\text { Graphic } \\
\text { Consultants } \\
\text { LTD. }\end{array}$ & 1972-0105 & & $\begin{array}{l}\text { How Mountains } \\
\text { Are Formed }\end{array}$ & 1920/1924? & N/A & Pathe Freres & & & \\
\hline 413960 & $\begin{array}{c}\text { Graphic } \\
\text { Consultants } \\
\text { LTD. }\end{array}$ & $1972-0105$ & & $\begin{array}{c}\text { In the Country of } \\
\text { Laos = In the } \\
\text { Country of the } \\
\text { Laos }\end{array}$ & $1920 / 1924 ?$ & N/A & Pathe Freres & & & \\
\hline
\end{tabular}




\begin{tabular}{|c|c|c|c|c|c|c|c|c|c|c|}
\hline 413961 & $\begin{array}{c}\text { Graphic } \\
\text { Consultants } \\
\text { LTD. }\end{array}$ & 1972-0105 & & $\begin{array}{l}\text { The Carrot } \\
\text { Caterpillar }\end{array}$ & 1920/1924? & N/A & Pathe Freres & & & \\
\hline 413962 & $\begin{array}{c}\text { Graphic } \\
\text { Consultants } \\
\text { LTD. } \\
\end{array}$ & 1972-0105 & & $\begin{array}{l}\text { The Selection and } \\
\text { Testing of Seed }\end{array}$ & 1919/1922? & $\mathrm{cn}$ & $\begin{array}{c}\text { Pathescope of } \\
\text { Canada } \\
\end{array}$ & OMPB & & \\
\hline 413964 & $\begin{array}{c}\text { Graphic } \\
\text { Consultants } \\
\text { LTD. }\end{array}$ & 1972-0105 & & The Broken Gate & 1927 & us & $\begin{array}{c}\text { Tiffany } \\
\text { Productions Inc. }\end{array}$ & & & \\
\hline 413965 & $\begin{array}{c}\text { Graphic } \\
\text { Consultants } \\
\text { LTD. }\end{array}$ & 1972-0105 & $\begin{array}{c}\text { Goldwyn- } \\
\text { Bray } \\
\text { Pictograph } \\
\end{array}$ & $\begin{array}{l}\text { An Adventure in } \\
\text { Tripoli } \\
\end{array}$ & 1920 & us & $\begin{array}{c}\text { Bray } \\
\text { Productions Inc. }\end{array}$ & & & $\begin{array}{l}\text { Tinted } \\
\text { unknown } \\
\text { colour } \\
\end{array}$ \\
\hline 413966 & $\begin{array}{c}\text { Graphic } \\
\text { Consultants } \\
\text { LTD. } \\
\end{array}$ & 1972-0105 & $\begin{array}{c}\text { The } \\
\text { Magazine on } \\
\text { the Screen }\end{array}$ & Paradise for Birds & 1920 & us & $\begin{array}{c}\text { Bray } \\
\text { Productions Inc. }\end{array}$ & & & \\
\hline 413967 & $\begin{array}{c}\text { Graphic } \\
\text { Consultants } \\
\text { LTD. }\end{array}$ & 1972-0105 & & Single Shot Parker & 1917 & us & $\begin{array}{c}\text { Selig-Polyscope } \\
\text { Company }\end{array}$ & & & \\
\hline 414153 & $\begin{array}{c}\text { Graphic } \\
\text { Consultants } \\
\text { LTD. } \\
\end{array}$ & 1972-0105 & & The Panama Canal & 1917 & us & $\begin{array}{c}\text { Bray } \\
\text { Productions Inc. }\end{array}$ & & & \\
\hline 414154 & $\begin{array}{c}\text { Graphic } \\
\text { Consultants } \\
\text { LTD. }\end{array}$ & 1972-0105 & & $\begin{array}{c}\text { Napoli }=[\text { Benito } \\
\text { Mussolini }]\end{array}$ & $1916 ?$ & N/A & N/A & & & \\
\hline 414289 & $\begin{array}{c}\text { Graphic } \\
\text { Consultants } \\
\text { LTD. }\end{array}$ & $1972-0105$ & & $\begin{array}{l}\text { Analysis of the } \\
\text { Movements of } \\
\text { Animals on the } \\
\text { Cinematograph }\end{array}$ & 1920/1924? & N/A & N/A & & & \\
\hline 414359 & $\begin{array}{c}\text { Graphic } \\
\text { Consultants } \\
\text { LTD. }\end{array}$ & 1972-0105 & & [Our First Flyers] & $1920 / 1925 ?$ & us & $\begin{array}{c}\text { Bray } \\
\text { Productions Inc. }\end{array}$ & & & $\begin{array}{l}\text { Tinted } \\
\text { unknown } \\
\text { colour }\end{array}$ \\
\hline 414427 & $\begin{array}{c}\text { Graphic } \\
\text { Consultants } \\
\text { LTD. }\end{array}$ & 1972-0105 & & By Orange Aid & 1918 & us & $\begin{array}{c}\text { Christie Film } \\
\text { Company }\end{array}$ & & OMPB & $\begin{array}{l}\text { Tinted } \\
\text { yellow }\end{array}$ \\
\hline 414428 & $\begin{array}{c}\text { Graphic } \\
\text { Consultants } \\
\text { LTD. }\end{array}$ & 1972-0105 & & $\begin{array}{c}\text { Candied Dainties } \\
\text { and Fresh from the } \\
\text { Ovens : } \\
\text { [incomplete] }\end{array}$ & 1922/1923? & $\mathrm{cn}$ & $\begin{array}{c}\text { Pathescope of } \\
\text { Canada }\end{array}$ & & & \\
\hline 414429 & $\begin{array}{c}\text { Graphic } \\
\text { Consultants } \\
\text { LTD. }\end{array}$ & 1972-0105 & & Carbonic Gas & 1920/1924? & $\mathrm{cn}$ & $\begin{array}{c}\text { Pathescope of } \\
\text { Canada }\end{array}$ & & OMPB & $\begin{array}{c}\text { Full title is: } \\
\text { A gas which } \\
\text { is dangerous } \\
\text { to breathe } \\
\text { but } \\
\text { inoffensive } \\
\text { to drink } \\
\text { carbonic gas }\end{array}$ \\
\hline 414430 & $\begin{array}{c}\text { Graphic } \\
\text { Consultants } \\
\text { LTD. }\end{array}$ & 1972-0105 & & Monaco & 1920/1924? & $\mathrm{fr}$ & Pathe Freres & & & \\
\hline 414700 & $\begin{array}{c}\text { Graphic } \\
\text { Consultants } \\
\text { LTD. }\end{array}$ & 1972-0105 & & $\begin{array}{l}\text { Ice Breaking in } \\
\text { Finland }\end{array}$ & 1920/1924? & N/A & Pathe Freres & & & \\
\hline 414722 & $\begin{array}{c}\text { Graphic } \\
\text { Consultants } \\
\text { LTD. }\end{array}$ & 1972-0105 & $\begin{array}{c}\text { Science and } \\
\text { Nature }\end{array}$ & $\begin{array}{l}\text { Insect Mimicry = } \\
\text { Insect Mimics }\end{array}$ & 1920/1924? & N/A & Pathe Freres & & OMPB & \\
\hline 414735 & $\begin{array}{c}\text { Graphic } \\
\text { Consultants } \\
\text { LTD. } \\
\end{array}$ & 1972-0105 & & Sea Flowers & 1920/1924? & N/A & Pathe Freres & & & \\
\hline 414810 & $\begin{array}{c}\text { Graphic } \\
\text { Consultants } \\
\text { LTD. }\end{array}$ & 1972-0105 & & A Trip to Mortain & 1920/1924? & $\mathrm{fr}$ & Pathe Freres & & & \\
\hline 414811 & $\begin{array}{c}\text { Graphic } \\
\text { Consultants } \\
\text { LTD. } \\
\end{array}$ & 1972-0105 & & $\begin{array}{c}\text { The Valley of } \\
\text { Arlberg }\end{array}$ & 1920/1924? & $\mathrm{fr}$ & Pathe Freres & & & \\
\hline 414812 & $\begin{array}{c}\text { Graphic } \\
\text { Consultants } \\
\text { LTD. }\end{array}$ & $1972-0105$ & & $\begin{array}{l}\text { The } \\
\text { Swasamundram } \\
\text { Falls }\end{array}$ & 1920/1924? & N/A & Pathe Freres & & & \\
\hline
\end{tabular}




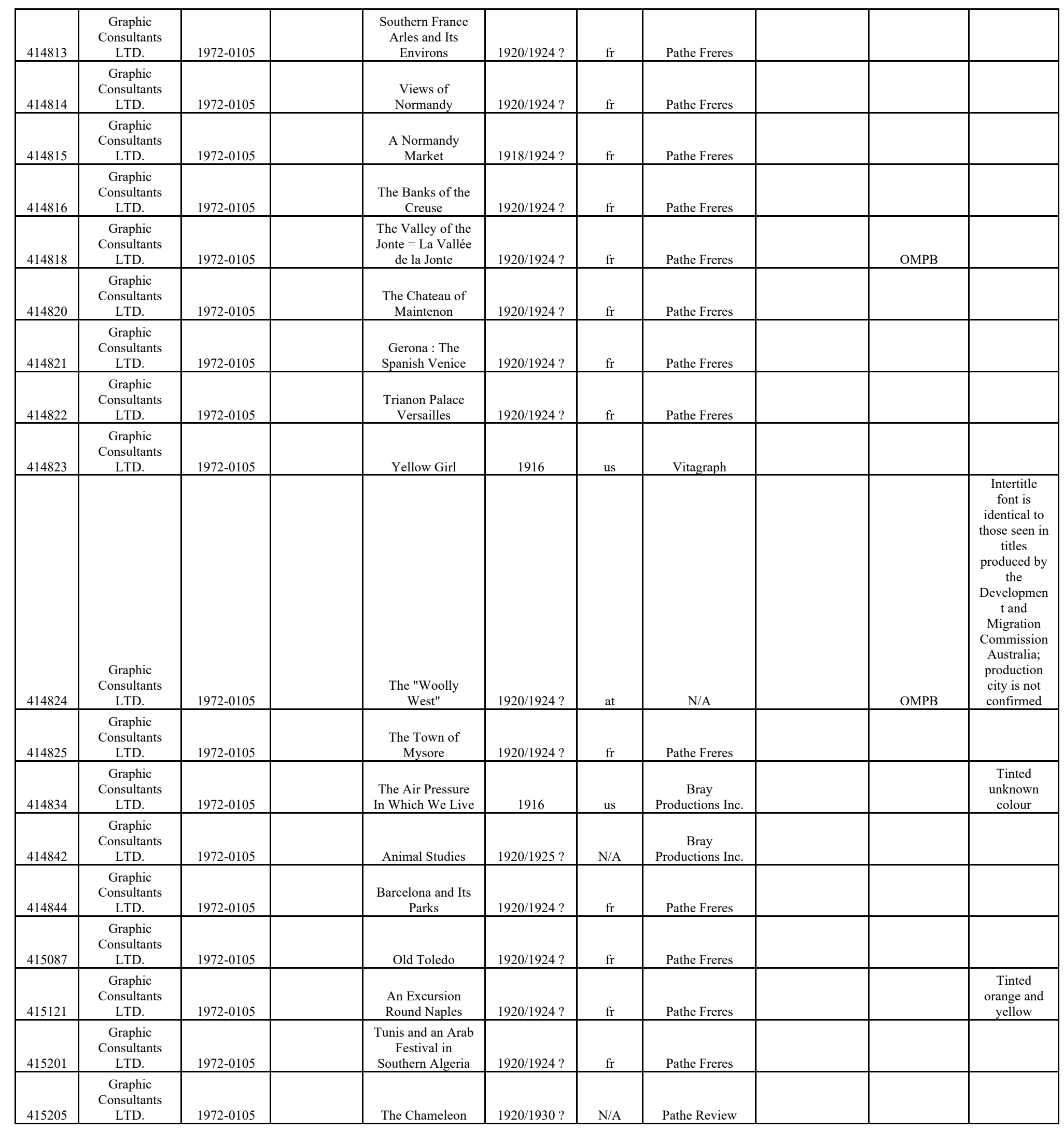




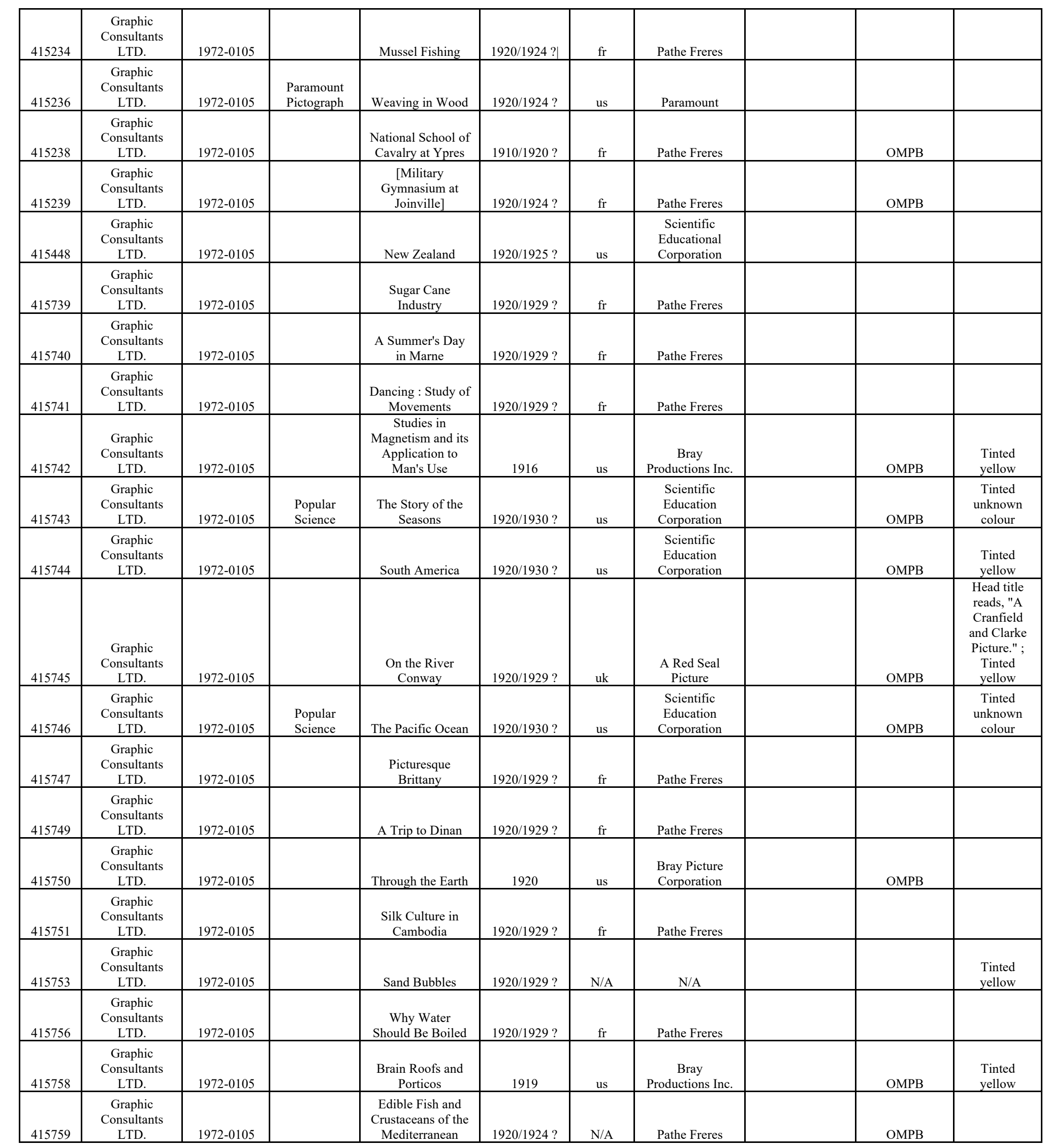




\begin{tabular}{|c|c|c|c|c|c|c|c|c|c|c|}
\hline 415760 & $\begin{array}{c}\text { Graphic } \\
\text { Consultants } \\
\text { LTD. }\end{array}$ & 1972-0105 & & $\begin{array}{c}\text { Developing } \\
\text { Natural Resource : } \\
\text { [incomplete] }\end{array}$ & 1922 & $\mathrm{cn}$ & $\begin{array}{c}\text { Filmcraft } \\
\text { Industries Ltd. }\end{array}$ & OMPB & & \\
\hline 415761 & $\begin{array}{c}\text { Graphic } \\
\text { Consultants } \\
\text { LTD. } \\
\end{array}$ & 1972-0105 & & $\begin{array}{c}\text { The Earth-Nut } \\
\text { Season at } \\
\text { Pondichery }\end{array}$ & 1920/1924? & $\mathrm{fr}$ & Pathe Freres & & & \\
\hline 415763 & $\begin{array}{c}\text { Graphic } \\
\text { Consultants } \\
\text { LTD. }\end{array}$ & $1972-0105$ & & $\begin{array}{c}\text { [Cultivation of } \\
\text { Rubber in Malay] }\end{array}$ & 1920/1924? & $\mathrm{fr}$ & Pathe Freres & & & \\
\hline 415764 & $\begin{array}{c}\text { Graphic } \\
\text { Consultants } \\
\text { LTD. }\end{array}$ & 1972-0105 & & $\begin{array}{c}\text { Every Swimmer a } \\
\text { Life Saver }\end{array}$ & 1920/1930? & us & $\begin{array}{c}\text { American Red } \\
\text { Cross }\end{array}$ & & & \\
\hline 415769 & $\begin{array}{c}\text { Graphic } \\
\text { Consultants } \\
\text { LTD. } \\
\end{array}$ & 1972-0105 & $\begin{array}{c}\text { The } \\
\text { Magazine on } \\
\text { the Screen }\end{array}$ & $\begin{array}{l}\text { Feeding the } \\
\text { Microscope }\end{array}$ & 1921 & us & $\begin{array}{c}\text { Bray } \\
\text { Productions Inc. }\end{array}$ & & & $\begin{array}{l}\text { Tinted } \\
\text { yellow } \\
\end{array}$ \\
\hline 415773 & $\begin{array}{c}\text { Graphic } \\
\text { Consultants } \\
\text { LTD. }\end{array}$ & $1972-0105$ & & $\begin{array}{c}\text { Crayfish of } \\
\text { Tasmania }\end{array}$ & 1920/1924? & $\mathrm{N} / \mathrm{A}$ & $\mathrm{N} / \mathrm{A}$ & & OMPB & \\
\hline 415775 & $\begin{array}{c}\text { Graphic } \\
\text { Consultants } \\
\text { LTD. } \\
\end{array}$ & 1972-0105 & & The Hermit Crab & 1920/1924? & $\mathrm{N} / \mathrm{A}$ & Pathe Freres & & & \\
\hline 415776 & $\begin{array}{c}\text { Graphic } \\
\text { Consultants } \\
\text { LTD. }\end{array}$ & 1972-0105 & & $\begin{array}{c}\text { In a Sculptor's } \\
\text { Studio } \\
\end{array}$ & 1920/1930? & us & $\mathrm{N} / \mathrm{A}$ & & & $\begin{array}{c}\text { Tinted } \\
\text { unknown } \\
\text { colour } \\
\end{array}$ \\
\hline 415858 & $\begin{array}{c}\text { Graphic } \\
\text { Consultants } \\
\text { LTD. }\end{array}$ & $1972-0105$ & & $\begin{array}{c}\text { Africa : } \\
\text { [incomplete] }\end{array}$ & $1920 / 1930 ?$ & us & $\begin{array}{c}\text { Scientific } \\
\text { Educational } \\
\text { Corporation } \\
\end{array}$ & & & \\
\hline 415859 & $\begin{array}{c}\text { Graphic } \\
\text { Consultants } \\
\text { LTD. }\end{array}$ & $1972-0105$ & & $\begin{array}{c}\text { Europe and Asia : } \\
\text { [incomplete] }\end{array}$ & 1920/1930? & us & $\begin{array}{c}\text { Scientific } \\
\text { Educational } \\
\text { Corporation } \\
\end{array}$ & & OMPB & \\
\hline 415860 & $\begin{array}{c}\text { Graphic } \\
\text { Consultants } \\
\text { LTD. }\end{array}$ & 1972-0105 & & $\begin{array}{c}\text { Bits of Japanese } \\
\text { Life } \\
\end{array}$ & 1920/1924? & $\mathrm{N} / \mathrm{A}$ & $\begin{array}{c}\text { Cranfield and } \\
\text { Clarke }\end{array}$ & & & \\
\hline 415861 & $\begin{array}{c}\text { Graphic } \\
\text { Consultants } \\
\text { LTD. }\end{array}$ & 1972-0105 & & $\begin{array}{l}\text { Chinese Porcelain } \\
\text { and Art Pottery at } \\
\text { Kai-Kai (Saigon) }\end{array}$ & 1920/1924? & $\mathrm{fr}$ & Pathe Freres & & OMPB & $\begin{array}{c}\text { Pathe } \\
\text { possible } \\
\text { production } \\
\text { company } \\
\end{array}$ \\
\hline 415862 & $\begin{array}{c}\text { Graphic } \\
\text { Consultants } \\
\text { LTD. }\end{array}$ & $1972-0105$ & & $\begin{array}{c}\text { Dangerous } \\
\text { Animals }\end{array}$ & 1920/1930? & us & $\begin{array}{c}\text { Bray } \\
\text { Productions Inc. }\end{array}$ & & & $\begin{array}{c}\text { Tinted } \\
\text { unknown } \\
\text { colour } \\
\end{array}$ \\
\hline 415863 & $\begin{array}{c}\text { Graphic } \\
\text { Consultants } \\
\text { LTD. } \\
\end{array}$ & $1972-0105$ & & $\begin{array}{c}\text { The Debt: } \\
\text { Episode of the } \\
\text { Massacre of St. } \\
\text { Bartholomew } \\
1572 \\
\end{array}$ & $1917 ?$ & $\mathrm{fr}$ & Pathe Freres & & OMPB & $\begin{array}{l}\text { Country of } \\
\text { production } \\
\text { questioned } \\
\end{array}$ \\
\hline 415864 & $\begin{array}{c}\text { Graphic } \\
\text { Consultants } \\
\text { LTD. }\end{array}$ & 1972-0105 & & $\begin{array}{c}\text { Desert Island = } \\
\text { Dessert Island }\end{array}$ & 1920/1924? & N/A & N/A & & OMPB & $\begin{array}{c}\text { Listed in } \\
1931 \text { OMPB } \\
\text { catalogue as } \\
\text { DESSERT } \\
\text { ISLAND }\end{array}$ \\
\hline 415865 & $\begin{array}{c}\text { Graphic } \\
\text { Consultants } \\
\text { LTD. }\end{array}$ & 1972-0105 & & $\begin{array}{c}\text { An Escapade of } \\
\text { Marie Antoinette }\end{array}$ & 1908 & $\mathrm{fr}$ & Pathe Freres & & OMPB & \\
\hline 415866 & $\begin{array}{c}\text { Graphic } \\
\text { Consultants } \\
\text { LTD. }\end{array}$ & $1972-0105$ & & Harvesting Salt & 1920/1924? & $\mathrm{N} / \mathrm{A}$ & $\mathrm{N} / \mathrm{A}$ & & & \\
\hline 415868 & $\begin{array}{c}\text { Graphic } \\
\text { Consultants } \\
\text { LTD. }\end{array}$ & $1972-0105$ & & $\begin{array}{l}\text { The History of a } \\
\text { Grain of Wheat }\end{array}$ & 1920/1922? & $\mathrm{cn}$ & $\begin{array}{c}\text { Filmcraft } \\
\text { Industries Ltd. }\end{array}$ & OMPB & & $\begin{array}{c}\text { CGMPB } \\
\text { similar title } \\
\text { "The story } \\
\text { of a grain of } \\
\text { wheat" }\end{array}$ \\
\hline 415869 & $\begin{array}{c}\text { Graphic } \\
\text { Consultants } \\
\text { LTD. }\end{array}$ & $1972-0105$ & & $\begin{array}{l}\text { How Champagne } \\
\text { is Made at } \\
\text { Epernay }\end{array}$ & 1920/1924? & fr & Pathe Freres & & OMPB & \\
\hline 415871 & $\begin{array}{c}\text { Graphic } \\
\text { Consultants } \\
\text { LTD. }\end{array}$ & $1972-0105$ & $\begin{array}{l}\text { Goldwyn- } \\
\text { Bray- } \\
\text { Pictograph }\end{array}$ & The Human Voice & 1920 & us & $\begin{array}{c}\text { Bray } \\
\text { Productions Inc. }\end{array}$ & & OMPB & $\begin{array}{c}\text { Tinted Red. } \\
\text { Makes use } \\
\text { of X-Ray } \\
\text { footage. }\end{array}$ \\
\hline
\end{tabular}




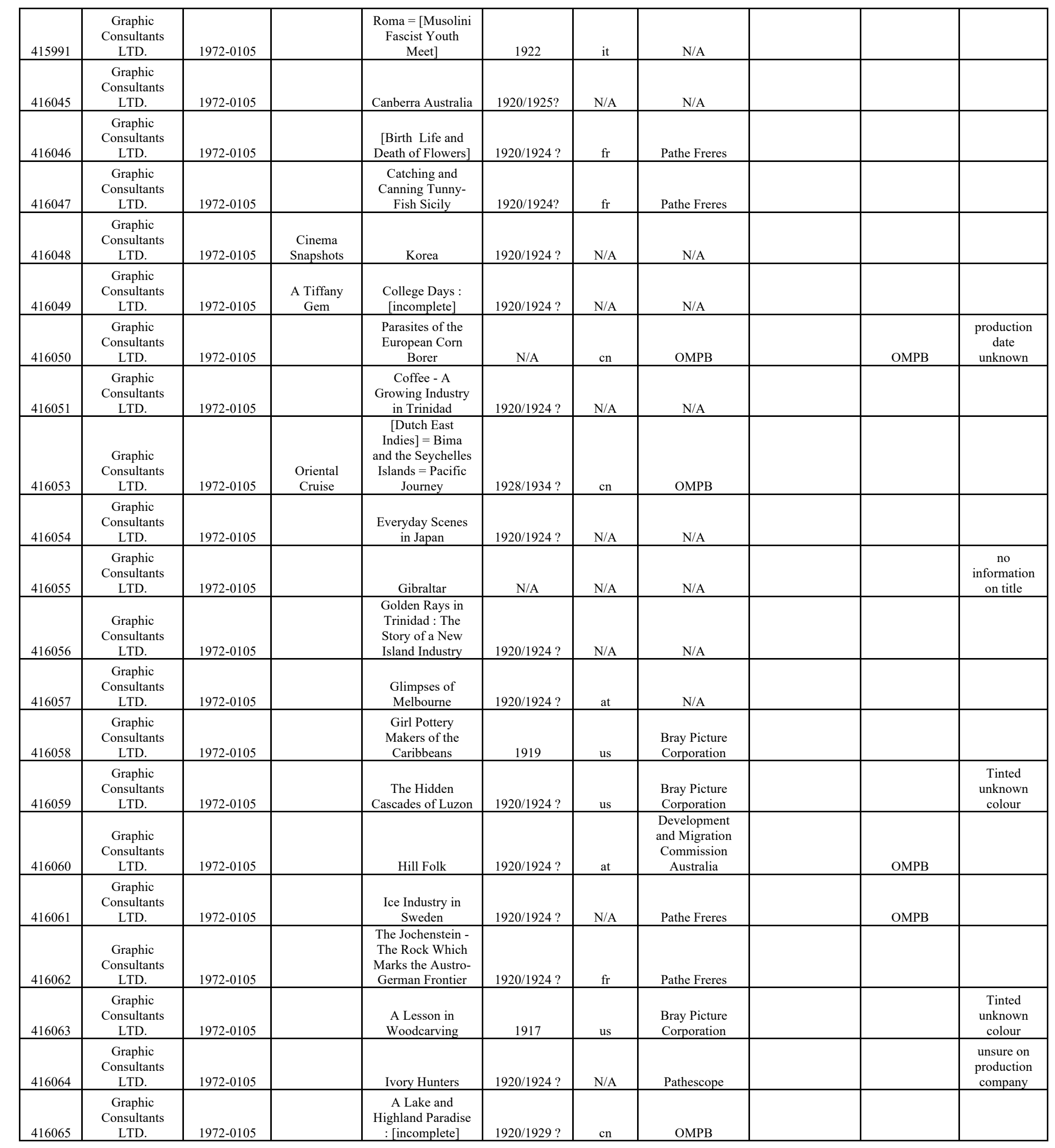




\begin{tabular}{|c|c|c|c|c|c|c|c|c|c|}
\hline 416066 & $\begin{array}{c}\text { Graphic } \\
\text { Consultants } \\
\text { LTD. } \\
\end{array}$ & 1972-0105 & & $\begin{array}{c}\text { A Lofty Soul: } \\
\text { Sentimental } \\
\text { Comedy = [Une } \\
\text { Grande d'ame }]\end{array}$ & 1920/1924? & $\mathrm{fr}$ & Pathe Freres & & \\
\hline 416067 & $\begin{array}{c}\text { Graphic } \\
\text { Consultants } \\
\text { LTD. } \\
\end{array}$ & 1972-0105 & & $\begin{array}{l}\text { The Samourai's } \\
\text { Punishment: } \\
\text { Japanese } \\
\text { Pantomime } \\
\end{array}$ & 1920/1924? & $\mathrm{fr}$ & Pathe Freres & & \\
\hline 416068 & $\begin{array}{c}\text { Graphic } \\
\text { Consultants } \\
\text { LTD. } \\
\end{array}$ & 1972-0105 & & $\begin{array}{c}\text { South African } \\
\text { Fruits = Fruit } \\
\text { Harvest in South } \\
\text { Africa } \\
\end{array}$ & 1920/1924? & N/A & N/A & & \\
\hline 416069 & $\begin{array}{c}\text { Graphic } \\
\text { Consultants } \\
\text { LTD. } \\
\end{array}$ & 1972-0105 & $\begin{array}{c}\text { Daypho- } \\
\text { Bray } \\
\text { Pictograph } \\
\end{array}$ & $\begin{array}{c}\text { Song Birds as } \\
\text { Citizens : } \\
\text { [incomplete] }\end{array}$ & 1920/1930? & us & $\begin{array}{l}\text { Bray Picture } \\
\text { Corporation }\end{array}$ & & $\begin{array}{c}\text { Tinted } \\
\text { unknown } \\
\text { colour } \\
\end{array}$ \\
\hline 416070 & $\begin{array}{c}\text { Graphic } \\
\text { Consultants } \\
\text { LTD. } \\
\end{array}$ & 1972-0105 & & $\begin{array}{c}\text { The Manufacture } \\
\text { of Cheese in } \\
\text { Holland }\end{array}$ & 1920/1924? & $\mathrm{fr}$ & Pathe Freres & OMPB & \\
\hline 416072 & $\begin{array}{c}\text { Graphic } \\
\text { Consultants } \\
\text { LTD. } \\
\end{array}$ & 1972-0105 & & $\begin{array}{c}\text { Manchester Ship } \\
\text { Canal : } \\
\text { [incomplete] } \\
\end{array}$ & 1920/1924? & $\mathrm{cn}$ & $\begin{array}{c}\text { Federation of } \\
\text { British Industries }\end{array}$ & & \\
\hline 416074 & $\begin{array}{c}\text { Graphic } \\
\text { Consultants } \\
\text { LTD. } \\
\end{array}$ & 1972-0105 & & $\begin{array}{c}\text { The Water Beetle } \\
\text { and Its Larva }\end{array}$ & 1920/1924? & fr & Pathe Freres & & \\
\hline 416076 & $\begin{array}{c}\text { Graphic } \\
\text { Consultants } \\
\text { LTD. } \\
\end{array}$ & 1972-0105 & & $\begin{array}{l}\text { Venice of the } \\
\text { Orient: } \\
\text { [incomplete] }\end{array}$ & 1920/1924? & us & $\begin{array}{c}\text { Bray } \\
\text { Productions Inc. }\end{array}$ & OMPB & \\
\hline 416077 & $\begin{array}{c}\text { Graphic } \\
\text { Consultants } \\
\text { LTD. } \\
\end{array}$ & 1972-0105 & & $\begin{array}{c}\text { The Beggar of } \\
\text { Cawnpore }\end{array}$ & 1916 & N/A & Triangle & & \\
\hline 416078 & $\begin{array}{c}\text { Graphic } \\
\text { Consultants } \\
\text { LTD. } \\
\end{array}$ & 1972-0105 & & Colombo & 1919/1921? & N/A & $\mathrm{N} / \mathrm{A}$ & & \\
\hline 416247 & $\begin{array}{c}\text { Graphic } \\
\text { Consultants } \\
\text { LTD. }\end{array}$ & 1972-0105 & & $\begin{array}{c}\text { In the Dutch East } \\
\text { Indies }\end{array}$ & $1928 ?$ & $\mathrm{cn}$ & OMPB & & \\
\hline 416254 & $\begin{array}{c}\text { Graphic } \\
\text { Consultants } \\
\text { LTD. } \\
\end{array}$ & 1972-0105 & $\begin{array}{c}\text { Pathés } \\
\text { Frères' } \\
\text { Instructive } \\
\text { Series } \\
\end{array}$ & $\begin{array}{c}\text { The Electrotype } \\
\text { Process }\end{array}$ & 1920/1924? & $\mathrm{fr}$ & Pathe Freres & OMPB & \\
\hline 416256 & $\begin{array}{c}\text { Graphic } \\
\text { Consultants } \\
\text { LTD. } \\
\end{array}$ & 1972-0105 & & $\begin{array}{l}\text { Excursion in the } \\
\text { Forest of } \\
\text { Fontainebleau }\end{array}$ & 1920/1924? & $\mathrm{fr}$ & Pathe Freres & & \\
\hline 416257 & $\begin{array}{c}\text { Graphic } \\
\text { Consultants } \\
\text { LTD. } \\
\end{array}$ & 1972-0105 & $\begin{array}{c}\text { Gem of the } \\
\text { Screen }\end{array}$ & $\begin{array}{c}\text { Minnie and Me : } \\
\text { [incomplete] }\end{array}$ & 1920/1924? & N/A & $\begin{array}{c}\text { Cranfield and } \\
\text { Clarke }\end{array}$ & OMPB & $\begin{array}{l}\text { Mix of } \\
\text { tinted and } \\
\text { tones }\end{array}$ \\
\hline 416258 & $\begin{array}{c}\text { Graphic } \\
\text { Consultants } \\
\text { LTD. }\end{array}$ & 1972-0105 & & $\begin{array}{c}\text { [First Families of } \\
\text { America] }\end{array}$ & 1920/1928? & us & $\begin{array}{c}\text { Bray Studios } \\
\text { Inc. } \\
\end{array}$ & & $\begin{array}{l}\text { Tinted } \\
\text { unknown } \\
\text { colour } \\
\end{array}$ \\
\hline 416259 & $\begin{array}{c}\text { Graphic } \\
\text { Consultants } \\
\text { LTD. } \\
\end{array}$ & 1972-0105 & & $\begin{array}{c}\text { Florida Alligator } \\
\text { Farm }\end{array}$ & 1920/1928? & us & $\begin{array}{c}\text { Bray Studios } \\
\text { Inc. } \\
\end{array}$ & & \\
\hline 416260 & $\begin{array}{c}\text { Graphic } \\
\text { Consultants } \\
\text { LTD. } \\
\end{array}$ & 1972-0105 & & $\begin{array}{c}\text { Getting a Line on } \\
\text { Mr. Alexander } \\
\text { Alligator } \\
\end{array}$ & 1920/1924? & $\mathrm{fr}$ & Pathe Freres & & \\
\hline 416261 & $\begin{array}{c}\text { Graphic } \\
\text { Consultants } \\
\text { LTD. }\end{array}$ & $1972-0105$ & & $\begin{array}{l}\text { Workmates of } \\
\text { Sheepman }\end{array}$ & $1920 / 1930 ?$ & $\mathrm{~N} / \mathrm{A}$ & N/A & & \\
\hline 416262 & $\begin{array}{c}\text { Graphic } \\
\text { Consultants } \\
\text { LTD. } \\
\end{array}$ & 1972-0105 & & [Wooden Shoes] & 1920/1924? & $\mathrm{N} / \mathrm{A}$ & $\mathrm{N} / \mathrm{A}$ & OMPB & $\begin{array}{c}\text { Tinted/toned } \\
\text { green, blue, } \\
\text { yellow, } \\
\text { orange } \\
\end{array}$ \\
\hline 416263 & $\begin{array}{c}\text { Graphic } \\
\text { Consultants } \\
\text { LTD. }\end{array}$ & 1972-0105 & & $\begin{array}{c}\text { The Vagabond : } \\
\text { [incomplete] }\end{array}$ & 1916 & us & $\mathrm{N} / \mathrm{A}$ & & $\begin{array}{l}\text { Charlie } \\
\text { Chaplin as } \\
\text { uncredited }\end{array}$ \\
\hline 416265 & $\begin{array}{c}\text { Graphic } \\
\text { Consultants } \\
\text { LTD. }\end{array}$ & 1972-0105 & $\begin{array}{c}\text { The } \\
\text { Magazine on } \\
\text { the Screen }\end{array}$ & $\begin{array}{c}\text { Tree Top Concert } \\
\text { Singers: } \\
\text { [incomplete] }\end{array}$ & 1920 & us & $\begin{array}{c}\text { Bray } \\
\text { Productions Inc. }\end{array}$ & OMPB & \\
\hline
\end{tabular}




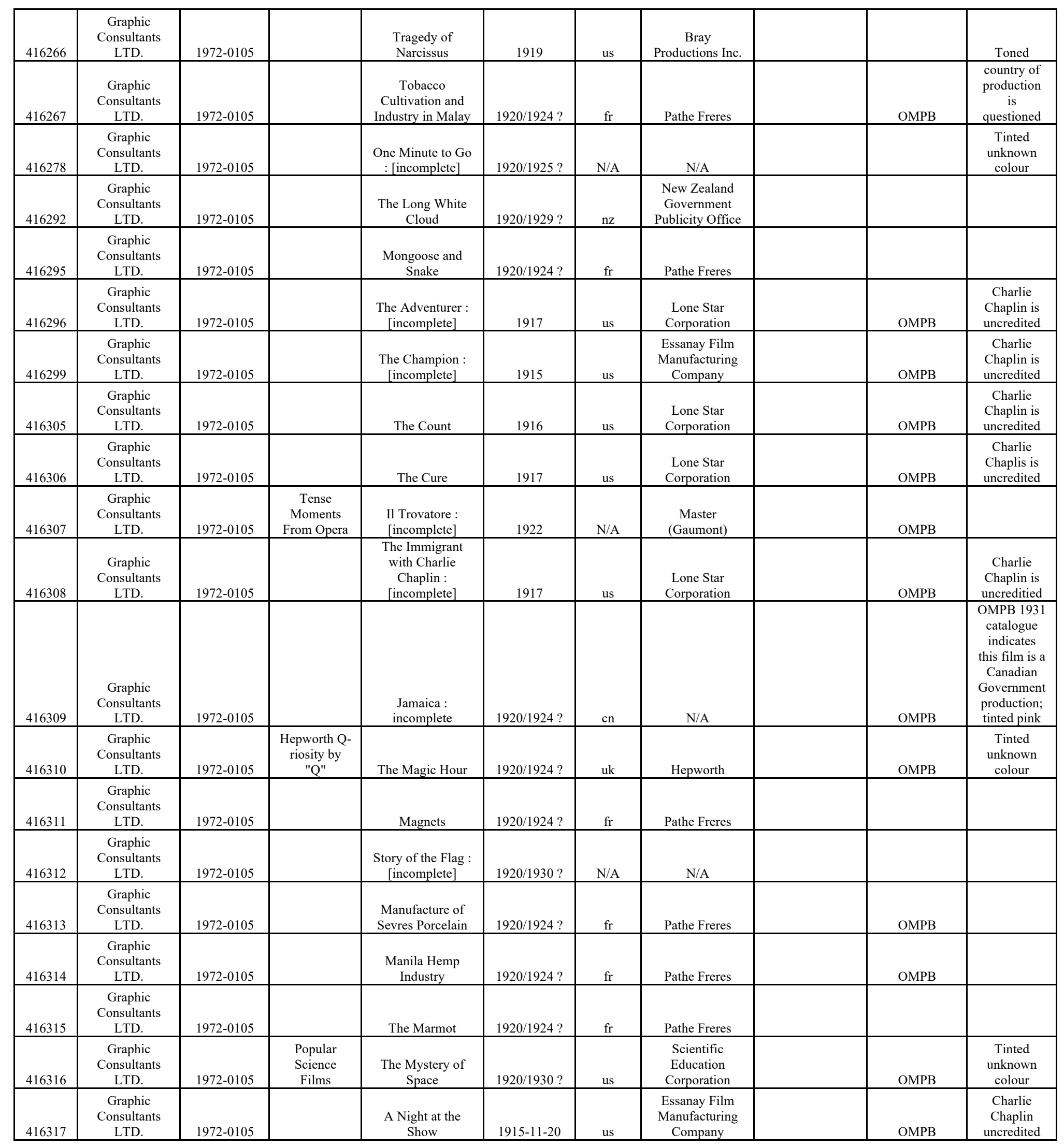




\begin{tabular}{|c|c|c|c|c|c|c|c|c|c|c|}
\hline 416318 & $\begin{array}{c}\text { Graphic } \\
\text { Consultants } \\
\text { LTD. } \\
\end{array}$ & 1972-0105 & & The Octopus & 1920/1925? & $\mathrm{fr}$ & Pathe Freres & & & \\
\hline 416324 & $\begin{array}{c}\text { Graphic } \\
\text { Consultants } \\
\text { LTD. } \\
\end{array}$ & 1972-0105 & & $\begin{array}{c}\text { Orchard } \\
\text { Management }\end{array}$ & $1927 / 1928 ?$ & $\mathrm{cn}$ & OMPB & & & \\
\hline 416325 & $\begin{array}{c}\text { Graphic } \\
\text { Consultants } \\
\text { LTD. } \\
\end{array}$ & 1972-0105 & & $\begin{array}{c}\text { Training } \\
\text { Homemakers : } \\
\text { [incomplete] }\end{array}$ & 1922 & $\mathrm{cn}$ & $\begin{array}{c}\text { Filmcraft } \\
\text { Industries Ltd. }\end{array}$ & OMPB & & $\begin{array}{l}\text { Tinted } \\
\text { unknown } \\
\text { colour } \\
\end{array}$ \\
\hline 416326 & $\begin{array}{l} \\
\text { Graphic } \\
\text { Consultants } \\
\text { LTD. } \\
\end{array}$ & 1972-0105 & $\begin{array}{c} \\
\text { Oriental } \\
\text { Cruise Series } \\
\end{array}$ & $\begin{array}{c}\text { Through Panama } \\
\text { to the Land of the } \\
\text { Incas : } \\
\text { [incomplete] }\end{array}$ & $1929 ?$ & $\mathrm{cn}$ & OMPB & & & $\begin{array}{c}\text { Photography } \\
\text { : George } \\
\text { Rutherford; } \\
\text { footage of } \\
\text { Machu } \\
\text { Picchu a } \\
\text { decade after } \\
\text { it was found } \\
\text { in } 1911 \\
\end{array}$ \\
\hline 416327 & $\begin{array}{c}\text { Graphic } \\
\text { Consultants } \\
\text { LTD. } \\
\end{array}$ & 1972-0105 & & $\begin{array}{l}\text { A Plant With } \\
\text { Nerves : The } \\
\text { Sensitive Plant } \\
\end{array}$ & 1920/1924? & $\mathrm{fr}$ & Pathe Freres & & OMPB & $\begin{array}{l}\text { production } \\
\text { country } \\
\text { questioned }\end{array}$ \\
\hline 416328 & $\begin{array}{c}\text { Graphic } \\
\text { Consultants } \\
\text { LTD. } \\
\end{array}$ & 1972-0105 & & $\begin{array}{c}\text { Les Misérables : } \\
\text { [incomplete] }\end{array}$ & 1912/1913? & $\mathrm{fr}$ & Pathe Freres & & OMPB & \\
\hline 416331 & $\begin{array}{c}\text { Graphic } \\
\text { Consultants } \\
\text { LTD. } \\
\end{array}$ & 1972-0105 & $\begin{array}{l}\text { Popular } \\
\text { Science }\end{array}$ & $\begin{array}{l}\text { Worlds in the } \\
\text { Making = } \\
\text { Animated Maps } \\
\text { and Diagrams : } \\
\text { Worlds in the } \\
\text { Making }\end{array}$ & $1920 / 1930 ?$ & us & $\begin{array}{c}\text { Scientific } \\
\text { Education } \\
\text { Corporation } \\
\end{array}$ & & OMPB & $\begin{array}{c}\text { Tinted } \\
\text { unknown } \\
\text { colour; It } \\
\text { uses actual } \\
\text { photographs } \\
\text { of the } \\
\text { different } \\
\text { forms of } \\
\text { nebula. } \\
\end{array}$ \\
\hline 416332 & $\begin{array}{c}\text { Graphic } \\
\text { Consultants } \\
\text { LTD. } \\
\end{array}$ & 1972-0105 & $\begin{array}{l}\text { Popular } \\
\text { Science }\end{array}$ & $\begin{array}{c}\text { The Earth as a } \\
\text { Whole = World as } \\
\text { a Whole : } \\
\text { [incomplete] }\end{array}$ & 1920/1930? & us & $\begin{array}{c}\text { Scientific } \\
\text { Education } \\
\text { Corporation } \\
\end{array}$ & & & Toned \\
\hline 416334 & $\begin{array}{c}\text { Graphic } \\
\text { Consultants } \\
\text { LTD. } \\
\end{array}$ & 1972-0105 & & $\begin{array}{l}\text { Wool from the } \\
\text { Fleece to the } \\
\text { Finished Product : } \\
\text { [incomplete] }\end{array}$ & 1920/1924? & $\mathrm{cn}$ & $\begin{array}{c}\text { Pathescope of } \\
\text { Canada }\end{array}$ & OMPB & & \\
\hline 416339 & $\begin{array}{c}\text { Graphic } \\
\text { Consultants } \\
\text { LTD. } \\
\end{array}$ & 1972-0105 & & $\begin{array}{c}\text { Spectacular } \\
\text { Experiments in } \\
\text { Crystallization } \\
\end{array}$ & $1920 / 1930$ & us & $\begin{array}{c}\text { Bray } \\
\text { Productions Inc. }\end{array}$ & & & $\begin{array}{c}\text { Tinted } \\
\text { yellow/blue/ } \\
\text { rose } \\
\end{array}$ \\
\hline 416340 & $\begin{array}{c}\text { Graphic } \\
\text { Consultants } \\
\text { LTD. } \\
\end{array}$ & 1972-0105 & & $\begin{array}{l}\text { Saplings in the } \\
\text { Sun }\end{array}$ & 1931 & at & N/A & & OMPB & \\
\hline 416341 & $\begin{array}{c}\text { Graphic } \\
\text { Consultants } \\
\text { LTD. } \\
\end{array}$ & 1972-0105 & & $\begin{array}{l}\text { Salt Industry in } \\
\text { Sicily }\end{array}$ & 1920/1924? & $\mathrm{fr}$ & Pathe Freres & & OMPB & $\begin{array}{l}\text { country of } \\
\text { production } \\
\text { questioned }\end{array}$ \\
\hline 416342 & $\begin{array}{c}\text { Graphic } \\
\text { Consultants } \\
\text { LTD. } \\
\end{array}$ & 1972-0105 & & $\begin{array}{c}\text { The Rudiments of } \\
\text { Flying }\end{array}$ & 1917 & us & $\begin{array}{c}\text { Paramount-Bray } \\
\text { Pictographs }\end{array}$ & & OMPB & \\
\hline 416343 & $\begin{array}{c}\text { Graphic } \\
\text { Consultants } \\
\text { LTD. } \\
\end{array}$ & 1972-0105 & & $\begin{array}{c}\text { Rambling Around } \\
\text { Old Japan }\end{array}$ & $1920 / 1925 ?$ & uk & $\begin{array}{c}\text { Cranfield and } \\
\text { Clarke }\end{array}$ & & OMPB & \\
\hline 416359 & $\begin{array}{c}\text { Graphic } \\
\text { Consultants } \\
\text { LTD. } \\
\end{array}$ & 1972-0105 & & $\begin{array}{c}\text { The Fireman } \\
1916 \\
\end{array}$ & 1916-06-12 & us & $\begin{array}{c}\text { Lone Star } \\
\text { Corporation } \\
\end{array}$ & & & $\begin{array}{c}\text { Charlie } \\
\text { Chaplin } \\
\text { uncredited }\end{array}$ \\
\hline 416400 & $\begin{array}{c}\text { Graphic } \\
\text { Consultants } \\
\text { LTD. } \\
\end{array}$ & 1972-0105 & & A Regular Fellow & 1919 & us & $\begin{array}{c}\text { Triangle-Kay } \\
\text { Bee } \\
\end{array}$ & & & \\
\hline 416401 & $\begin{array}{c}\text { Graphic } \\
\text { Consultants } \\
\text { LTD. } \\
\end{array}$ & 1972-0105 & & $\begin{array}{l}\text { Playground of the } \\
\text { Sea Folk }\end{array}$ & 1920/1925? & at & N/A & $\begin{array}{c}\text { Australia } \\
\text { Development } \\
\text { and Migration } \\
\text { Commission } \\
\end{array}$ & & \\
\hline 416407 & $\begin{array}{c}\text { Graphic } \\
\text { Consultants } \\
\text { LTD. } \\
\end{array}$ & 1972-0105 & & $\begin{array}{c}\text { Ontario Onion } \\
\text { Growers Co- } \\
\text { operative Limited } \\
=\text { Ontario Onion }\end{array}$ & $1932 ?$ & $\mathrm{cn}$ & OMPB & & & $\begin{array}{c}\text { production } \\
\text { company } \\
\text { listed "A } \\
\text { Province of }\end{array}$ \\
\hline
\end{tabular}




\begin{tabular}{|c|c|c|c|c|c|c|c|c|c|c|}
\hline & & & & $\begin{array}{c}\text { Growers Co- } \\
\text { operative Society }\end{array}$ & & & & & & $\begin{array}{l}\text { Ontario } \\
\text { Picture" }\end{array}$ \\
\hline 416408 & $\begin{array}{c}\text { Graphic } \\
\text { Consultants } \\
\text { LTD. } \\
\end{array}$ & 1972-0105 & & Quarrying Power & 1920/1924? & at & $\begin{array}{c}\text { Development } \\
\text { and Migration } \\
\text { Commission } \\
\text { Australia }\end{array}$ & & & \\
\hline 417411 & $\begin{array}{l}\text { Graphic } \\
\text { Consultants } \\
\text { LTD. } \\
\end{array}$ & 1972-0105 & & Army Review & 1914/1919? & $\mathrm{fr}$ & Pathe Freres & & & \\
\hline 418475 & $\begin{array}{l}\text { Graphic } \\
\text { Consultants } \\
\text { LTD. } \\
\end{array}$ & 1972-0105 & & $\begin{array}{l}\text { The Barn Raising } \\
\text { at Woodbridge }\end{array}$ & 1930 & $\mathrm{cn}$ & OMPB & & & \\
\hline 424762 & $\begin{array}{l}\text { Graphic } \\
\text { Consultants } \\
\text { LTD. } \\
\end{array}$ & $1972-0105$ & $\begin{array}{c}\text { Canadian } \\
\text { Sea Fowl } \\
\text { No. } 5 \\
\end{array}$ & $\begin{array}{c}\text { Birds Rare and } \\
\text { Fantastic }= \\
\text { Oiseaux rares et } \\
\text { bizarres } \\
\end{array}$ & $1934 ?$ & $\mathrm{cn}$ & CGMPB & $\begin{array}{l}\text { National Parks } \\
\text { of Canada, } \\
\text { Department of } \\
\text { Mines and } \\
\text { Resources } \\
\end{array}$ & & $\begin{array}{l}\text { Toned } \\
\text { unknow } \\
\text { colour }\end{array}$ \\
\hline 425136 & $\begin{array}{c}\text { Graphic } \\
\text { Consultants } \\
\text { LTD. }\end{array}$ & 1972-0105 & $\begin{array}{c}\text { Canadian } \\
\text { Sea Fowl } \\
\text { No. } 1\end{array}$ & $\begin{array}{c}\text { Home of the Birds } \\
=\text { Le Domaine des } \\
\text { oiseaux : } \\
\text { [incomplete] }\end{array}$ & 1930 & $\mathrm{cn}$ & CGMPB & $\begin{array}{l}\text { National Parks } \\
\text { of Canada, } \\
\text { Department of } \\
\text { the Interior }\end{array}$ & & $\begin{array}{l}\text { Toned } \\
\text { unknow } \\
\text { colour }\end{array}$ \\
\hline 426012 & $\begin{array}{c}\text { Graphic } \\
\text { Consultants } \\
\text { LTD. } \\
\end{array}$ & 1972-0105 & $\begin{array}{c}\text { Canadian } \\
\text { Sea Fowl } \\
\text { No. } 3 \\
\end{array}$ & $\begin{array}{c}\text { The American } \\
\text { Eider Duck }\end{array}$ & $1933 ?$ & $\mathrm{cn}$ & CGMPB & $\begin{array}{l}\text { National Parks } \\
\text { of Canada, } \\
\text { Department of } \\
\text { Mines and } \\
\text { Resources } \\
\end{array}$ & OMPB & $\begin{array}{l}\text { This is a } \\
\text { bilingual } \\
\text { version }\end{array}$ \\
\hline 426591 & $\begin{array}{c}\text { Graphic } \\
\text { Consultants } \\
\text { LTD. } \\
\end{array}$ & 1972-0105 & $\begin{array}{c}\text { Canadian } \\
\text { Sea Fowl } \\
\text { No. } 4 \\
\end{array}$ & Fleet Wings & $1926 ?$ & $\mathrm{cn}$ & CGMPB & $\begin{array}{l}\text { National Parks } \\
\text { of Canada, } \\
\text { Department of } \\
\text { Mines and } \\
\text { Resources } \\
\end{array}$ & & Tinted Sepia \\
\hline 426793 & $\begin{array}{l}\text { Graphic } \\
\text { Consultants } \\
\text { LTD. } \\
\end{array}$ & 1972-0105 & & Ice : [incomplete] & 1920/1923? & $\mathrm{cn}$ & $\begin{array}{l}\text { Pathescope of } \\
\text { Canada }\end{array}$ & OMPB & & \\
\hline 431504 & $\begin{array}{l}\text { Graphic } \\
\text { Consultants } \\
\text { LTD. } \\
\end{array}$ & 1972-0105 & & $\begin{array}{c}\text { Trinidad and } \\
\text { Tobago at } \\
\text { Canadian National } \\
\text { Exhibition } \\
\end{array}$ & $1928 ?$ & $\mathrm{cn}$ & OMPB & & & \\
\hline 431507 & $\begin{array}{l}\text { Graphic } \\
\text { Consultants } \\
\text { LTD. } \\
\end{array}$ & 1972-0105 & & $\begin{array}{l}\text { [Trinidad : } \\
\text { incomplete] }\end{array}$ & 1920/1930? & N/A & N/A & & & \\
\hline 432155 & $\begin{array}{c}\text { Graphic } \\
\text { Consultants } \\
\text { LTD. } \\
\end{array}$ & 1972-0105 & $\begin{array}{l}\text { Canadian } \\
\text { Sea Fowl } \\
\end{array}$ & $\begin{array}{c}\text { The North Shore = } \\
\text { Le Côte-Nord }\end{array}$ & 1922 & $\mathrm{cn}$ & CGMPB & $\begin{array}{l}\text { National Parks } \\
\text { of Canada, } \\
\text { Department of } \\
\text { Mines and } \\
\text { Resources } \\
\end{array}$ & & \\
\hline 432193 & $\begin{array}{l}\text { Graphic } \\
\text { Consultants } \\
\text { LTD. } \\
\end{array}$ & 1972-0105 & & $\begin{array}{l}\text { Coconuts in } \\
\text { Trinidad }\end{array}$ & 1920/1925? & $\operatorname{tr}$ & $\begin{array}{c}\text { Tucker Picture } \\
\text { Company of } \\
\text { Trinidad; } \\
\text { Permanent } \\
\text { Exhibition } \\
\text { Committee } \\
\end{array}$ & & & \\
\hline 433043 & $\begin{array}{l}\text { Graphic } \\
\text { Consultants } \\
\text { LTD. } \\
\end{array}$ & $1972-0105$ & & $\begin{array}{c}\text { The Ancient and } \\
\text { Honourable } \\
\text { Walnut }\end{array}$ & 1920/1925? & us & $\begin{array}{c}\text { Bray } \\
\text { Productions Inc. }\end{array}$ & & & $\begin{array}{l}\text { Tinted } \\
\text { Orange } \\
\end{array}$ \\
\hline 433045 & $\begin{array}{l}\text { Graphic } \\
\text { Consultants } \\
\text { LTD. } \\
\end{array}$ & 1972-0105 & & $\begin{array}{c}\text { Concrete on the } \\
\text { Farm = Cement on } \\
\text { the Farm }\end{array}$ & 1920/1930? & $\mathrm{cn}$ & OMPB & $\begin{array}{c}\text { Canada Cement } \\
\text { Company } \\
\text { Limited } \\
\end{array}$ & & \\
\hline 433287 & $\begin{array}{c}\text { Graphic } \\
\text { Consultants } \\
\text { LTD. } \\
\end{array}$ & $1972-0105$ & & $\begin{array}{c}\text { Coast Salish } \\
\text { Indians of British } \\
\text { Columbia } \\
\end{array}$ & 1928 & $\mathrm{cn}$ & $\begin{array}{c}\text { National } \\
\text { Museum of } \\
\text { Canada }\end{array}$ & & OMPB & $\begin{array}{c}\text { Harlan } \\
\text { Ingersoll } \\
\text { Smith } \\
(1982-1940) \\
\end{array}$ \\
\hline
\end{tabular}




\begin{tabular}{|c|c|c|c|c|c|c|c|c|c|c|}
\hline 433972 & $\begin{array}{c} \\
\text { Graphic } \\
\text { Consultants } \\
\text { LTD. } \\
\end{array}$ & $1972-0105$ & & $\begin{array}{l}\text { With the Plowmen } \\
\text { at Brampton : } \\
\text { [incomplete] }\end{array}$ & $1924 / 1925$ & $\mathrm{cn}$ & OMPB & & & $\begin{array}{c}\text { The } \\
\text { archivist } \\
\text { notes, "One } \\
\text { of the most } \\
\text { interesting } \\
\text { features was } \\
\text { the large } \\
\text { numbers of } \\
\text { Indian } \\
\text { Farmers } \\
\text { present, } \\
\text { many of } \\
\text { whom were } \\
\text { prize } \\
\text { winners." }\end{array}$ \\
\hline 434210 & $\begin{array}{c}\text { Graphic } \\
\text { Consultants } \\
\text { LTD. } \\
\end{array}$ & $1972-0105$ & & Kegon Waterfall & 1920/1925? & $\mathrm{fr}$ & Pathe Freres & & OMPB & \\
\hline 434213 & $\begin{array}{c}\text { Graphic } \\
\text { Consultants } \\
\text { LTD. } \\
\end{array}$ & $1972-0105$ & & $\begin{array}{l}\text { Singapore : The } \\
\text { Gateway of the } \\
\text { East : [incomplete] }\end{array}$ & 1920/1925? & $\mathrm{fr}$ & Pathe Freres & & OMPB & \\
\hline 434214 & $\begin{array}{c}\text { Graphic } \\
\text { Consultants } \\
\text { LTD. } \\
\end{array}$ & 1972-0105 & & $\begin{array}{c}\text { Cairns Australia's } \\
\text { Winter Paradise }\end{array}$ & $1920 / 1924 ?$ & at & N/A & & & \\
\hline 435335 & $\begin{array}{c}\text { Graphic } \\
\text { Consultants } \\
\text { LTD. } \\
\end{array}$ & $1972-0105$ & & Canoeing & 1929/1930? & $\mathrm{cn}$ & OMPB & & & \\
\hline 435341 & $\begin{array}{c}\text { Graphic } \\
\text { Consultants } \\
\text { LTD. } \\
\end{array}$ & 1972-0105 & & $\begin{array}{c}\text { Horses -- The } \\
\text { Story of an } \\
\text { Ontario Bred Colt }\end{array}$ & $1925 ?$ & $\mathrm{cn}$ & OMPB & & & \\
\hline 435342 & $\begin{array}{c}\text { Graphic } \\
\text { Consultants } \\
\text { LTD. } \\
\end{array}$ & $1972-0105$ & & $\begin{array}{c}\text { Treasures of a } \\
\text { Great City : } \\
\text { History of the } \\
\text { Book } \\
\end{array}$ & 1918 & us & $\begin{array}{c}\text { Bray } \\
\text { Productions Inc. }\end{array}$ & & & $\begin{array}{l}\text { Tinted } \\
\text { unknown } \\
\text { colour } \\
\end{array}$ \\
\hline 435343 & $\begin{array}{c}\text { Graphic } \\
\text { Consultants } \\
\text { LTD. }\end{array}$ & $1972-0105$ & & $\begin{array}{l}\text { The Falls of } \\
\text { Monasterio }\end{array}$ & 1914/1924? & $\mathrm{fr}$ & Pathe Freres & & & \\
\hline 435344 & $\begin{array}{c}\text { Graphic } \\
\text { Consultants } \\
\text { LTD. } \\
\end{array}$ & $1972-0105$ & & $\begin{array}{c}\text { Sugar From Beet } \\
\text { to Bowl : } \\
\text { [incomplete] }\end{array}$ & 1915/1925? & $\mathrm{cn}$ & $\begin{array}{c}\text { Pathescope of } \\
\text { Canada }\end{array}$ & N/A & & $\begin{array}{l}\text { According } \\
\text { to archive } \\
\text { notes, this } \\
\text { was not } \\
\text { made by } \\
\text { OMPB }\end{array}$ \\
\hline 436241 & $\begin{array}{c}\text { Graphic } \\
\text { Consultants } \\
\text { LTD. } \\
\end{array}$ & $1972-0105$ & $\begin{array}{l}\text { Canadian } \\
\text { Sea Fowl } \\
\end{array}$ & $\begin{array}{c}\text { Nomads of the } \\
\text { Ocean = Nomades } \\
\text { de l'Ocean }\end{array}$ & $1928 ?$ & $\mathrm{cn}$ & CGMPB & $\begin{array}{c}\text { Canadian } \\
\text { National Parks } \\
\text { Branch } \\
\end{array}$ & & $\begin{array}{c}\text { Toned } \\
\text { unknown } \\
\text { colour } \\
\end{array}$ \\
\hline 437633 & $\begin{array}{c}\text { Graphic } \\
\text { Consultants } \\
\text { LTD. } \\
\end{array}$ & $1972-0105$ & $\begin{array}{c}\text { Paramount } \\
\text { Pictographs. } \\
\text { Better } \\
\text { Babies } \\
\end{array}$ & Body Building & 1920/1927? & us & $\begin{array}{c}\text { Paramount; } \\
\text { Women's Home } \\
\text { Companion } \\
\end{array}$ & & & $\begin{array}{l}\text { Tinted } \\
\text { unknown } \\
\text { colour } \\
\end{array}$ \\
\hline 438432 & $\begin{array}{c}\text { Graphic } \\
\text { Consultants } \\
\text { LTD. } \\
\end{array}$ & $1972-0105$ & & $\begin{array}{c}\text { The Arrival of } \\
\text { Santa From the } \\
\text { North }\end{array}$ & 1932 & $\mathrm{cn}$ & OMPB & & & \\
\hline 438617 & $\begin{array}{c}\text { Graphic } \\
\text { Consultants } \\
\text { LTD. } \\
\end{array}$ & $1972-0105$ & & $\begin{array}{c}\text { The Region of } \\
\text { Romance }\end{array}$ & $1920 ?$ & $\mathrm{cn}$ & $\begin{array}{c}\text { Exhibits and } \\
\text { Publicity Bureau }\end{array}$ & & & $\begin{array}{l}\text { Tinted } \\
\text { yellow }\end{array}$ \\
\hline 438659 & $\begin{array}{c}\text { Graphic } \\
\text { Consultants } \\
\text { LTD. } \\
\end{array}$ & 1972-0105 & & Trinidad Sugar & 1920/1924? & $\mathrm{cn}$ & OMPB & & OMPB & \\
\hline 438819 & $\begin{array}{c}\text { Graphic } \\
\text { Consultants } \\
\text { LTD. } \\
\end{array}$ & $1972-0105$ & & $\begin{array}{c}\text { Burwash Medical: } \\
\text { incomplete }\end{array}$ & $1921 ?$ & $\mathrm{cn}$ & $\begin{array}{c}\text { Filmcraft } \\
\text { Industries Ltd. }\end{array}$ & OMPB & & $\begin{array}{l}\text { "for medical } \\
\text { associations } \\
\text { only" }\end{array}$ \\
\hline 439094 & $\begin{array}{c}\text { Graphic } \\
\text { Consultants } \\
\text { LTD. } \\
\end{array}$ & 1972-0105 & & Strasburg & 1920/1924? & $\mathrm{fr}$ & Pathe Freres & & & \\
\hline 439106 & $\begin{array}{c}\text { Graphic } \\
\text { Consultants } \\
\text { LTD. }\end{array}$ & $1972-0105$ & & $\begin{array}{l}\text { A Canadian } \\
\text { National } \\
\text { Exhibition } \\
\text { Regatta: }\end{array}$ & 1929/1930? & cn & OMPB & & & \\
\hline
\end{tabular}




\begin{tabular}{|c|c|c|c|c|c|c|c|c|c|c|}
\hline & & & & [incomplete] & & & & & & \\
\hline 439147 & $\begin{array}{c}\text { Graphic } \\
\text { Consultants } \\
\text { LTD. } \\
\end{array}$ & 1972-0105 & & $\begin{array}{l}\text { [The Frog] : } \\
\text { incomplete }\end{array}$ & $1920 / 1924 ?$ & N/A & Pathe Freres & OMPB & & $\begin{array}{c}\text { no country } \\
\text { of } \\
\text { production. }\end{array}$ \\
\hline 439154 & $\begin{array}{c}\text { Graphic } \\
\text { Consultants } \\
\text { LTD. }\end{array}$ & $1972-0105$ & & $\begin{array}{c}\text { London in War } \\
\text { Time and During } \\
\text { Mobilization }\end{array}$ & 1914/1918? & N/A & Pathe Freres & & & \\
\hline 439155 & $\begin{array}{c}\text { Graphic } \\
\text { Consultants } \\
\text { LTD. }\end{array}$ & $1972-0105$ & & $\begin{array}{l}\text { The British Army. } \\
\text { Saluting the } \\
\text { Colours }\end{array}$ & 1914/1918? & N/A & Pathe Freres & OMPB & & $\begin{array}{c}\text { no country } \\
\text { of } \\
\text { production. }\end{array}$ \\
\hline 439260 & $\begin{array}{c}\text { Graphic } \\
\text { Consultants } \\
\text { LTD. } \\
\end{array}$ & 1972-0105 & $\begin{array}{l}\text { Canadian } \\
\text { National } \\
\text { Pictorial } \\
\end{array}$ & $\mathrm{n} / \mathrm{a}$ & $1919 / 1920$ & $\mathrm{cn}$ & $\begin{array}{c}\text { Pathescope of } \\
\text { Canada }\end{array}$ & $\begin{array}{c}\text { Exhibits and } \\
\text { Publicity Bureau } \\
\end{array}$ & & $\begin{array}{c}\text { Newsreel. } \\
\text { Tinted } \\
\text { unknow } \\
\text { colour } \\
\end{array}$ \\
\hline 439281 & $\begin{array}{c}\text { Graphic } \\
\text { Consultants } \\
\text { LTD. }\end{array}$ & $1972-0105$ & & $\begin{array}{c}\text { Paris and Her } \\
\text { Monuments }\end{array}$ & 1920/1924? & $\mathrm{fr}$ & Pathe Freres & & & \\
\hline 439494 & $\begin{array}{c}\text { Graphic } \\
\text { Consultants } \\
\text { LTD. } \\
\end{array}$ & 1972-0105 & Lafflets & $\mathrm{n} / \mathrm{a}$ & $1922 ?$ & us & N/A & & & $\begin{array}{c}\text { notes } \\
\text { suggest } \\
\text { Walt } \\
\text { Disney? }\end{array}$ \\
\hline 439974 & $\begin{array}{c}\text { Graphic } \\
\text { Consultants } \\
\text { LTD. }\end{array}$ & $1972-0105$ & & [Tea Growing] & 1920/1924? & $\mathrm{cn}$ & $\begin{array}{c}\text { Pathescope of } \\
\text { Canada }\end{array}$ & OMPB & & \\
\hline 440012 & $\begin{array}{c}\text { Graphic } \\
\text { Consultants } \\
\text { LTD. }\end{array}$ & $1972-0105$ & & $\begin{array}{c}\text { [Lake of Bays] : } \\
\text { incomplete }\end{array}$ & $1922 ?$ & $\mathrm{cn}$ & OMPB & & & $\begin{array}{l}\text { Tinted } \\
\text { unknown } \\
\text { colour } \\
\end{array}$ \\
\hline 440045 & $\begin{array}{c}\text { Graphic } \\
\text { Consultants } \\
\text { LTD. } \\
\end{array}$ & $1972-0105$ & & $\begin{array}{c}\text { [English city and } \\
\text { countryside] }\end{array}$ & 1930/1931 & $\mathrm{cn}$ & OMPB & & & \\
\hline 440605 & $\begin{array}{c}\text { Graphic } \\
\text { Consultants } \\
\text { LTD. }\end{array}$ & 1972-0105 & & $\begin{array}{l}\text { Angus and } \\
\text { Holsteins }\end{array}$ & $\mathrm{N} / \mathrm{A}$ & N/A & $\mathrm{N} / \mathrm{A}$ & & & \\
\hline 440607 & $\begin{array}{c}\text { Graphic } \\
\text { Consultants } \\
\text { LTD. }\end{array}$ & 1972-0105 & & $\begin{array}{c}\text { Clydesdale Mares } \\
\text { and Stallions at } \\
\text { International } \\
\text { Show } \\
\end{array}$ & & $\mathrm{cn}$ & N/A & & & $\begin{array}{c}\text { No } \\
\text { information } \\
\text { on this film }\end{array}$ \\
\hline 440608 & $\begin{array}{c}\text { Graphic } \\
\text { Consultants } \\
\text { LTD. }\end{array}$ & $1972-0105$ & & $\begin{array}{c}\text { [Egg and Poultry } \\
\text { Farming in } \\
\text { Saskatchewan] : } \\
\text { incomplete }\end{array}$ & 1920 & $\mathrm{cn}$ & $\mathrm{N} / \mathrm{A}$ & $\mathrm{N} / \mathrm{A}$ & & $\begin{array}{c}\text { No } \\
\text { information } \\
\text { on this film }\end{array}$ \\
\hline 440615 & $\begin{array}{c}\text { Graphic } \\
\text { Consultants } \\
\text { LTD. } \\
\end{array}$ & 1972-0105 & & $\begin{array}{c}\text { London in } \\
\text { Wartime and } \\
\text { During } \\
\text { Mobilization } \\
\end{array}$ & & $\mathrm{N} / \mathrm{A}$ & $\mathrm{N} / \mathrm{A}$ & N/A & & $\begin{array}{c}\text { No } \\
\text { information } \\
\text { on this film } \\
\end{array}$ \\
\hline 440616 & $\begin{array}{c}\text { Graphic } \\
\text { Consultants } \\
\text { LTD. }\end{array}$ & $1972-0105$ & & $\begin{array}{c}\text { [Milk : Natural } \\
\text { Perfect Food] }\end{array}$ & $1920 ?$ & us & N/A & $\mathrm{N} / \mathrm{A}$ & & \\
\hline 440784 & $\begin{array}{c}\text { Graphic } \\
\text { Consultants } \\
\text { LTD. } \\
\end{array}$ & $1972-0105$ & & $\begin{array}{c}\text { Mining Ontario } \\
\text { Graphite }= \\
\text { Graphite Mining }\end{array}$ & $1925 ?$ & $\mathrm{cn}$ & OMPB & & & \\
\hline 441176 & $\begin{array}{c}\text { Graphic } \\
\text { Consultants } \\
\text { LTD. }\end{array}$ & $1972-0105$ & & $\begin{array}{c}\text { The Construction } \\
\text { of a Concrete } \\
\text { Highway }\end{array}$ & 1917 & $\mathrm{cn}$ & $\begin{array}{c}\text { Pathescope of } \\
\text { Canada }\end{array}$ & OMPB & & \\
\hline 441342 & $\begin{array}{c}\text { Graphic } \\
\text { Consultants } \\
\text { LTD. } \\
\end{array}$ & $1972-0105$ & & $\begin{array}{c}\text { A Great Lakes } \\
\text { Romance }\end{array}$ & 1918 & $\mathrm{cn}$ & $\begin{array}{c}\text { Exhibits and } \\
\text { Publicity Bureau }\end{array}$ & $\begin{array}{l}\text { Department of } \\
\text { Trade and } \\
\text { Commerce } \\
\end{array}$ & OMPB & \\
\hline 441364 & $\begin{array}{c}\text { Graphic } \\
\text { Consultants } \\
\text { LTD. }\end{array}$ & $1972-0105$ & & $\begin{array}{c}\text { Native Life in } \\
\text { Borneo }\end{array}$ & 1920/1925? & N/A & Pathe Freres & & $\begin{array}{c}\text { Pathescope of } \\
\text { Canada }\end{array}$ & \\
\hline 443599 & $\begin{array}{c}\text { Graphic } \\
\text { Consultants } \\
\text { LTD. } \\
\end{array}$ & $1972-0105$ & & $\begin{array}{c}\text { Multiplying } \\
\text { Ontario's Power } \\
\text { Resources: } \\
\text { [incomplete] }\end{array}$ & 1919/1920? & $\mathrm{cn}$ & $\begin{array}{c}\text { Pathescope of } \\
\text { Canada }\end{array}$ & OMPB & & \\
\hline 443617 & $\begin{array}{c}\text { Graphic } \\
\text { Consultants } \\
\text { LTD. }\end{array}$ & $1972-0105$ & & $\begin{array}{c}\text { Queenston- } \\
\text { Chippawa } \\
\text { Development of }\end{array}$ & $1921 ?$ & $\mathrm{cn}$ & $\begin{array}{l}\text { Pathescope of } \\
\text { Canada }\end{array}$ & OMPB & & $\begin{array}{l}\text { Tinted } \\
\text { unknown } \\
\text { colour }\end{array}$ \\
\hline
\end{tabular}




\begin{tabular}{|c|c|c|c|c|c|c|c|c|c|c|}
\hline & & & & $\begin{array}{c}\text { the Hydro Electric } \\
\text { Power } \\
\text { Commission of } \\
\text { Ontario : } \\
\text { [incomplete] } \\
\end{array}$ & & & & & & \\
\hline 443655 & $\begin{array}{c}\text { Graphic } \\
\text { Consultants } \\
\text { LTD. }\end{array}$ & $1972-0105$ & & $\begin{array}{c}\text { The Chippawa } \\
\text { Canal }\end{array}$ & $1921 ?$ & $\mathrm{cn}$ & $\begin{array}{l}\text { Pathescope of } \\
\text { Canada }\end{array}$ & OMPB & & $\begin{array}{l}\text { Tinted } \\
\text { unknown } \\
\text { colour }\end{array}$ \\
\hline 443660 & $\begin{array}{c}\text { Graphic } \\
\text { Consultants } \\
\text { LTD. } \\
\end{array}$ & 1972-0105 & & $\begin{array}{c}\text { In the Ontario } \\
\text { Hydro Power } \\
\text { House at } \\
\text { Queenston } \\
\end{array}$ & $1920 ?$ & $\mathrm{cn}$ & $\begin{array}{c}\text { Pathescope of } \\
\text { Canada }\end{array}$ & OMPB & & $\begin{array}{l}\text { Tinted } \\
\text { unknown } \\
\text { colour } \\
\end{array}$ \\
\hline 443732 & $\begin{array}{c}\text { Graphic } \\
\text { Consultants } \\
\text { LTD. } \\
\end{array}$ & $1972-0105$ & $\begin{array}{c}\text { The Exploits } \\
\text { of Elaine }\end{array}$ & $\begin{array}{c}\text { Episode } 9 \text { The } \\
\text { Death Ray }\end{array}$ & $1914 / 1915$ & us & $\begin{array}{l}\text { Eclectic-Pathe } \\
\text { Exchange }\end{array}$ & & & \\
\hline 443733 & $\begin{array}{c}\text { Graphic } \\
\text { Consultants } \\
\text { LTD. }\end{array}$ & $1972-0105$ & $\begin{array}{c}\text { The Exploits } \\
\text { of Elaine }\end{array}$ & $\begin{array}{l}\text { Episode } 11 \text { The } \\
\text { Hour Of Three }\end{array}$ & $1914 / 1915$ & us & $\begin{array}{l}\text { Eclectic-Pathe } \\
\text { Exchange }\end{array}$ & & & \\
\hline 443736 & $\begin{array}{c}\text { Graphic } \\
\text { Consultants } \\
\text { LTD. }\end{array}$ & 1972-0105 & $\begin{array}{c}\text { The Exploits } \\
\text { of Elaine }\end{array}$ & $\begin{array}{l}\text { Episode } 10 \text { The } \\
\text { Life Current }\end{array}$ & $1914 / 1915$ & us & $\begin{array}{l}\text { Eclectic-Pathe } \\
\text { Exchange }\end{array}$ & & & \\
\hline 444812 & $\begin{array}{c}\text { Graphic } \\
\text { Consultants } \\
\text { LTD. } \\
\end{array}$ & 1972-0105 & & The Nest & 1927 & us & $\begin{array}{c}\text { Excellent } \\
\text { Pictures }\end{array}$ & & & \\
\hline 445085 & $\begin{array}{c}\text { Graphic } \\
\text { Consultants } \\
\text { LTD. }\end{array}$ & 1972-0105 & & [Team Rowing] & $1920 / 1930$ & $\mathrm{cn}$ & $\mathrm{N} / \mathrm{A}$ & $\mathrm{N} / \mathrm{A}$ & & $\begin{array}{l}\text { Unidentified } \\
\text { film footage }\end{array}$ \\
\hline 445655 & $\begin{array}{c}\text { Graphic } \\
\text { Consultants } \\
\text { LTD. } \\
\end{array}$ & 1972-0105 & & $\begin{array}{c}\text { [Scarborough Golf } \\
\text { Course : } \\
\text { incomplete] } \\
\end{array}$ & 1920/1929? & $\mathrm{cn}$ & $\mathrm{N} / \mathrm{A}$ & N/A & & $\begin{array}{c}\text { Unidentified } \\
\text { film } \\
\text { footage; } \\
\text { Scarborough } \\
\text { Golf and } \\
\text { Country } \\
\text { Club near } \\
\text { Toronto } \\
\end{array}$ \\
\hline 447036 & $\begin{array}{c}\text { Graphic } \\
\text { Consultants } \\
\text { LTD. } \\
\end{array}$ & 1972-0105 & & $\begin{array}{c}\text { Gathering and } \\
\text { Preparing } \\
\text { Cocoanuts in the } \\
\text { Philippines } \\
\end{array}$ & 1920/1929? & $\mathrm{fr}$ & Pathe Freres & & & \\
\hline 447653 & $\begin{array}{c}\text { Graphic } \\
\text { Consultants } \\
\text { LTD. }\end{array}$ & 1972-0105 & & The Cuttlefish & 1920/1924? & N/A & Pathe Freres & & & \\
\hline 458575 & $\begin{array}{c}\text { Graphic } \\
\text { Consultants } \\
\text { LTD. }\end{array}$ & 1972-0105 & $\begin{array}{c}\text { Pathé } \\
\text { Review } 26\end{array}$ & $\begin{array}{c}\text { Deep Water } \\
\text { Devils - Getting } \\
\text { Intimate with the } \\
\text { Octopus : } \\
\text { [incomplete] }\end{array}$ & 1919/1927? & us & Pathe Freres & & & \\
\hline 458581 & $\begin{array}{c}\text { Graphic } \\
\text { Consultants } \\
\text { LTD. } \\
\end{array}$ & 1972-0105 & & Plant it With Pines & $1929 ?$ & $\mathrm{cn}$ & OMPB & & & \\
\hline 459070 & $\begin{array}{c}\text { Graphic } \\
\text { Consultants } \\
\text { LTD. }\end{array}$ & $1972-0105$ & & $\begin{array}{l}\text { Concrete Tile } \\
\text { Manufacture }\end{array}$ & 1925/1926? & $\mathrm{cn}$ & OMPB & $\begin{array}{c}\text { Farm Drainage } \\
\text { Department } \\
\text { O.A.C., Guelph }\end{array}$ & & $\begin{array}{c}\text { Tinted } \\
\text { unknown } \\
\text { colour } \\
\end{array}$ \\
\hline 459111 & $\begin{array}{c}\text { Graphic } \\
\text { Consultants } \\
\text { LTD. }\end{array}$ & 1972-0105 & $\begin{array}{l}\text { Something } \\
\text { About } \\
\text { Australia } \\
\end{array}$ & Mountain Pastures & & at & $\begin{array}{c}\text { Government of } \\
\text { the } \\
\text { Commonwealth } \\
\text { of Australia }\end{array}$ & & & \\
\hline 459113 & $\begin{array}{c}\text { Graphic } \\
\text { Consultants } \\
\text { LTD. } \\
\end{array}$ & 1972-0105 & $\begin{array}{l}\text { Popular } \\
\text { Science } \\
\text { Films? }\end{array}$ & Australia & $1920 / 1929$ & at & $\begin{array}{c}\text { Scientific } \\
\text { Educational } \\
\text { Corporation } \\
\end{array}$ & & & $\begin{array}{l}\text { Tinted } \\
\text { unknown } \\
\text { colour } \\
\end{array}$ \\
\hline 459142 & $\begin{array}{c}\text { Graphic } \\
\text { Consultants } \\
\text { LTD. } \\
\end{array}$ & 1972-0105 & & $\begin{array}{c}\text { Professor Rouff's } \\
\text { Poison : } \\
\text { [incomplete] } \\
\end{array}$ & $1917 ?$ & fr & Pathe Freres & & OMPB & \\
\hline 460294 & $\begin{array}{c}\text { Graphic } \\
\text { Consultants } \\
\text { LTD. }\end{array}$ & $1972-0105$ & $\begin{array}{c}\text { Hepworth Q- } \\
\text { riosity by } \\
\text { "Q" }\end{array}$ & $\begin{array}{c}\text { If A Picture Tells } \\
\text { A Story }\end{array}$ & 1924 & uk & Hepworth & & OMPB & \\
\hline 460334 & $\begin{array}{c}\text { Graphic } \\
\text { Consultants } \\
\text { LTD. }\end{array}$ & 1972-0105 & & $\begin{array}{c}\text { Canadian National } \\
\text { Exhibition: } \\
\text { incomplete } \\
\end{array}$ & $1915 / 1920 ?$ & $\mathrm{cn}$ & $\begin{array}{c}\text { Pathescope of } \\
\text { Canada }\end{array}$ & OMPB & & \\
\hline
\end{tabular}




\begin{tabular}{|c|c|c|c|c|c|c|c|c|c|}
\hline 460338 & $\begin{array}{c}\text { Graphic } \\
\text { Consultants } \\
\text { LTD. }\end{array}$ & 1972-0105 & $\begin{array}{l}\text { Goldwyn- } \\
\text { Bray- } \\
\text { Pictograph }\end{array}$ & $\begin{array}{c}\text { The Movies } \\
\text { Exposed - Would } \\
\text { You Make A Date } \\
\text { With This Girl? } \\
\end{array}$ & 1920 & us & $\begin{array}{c}\text { Bray } \\
\text { Productions Inc. }\end{array}$ & & $\begin{array}{l}\text { Tinted } \\
\text { unknown } \\
\text { colour } \\
\end{array}$ \\
\hline 460432 & $\begin{array}{c}\text { Graphic } \\
\text { Consultants } \\
\text { LTD. } \\
\end{array}$ & $1972-0105$ & & $\begin{array}{c}\text { [Production of } \\
\text { Crushed Stone in } \\
\text { Ontario] }\end{array}$ & $1920 ?$ & cn & N/A & OMPB & $\begin{array}{c}\text { Remains } \\
\text { unidentified. } \\
\text { Tinted } \\
\text { yellow } \\
\end{array}$ \\
\hline 463273 & $\begin{array}{c}\text { Graphic } \\
\text { Consultants } \\
\text { LTD. }\end{array}$ & 1972-0105 & & $\begin{array}{c}\text { This Picture } \\
\text { Presented by the } \\
\text { Department of } \\
\text { Health of Ontario : } \\
\text { [presentation title] }\end{array}$ & $1920 / 1930$ & $\mathrm{cn}$ & OMPB & & \\
\hline 463353 & $\begin{array}{c}\text { Graphic } \\
\text { Consultants } \\
\text { LTD. }\end{array}$ & 1972-0105 & & $\begin{array}{l}\text { How the French } \\
\text { Wounded Soldiers } \\
\text { are Looked After } \\
=\text { How French } \\
\text { Wounded Soldiers } \\
\text { are Cared For } \\
\end{array}$ & & $\mathrm{fr}$ & Pathe Freres & & \\
\hline 463389 & $\begin{array}{c}\text { Graphic } \\
\text { Consultants } \\
\text { LTD. }\end{array}$ & $1972-0105$ & & $\begin{array}{l}\text { France's African } \\
\text { Cavalry on the } \\
\text { Banks of the Yser }\end{array}$ & 1914/1918? & fr & Pathe Freres & & \\
\hline 463390 & $\begin{array}{c}\text { Graphic } \\
\text { Consultants } \\
\text { LTD. } \\
\end{array}$ & $1972-0105$ & & $\begin{array}{c}\text { [Grenside For } \\
\text { Mayor] }\end{array}$ & 1920/1930? & $\mathrm{cn}$ & N/A & & $\begin{array}{c}\text { Home } \\
\text { movie, } \\
\text { might have } \\
\text { been shot by } \\
\text { George } \\
\text { Patton }\end{array}$ \\
\hline 463404 & $\begin{array}{c}\text { Graphic } \\
\text { Consultants } \\
\text { LTD. }\end{array}$ & 1972-0105 & & $\begin{array}{l}\text { [Unidentified } \\
\text { Women's } \\
\text { Assembly] } \\
\end{array}$ & $1920 / 1930 ?$ & $\mathrm{cn}$ & N/A & & $\begin{array}{l}\text { unknown } \\
\text { footage }\end{array}$ \\
\hline 463846 & $\begin{array}{c}\text { Graphic } \\
\text { Consultants } \\
\text { LTD. } \\
\end{array}$ & 1972-0105 & The Maori & As He Was & 1920/1929? & $\mathrm{nz}$ & $\begin{array}{c}\text { New Zealand } \\
\text { Government } \\
\text { Publicity Office }\end{array}$ & & \\
\hline 464107 & $\begin{array}{c}\text { Graphic } \\
\text { Consultants } \\
\text { LTD. }\end{array}$ & $1972-0105$ & & $\begin{array}{l}\text { Adam Bede: } \\
\text { [incomplete] }\end{array}$ & $1915 ?$ & us & N/A & & $\begin{array}{c}\text { lacking } \\
\text { information }\end{array}$ \\
\hline 464389 & $\begin{array}{c}\text { Graphic } \\
\text { Consultants } \\
\text { LTD. } \\
\end{array}$ & $1972-0105$ & & $\begin{array}{c}\text { [Beef Cattle in } \\
\text { Ontario] }\end{array}$ & 1920/1929? & $\mathrm{cn}$ & OMPB & & unidentified \\
\hline 464705 & $\begin{array}{c}\text { Graphic } \\
\text { Consultants } \\
\text { LTD. } \\
\end{array}$ & $1972-0105$ & $\begin{array}{c}\text { Hey Fellas! } \\
\text { Comedies }\end{array}$ & The Klynick & $1925 ?$ & us & $\begin{array}{l}\text { J. Charles Davis; } \\
\text { A McKnight } \\
\text { Womack } \\
\text { Production }\end{array}$ & $\begin{array}{c}\text { Davis } \\
\text { Distributing } \\
\text { Division Inc. }\end{array}$ & \\
\hline 466381 & $\begin{array}{c}\text { Graphic } \\
\text { Consultants } \\
\text { LTD. }\end{array}$ & $1972-0105$ & $\begin{array}{l}\text { The Exploits } \\
\text { of Elaine }\end{array}$ & $\begin{array}{l}\text { Episode } 2 \text { The } \\
\text { Twilight Sleep }\end{array}$ & $1914 / 1915$ & us & $\begin{array}{l}\text { Eclectic-Pathe } \\
\text { Exchange }\end{array}$ & & \\
\hline 466382 & $\begin{array}{c}\text { Graphic } \\
\text { Consultants } \\
\text { LTD. }\end{array}$ & $1972-0105$ & $\begin{array}{l}\text { The Exploits } \\
\text { of Elaine }\end{array}$ & $\begin{array}{l}\text { Episode } 3 \text { The } \\
\text { Vanishing Jewels }\end{array}$ & $1914 / 1915$ & us & $\begin{array}{l}\text { Eclectic-Pathe } \\
\text { Exchange }\end{array}$ & & \\
\hline 466383 & $\begin{array}{c}\text { Graphic } \\
\text { Consultants } \\
\text { LTD. }\end{array}$ & $1972-0105$ & $\begin{array}{l}\text { The Exploits } \\
\text { of Elaine }\end{array}$ & $\begin{array}{l}\text { Episode } 4 \text { The } \\
\text { Frozen Safe : } \\
\text { [incomplete] }\end{array}$ & $1914 / 1915$ & us & $\begin{array}{l}\text { Eclectic-Pathe } \\
\text { Exchange }\end{array}$ & & \\
\hline 466384 & $\begin{array}{c}\text { Graphic } \\
\text { Consultants } \\
\text { LTD. }\end{array}$ & $1972-0105$ & $\begin{array}{l}\text { The Exploits } \\
\text { of Elaine }\end{array}$ & $\begin{array}{c}\text { Episode } 5 \text { The } \\
\text { Poisoned Room : } \\
\text { [incomplete] }\end{array}$ & $1914 / 1915$ & us & $\begin{array}{l}\text { Eclectic-Pathe } \\
\text { Exchange }\end{array}$ & & \\
\hline 466385 & $\begin{array}{c}\text { Graphic } \\
\text { Consultants } \\
\text { LTD. } \\
\end{array}$ & $1972-0105$ & $\begin{array}{l}\text { The Exploits } \\
\text { of Elaine }\end{array}$ & $\begin{array}{c}\text { Episode } 6 \text { The } \\
\text { Vampire: } \\
\text { [incomplete] }\end{array}$ & $1914 / 1915$ & us & $\begin{array}{l}\text { Eclectic-Pathe } \\
\text { Exchange }\end{array}$ & & \\
\hline 466389 & $\begin{array}{c}\text { Graphic } \\
\text { Consultants } \\
\text { LTD. }\end{array}$ & $1972-0105$ & $\begin{array}{l}\text { The Exploits } \\
\text { of Elaine }\end{array}$ & $\begin{array}{c}\text { Episode } 7 \text { The } \\
\text { Double Trap }\end{array}$ & $1914 / 1915$ & us & $\begin{array}{l}\text { Eclectic-Pathe } \\
\text { Exchange }\end{array}$ & & \\
\hline 466393 & $\begin{array}{c}\text { Graphic } \\
\text { Consultants } \\
\text { LTD. } \\
\end{array}$ & 1972-0105 & $\begin{array}{l}\text { The Exploits } \\
\text { of Elaine }\end{array}$ & $\begin{array}{c}\text { Episode } 8 \text { The } \\
\text { Hidden Voice }\end{array}$ & $1914 / 1915$ & us & $\begin{array}{l}\text { Eclectic-Pathe } \\
\text { Exchange }\end{array}$ & & \\
\hline 466419 & $\begin{array}{c}\text { Graphic } \\
\text { Consultants } \\
\text { LTD. }\end{array}$ & $1972-0105$ & $\begin{array}{l}\text { The Exploits } \\
\text { of Elaine }\end{array}$ & $\begin{array}{l}\text { Episode } 12 \text { The } \\
\text { Blood Crystals }\end{array}$ & $1914 / 1915$ & us & $\begin{array}{l}\text { Eclectic-Pathe } \\
\text { Exchange }\end{array}$ & & \\
\hline
\end{tabular}




\begin{tabular}{|c|c|c|c|c|c|c|c|c|c|c|}
\hline 466424 & $\begin{array}{c}\text { Graphic } \\
\text { Consultants } \\
\text { LTD. }\end{array}$ & $1972-0105$ & $\begin{array}{l}\text { The Exploits } \\
\text { of Elaine }\end{array}$ & $\begin{array}{c}\text { Episode } 13 \text { The } \\
\text { Devil Worshippers }\end{array}$ & $1914 / 1915$ & us & $\begin{array}{l}\text { Eclectic-Pathe } \\
\text { Exchange }\end{array}$ & & & \\
\hline 466430 & $\begin{array}{c}\text { Graphic } \\
\text { Consultants } \\
\text { LTD. } \\
\end{array}$ & $1972-0105$ & $\begin{array}{l}\text { The Exploits } \\
\text { of Elaine }\end{array}$ & $\begin{array}{c}\text { Episode } 14 \text { The } \\
\text { Reckoning }\end{array}$ & $1914 / 1915$ & us & $\begin{array}{l}\text { Eclectic-Pathe } \\
\text { Exchange }\end{array}$ & & & \\
\hline 466441 & $\begin{array}{c}\text { Graphic } \\
\text { Consultants } \\
\text { LTD. } \\
\end{array}$ & $1972-0105$ & & $\begin{array}{c}\text { The Chosen Prince } \\
\text { or The Friendship } \\
\text { of David and } \\
\text { Jonathan }\end{array}$ & 1917 & us & $\begin{array}{l}\text { The Crest } \\
\text { Picture } \\
\text { Company }\end{array}$ & & & $\begin{array}{l}\text { Tinted } \\
\text { unknown } \\
\text { colour }\end{array}$ \\
\hline 466487 & $\begin{array}{c}\text { Graphic } \\
\text { Consultants } \\
\text { LTD. } \\
\end{array}$ & $1972-0105$ & & Pondichery & 1920/1924? & $\mathrm{fr}$ & Pathe Freres & & & \\
\hline 466518 & $\begin{array}{c}\text { Graphic } \\
\text { Consultants } \\
\text { LTD. }\end{array}$ & $1972-0105$ & & $\begin{array}{l}\text { Native Festival } \\
\text { Near Pondichery } \\
\text { (India) }\end{array}$ & 1920/1924? & $\mathrm{fr}$ & Pathe Freres & & & \\
\hline 467001 & $\begin{array}{c}\text { Graphic } \\
\text { Consultants } \\
\text { LTD. } \\
\end{array}$ & $1972-0105$ & $\begin{array}{c}\text { Oriental } \\
\text { Cruise Series } \\
\end{array}$ & $\begin{array}{c}\text { Singapore: } \\
\text { [incomplete] }\end{array}$ & 1920/1930? & $\mathrm{cn}$ & OMPB & & OMPB & $\begin{array}{c}\text { Might be } \\
\text { part of } \\
\text { OMPB } \\
\text { Oriental } \\
\text { Cruise } \\
\text { Series } \\
\end{array}$ \\
\hline 468867 & $\begin{array}{c}\text { Graphic } \\
\text { Consultants } \\
\text { LTD. }\end{array}$ & $1972-0105$ & & $\begin{array}{c}\text { [Unidentified } \\
\text { Charlie Chaplin] : } \\
\text { [excerpt] }\end{array}$ & $1916 ?$ & us & N/A & & OMPB & $\begin{array}{l}\text { Tinted } \\
\text { yellow }\end{array}$ \\
\hline 473395 & $\begin{array}{c}\text { Graphic } \\
\text { Consultants } \\
\text { LTD. }\end{array}$ & $1972-0105$ & & $\begin{array}{c}\text { Manufacture of } \\
\text { Bamboo Hats }\end{array}$ & $1920 / 1922$ & N/A & Pathe Freres & & & \\
\hline 473464 & $\begin{array}{c}\text { Graphic } \\
\text { Consultants } \\
\text { LTD. }\end{array}$ & $1972-0105$ & $\begin{array}{c}\text { Daypho- } \\
\text { Bray } \\
\text { Pictograph } \\
\end{array}$ & $\begin{array}{c}\text { Putting Volcanoes } \\
\text { to Work }\end{array}$ & 1917 & us & $\begin{array}{c}\text { Bray } \\
\text { Productions Inc. }\end{array}$ & $\begin{array}{c}\text { Popular Science } \\
\text { Weekly }\end{array}$ & & \\
\hline 473468 & $\begin{array}{c}\text { Graphic } \\
\text { Consultants } \\
\text { LTD. } \\
\end{array}$ & $1972-0105$ & & $\begin{array}{l}\text { The Beaver's } \\
\text { Storehouse }\end{array}$ & N/A & us & Pathe Review & & & \\
\hline 473473 & $\begin{array}{c}\text { Graphic } \\
\text { Consultants } \\
\text { LTD. } \\
\end{array}$ & $1972-0105$ & & $\begin{array}{l}\text { [Trinidad] - } \\
\text { incomplete }\end{array}$ & N/A & N/A & N/A & & OMPB & \\
\hline 476709 & $\begin{array}{c}\text { Graphic } \\
\text { Consultants } \\
\text { LTD. }\end{array}$ & $1972-0105$ & & $\begin{array}{c}\text { General Joffre in } \\
\text { Alsace : } \\
\text { incomplete }\end{array}$ & $\mathrm{N} / \mathrm{A}$ & $\mathrm{fr}$ & Pathe Freres & & & \\
\hline 528089 & $\begin{array}{c}\text { Graphic } \\
\text { Consultants } \\
\text { LTD. }\end{array}$ & $1972-0105$ & & $\begin{array}{l}\text { Excursion on the } \\
\text { Mekong = An } \\
\text { Excursion to the } \\
\text { Mekong (Indo- } \\
\text { China) }\end{array}$ & $\mathrm{N} / \mathrm{A}$ & $\mathrm{fr}$ & Pathe Freres & & & \\
\hline 528366 & $\begin{array}{c}\text { Graphic } \\
\text { Consultants } \\
\text { LTD. }\end{array}$ & $1972-0105$ & & Ice in the Baltic & 1920/1924? & $\mathrm{fr}$ & Pathe Freres & & & \\
\hline 528415 & $\begin{array}{c}\text { Graphic } \\
\text { Consultants } \\
\text { LTD. }\end{array}$ & $1972-0105$ & & $\begin{array}{l}\text { A Wedding in } \\
\text { Brittany }\end{array}$ & 1920/1929? & $\mathrm{fr}$ & Pathe Freres & & & \\
\hline 534344 & $\begin{array}{c}\text { Graphic } \\
\text { Consultants } \\
\text { LTD. }\end{array}$ & $1972-0105$ & & $\begin{array}{c}\text { A Wood Chopping } \\
\text { Contest in New } \\
\text { Zealand } \\
\end{array}$ & 1917 & us & $\begin{array}{c}\text { Bray } \\
\text { Productions Inc. }\end{array}$ & & & $\begin{array}{c}\text { Tinted } \\
\text { unknown } \\
\text { colour } \\
\end{array}$ \\
\hline 534409 & $\begin{array}{c}\text { Graphic } \\
\text { Consultants } \\
\text { LTD. } \\
\end{array}$ & $1972-0105$ & & $\begin{array}{l}\text { Gathering } \\
\text { Pineapples }\end{array}$ & 1920/1924? & $\mathrm{fr}$ & Pathe Freres & & & $\begin{array}{l}\text { Tinted } \\
\text { orange }\end{array}$ \\
\hline 534413 & $\begin{array}{c}\text { Graphic } \\
\text { Consultants } \\
\text { LTD. }\end{array}$ & $1972-0105$ & & $\begin{array}{l}\text { The Fastest } \\
\text { Fingers in } \\
\text { America }\end{array}$ & 1920/1930? & N/A & N/A & & & $\begin{array}{c}\text { Tinted } \\
\text { unknown } \\
\text { colour } \\
\end{array}$ \\
\hline 534720 & $\begin{array}{c}\text { Graphic } \\
\text { Consultants } \\
\text { LTD. }\end{array}$ & $1972-0105$ & & $\begin{array}{c}\text { Felix the Cat Gets } \\
\text { Revenge }\end{array}$ & 1922/1927? & us & $\begin{array}{c}\text { Pat Sullivan } \\
\text { Studio }\end{array}$ & & & $\begin{array}{l}\text { Tinted } \\
\text { unknown } \\
\text { colour }\end{array}$ \\
\hline 536778 & $\begin{array}{c}\text { Graphic } \\
\text { Consultants } \\
\text { LTD. }\end{array}$ & $1972-0105$ & $\begin{array}{l}\text { Pathé Freres' } \\
\text { Instructive } \\
\text { Series }\end{array}$ & $\begin{array}{l}\text { An Upside Down } \\
\text { Animal: The } \\
\text { Flying-Fox }\end{array}$ & 1920/1924? & fr & Pathe Freres & & & \\
\hline
\end{tabular}




\begin{tabular}{|c|c|c|c|c|c|c|c|c|c|c|}
\hline 538002 & $\begin{array}{c}\text { Graphic } \\
\text { Consultants } \\
\text { LTD. } \\
\end{array}$ & $1972-0105$ & & $\begin{array}{l}\text { [Alligator } \\
\text { Farming] }\end{array}$ & 1920/1924? & N/A & $\begin{array}{l}\text { Bray Studios } \\
\text { Inc. }\end{array}$ & & & $\begin{array}{l}\text { Tinted } \\
\text { unknown } \\
\text { colour } \\
\end{array}$ \\
\hline 539450 & $\begin{array}{c}\text { Graphic } \\
\text { Consultants } \\
\text { LTD. } \\
\end{array}$ & 1972-0105 & & Pep in Feathers & 1920/1930? & $\mathrm{fr}$ & Pathe Freres & & & \\
\hline 539451 & $\begin{array}{c}\text { Graphic } \\
\text { Consultants } \\
\text { LTD. } \\
\end{array}$ & 1972-0105 & & $\begin{array}{l}\text { Feathered } \\
\text { Celebrities }\end{array}$ & 1920/1930? & us & Pathe Review & & & \\
\hline 540177 & $\begin{array}{c}\text { Graphic } \\
\text { Consultants } \\
\text { LTD. } \\
\end{array}$ & $1972-0105$ & & Sand-Hoppers & $1920 / 1929 ?$ & $\mathrm{fr}$ & Pathe Freres & & & \\
\hline 540178 & $\begin{array}{c}\text { Graphic } \\
\text { Consultants } \\
\text { LTD. } \\
\end{array}$ & $1972-0105$ & & $\begin{array}{c}\text { Cockle-Gathering } \\
\text { on the Normandy } \\
\text { Coast = Shells }\end{array}$ & 1920/1929? & $\mathrm{fr}$ & Pathe Freres & & & \\
\hline 541265 & $\begin{array}{c}\text { Graphic } \\
\text { Consultants } \\
\text { LTD. } \\
\end{array}$ & $1972-0105$ & & $\begin{array}{c}\text { Wild Babies: } \\
\text { incomplete }\end{array}$ & 1921 & N/A & $\begin{array}{c}\text { Bray } \\
\text { Productions Inc. }\end{array}$ & & & $\begin{array}{l}\text { Tinted } \\
\text { unknown } \\
\text { colour } \\
\end{array}$ \\
\hline 556191 & $\begin{array}{l}\text { Graphic } \\
\text { Consultants } \\
\text { LTD. } \\
\end{array}$ & $1972-0105$ & $\begin{array}{l}\text { Gem of the } \\
\text { Screen }\end{array}$ & The Magic Hour & 1920/1924? & N/A & N/A & $\begin{array}{c}\text { Cranfield and } \\
\text { Clarke }\end{array}$ & OMPB & $\begin{array}{c}\text { no } \\
\text { description. } \\
\text { Tinted } \\
\text { unknown } \\
\text { colour } \\
\end{array}$ \\
\hline 565539 & $\begin{array}{l} \\
\text { Graphic } \\
\text { Consultants } \\
\text { LTD. } \\
\end{array}$ & 1972-0105 & & $\begin{array}{l}\text { Iron and Steel } \\
\text { From the Ore Bed } \\
\text { to the Smelter : } \\
\text { [incomplete] }\end{array}$ & $1917 / 1922$ & cn & $\begin{array}{l}\text { Pathescope of } \\
\text { Canada }\end{array}$ & OMPB & & $\begin{array}{c}\text { Tinted } \\
\text { intertitles } \\
\text { unknown } \\
\text { colour. } \\
\text { French and } \\
\text { English } \\
\text { intertitles } \\
\end{array}$ \\
\hline 566468 & $\begin{array}{c}\text { Graphic } \\
\text { Consultants } \\
\text { LTD. } \\
\end{array}$ & 1972-0105 & & $\begin{array}{c}\text { An Upside Down } \\
\text { Animal: The } \\
\text { Flying Fox } \\
\end{array}$ & $1920 / 1924 ?$ & $\mathrm{fr}$ & Pathe Freres & & & \\
\hline 3958 & $\begin{array}{c}\text { Graphic } \\
\text { Consultants } \\
\text { LTD. } \\
\end{array}$ & 1972-0105 & & $\begin{array}{l}\text { Prince Edward's } \\
\text { Industries }\end{array}$ & 1921 & $\mathrm{cn}$ & $\begin{array}{c}\text { Filmcraft } \\
\text { Industries Ltd. ; } \\
\text { OMPB } \\
\end{array}$ & & & \\
\hline 9479 & $\begin{array}{c}\text { Graphic } \\
\text { Consultants } \\
\text { LTD. } \\
\end{array}$ & $1972-0105$ & & $\begin{array}{c}\text { After One } \\
\text { Hundred Years : } \\
\text { [incomplete] }\end{array}$ & 1927 & $\mathrm{cn}$ & OMPB & & & \\
\hline 9481 & $\begin{array}{c}\text { Graphic } \\
\text { Consultants } \\
\text { LTD. } \\
\end{array}$ & 1972-0105 & & $\begin{array}{c}\text { Commencement } \\
\text { University of } \\
\text { Toronto } \\
\end{array}$ & 1923/1924? & $\mathrm{cn}$ & OMPB & & & \\
\hline 9483 & $\begin{array}{c}\text { Graphic } \\
\text { Consultants } \\
\text { LTD. } \\
\end{array}$ & 1972-0105 & & $\begin{array}{l}\text { The Ontario } \\
\text { Agricultural } \\
\text { College }\end{array}$ & $1926 / 1929$ & $\mathrm{cn}$ & OMPB & & & \\
\hline 9484 & $\begin{array}{c}\text { Graphic } \\
\text { Consultants } \\
\text { LTD. } \\
\end{array}$ & 1972-0105 & & $\begin{array}{c}\text { Royal } \\
\text { Remembrances }\end{array}$ & 1929 & uk & $\begin{array}{l}\text { The Gaumont } \\
\text { Company Ltd. }\end{array}$ & & & \\
\hline 11345 & $\begin{array}{c}\text { Graphic } \\
\text { Consultants } \\
\text { LTD. } \\
\end{array}$ & 1972-0105 & & $\begin{array}{c}\text { Glimpses of } \\
\text { London England }\end{array}$ & $1926 / 1928 ?$ & $\mathrm{uk}$ & N/A & $\begin{array}{c}\text { Cunard and } \\
\text { Anchor- } \\
\text { Donaldson Lines } \\
\end{array}$ & & \\
\hline 12261 & $\begin{array}{c}\text { Graphic } \\
\text { Consultants } \\
\text { LTD. }\end{array}$ & $1972-0105$ & & $\begin{array}{l}\text { The Royal } \\
\text { Canadian } \\
\text { Dragoons }\end{array}$ & $1920 / 1929$ & $\mathrm{cn}$ & $\begin{array}{l}\text { Pathescope of } \\
\text { Canada }\end{array}$ & & & \\
\hline 19700 & $\begin{array}{c}\text { Graphic } \\
\text { Consultants } \\
\text { LTD. } \\
\end{array}$ & $1972-0105$ & & $\begin{array}{c}\text { Queen's } \\
\text { University } 1841- \\
1926 \text { : Eighty Five } \\
\text { Years of Loyal } \\
\text { Service }\end{array}$ & 1926 & $\mathrm{cn}$ & OMPB & & & \\
\hline 21329 & $\begin{array}{l}\text { Graphic } \\
\text { Consultants } \\
\text { LTD. }\end{array}$ & $1972-0105$ & & $\begin{array}{c}\text { The Construction } \\
\text { of an Asphaltic- } \\
\text { Concrete Road } \\
\text { Surface } \\
\end{array}$ & 1917 & $\mathrm{cn}$ & OMPB & & & \\
\hline 24223 & $\begin{array}{c}\text { Graphic } \\
\text { Consultants } \\
\text { LTD. } \\
\end{array}$ & 1972-0105 & & Spare Time & 1927 & $\mathrm{cn}$ & OMPB & $\begin{array}{c}\text { Canadian } \\
\text { Forestry } \\
\text { Association } \\
\end{array}$ & & $\begin{array}{c}\text { no } \\
\text { description } \\
\end{array}$ \\
\hline 25246 & $\begin{array}{c}\text { Graphic } \\
\text { Consultants } \\
\text { LTD. } \\
\end{array}$ & 1972-0105 & & $\begin{array}{c}\text { Victory Loan } \\
\text { Parade with } \\
\text { British Tank and }\end{array}$ & 1918 & N/A & N/A & & & $\begin{array}{c}\text { no } \\
\text { description }\end{array}$ \\
\hline
\end{tabular}




\begin{tabular}{|c|c|c|c|c|c|c|c|c|c|c|}
\hline & & & & American Troops & & & & & & \\
\hline 25679 & $\begin{array}{l}\text { Graphic } \\
\text { Consultants } \\
\text { LTD. } \\
\end{array}$ & 1972-0105 & & $\begin{array}{c}\text { Cinderella of the } \\
\text { Farms }\end{array}$ & 1930 & $\mathrm{cn}$ & OMPB & & & \\
\hline 41858 & $\begin{array}{c}\text { Graphic } \\
\text { Consultants } \\
\text { LTD. } \\
\end{array}$ & 1972-0105 & & Current Gossip & $1922 ?$ & $\mathrm{cn}$ & $\begin{array}{c}\text { Filmcraft } \\
\text { Industries Ltd. }\end{array}$ & OMPB & & \\
\hline 43337 & $\begin{array}{l}\text { Graphic } \\
\text { Consultants } \\
\text { LTD. } \\
\end{array}$ & 1972-0105 & & $\begin{array}{c}\text { Butter Making in } \\
\text { New Ontario = } \\
\text { Butter Making in } \\
\text { Ontario } \\
\end{array}$ & $1918 / 1927$ & $\mathrm{cn}$ & OMPB & & & \\
\hline 47033 & $\begin{array}{l}\text { Graphic } \\
\text { Consultants } \\
\text { LTD. }\end{array}$ & 1972-0105 & & $\begin{array}{c}\text { Britain's Future } \\
\text { King }\end{array}$ & 1919 & $\mathrm{cn}$ & $\begin{array}{l}\text { Pathescope of } \\
\text { Canada }\end{array}$ & & OMPB & \\
\hline 52545 & $\begin{array}{c}\text { Graphic } \\
\text { Consultants } \\
\text { LTD. }\end{array}$ & $1972-0105$ & & Herring Fishing & $1920 / 1925$ & $\mathrm{cn}$ & Pathe & & OMPB & \\
\hline 53228 & $\begin{array}{c}\text { Graphic } \\
\text { Consultants } \\
\text { LTD. }\end{array}$ & $1972-0105$ & $\begin{array}{c}\text { Popular } \\
\text { Science } \\
\text { Films } \\
\end{array}$ & Earth and Moon & 1907/1930? & us & $\begin{array}{c}\text { Scientific } \\
\text { Educational } \\
\text { Corporation } \\
\end{array}$ & & OMPB & \\
\hline 54471 & $\begin{array}{l}\text { Graphic } \\
\text { Consultants } \\
\text { LTD. }\end{array}$ & $1972-0105$ & & $\begin{array}{c}\text { In Action With } \\
\text { Our Canadian } \\
\text { Troops : } \\
\text { [incomplete] }\end{array}$ & 1917 & uk & $\begin{array}{l}\text { Canadian War } \\
\text { Records Office }\end{array}$ & $\begin{array}{l}\text { War Office } \\
\text { Cinema } \\
\text { Committee }\end{array}$ & & \\
\hline 69391 & $\begin{array}{c}\text { Graphic } \\
\text { Consultants } \\
\text { LTD. } \\
\end{array}$ & $1972-0105$ & & $\begin{array}{c}\text { Round Przemysl = } \\
\text { [With the Russian } \\
\text { Army] }\end{array}$ & 1916 & $\mathrm{fr}$ & Pathe Freres & & & \\
\hline 72181 & $\begin{array}{c}\text { Graphic } \\
\text { Consultants } \\
\text { LTD. }\end{array}$ & $1972-0105$ & & $\begin{array}{l}\text { Gold Production } \\
\text { in Ontario }\end{array}$ & 1926 & $\mathrm{cn}$ & OMPB & & & \\
\hline 84866 & $\begin{array}{l}\text { Graphic } \\
\text { Consultants } \\
\text { LTD. } \\
\end{array}$ & 1972-0105 & & $\begin{array}{c}\text { The Unshod } \\
\text { Soldiers of a King }\end{array}$ & 1920 & us & $\begin{array}{c}\text { Goldwyn-Bray } \\
\text { Studios } \\
\end{array}$ & & & $\begin{array}{c}\text { Tinted } \\
\text { unknown } \\
\text { colour } \\
\end{array}$ \\
\hline 90568 & $\begin{array}{c}\text { Graphic } \\
\text { Consultants } \\
\text { LTD. } \\
\end{array}$ & $1972-0105$ & & Afloat in Muskoka & 1925 & $\mathrm{cn}$ & OMPB & & & \\
\hline 93327 & $\begin{array}{l}\text { Graphic } \\
\text { Consultants } \\
\text { LTD. }\end{array}$ & $1972-0105$ & & $\begin{array}{c}\text { General View of } \\
\text { the Grounds at the } \\
\text { Canadian National } \\
\text { Exhibition } \\
\text { Toronto }= \\
\text { [General View of } \\
\text { Cattle at CNE] }\end{array}$ & 1920 & $\mathrm{cn}$ & N/A & & & \\
\hline 93350 & $\begin{array}{l}\text { Graphic } \\
\text { Consultants } \\
\text { LTD. } \\
\end{array}$ & 1972-0105 & & $\begin{array}{c}\text { Ayrshires at the } \\
\text { Canadian National } \\
\text { Exhibition } \\
\text { Toronto } \\
\end{array}$ & 1922 & $\mathrm{cn}$ & $\begin{array}{c}\text { Pathescope of } \\
\text { Canada }\end{array}$ & OMPB & & \\
\hline 93351 & $\begin{array}{c}\text { Graphic } \\
\text { Consultants } \\
\text { LTD. }\end{array}$ & $1972-0105$ & & $\begin{array}{l}\text { Bituminous } \\
\text { Treatment of } \\
\text { Gravel Roads }\end{array}$ & $1926 ?$ & cn & OMPB & & & \\
\hline 93357 & $\begin{array}{c}\text { Graphic } \\
\text { Consultants } \\
\text { LTD. }\end{array}$ & $1972-0105$ & & $\begin{array}{c}\text { A School Fair in } \\
\text { Ontario }\end{array}$ & 1932 & $\mathrm{cn}$ & OMPB & & & \\
\hline 93372 & $\begin{array}{c}\text { Graphic } \\
\text { Consultants } \\
\text { LTD. }\end{array}$ & $1972-0105$ & & $\begin{array}{l}\text { Construction of an } \\
\text { Earth Road }\end{array}$ & $1920 / 1925$ & $\mathrm{cn}$ & OMPB & & & \\
\hline 93380 & $\begin{array}{c}\text { Graphic } \\
\text { Consultants } \\
\text { LTD. }\end{array}$ & 1972-0105 & & Royal Winter Fair & $1920 / 1925$ & cn & OMPB & & & \\
\hline 95343 & $\begin{array}{l}\text { Graphic } \\
\text { Consultants } \\
\text { LTD. } \\
\end{array}$ & $1972-0105$ & & 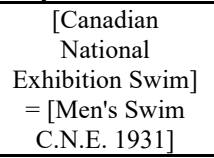 & 1931 & $\mathrm{cn}$ & OMPB & & & \\
\hline 99348 & $\begin{array}{l}\text { Graphic } \\
\text { Consultants } \\
\text { LTD. }\end{array}$ & $1972-0105$ & $\begin{array}{c}\text { Seeing } \\
\text { Canada } \\
\end{array}$ & $\begin{array}{c}\text { A Money Making } \\
\text { Industry }\end{array}$ & 1920 & $\mathrm{cn}$ & $\begin{array}{c}\text { Exhibits and } \\
\text { Publicity Bureau }\end{array}$ & & & \\
\hline
\end{tabular}




\begin{tabular}{|c|c|c|c|c|c|c|c|c|}
\hline 99688 & $\begin{array}{c}\text { Graphic } \\
\text { Consultants } \\
\text { LTD. } \\
\end{array}$ & 1972-0105 & Her Own Fault & 1921 & $\mathrm{cn}$ & OMPB & $\begin{array}{c}\text { Division of } \\
\text { Industrial } \\
\text { Hygiene of the } \\
\text { Provincial Board } \\
\text { of Health }\end{array}$ & \\
\hline & & $\begin{array}{c}\text { Assosiated Screen News } \\
\text { LTD }\end{array}$ & & & & & & \\
\hline 273940 & $\begin{array}{c}\text { Assosiated } \\
\text { Screen News } \\
\text { LTD. } \\
\end{array}$ & $1973-0127$ & $\begin{array}{c}\text { Queen's } \\
\text { University } 1841- \\
1926: \text { Eighty Five } \\
\text { Years of Loyal } \\
\text { Service: } \\
\text { [incomplete] } \\
\end{array}$ & 1926 & $\mathrm{cn}$ & OMPB & & $\begin{array}{c}\text { Tinted } \\
\text { unknown } \\
\text { colour } \\
\end{array}$ \\
\hline & & $\begin{array}{c}\text { Hearsum, } \\
\text { Norman }\end{array}$ & & & & & & \\
\hline 335210 & $\begin{array}{c}\text { Hearsum, } \\
\text { Norman }\end{array}$ & 1973-0181 & Victory Loan 1919 & 1919 & $\mathrm{cn}$ & N/A & & $\begin{array}{c}\text { lack of } \\
\text { information }\end{array}$ \\
\hline 460894 & $\begin{array}{c}\text { Hearsum, } \\
\text { Norman }\end{array}$ & 1973-0181 & $\begin{array}{c}\text { Britain's Future } \\
\text { King } \\
\end{array}$ & 1919 & $\mathrm{cn}$ & $\begin{array}{l}\text { Pathescope of } \\
\text { Canada } \\
\end{array}$ & & \\
\hline & & \multicolumn{2}{|c|}{ National Library of Australia } & & & & & \\
\hline 23343 & $\begin{array}{l}\text { National } \\
\text { Library of } \\
\text { Australia }\end{array}$ & 1973-0182 & $\begin{array}{l}\text { Ontario's Summer } \\
\text { Sorceries } \\
\end{array}$ & 1924 & $\mathrm{cn}$ & OMPB & & \\
\hline & & \multicolumn{2}{|c|}{ Health Leauge of Canada } & & & & & \\
\hline 22976 & $\begin{array}{l}\text { Health Leauge } \\
\text { of Canada }\end{array}$ & 1973-0201 & $\begin{array}{l}\text { The Might of Pure } \\
\text { Milk }\end{array}$ & N/A & us & $\begin{array}{c}\text { National Motion } \\
\text { Picture } \\
\text { Company } \\
\end{array}$ & $\begin{array}{c}\text { The Canadian } \\
\text { Social Hygiene } \\
\text { Council }\end{array}$ & $\begin{array}{c}\text { National } \\
\text { motion } \\
\text { pictures } \\
\text { company } \\
\text { located in } \\
\text { Indianapolis } \\
\end{array}$ \\
\hline & & \multicolumn{2}{|c|}{$\begin{array}{c}\text { Provincial Archives of } \\
\text { Alberta } \\
\end{array}$} & & & & & \\
\hline 327489 & $\begin{array}{l}\text { Provincial } \\
\text { Archives of } \\
\text { Alberta } \\
\end{array}$ & 1973-0242 & $\begin{array}{l}\text { [Labour Saving in } \\
\text { the Home: } \\
\text { incomplete] }\end{array}$ & 1920/1929? & N/A & N/A & & \\
\hline 327535 & $\begin{array}{l}\text { Provincial } \\
\text { Archives of } \\
\text { Alberta } \\
\end{array}$ & $1973-0242$ & Oxygen & $1920 / 1925 ?$ & N/A & N/A & $\begin{array}{c}\text { University of } \\
\text { Alberta } \\
\text { Department of } \\
\text { Extension ; } \\
\text { Winged-foot } \\
\text { Travelling } \\
\text { Library of } \\
\text { Educational } \\
\text { Latern Slides } \\
\text { and Films } \\
\end{array}$ & \\
\hline 344441 & $\begin{array}{l}\text { Provincial } \\
\text { Archives of } \\
\text { Alberta } \\
\end{array}$ & $1973-0242$ & $\begin{array}{l}\text { The Great } \\
\text { American } \\
\text { Exercise }\end{array}$ & $\mathrm{N} / \mathrm{A}$ & us & $\begin{array}{l}\text { Paramount } \\
\text { Pictures } \\
\end{array}$ & & \\
\hline 379553 & $\begin{array}{l}\text { Provincial } \\
\text { Archives of } \\
\text { Alberta } \\
\end{array}$ & $1973-0242$ & $\begin{array}{l}\text { Liquid Air and } \\
\text { Application of } \\
\text { Intense Cold } \\
\end{array}$ & $1920 / 1925 ?$ & $\mathrm{~N} / \mathrm{A}$ & Pathe Freres & & \\
\hline 376676 & $\begin{array}{l}\text { Provincial } \\
\text { Archives of } \\
\text { Alberta } \\
\end{array}$ & $1973-0242$ & $\begin{array}{c}\text { Interesting } \\
\text { Experiments in } \\
\text { Physics } \\
\end{array}$ & 1916 & us & $\begin{array}{l}\text { Bray Studios } \\
\text { Inc. } \\
\end{array}$ & & $\begin{array}{c}\text { no } \\
\text { description } \\
\end{array}$ \\
\hline 415592 & $\begin{array}{l}\text { Provincial } \\
\text { Archives of } \\
\text { Alberta } \\
\end{array}$ & 1973-0242 & $\begin{array}{l}\text { The Rule of } \\
\text { Reason }\end{array}$ & 1920/1925? & us & N/A & & $\begin{array}{l}\text { producer/dir } \\
\text { ector, } \\
\text { Charles E. } \\
\text { Davenport; } \\
\text { photography } \\
\text {, Irving R. } \\
\text { Ruby } \\
\end{array}$ \\
\hline 415724 & $\begin{array}{l}\text { Provincial } \\
\text { Archives of } \\
\text { Alberta } \\
\end{array}$ & 1973-0242 & $\begin{array}{l}\text { Fur Farming in } \\
\text { Alberta }\end{array}$ & 1920/1929? & $\mathrm{cn}$ & $\begin{array}{c}\text { Alberta } \\
\text { Department of } \\
\text { Agriculture } \\
\text { Motion Pictures }\end{array}$ & & \\
\hline 417410 & $\begin{array}{l}\text { Provincial } \\
\text { Archives of } \\
\text { Alberta } \\
\end{array}$ & $1973-0242$ & $\begin{array}{l}\text { Physics Made } \\
\text { Amusing -- } \\
\text { Experiments With } \\
\text { Electricity } \\
\end{array}$ & 1920/1929? & us & Pathe Freres & & $\begin{array}{c}\text { Production } \\
\text { company } \\
\text { and country } \\
\text { of } \\
\text { production }\end{array}$ \\
\hline
\end{tabular}




\begin{tabular}{|c|c|c|c|c|c|c|c|c|c|c|}
\hline & & & & & & & & & & not sure \\
\hline 439156 & $\begin{array}{l}\text { Provincial } \\
\text { Archives of } \\
\text { Alberta } \\
\end{array}$ & 1973-0242 & & $\begin{array}{c}\text { Natural } \\
\text { Phenomena. Snow } \\
\text { and Ice } \\
\end{array}$ & 1920/1924? & N/A & Pathescope & & & \\
\hline 439157 & $\begin{array}{c}\text { Provincial } \\
\text { Archives of } \\
\text { Alberta } \\
\end{array}$ & $1973-0242$ & & $\begin{array}{c}\text { The Heart and } \\
\text { Circulation of the } \\
\text { Blood }\end{array}$ & 1920/1924? & N/A & Pathescope & & & \\
\hline 447497 & $\begin{array}{l}\text { Provincial } \\
\text { Archives of } \\
\text { Alberta } \\
\end{array}$ & 1973-0242 & & $\begin{array}{l}\text { The Tin Soldier } \\
\text { and the Dolls }\end{array}$ & 1914 & us & $\begin{array}{c}\text { Thanhouser Film } \\
\text { Corporation }\end{array}$ & & & \\
\hline 461047 & $\begin{array}{l}\text { Provincial } \\
\text { Archives of } \\
\text { Alberta } \\
\end{array}$ & $1973-0242$ & & $\begin{array}{l}\text { The Stream of } \\
\text { Life }\end{array}$ & 1919 & us & $\begin{array}{l}\text { Plimpton Epic } \\
\text { Films Inc; } \\
\text { Plymouth Film } \\
\text { Corporation } \\
\end{array}$ & & & $\begin{array}{c}\text { Tinted } \\
\text { unknown } \\
\text { colour }\end{array}$ \\
\hline & & \multicolumn{2}{|c|}{$\begin{array}{c}\text { Saskatchewan Archives } \\
\text { Board }\end{array}$} & & & & & & & \\
\hline 185842 & $\begin{array}{c}\text { Saskatchewan } \\
\text { Archives } \\
\text { Board } \\
\end{array}$ & $1974-0217$ & & $\begin{array}{c}\text { Farm Boy's } \\
\text { Camps }\end{array}$ & 1920 & $\mathrm{cn}$ & $\begin{array}{c}\text { Pathescope of } \\
\text { Canada } \\
\end{array}$ & & & \\
\hline 217098 & $\begin{array}{c}\text { Saskatchewan } \\
\text { Archives } \\
\text { Board } \\
\end{array}$ & $1974-0217$ & & [Life in a Beehive] & $1920 / 1929$ & $\mathrm{cn}$ & N/A & & & \\
\hline 217099 & $\begin{array}{c}\text { Saskatchewan } \\
\text { Archives } \\
\text { Board } \\
\end{array}$ & 1974-0217 & & Victory Loan 1918 & 1918 & $\mathrm{cn}$ & $\begin{array}{c}\text { Famous Players- } \\
\text { Lasky } \\
\end{array}$ & $\begin{array}{c}\text { Dominion } \\
\text { Publicity } \\
\text { Committee } \\
\end{array}$ & $\begin{array}{c}\text { Famous } \\
\text { Players Film } \\
\text { Services } \\
\end{array}$ & \\
\hline 217100 & $\begin{array}{c}\text { Saskatchewan } \\
\text { Archives } \\
\text { Board } \\
\end{array}$ & 1974-0217 & & $\begin{array}{c}\text { [A Visit to } \\
\text { Yosemite National } \\
\text { Park] } \\
\end{array}$ & 1910/1923 & us & N/A & & & $\begin{array}{l}\text { Tinted and } \\
\text { Toned } \\
\text { unknown } \\
\text { colours } \\
\end{array}$ \\
\hline 227899 & $\begin{array}{c}\text { Saskatchewan } \\
\text { Archives } \\
\text { Board } \\
\end{array}$ & 1974-0217 & & $\begin{array}{l}\text { Co-operative Live } \\
\text { Stock Marketing } \\
\text { in Saskatchewan } \\
\end{array}$ & 1923 & $\mathrm{cn}$ & N/A & & & \\
\hline 241272 & $\begin{array}{c}\text { Saskatchewan } \\
\text { Archives } \\
\text { Board } \\
\end{array}$ & 1974-0217 & & $\begin{array}{c}\text { [Regina Farm } \\
\text { Boys Camp] }= \\
\text { Farm Boy's } \\
\text { Camps } 1922 \\
\end{array}$ & $1922 ?$ & $\mathrm{cn}$ & $\begin{array}{c}\text { Pathescope of } \\
\text { Canada }\end{array}$ & & & $\begin{array}{c}\text { Possibly } \\
\text { filmed by } \\
\text { Dick Bird, } \\
\text { Regina } \\
\text { Films. } \\
\end{array}$ \\
\hline 283450 & $\begin{array}{c}\text { Saskatchewan } \\
\text { Archives } \\
\text { Board } \\
\end{array}$ & 1974-0217 & & $\begin{array}{c}\text { Bobby Bumps } \\
\text { Amusement Park }\end{array}$ & 1917 & us & $\begin{array}{c}\text { J.R. Bray } \\
\text { Studios Inc. }\end{array}$ & & $\begin{array}{c}\text { Paramount } \\
\text { Pictures }\end{array}$ & \\
\hline 335244 & $\begin{array}{c}\text { Saskatchewan } \\
\text { Archives } \\
\text { Board } \\
\end{array}$ & 1974-0217 & & $\begin{array}{l}\text { The Vicar of } \\
\text { Wakefield }\end{array}$ & 1917 & us & N/A & & & $\begin{array}{c}\text { Tinted } \\
\text { unknown } \\
\text { colour. } \\
\text { Little } \\
\text { decription } \\
\end{array}$ \\
\hline 360437 & $\begin{array}{c}\text { Saskatchewan } \\
\text { Archives } \\
\text { Board } \\
\end{array}$ & 1974-0217 & $\begin{array}{c}\text { Paramount- } \\
\text { Bray } \\
\text { Pictographs } \\
\end{array}$ & $\begin{array}{c}\text { Money Old and } \\
\text { New }\end{array}$ & 1917 & us & $\begin{array}{c}\text { Bray } \\
\text { Productions Inc. } \\
\end{array}$ & & & \\
\hline 364246 & $\begin{array}{c}\text { Saskatchewan } \\
\text { Archives } \\
\text { Board } \\
\end{array}$ & $1974-0217$ & & $\begin{array}{c}\text { A Crop of Frozen } \\
\text { Wheat }\end{array}$ & $1920 ?$ & $\mathrm{cn}$ & $\begin{array}{l}\text { Regina Films } \\
\text { Limited }\end{array}$ & $\begin{array}{l}\text { Department of } \\
\text { Agriculture, } \\
\text { Government of } \\
\text { Saskatchewan }\end{array}$ & & \\
\hline 369817 & $\begin{array}{c}\text { Saskatchewan } \\
\text { Archives } \\
\text { Board } \\
\end{array}$ & 1974-0217 & & $\begin{array}{c}\text { Co-operative } \\
\text { Marketing of Live } \\
\text { Stock } \\
\end{array}$ & 1920 & $\mathrm{cn}$ & $\begin{array}{c}\text { Pathescope of } \\
\text { Canada }\end{array}$ & & & \\
\hline 370739 & $\begin{array}{c}\text { Saskatchewan } \\
\text { Archives } \\
\text { Board } \\
\end{array}$ & 1974-0217 & & $\begin{array}{c}\text { Selecting a Dairy } \\
\text { Cow }\end{array}$ & 1920 & $\mathrm{cn}$ & $\begin{array}{c}\text { Pathescope of } \\
\text { Canada }\end{array}$ & & & \\
\hline 383458 & $\begin{array}{c}\text { Saskatchewan } \\
\text { Archives } \\
\text { Board } \\
\end{array}$ & 1974-0217 & $\begin{array}{l}\text { Out of the } \\
\text { Inkwell }\end{array}$ & Fishing & 1921 & us & $\begin{array}{l}\text { Out of the } \\
\text { Inkwell Films } \\
\text { Incorporated }\end{array}$ & & & \\
\hline 383459 & $\begin{array}{c}\text { Saskatchewan } \\
\text { Archives } \\
\text { Board } \\
\end{array}$ & 1974-0217 & $\begin{array}{c}\text { Out of the } \\
\text { Inkwell }\end{array}$ & $\begin{array}{c}\text { Jumping Beans : } \\
\text { [incomplete] }\end{array}$ & 1922 & us & $\begin{array}{l}\text { Out of the } \\
\text { Inkwell Films } \\
\text { Incorporated }\end{array}$ & & & $\begin{array}{r}\text { Tinted } \\
\text { yellow } \\
\end{array}$ \\
\hline 383460 & $\begin{array}{c}\text { Saskatchewan } \\
\text { Archives } \\
\text { Board } \\
\end{array}$ & $1974-0217$ & $\begin{array}{l}\text { Out of the } \\
\text { Inkwell }\end{array}$ & $\begin{array}{l}\text { The Challenge : } \\
\text { [incomplete] }\end{array}$ & 1922 & us & $\begin{array}{l}\text { Out of the } \\
\text { Inkwell Films } \\
\text { Incorporated }\end{array}$ & & & $\begin{array}{l}\text { Tinted } \\
\text { yellow }\end{array}$ \\
\hline
\end{tabular}




\begin{tabular}{|c|c|c|c|c|c|c|c|c|c|}
\hline 383461 & $\begin{array}{c}\text { Saskatchewan } \\
\text { Archives } \\
\text { Board } \\
\end{array}$ & $1974-0217$ & & $\begin{array}{c}\text { The Little White } \\
\text { Girl }\end{array}$ & 1917 & us & $\begin{array}{l}\text { Essanay Film } \\
\text { Manufacturing } \\
\text { Company }\end{array}$ & & $\begin{array}{c}\text { Tinted } \\
\text { yellow and } \\
\text { red }\end{array}$ \\
\hline 383462 & $\begin{array}{c}\text { Saskatchewan } \\
\text { Archives } \\
\text { Board } \\
\end{array}$ & $1974-0217$ & & $\begin{array}{c}\text { A Dog's Love = } \\
\text { On Account of a } \\
\text { Dog }\end{array}$ & $1914 ?$ & us & $\begin{array}{c}\text { Thanhouser Film } \\
\text { Corporation }\end{array}$ & & \\
\hline 383463 & $\begin{array}{c}\text { Saskatchewan } \\
\text { Archives } \\
\text { Board } \\
\end{array}$ & $1974-0217$ & & $\begin{array}{l}\text { One Girl in a } \\
\text { Thousand }\end{array}$ & N/A & us & $\begin{array}{c}\text { Essanay Film } \\
\text { Manufacturing } \\
\text { Company }\end{array}$ & & \\
\hline 383464 & $\begin{array}{c}\text { Saskatchewan } \\
\text { Archives } \\
\text { Board } \\
\end{array}$ & $1974-0217$ & & $\begin{array}{l}\text { Chestnuts : } \\
\text { [incomplete] }\end{array}$ & 1919/1924? & us & $\begin{array}{c}\text { Pat Sullivan } \\
\text { Studio } \\
\end{array}$ & & $\begin{array}{l}\text { Tinted } \\
\text { yellow }\end{array}$ \\
\hline 383465 & $\begin{array}{c}\text { Saskatchewan } \\
\text { Archives } \\
\text { Board } \\
\end{array}$ & $1974-0217$ & & $\begin{array}{l}\text { Cricket on the } \\
\text { Hearth : } \\
\text { [incomplete] }\end{array}$ & 1914 & us & $\begin{array}{l}\text { Biograph } \\
\text { Company }\end{array}$ & $\begin{array}{l}\text { University of } \\
\text { Alberta } \\
\text { Department of } \\
\text { Extension } \\
\text { Wing Foot } \\
\text { Travelling } \\
\text { Library of } \\
\text { Educational } \\
\text { Lantern Slides } \\
\text { and Films. } \\
\end{array}$ & \\
\hline 383466 & $\begin{array}{c}\text { Saskatchewan } \\
\text { Archives } \\
\text { Board } \\
\end{array}$ & $1974-0217$ & & $\begin{array}{c}\text { The Magic Toy } \\
\text { Shop }\end{array}$ & $1915 / 1925 ?$ & N/A & Pathe Freres & & \\
\hline 383467 & $\begin{array}{c}\text { Saskatchewan } \\
\text { Archives } \\
\text { Board } \\
\end{array}$ & $1974-0217$ & & Easter Eggs & 1920/1924? & $\mathrm{fr}$ & N/A & & \\
\hline 383468 & $\begin{array}{c}\text { Saskatchewan } \\
\text { Archives } \\
\text { Board } \\
\end{array}$ & $1974-0217$ & & $\begin{array}{c}\text { Manufacture of } \\
\text { Bamboo Hats }\end{array}$ & $1920 / 1922$ & fr & Pathe Freres & & $\begin{array}{l}\text { Tinted } \\
\text { yellow }\end{array}$ \\
\hline 383469 & $\begin{array}{c}\text { Saskatchewan } \\
\text { Archives } \\
\text { Board } \\
\end{array}$ & $1974-0217$ & $\begin{array}{c}\text { From Seed } \\
\text { to Wearer }\end{array}$ & $\begin{array}{c}\text { Manufacture of } \\
\text { Fine Cotton Cloth }\end{array}$ & 1917/1924? & us & $\begin{array}{c}\text { Amoskeag } \\
\text { Manufacturing } \\
\text { Company }\end{array}$ & $\begin{array}{l}\text { University of } \\
\text { Alberta } \\
\text { Department of } \\
\text { Extension } \\
\text { Winged-foot } \\
\text { Tavelling } \\
\text { Library of } \\
\text { Educational } \\
\text { Lantern Slides } \\
\text { and Films } \\
\end{array}$ & \\
\hline 383471 & $\begin{array}{c}\text { Saskatchewan } \\
\text { Archives } \\
\text { Board } \\
\end{array}$ & 1974-0217 & & Silas Marner & 1916 & us & $\begin{array}{c}\text { Thanhouser Film } \\
\text { Corporation }\end{array}$ & $\begin{array}{l}\text { Mutual Film } \\
\text { Corporation }\end{array}$ & \\
\hline 383472 & $\begin{array}{c}\text { Saskatchewan } \\
\text { Archives } \\
\text { Board } \\
\end{array}$ & $1974-0217$ & & Cinderella & 1911 & us & $\begin{array}{c}\text { Selig-Polyscope } \\
\text { Company }\end{array}$ & & \\
\hline 383474 & $\begin{array}{c}\text { Saskatchewan } \\
\text { Archives } \\
\text { Board } \\
\end{array}$ & $1974-0217$ & & Old Homeless & N/A & N/A & N/A & $\begin{array}{l}\text { Univeristy of } \\
\text { Alberta } \\
\text { Department of } \\
\text { Extension } \\
\text { Winged-foot } \\
\text { Travelling } \\
\text { Library of } \\
\text { Educational } \\
\text { Lantern Slides } \\
\text { and Films } \\
\end{array}$ & \\
\hline 383475 & $\begin{array}{c}\text { Saskatchewan } \\
\text { Archives } \\
\text { Board } \\
\end{array}$ & $1974-0217$ & & $\begin{array}{c}\text { Shorthorns at } \\
\text { Chicago }\end{array}$ & 1920/1923? & $\mathrm{N} / \mathrm{A}$ & N/A & & \\
\hline 383476 & $\begin{array}{c}\text { Saskatchewan } \\
\text { Archives } \\
\text { Board } \\
\end{array}$ & $1974-0217$ & & Sunshine and Gold & $1917 ?$ & us & Balboa-Pathe & $\begin{array}{l}\text { Univeristy of } \\
\text { Alberta } \\
\text { Department of } \\
\text { Extension } \\
\text { Winged-foot } \\
\text { Travelling } \\
\text { Library of } \\
\text { Educational } \\
\text { Lantern } \\
\end{array}$ & \\
\hline
\end{tabular}




\begin{tabular}{|c|c|c|c|c|c|c|c|c|c|c|}
\hline 383477 & $\begin{array}{c}\text { Saskatchewan } \\
\text { Archives } \\
\text { Board } \\
\end{array}$ & $1974-0217$ & & $\begin{array}{c}\text { Twinkle Twinkle } \\
\text { Little Star }\end{array}$ & $1918 / 1923 ?$ & N/A & $\mathrm{N} / \mathrm{A}$ & & $\begin{array}{c}\text { Univeristy of } \\
\text { Alberta } \\
\text { Department of } \\
\text { Extension } \\
\text { Winged-foot } \\
\text { Travelling } \\
\text { Library of } \\
\text { Educational } \\
\text { Lantern } \\
\end{array}$ & $\begin{array}{l}\text { Tinted } \\
\text { unknown } \\
\text { Colour } \\
\end{array}$ \\
\hline 383478 & $\begin{array}{c}\text { Saskatchewan } \\
\text { Archives } \\
\text { Board } \\
\end{array}$ & $1974-0217$ & $\begin{array}{c}\text { Pathé } \\
\text { Review }\end{array}$ & [Cinematograph] & $1920 / 1930 ?$ & us & $\begin{array}{l}\text { Novagraph Film } \\
\text { Corporation; } \\
\text { Pathescope }\end{array}$ & & & \\
\hline 383480 & $\begin{array}{c}\text { Saskatchewan } \\
\text { Archives } \\
\text { Board } \\
\end{array}$ & $1974-0217$ & & $\begin{array}{l}\text { Livestock on the } \\
\text { University Farm }= \\
\text { [Livestock on the } \\
\text { University of } \\
\text { Saskatchewan } \\
\text { Farm] }\end{array}$ & $1926 / 1927 ?$ & $\mathrm{cn}$ & $\begin{array}{l}\text { Regina Films } \\
\text { Limited }\end{array}$ & & & \\
\hline 383481 & $\begin{array}{c}\text { Saskatchewan } \\
\text { Archives } \\
\text { Board } \\
\end{array}$ & 1974-0217 & & The Silken Web & 1920/1924? & $\mathrm{cn}$ & $\mathrm{N} / \mathrm{A}$ & & $\begin{array}{c}\text { University of } \\
\text { Alberta } \\
\text { Department of } \\
\text { Extension } \\
\text { Winged-foot } \\
\text { Travelling } \\
\text { Library of } \\
\text { Educational } \\
\text { Lantern } \\
\end{array}$ & $\begin{array}{c}\text { Tinted } \\
\text { variety of } \\
\text { colours }\end{array}$ \\
\hline 415725 & $\begin{array}{c}\text { Saskatchewan } \\
\text { Archives } \\
\text { Board } \\
\end{array}$ & 1974-0217 & & $\begin{array}{c}\text { [Herd Control -- } \\
\text { Testing For Milk } \\
\text { Diseases] }\end{array}$ & 1919 & $\mathrm{cn}$ & $\mathrm{N} / \mathrm{A}$ & & & \\
\hline 415726 & $\begin{array}{c}\text { Saskatchewan } \\
\text { Archives } \\
\text { Board } \\
\end{array}$ & $1974-0217$ & & $\begin{array}{c}\text { [Poultry in } \\
\text { Saskatchewan] : } \\
\text { excerpt }\end{array}$ & 1922 & $\mathrm{cn}$ & $\mathrm{N} / \mathrm{A}$ & & & $\begin{array}{c}\text { Tinted } \\
\text { unknown } \\
\text { colour }\end{array}$ \\
\hline 415728 & $\begin{array}{c}\text { Saskatchewan } \\
\text { Archives } \\
\text { Board } \\
\end{array}$ & 1974-0217 & & [Sifting Wheat] & 1920 & $\mathrm{cn}$ & $\mathrm{N} / \mathrm{A}$ & & & \\
\hline 415732 & $\begin{array}{c}\text { Saskatchewan } \\
\text { Archives } \\
\text { Board } \\
\end{array}$ & 1974-0217 & & $\begin{array}{l}\text { [Cultivating the } \\
\text { Prairies With } \\
\text { Horses] }\end{array}$ & 1920/1929? & $\mathrm{cn}$ & $\mathrm{N} / \mathrm{A}$ & & & \\
\hline 415733 & $\begin{array}{c}\text { Saskatchewan } \\
\text { Archives } \\
\text { Board } \\
\end{array}$ & 1974-0217 & & $\begin{array}{c}\text { [Stock Threshing } \\
\text { Near the City of } \\
\text { Regina] }\end{array}$ & 1924 & $\mathrm{cn}$ & $\mathrm{N} / \mathrm{A}$ & & & \\
\hline 416398 & $\begin{array}{c}\text { Saskatchewan } \\
\text { Archives } \\
\text { Board } \\
\end{array}$ & 1974-0217 & & $\begin{array}{c}\text { Tuberculosis in } \\
\text { Saskatchewan }\end{array}$ & 1920 & $\mathrm{cn}$ & $\mathrm{N} / \mathrm{A}$ & & & \\
\hline 440614 & $\begin{array}{c}\text { Saskatchewan } \\
\text { Archives } \\
\text { Board } \\
\end{array}$ & $1974-0217$ & & Grading Stallions & 1920 & $\mathrm{cn}$ & $\begin{array}{c}\text { Pathescope of } \\
\text { Canada }\end{array}$ & $\begin{array}{c}\text { Saskatchewan } \\
\text { Government } \\
\text { Bureau of } \\
\text { Publications, } \\
\text { Motion Picture } \\
\text { Branch } \\
\end{array}$ & & \\
\hline 440745 & $\begin{array}{c}\text { Saskatchewan } \\
\text { Archives } \\
\text { Board } \\
\end{array}$ & 1974-0217 & & $\begin{array}{l}\text { Cream Grading in } \\
\text { Saskatchewan : } \\
\text { incomplete }\end{array}$ & $\mathrm{N} / \mathrm{A}$ & $\mathrm{cn}$ & $\begin{array}{c}\text { Regina Films } \\
\text { Limited }\end{array}$ & $\begin{array}{c}\text { Saskatchewan } \\
\text { Government }\end{array}$ & & $\begin{array}{c}\text { Tinted and } \\
\text { toned }\end{array}$ \\
\hline 444664 & $\begin{array}{c}\text { Saskatchewan } \\
\text { Archives } \\
\text { Board } \\
\end{array}$ & $1974-0217$ & & $\begin{array}{c}\text { Saskatchewan's } \\
\text { War On the } \\
\text { Grasshopper }\end{array}$ & 1920 & $\mathrm{cn}$ & $\begin{array}{l}\text { Pathescope of } \\
\text { Canada }\end{array}$ & $\begin{array}{c}\text { Saskatchewan } \\
\text { Department of } \\
\text { Agriculture }\end{array}$ & & $\begin{array}{c}\text { tinted } \\
\text { unknown } \\
\text { colours }\end{array}$ \\
\hline 458639 & $\begin{array}{c}\text { Saskatchewan } \\
\text { Archives } \\
\text { Board } \\
\end{array}$ & $1974-0217$ & & $\begin{array}{c}\text { [Egg and Poultry } \\
\text { Farming in } \\
\text { Saskatchewan] : } \\
\text { [incomplete] }\end{array}$ & 1920/1929? & $\mathrm{cn}$ & $\mathrm{N} / \mathrm{A}$ & & & \\
\hline 19698 & $\begin{array}{c}\text { Saskatchewan } \\
\text { Archives } \\
\text { Board } \\
\end{array}$ & $1974-0217$ & & $\begin{array}{c}\text { Queen's } \\
\text { University } 1841- \\
1926: \text { Eighty Five } \\
\text { Years of Loyal } \\
\text { Service: } \\
\text { [incomplete] }\end{array}$ & N/A & $\mathrm{cn}$ & $\mathrm{N} / \mathrm{A}$ & & & $\begin{array}{l}\text { Tinted } \\
\text { yellow }\end{array}$ \\
\hline 22599 & $\begin{array}{c}\text { Saskatchewan } \\
\text { Archives } \\
\text { Board } \\
\end{array}$ & $1974-0217$ & & $\begin{array}{c}\text { How to Live Long } \\
\text { and Well }\end{array}$ & 1926 & us & $\begin{array}{c}\text { National Motion } \\
\text { Pictures } \\
\text { Compnay } \\
\end{array}$ & $\begin{array}{l}\text { Canadian Social } \\
\text { Hygiene Council }\end{array}$ & & \\
\hline
\end{tabular}




\begin{tabular}{|c|c|c|c|c|c|c|c|c|c|}
\hline 24019 & $\begin{array}{c}\text { Saskatchewan } \\
\text { Archives } \\
\text { Board }\end{array}$ & $1974-0217$ & $\begin{array}{l}\text { Cream Grading in } \\
\text { Saskatchewan }\end{array}$ & 1925 & $\mathrm{cn}$ & $\begin{array}{l}\text { Regina Films } \\
\text { Limited }\end{array}$ & $\begin{array}{c}\text { Saskatchewan } \\
\text { Government }\end{array}$ & & $\begin{array}{l}\text { Tinted } \\
\text { unknow } \\
\text { colour. } \\
\text { Printed on } \\
\text { Eastman } \\
\text { stock not } \\
\text { Pathe }\end{array}$ \\
\hline 28244 & $\begin{array}{c}\text { Saskatchewan } \\
\text { Archives } \\
\text { Board } \\
\end{array}$ & 1974-0217 & $\begin{array}{c}\text { Butter Making in } \\
\text { New Ontario = } \\
\text { Butter Making in } \\
\text { Ontario } \\
\end{array}$ & $1918 / 1927$ & $\mathrm{cn}$ & OMPB & & & \\
\hline 28245 & $\begin{array}{c}\text { Saskatchewan } \\
\text { Archives } \\
\text { Board } \\
\end{array}$ & $1974-0217$ & $\begin{array}{c}\text { Saskatchewan's } \\
\text { War On the } \\
\text { Grasshopper }\end{array}$ & 1920 & $\mathrm{cn}$ & $\begin{array}{c}\text { Pathescope of } \\
\text { Canada }\end{array}$ & $\begin{array}{c}\text { Saskatchewan } \\
\text { Department of } \\
\text { Agriculture }\end{array}$ & & \\
\hline 28246 & $\begin{array}{c}\text { Saskatchewan } \\
\text { Archives } \\
\text { Board } \\
\end{array}$ & 1974-0217 & $\begin{array}{c}\text { Cream Grading - } \\
\text { Why? }\end{array}$ & 1921 & $\mathrm{cn}$ & $\begin{array}{c}\text { Pathescope of } \\
\text { Canada } \\
\end{array}$ & $\begin{array}{c}\text { Government of } \\
\text { Saskatchewan }\end{array}$ & & \\
\hline 28248 & $\begin{array}{c}\text { Saskatchewan } \\
\text { Archives } \\
\text { Board } \\
\end{array}$ & 1974-0217 & $\begin{array}{c}\text { What's in a Name? } \\
: \text { : incomplete] }\end{array}$ & 1919 & $\mathrm{cn}$ & $\begin{array}{c}\text { Pathescope of } \\
\text { Canada }\end{array}$ & & OMPB & \\
\hline 28249 & $\begin{array}{c}\text { Saskatchewan } \\
\text { Archives } \\
\text { Board } \\
\end{array}$ & $1974-0217$ & $\begin{array}{c}\text { University of } \\
\text { Saskatchewan } \\
\text { Projects } \\
\end{array}$ & $1925 ?$ & $\mathrm{cn}$ & N/A & & & \\
\hline 28250 & $\begin{array}{c}\text { Saskatchewan } \\
\text { Archives } \\
\text { Board }\end{array}$ & $1974-0217$ & $\begin{array}{c}\text { [Farming in } \\
\text { Saskatchewan : } \\
\text { outtakes }= \\
\text { Saskatchewan } \\
\text { Farming] }\end{array}$ & 1921 & $\mathrm{cn}$ & $\begin{array}{l}\text { Regina Films } \\
\text { Limited }\end{array}$ & & & $\begin{array}{c}\text { Tinted } \\
\text { yellow. } \\
\text { Outtakes } \\
\text { make it } \\
\text { unusual }\end{array}$ \\
\hline 28251 & $\begin{array}{c}\text { Saskatchewan } \\
\text { Archives } \\
\text { Board } \\
\end{array}$ & 1974-0217 & $\begin{array}{l}\text { Culling Hens : } \\
\text { [incomplete] }\end{array}$ & 1923 & $\mathrm{cn}$ & $\begin{array}{c}\text { Filmcraft } \\
\text { Industries Ltd. }\end{array}$ & OMPB & & \\
\hline 28252 & $\begin{array}{c}\text { Saskatchewan } \\
\text { Archives } \\
\text { Board } \\
\end{array}$ & $1974-0217$ & $\begin{array}{c}\text { Co-op Livestock } \\
\text { Marketing = } \\
\text { United Grain } \\
\text { Growers Co- } \\
\text { operative } \\
\text { Livestock } \\
\text { Marketing = Co- } \\
\text { operative } \\
\text { Marketing of } \\
\text { Livestock } \\
\end{array}$ & 1920 & $\mathrm{cn}$ & $\begin{array}{l}\text { Pathescope of } \\
\text { Canada }\end{array}$ & & & \\
\hline 28253 & $\begin{array}{c}\text { Saskatchewan } \\
\text { Archives } \\
\text { Board } \\
\end{array}$ & 1974-0217 & $\begin{array}{c}\text { [Better Bulls : } \\
\text { incomplete] }\end{array}$ & 1924 & $\mathrm{cn}$ & $\begin{array}{c}\text { Saskatchewan } \\
\text { Government } \\
\text { Bureau of } \\
\text { Publications, } \\
\text { Motion Picture } \\
\text { Branch } \\
\end{array}$ & & & \\
\hline 28254 & $\begin{array}{c}\text { Saskatchewan } \\
\text { Archives } \\
\text { Board } \\
\end{array}$ & $1974-0217$ & $\begin{array}{l}\text { Hog Raising in } \\
\text { Saskatchewan : } \\
\text { [incomplete] }\end{array}$ & 1920 & $\mathrm{cn}$ & $\mathrm{N} / \mathrm{A}$ & & & \\
\hline 28255 & $\begin{array}{c}\text { Saskatchewan } \\
\text { Archives } \\
\text { Board } \\
\end{array}$ & $1974-0217$ & $\begin{array}{c}\text { Poultry Marketing } \\
\text { in Saskatchewan }\end{array}$ & $1920 / 1929$ & $\mathrm{cn}$ & N/A & & & \\
\hline 28256 & $\begin{array}{c}\text { Saskatchewan } \\
\text { Archives } \\
\text { Board } \\
\end{array}$ & 1974-0217 & $\begin{array}{c}\text { Embryology of the } \\
\text { Egg }\end{array}$ & 1920 & $\mathrm{cn}$ & N/A & & & \\
\hline 28257 & $\begin{array}{c}\text { Saskatchewan } \\
\text { Archives } \\
\text { Board } \\
\end{array}$ & $1974-0217$ & $\begin{array}{c}\text { Co-operative } \\
\text { Marketing of } \\
\text { Pedigreed Seed in } \\
\text { Saskatchewan }\end{array}$ & $1925 / 1929$ & $\mathrm{cn}$ & $\begin{array}{l}\text { Regina Films } \\
\text { Limited }\end{array}$ & & & \\
\hline 28258 & $\begin{array}{c}\text { Saskatchewan } \\
\text { Archives } \\
\text { Board } \\
\end{array}$ & $1974-0217$ & $\begin{array}{c}\text { Growing Trees } \\
\text { Plants on the } \\
\text { Prairies } \\
\end{array}$ & $1920 / 1929$ & $\mathrm{cn}$ & N/A & & & $\begin{array}{c}\text { Notes } \\
\text { suggests } \\
\text { department } \\
\text { of } \\
\text { agriculture } \\
\text { took part in } \\
\text { production? }\end{array}$ \\
\hline 28259 & $\begin{array}{c}\text { Saskatchewan } \\
\text { Archives } \\
\text { Board }\end{array}$ & $1974-0217$ & $\begin{array}{l}\text { Tapping Niagara's } \\
\text { Power Resources: } \\
\text { [incomplete] }\end{array}$ & 1922 & $\mathrm{cn}$ & $\begin{array}{c}\text { Filmcraft } \\
\text { Industries Ltd. }\end{array}$ & OMPB & & \\
\hline
\end{tabular}




\begin{tabular}{|c|c|c|c|c|c|c|c|c|c|c|}
\hline 28260 & $\begin{array}{l}\text { Saskatchewan } \\
\text { Archives } \\
\text { Board } \\
\end{array}$ & $1974-0217$ & & $\begin{array}{l}\text { Bobby Bumps } \\
\text { Outwits the } \\
\text { Dogcatcher }\end{array}$ & 1917 & us & $\begin{array}{c}\text { Bray } \\
\text { Productions Inc. }\end{array}$ & & $\begin{array}{l}\text { Paramount } \\
\text { Pictures }\end{array}$ & $\begin{array}{l}\text { Tinted } \\
\text { orange }\end{array}$ \\
\hline 28261 & $\begin{array}{c}\text { Saskatchewan } \\
\text { Archives } \\
\text { Board }\end{array}$ & $1974-0217$ & & Grading Stallions & $1920 / 1929$ & $\mathrm{cn}$ & $\begin{array}{c}\text { Saskatchewan } \\
\text { Government } \\
\text { Bureau of } \\
\text { Publications, } \\
\text { Motion Picture } \\
\text { Branch }\end{array}$ & & & \\
\hline 28262 & $\begin{array}{c}\text { Saskatchewan } \\
\text { Archives } \\
\text { Board } \\
\end{array}$ & 1974-0217 & & $\begin{array}{c}\text { Proportioning } \\
\text { Concrete } \\
\text { Mixtures. Mixing } \\
\text { and Placing } \\
\text { Concrete } \\
\end{array}$ & 1920/1921? & $\mathrm{cn}$ & $\begin{array}{c}\text { Pathescope of } \\
\text { Canada }\end{array}$ & $\begin{array}{c}\text { OMPB; Canada } \\
\text { Cement } \\
\text { Company } \\
\end{array}$ & & \\
\hline 28263 & $\begin{array}{c}\text { Saskatchewan } \\
\text { Archives } \\
\text { Board } \\
\end{array}$ & $1974-0217$ & & $\begin{array}{c}\text { Where East Meets } \\
\text { West } \\
\end{array}$ & $1921 / 1925 ?$ & $\mathrm{cn}$ & OMPB & & & \\
\hline 28264 & $\begin{array}{c}\text { Saskatchewan } \\
\text { Archives } \\
\text { Board } \\
\end{array}$ & $1974-0217$ & $\begin{array}{l}\text { Canadian } \\
\text { National } \\
\text { Pictorial } \\
\end{array}$ & $\begin{array}{l}\text { The Annual } \\
\text { Varsity Scrap }\end{array}$ & 1919 & $\mathrm{cn}$ & $\begin{array}{l}\text { Pathescope of } \\
\text { Canada }\end{array}$ & & & $\begin{array}{l}\text { Tinted } \\
\text { yellow }\end{array}$ \\
\hline 28265 & $\begin{array}{c}\text { Saskatchewan } \\
\text { Archives } \\
\text { Board } \\
\end{array}$ & $1974-0217$ & & $\begin{array}{l}\text { Selecting a Dairy } \\
\text { Cow : incomplete }\end{array}$ & $1920 / 1929$ & $\mathrm{cn}$ & $\begin{array}{c}\text { Pathescope of } \\
\text { Canada }\end{array}$ & & & $\begin{array}{c}\text { production } \\
\text { demonstrate } \\
\mathrm{d} \text { by } \\
\text { Professor A. } \\
\text { M. Shaw of } \\
\text { the } \\
\text { University } \\
\text { of } \\
\text { Saskatchewa } \\
\text { n. }\end{array}$ \\
\hline 28266 & $\begin{array}{l}\text { Saskatchewan } \\
\text { Archives } \\
\text { Board } \\
\end{array}$ & $1974-0217$ & & $\begin{array}{l}\text { [Growing } \\
\text { Sunflowers] }\end{array}$ & $1920 / 1929$ & $\mathrm{cn}$ & $\mathrm{N} / \mathrm{A}$ & & & \\
\hline 28267 & $\begin{array}{c}\text { Saskatchewan } \\
\text { Archives } \\
\text { Board } \\
\end{array}$ & $1974-0217$ & & $\begin{array}{l}\text { Boys and Girls } \\
\text { Stock Judging } \\
\text { Competition }\end{array}$ & $1920 / 1929$ & $\mathrm{cn}$ & N/A & & & \\
\hline 28268 & $\begin{array}{c}\text { Saskatchewan } \\
\text { Archives } \\
\text { Board } \\
\end{array}$ & $1974-0217$ & & $\begin{array}{l}\text { Clearing New } \\
\text { Prairie Land }\end{array}$ & 1919 & $\mathrm{cn}$ & N/A & & & \\
\hline 28269 & $\begin{array}{l}\text { Saskatchewan } \\
\text { Archives } \\
\text { Board } \\
\end{array}$ & $1974-0217$ & & $\begin{array}{l}\text { [Dairy Farming } \\
\text { Industry in } \\
\text { Saskatchewan] }\end{array}$ & $1920 / 1929$ & $\mathrm{cn}$ & N/A & & & \\
\hline 28270 & $\begin{array}{c}\text { Saskatchewan } \\
\text { Archives } \\
\text { Board } \\
\end{array}$ & $1974-0217$ & & $\begin{array}{l}\text { Nation Building in } \\
\text { Saskatchewan }\end{array}$ & 1921 & $\mathrm{cn}$ & $\mathrm{N} / \mathrm{A}$ & & & \\
\hline 28271 & $\begin{array}{l}\text { Saskatchewan } \\
\text { Archives } \\
\text { Board } \\
\end{array}$ & $1974-0217$ & & $\begin{array}{l}\text { Preparing Poultry } \\
\text { For Market }\end{array}$ & $1920 / 1929$ & $\mathrm{cn}$ & N/A & & & \\
\hline 28272 & $\begin{array}{c}\text { Saskatchewan } \\
\text { Archives } \\
\text { Board } \\
\end{array}$ & $1974-0217$ & & $\begin{array}{c}\text { Growing } \\
\text { Registered Seed in } \\
\text { Saskatchewan }\end{array}$ & N/A & $\mathrm{cn}$ & N/A & & & \\
\hline & & $\begin{array}{c}\text { Canadian } \\
\text { Film Institue }\end{array}$ & & & & & & & & \\
\hline 440610 & $\begin{array}{l}\text { Canadian Film } \\
\text { Institue }\end{array}$ & $1975-0206$ & & The Hick & 1917 & us & Ince-Triangle & & $\begin{array}{l}\text { United Picture } \\
\text { Exchage }\end{array}$ & $\begin{array}{l}\text { Tinting at } \\
\text { the end }\end{array}$ \\
\hline \multirow[t]{2}{*}{467736} & $\begin{array}{c}\text { Canadian Film } \\
\text { Institue }\end{array}$ & $1975-0206$ & & Victory Loan & 1918 & $\mathrm{cn}$ & $\begin{array}{c}\text { Famous Players- } \\
\text { Lasky }\end{array}$ & $\begin{array}{c}\text { Dominion } \\
\text { Publicity } \\
\text { Committee } \\
\end{array}$ & $\begin{array}{c}\text { Famous } \\
\text { Players Film } \\
\text { Services } \\
\end{array}$ & \\
\hline & & Bell Canada & & & & & & & & \\
\hline 194551 & Bell Canada & $1975-0210$ & & $\begin{array}{l}\text { The Spirit of } \\
\text { Service }\end{array}$ & $1930 / 1939$ & $\mathrm{cn}$ & N/A & & & $\begin{array}{c}\text { Tinted } \\
\text { unknown } \\
\text { colour. } \\
\text { Film, acted } \\
\text { by telephone } \\
\text { employees } \\
\text { in the } \\
\text { Toronto } \\
\text { Division of } \\
\text { the Bell } \\
\text { Telephone }\end{array}$ \\
\hline
\end{tabular}




\begin{tabular}{|c|c|c|c|c|c|c|c|c|c|c|}
\hline & & & & & & & & & & Company. \\
\hline 324983 & Bell Canada & $1975-0210$ & & $\begin{array}{c}\text { The Telephone } \\
\text { Girl : [incomplete] }\end{array}$ & N/A & $\mathrm{cn}$ & $\begin{array}{l}\text { Pathescope of } \\
\text { Canada }\end{array}$ & & & \\
\hline 443604 & Bell Canada & 1975-0210 & & $\begin{array}{l}\text { Mr. Ruff Learns } \\
\text { His Lesson }\end{array}$ & 1920/1930? & $\mathrm{cn}$ & N/A & $\begin{array}{c}\text { The Bell } \\
\text { Telephone } \\
\text { Company of } \\
\text { Canada } \\
\end{array}$ & & $\begin{array}{c}\text { Tinted } \\
\text { unknown } \\
\text { colour } \\
\end{array}$ \\
\hline 443606 & Bell Canada & $1975-0210$ & & $\begin{array}{c}\text { Teaming up for } \\
\text { First Aid }\end{array}$ & $1921 ?$ & us & $\mathrm{N} / \mathrm{A}$ & $\begin{array}{c}\text { American } \\
\text { Telephone and } \\
\text { Telegraph } \\
\text { Company } \\
\end{array}$ & & $\begin{array}{c}\text { Tinted } \\
\text { unknown } \\
\text { colour }\end{array}$ \\
\hline 444039 & Bell Canada & 1975-0210 & & $\begin{array}{c}\text { The Telephone } \\
\text { Girl }\end{array}$ & N/A & $\mathrm{cn}$ & $\begin{array}{c}\text { Pathescope of } \\
\text { Canada }\end{array}$ & & & \\
\hline & & $\begin{array}{c}\text { Moravian } \\
\text { Church }\end{array}$ & & & & & & & & \\
\hline 111115 & $\begin{array}{l}\text { Moravian } \\
\text { Church }\end{array}$ & $1977-0231$ & $\begin{array}{l}\text { Paramount } \\
\text { Pictographs }\end{array}$ & $\begin{array}{c}\text { The Man Behind : } \\
\text { How a Motion } \\
\text { Picture is } \\
\text { Projected - The } \\
\text { Man - The } \\
\text { Machine }\end{array}$ & N/A & us & Paramount & & OMPB & \\
\hline 185112 & $\begin{array}{l}\text { Moravian } \\
\text { Church }\end{array}$ & 1977-0231 & $\begin{array}{l}\text { Canadian } \\
\text { National } \\
\text { Pictorial } \\
\end{array}$ & $\begin{array}{c}\text { Toronto Ontario : } \\
\text { Dedication of } \\
\text { Colours at Upper } \\
\text { Canada College } \\
\end{array}$ & 1919/1921? & $\mathrm{cn}$ & $\begin{array}{l}\text { Pathescope of } \\
\text { Canada }\end{array}$ & & OMPB & \\
\hline 232546 & $\begin{array}{c}\text { Moravian } \\
\text { Church }\end{array}$ & $1977-0231$ & & The Electric Bell & 1918 & us & Daypho-Bray & & & $\begin{array}{c}\text { Tinted } \\
\text { unknown } \\
\text { colour } \\
\end{array}$ \\
\hline 234375 & $\begin{array}{l}\text { Moravian } \\
\text { Church }\end{array}$ & $1977-0231$ & $\begin{array}{l}\text { Seeing } \\
\text { Canada }\end{array}$ & A City of Homes & 1924/1926? & $\mathrm{cn}$ & CGMPB & & & $\begin{array}{c}\text { Tinted } \\
\text { unknown } \\
\text { colour } \\
\end{array}$ \\
\hline 234376 & $\begin{array}{l}\text { Moravian } \\
\text { Church }\end{array}$ & $1977-0231$ & & $\begin{array}{c}\text { Harvesters of the } \\
\text { Sea = Harvesting } \\
\text { the Sea }\end{array}$ & 1918/1921? & $\mathrm{cn}$ & $\begin{array}{l}\text { Pathescope of } \\
\text { Canada }\end{array}$ & & & \\
\hline 234377 & $\begin{array}{l}\text { Moravian } \\
\text { Church }\end{array}$ & $1977-0231$ & & $\begin{array}{c}\text { Lake of Bays } \\
\text { Regatta }\end{array}$ & 1918/1922? & $\mathrm{cn}$ & $\begin{array}{c}\text { Pathescope of } \\
\text { Canada }\end{array}$ & & & $\begin{array}{c}\text { Lake of } \\
\text { Bays } \\
\text { Regatta in } \\
\text { Muskoka, } \\
\text { Ontario. } \\
\end{array}$ \\
\hline 234378 & $\begin{array}{l}\text { Moravian } \\
\text { Church }\end{array}$ & $1977-0231$ & & $\begin{array}{c}\text { Fair Days in } \\
\text { Picton }\end{array}$ & 1919 & $\mathrm{cn}$ & $\begin{array}{c}\text { Ontario Motion } \\
\text { Picture Bureau }\end{array}$ & & & \\
\hline 234379 & $\begin{array}{l}\text { Moravian } \\
\text { Church }\end{array}$ & $1977-0231$ & & $\begin{array}{c}\text { The Rocks of } \\
\text { Elora }\end{array}$ & 1918/1922? & $\mathrm{cn}$ & $\begin{array}{c}\text { Filmcraft } \\
\text { Industries Ltd. }\end{array}$ & OMPB & & \\
\hline 234380 & $\begin{array}{l}\text { Moravian } \\
\text { Church }\end{array}$ & $1977-0231$ & & Kawartha Lakes & 1922 & $\mathrm{cn}$ & $\begin{array}{l}\text { Pathescope of } \\
\text { Canada }\end{array}$ & OMPB & OMPB & Toned. \\
\hline 234381 & $\begin{array}{l}\text { Moravian } \\
\text { Church }\end{array}$ & $1977-0231$ & & $\begin{array}{c}\text { A Great Lakes } \\
\text { Romance }\end{array}$ & 1918 & $\mathrm{cn}$ & $\begin{array}{c}\text { Exhibits and } \\
\text { Publicity Bureau }\end{array}$ & $\begin{array}{c}\text { Department of } \\
\text { Trade and } \\
\text { Commerce } \\
\end{array}$ & & \\
\hline 234382 & $\begin{array}{l}\text { Moravian } \\
\text { Church }\end{array}$ & $1977-0231$ & $\begin{array}{l}\text { Canadian } \\
\text { National } \\
\text { Pictorial } \\
\end{array}$ & $\begin{array}{c}\text { Hudson's Bay Dog } \\
\text { Derby at Le Pas } \\
\text { [incomplete] }\end{array}$ & 1919/1921? & $\mathrm{cn}$ & $\begin{array}{c}\text { Pathescope of } \\
\text { Canada }\end{array}$ & & OMPB & $\begin{array}{l}\text { Tinted } \\
\text { yellow }\end{array}$ \\
\hline 235800 & $\begin{array}{l}\text { Moravian } \\
\text { Church }\end{array}$ & $1977-0231$ & & $\begin{array}{c}\text { Guardians of the } \\
\text { Deep } 1917\end{array}$ & 1918 & us & Daypho-Bray & & & $\begin{array}{c}\text { Tinted } \\
\text { unknown } \\
\text { colour } \\
\end{array}$ \\
\hline 239308 & $\begin{array}{l}\text { Moravian } \\
\text { Church }\end{array}$ & $1977-0231$ & & Comets & 1920/1929? & us & Bray Pictograph & & & \\
\hline 248232 & $\begin{array}{l}\text { Moravian } \\
\text { Church }\end{array}$ & $1977-0231$ & & $\begin{array}{l}\text { Our Bone } \\
\text { Relations }\end{array}$ & 1917 & us & $\begin{array}{c}\text { The Bray } \\
\text { Productions Inc. } \\
\text { Daypho-Bray } \\
\text { Library of } \\
\text { Motion Picture }\end{array}$ & & OMPB & $\begin{array}{l}\text { Tinted } \\
\text { orange }\end{array}$ \\
\hline 275442 & $\begin{array}{l}\text { Moravian } \\
\text { Church }\end{array}$ & $1977-0231$ & $\begin{array}{l}\text { Canadian } \\
\text { National } \\
\text { Pictorial } \\
\end{array}$ & $\begin{array}{l}\text { The Tide of } \\
\text { Immigration }\end{array}$ & 1919/1921? & $\mathrm{cn}$ & $\begin{array}{l}\text { Pathescope of } \\
\text { Canada }\end{array}$ & & & \\
\hline
\end{tabular}




\begin{tabular}{|c|c|c|c|c|c|c|c|c|c|c|}
\hline 279808 & $\begin{array}{c}\text { Moravian } \\
\text { Church }\end{array}$ & $1977-0231$ & & Minaki & $1924 ?$ & $\mathrm{cn}$ & $\begin{array}{c}\text { Pathescope of } \\
\text { Canada }\end{array}$ & OMPB & & $\begin{array}{c}\text { Minaki } \\
\text { summer } \\
\text { resort, at } \\
\text { Big Sand } \\
\text { Lake, } \\
\text { Ontario. }\end{array}$ \\
\hline 302187 & $\begin{array}{c}\text { Moravian } \\
\text { Church }\end{array}$ & $1977-0231$ & & The Twin Cities & $1922 ?$ & $\mathrm{cn}$ & $\begin{array}{c}\text { Filmcraft } \\
\text { Industries Ltd. }\end{array}$ & OMPB & & $\begin{array}{c}\text { Blue and } \\
\text { sepia toned }\end{array}$ \\
\hline 310175 & $\begin{array}{l}\text { Moravian } \\
\text { Church }\end{array}$ & $1977-0231$ & $\begin{array}{l}\text { Paramount } \\
\text { Pictographs }\end{array}$ & $\begin{array}{l}\text { Better Babies } \\
\text { Character } \\
\text { Building }\end{array}$ & $1920 / 1927 ?$ & us & Paramount & $\begin{array}{l}\text { Woman's home } \\
\text { companion }\end{array}$ & OMPB & $\begin{array}{l}\text { Tinted } \\
\text { unknown } \\
\text { colour }\end{array}$ \\
\hline 310772 & $\begin{array}{l}\text { Moravian } \\
\text { Church }\end{array}$ & $1977-0231$ & & $\begin{array}{c}\text { A Maine } \\
\text { Snowstorm }\end{array}$ & $1917 \mid$ & us & $\begin{array}{c}\text { The Bray } \\
\text { Productions Inc. } \\
\text { Daypho-Bray } \\
\text { Library of } \\
\text { Motion Picture }\end{array}$ & & OMPB & $\begin{array}{l}\text { Tinted } \\
\text { purple }\end{array}$ \\
\hline 337100 & $\begin{array}{c}\text { Moravian } \\
\text { Church }\end{array}$ & $1977-0231$ & & $\begin{array}{c}\text { Ottawa Canada } \\
\text { "The Edinburgh of } \\
\text { North America" : } \\
\text { [incomplete] }\end{array}$ & 1919-11-18 & $\mathrm{cn}$ & $\begin{array}{c}\text { Exhibits and } \\
\text { Publicity Bureau }\end{array}$ & & & $\begin{array}{c}\text { Tinted } \\
\text { unknown } \\
\text { colour } \\
\end{array}$ \\
\hline 368368 & $\begin{array}{c}\text { Moravian } \\
\text { Church }\end{array}$ & $1977-0231$ & & $\begin{array}{l}\text { Studies in } \\
\text { Character } \\
\text { Analysis }\end{array}$ & 1918 & us & $\begin{array}{l}\text { The Bray } \\
\text { Productions Inc. }\end{array}$ & & & $\begin{array}{c}\text { Tinted } \\
\text { unknown } \\
\text { colour } \\
\end{array}$ \\
\hline 379542 & $\begin{array}{c}\text { Moravian } \\
\text { Church }\end{array}$ & $1977-0231$ & $\begin{array}{l}\text { Seeing } \\
\text { Canada }\end{array}$ & $\begin{array}{c}\text { The Queen of the } \\
\text { Coast }\end{array}$ & 1920 & $\mathrm{cn}$ & $\begin{array}{c}\text { Exhibits and } \\
\text { Publicity Bureau }\end{array}$ & $\begin{array}{c}\text { Department of } \\
\text { Trade and } \\
\text { Commerce }\end{array}$ & & \\
\hline 386619 & $\begin{array}{l}\text { Moravian } \\
\text { Church }\end{array}$ & $1977-0231$ & & $\begin{array}{l}\text { The Glens of } \\
\text { Elora }\end{array}$ & $1922 ?$ & $\mathrm{cn}$ & $\begin{array}{l}\text { Pathescope of } \\
\text { Canada }\end{array}$ & OMPB & & $\begin{array}{l}\text { Tinted } \\
\text { yellow }\end{array}$ \\
\hline 385531 & $\begin{array}{c}\text { Moravian } \\
\text { Church }\end{array}$ & $1977-0231$ & & The Triangle Tour & 1920/1929? & $\mathrm{cn}$ & N/A & & & $\begin{array}{l}\text { "scenic tour } \\
\text { along the } \\
\text { Fraser and } \\
\text { Thompson } \\
\text { Rivers, with } \\
\text { a stop along } \\
\text { the way at } \\
\text { an Indian } \\
\text { reserve." } \\
\text { Archivist } \\
\text { note } \\
\end{array}$ \\
\hline 385544 & $\begin{array}{c}\text { Moravian } \\
\text { Church }\end{array}$ & $1977-0231$ & $\begin{array}{l}\text { Canadian } \\
\text { National } \\
\text { Pictorial } \\
\end{array}$ & $\begin{array}{c}\text { The Rainmakers } \\
\text { of the Prairies }\end{array}$ & 1919/1921? & $\mathrm{cn}$ & $\begin{array}{c}\text { Pathescope of } \\
\text { Canada }\end{array}$ & & OMPB & $\begin{array}{c}\text { Tinted } \\
\text { unknown } \\
\text { colour } \\
\end{array}$ \\
\hline 385545 & $\begin{array}{c}\text { Moravian } \\
\text { Church }\end{array}$ & $1977-0231$ & $\begin{array}{l}\text { Canadian } \\
\text { National } \\
\text { Pictorial } \\
\end{array}$ & $\begin{array}{c}\text { Alberta Mormons } \\
\text { Build Magnificent } \\
\text { Temple } \\
\end{array}$ & 1919/1921? & $\mathrm{cn}$ & $\begin{array}{c}\text { Pathescope of } \\
\text { Canada }\end{array}$ & & & $\begin{array}{c}\text { Tinted } \\
\text { unknown } \\
\text { colour } \\
\end{array}$ \\
\hline 385546 & $\begin{array}{c}\text { Moravian } \\
\text { Church }\end{array}$ & $1977-0231$ & $\begin{array}{l}\text { Canadian } \\
\text { National } \\
\text { Pictorial } \\
\end{array}$ & $\begin{array}{l}\text { Higher Education } \\
\text { in the West }\end{array}$ & 1919/1921? & $\mathrm{cn}$ & $\begin{array}{l}\text { Pathescope of } \\
\text { Canada }\end{array}$ & & & $\begin{array}{c}\text { Tinted } \\
\text { unknown } \\
\text { colour } \\
\end{array}$ \\
\hline 385547 & $\begin{array}{c}\text { Moravian } \\
\text { Church }\end{array}$ & $1977-0231$ & $\begin{array}{l}\text { Canadian } \\
\text { National } \\
\text { Pictorial } \\
\end{array}$ & $\begin{array}{c}\text { Good Bye to the } \\
\text { Hello Girls }\end{array}$ & 1919/1921? & $\mathrm{cn}$ & $\begin{array}{c}\text { Pathescope of } \\
\text { Canada }\end{array}$ & & & $\begin{array}{c}\text { Tinted } \\
\text { unknown } \\
\text { colour } \\
\end{array}$ \\
\hline 385560 & $\begin{array}{c}\text { Moravian } \\
\text { Church }\end{array}$ & $1977-0231$ & $\begin{array}{l}\text { Canadian } \\
\text { National } \\
\text { Pictorial } \\
\end{array}$ & $\begin{array}{c}\text { Canada's Harvest } \\
\text { of Fish }\end{array}$ & 1919 & $\mathrm{cn}$ & $\begin{array}{l}\text { Pathescope of } \\
\text { Canada }\end{array}$ & & & $\begin{array}{l}\text { Tinted } \\
\text { yellow }\end{array}$ \\
\hline 386310 & $\begin{array}{c}\text { Moravian } \\
\text { Church }\end{array}$ & $1977-0231$ & & $\begin{array}{l}\text { Land Battleships } \\
\text { in Private Life }\end{array}$ & 1920/1925? & N/A & $\mathrm{N} / \mathrm{A}$ & & & $\begin{array}{c}\text { Tinted } \\
\text { unknown } \\
\text { colour } \\
\end{array}$ \\
\hline 386621 & $\begin{array}{c}\text { Moravian } \\
\text { Church }\end{array}$ & $1977-0231$ & $\begin{array}{l}\text { Seeing } \\
\text { Canada }\end{array}$ & $\begin{array}{c}\text { Canada's } \\
\text { Metropolis }\end{array}$ & 1921/1922? & $\mathrm{cn}$ & $\begin{array}{c}\text { Exhibits and } \\
\text { Publicity Bureau }\end{array}$ & $\begin{array}{c}\text { Department of } \\
\text { Trade and } \\
\text { Commerce }\end{array}$ & & $\begin{array}{l}\text { Tinted } \\
\text { yellow }\end{array}$ \\
\hline 386622 & $\begin{array}{c}\text { Moravian } \\
\text { Church }\end{array}$ & $1977-0231$ & $\begin{array}{c}\text { Pathé } \\
\text { Review }\end{array}$ & $\begin{array}{l}\text { The High and } \\
\text { Broad Jump }\end{array}$ & $1920 / 1925 ?$ & $\mathrm{cn}$ & $\begin{array}{c}\text { Novagraph Film } \\
\text { Company }\end{array}$ & & & \\
\hline 386624 & $\begin{array}{c}\text { Moravian } \\
\text { Church }\end{array}$ & $1977-0231$ & & $\begin{array}{l}\text { Prayer Time at the } \\
\text { Great Mosque }\end{array}$ & 1914/1924? & $\mathrm{fr}$ & Pathe Freres & & & $\begin{array}{c}\text { Notes says. } \\
\text { Believed to } \\
\text { be an } \\
\text { OMPB film }\end{array}$ \\
\hline 386625 & $\begin{array}{c}\text { Moravian } \\
\text { Church }\end{array}$ & $1977-0231$ & & $\begin{array}{c}\text { The Rice Harvest } \\
\text { in Japan }\end{array}$ & 1920/1924? & $\mathrm{fr}$ & Pathe Freres & & & \\
\hline 386626 & $\begin{array}{c}\text { Moravian } \\
\text { Church }\end{array}$ & $1977-0231$ & & $\begin{array}{c}\text { Belgian Cavalry } \\
\text { on a Cross } \\
\text { Country Ride } \\
\end{array}$ & 1919/1924? & N/A & Pathe Freres & & & \\
\hline
\end{tabular}




\begin{tabular}{|c|c|c|c|c|c|c|c|c|c|}
\hline 386627 & $\begin{array}{c}\text { Moravian } \\
\text { Church }\end{array}$ & $1977-0231$ & $\begin{array}{l}\text { Seeing } \\
\text { Canada }\end{array}$ & $\begin{array}{c}\text { Through the } \\
\text { Norway of } \\
\text { America } \\
\end{array}$ & 1920 & $\mathrm{cn}$ & $\begin{array}{c}\text { Exhibits and } \\
\text { Publicity Bureau }\end{array}$ & $\begin{array}{c}\text { Department of } \\
\text { Trade and } \\
\text { Commerce } \\
\end{array}$ & \\
\hline 386628 & $\begin{array}{l}\text { Moravian } \\
\text { Church }\end{array}$ & $1977-0231$ & & $\begin{array}{l}\text { Arts and Crafts of } \\
\text { India }\end{array}$ & 1920/1924? & $\mathrm{fr}$ & Pathe Freres & & \\
\hline 386630 & $\begin{array}{c}\text { Moravian } \\
\text { Church }\end{array}$ & $1977-0231$ & & $\begin{array}{c}\text { The Rival of } \\
\text { Daylight }\end{array}$ & 1919 & N/A & $\begin{array}{l}\text { Pathescope of } \\
\text { Canada }\end{array}$ & & $\begin{array}{l}\text { Tinted } \\
\text { unknown } \\
\text { colour } \\
\end{array}$ \\
\hline 386631 & $\begin{array}{l}\text { Moravian } \\
\text { Church }\end{array}$ & $1977-0231$ & & Colombo & $1919 / 1921$ & $\mathrm{cn}$ & $\begin{array}{l}\text { Pathescope of } \\
\text { Canada }\end{array}$ & & \\
\hline 386632 & $\begin{array}{c}\text { Moravian } \\
\text { Church } \\
\end{array}$ & $1977-0231$ & & $\begin{array}{c}\text { Mechanical } \\
\text { Operation of } \\
\text { British Tanks } \\
\end{array}$ & 1919/1924? & us & $\begin{array}{c}\text { Bray } \\
\text { Productions Inc. }\end{array}$ & & \\
\hline 386633 & $\begin{array}{c}\text { Moravian } \\
\text { Church }\end{array}$ & 1977-0231 & & $\begin{array}{c}\text { The Guns of Our } \\
\text { Forefathers }\end{array}$ & 1919/1924? & us & $\begin{array}{c}\text { Bray } \\
\text { Productions Inc. }\end{array}$ & & $\begin{array}{l}\text { Tinted } \\
\text { unknown } \\
\text { colour } \\
\end{array}$ \\
\hline 386634 & $\begin{array}{c}\text { Moravian } \\
\text { Church }\end{array}$ & 1977-0231 & $\begin{array}{l}\text { Canadian } \\
\text { National } \\
\text { Pictorial } \\
\end{array}$ & $\begin{array}{c}\text { Mounted Sports at } \\
\text { R.M.C. } \\
\end{array}$ & $1919 / 1921$ & $\mathrm{cn}$ & $\begin{array}{l}\text { Pathescope of } \\
\text { Canada }\end{array}$ & $\begin{array}{c}\text { Exhibits and } \\
\text { Publicity Bureau }\end{array}$ & $\begin{array}{l}\text { Tinted } \\
\text { yellow }\end{array}$ \\
\hline 386637 & $\begin{array}{c}\text { Moravian } \\
\text { Church }\end{array}$ & $1977-0231$ & $\begin{array}{l}\text { Canadian } \\
\text { National } \\
\text { Pictorial } \\
\end{array}$ & $\begin{array}{c}\text { Keeping Canadian } \\
\text { Home Fires } \\
\text { Burning } \\
\end{array}$ & $1919 / 1921$ & $\mathrm{cn}$ & $\begin{array}{l}\text { Pathescope of } \\
\text { Canada }\end{array}$ & & $\begin{array}{l}\text { Tinted } \\
\text { Yellow } \\
\end{array}$ \\
\hline 386638 & $\begin{array}{c}\text { Moravian } \\
\text { Church } \\
\end{array}$ & $1977-0231$ & & $\begin{array}{c}\text { Ontario } \\
\text { Experimental } \\
\text { Farms } \\
\end{array}$ & $1922 ?$ & $\mathrm{cn}$ & $\begin{array}{l}\text { Pathescope of } \\
\text { Canada }\end{array}$ & OMPB & $\begin{array}{l}\text { Tinted } \\
\text { yellow }\end{array}$ \\
\hline 387274 & $\begin{array}{l}\text { Moravian } \\
\text { Church }\end{array}$ & $1977-0231$ & & $\begin{array}{l}\text { A Normandy } \\
\text { Market }\end{array}$ & $1918 / 1924 ?$ & $\mathrm{fr}$ & Pathe Freres & & \\
\hline 387428 & $\begin{array}{c}\text { Moravian } \\
\text { Church }\end{array}$ & $1977-0231$ & & $\begin{array}{c}\text { The Romance of } \\
\text { Crater Lake }\end{array}$ & 1919 & us & $\begin{array}{c}\text { Bray } \\
\text { Productions Inc. }\end{array}$ & & $\begin{array}{l}\text { Tinted } \\
\text { purple }\end{array}$ \\
\hline 413335 & $\begin{array}{c}\text { Moravian } \\
\text { Church }\end{array}$ & 1977-0231 & & Ontario & 1927 & $\mathrm{cn}$ & $\begin{array}{l}\text { Ontario Motion } \\
\text { Picture Bureau }\end{array}$ & & \\
\hline 413605 & $\begin{array}{c}\text { Moravian } \\
\text { Church }\end{array}$ & $1977-0231$ & & $\begin{array}{c}\text { America's } \\
\text { Sleeping Giant } \\
\end{array}$ & $1920 / 1929 ?$ & us & $\begin{array}{c}\text { Bray } \\
\text { Productions Inc. }\end{array}$ & & $\begin{array}{l}\text { Tinted } \\
\text { Green } \\
\end{array}$ \\
\hline 413608 & $\begin{array}{c}\text { Moravian } \\
\text { Church }\end{array}$ & $1977-0231$ & & $\begin{array}{l}\text { A Lesson in } \\
\text { Woodcarving }\end{array}$ & 1920/1924? & us & $\begin{array}{c}\text { Bray } \\
\text { Productions Inc. }\end{array}$ & & $\begin{array}{c}\text { Tinted } \\
\text { unknown } \\
\text { colour } \\
\end{array}$ \\
\hline 415123 & $\begin{array}{c}\text { Moravian } \\
\text { Church }\end{array}$ & $1977-0231$ & & $\begin{array}{c}\text { Picturesque } \\
\text { Naples } \\
\end{array}$ & 1920/1924? & $\mathrm{fr}$ & Pathe Freres & & \\
\hline 415142 & $\begin{array}{c}\text { Moravian } \\
\text { Church }\end{array}$ & $1977-0231$ & $\begin{array}{l}\text { Paramount } \\
\text { Pictograph }\end{array}$ & $\begin{array}{c}\text { Discovery of the } \\
\text { Shot Tower }\end{array}$ & 1920/1924? & us & Paramount & & $\begin{array}{l}\text { Tinted } \\
\text { unknown } \\
\text { colour } \\
\end{array}$ \\
\hline 415143 & $\begin{array}{c}\text { Moravian } \\
\text { Church }\end{array}$ & $1977-0231$ & & $\begin{array}{c}\text { An Ocean } \\
\text { Paradise } \\
\end{array}$ & 1920/1924? & us & $\begin{array}{l}\text { Bray Studios } \\
\text { Inc. } \\
\end{array}$ & & \\
\hline 415144 & $\begin{array}{c}\text { Moravian } \\
\text { Church }\end{array}$ & $1977-0231$ & & $\begin{array}{l}\text { Harnessing the } \\
\text { Wind } \\
\end{array}$ & 1920/1930? & us & $\begin{array}{c}\text { Goldwyn Bray } \\
\text { Pictographs } \\
\end{array}$ & & Tinted pink \\
\hline 415146 & $\begin{array}{c}\text { Moravian } \\
\text { Church }\end{array}$ & $1977-0231$ & & $\begin{array}{l}\text { Putting the Nut to } \\
\text { Work }\end{array}$ & 1920/1930? & us & Paramount & & $\begin{array}{c}\text { Tinted } \\
\text { unknown } \\
\text { colour }\end{array}$ \\
\hline 415147 & $\begin{array}{c}\text { Moravian } \\
\text { Church }\end{array}$ & $1977-0231$ & & $\begin{array}{c}\text { Submarine } \\
\text { Gardens }\end{array}$ & 1920/1930? & us & Paramount & & $\begin{array}{c}\text { Tinted } \\
\text { orange }\end{array}$ \\
\hline 415195 & $\begin{array}{c}\text { Moravian } \\
\text { Church }\end{array}$ & $1977-0231$ & & $\begin{array}{l}\text { Ice Effects at } \\
\text { Odessa Russia } \\
\end{array}$ & 1920/1924? & $\mathrm{fr}$ & Pathe Freres & & \\
\hline 415196 & $\begin{array}{c}\text { Moravian } \\
\text { Church }\end{array}$ & $1977-0231$ & & Hydrogen & 1920/1924? & $\mathrm{fr}$ & Pathe Freres & & \\
\hline 415197 & $\begin{array}{c}\text { Moravian } \\
\text { Church }\end{array}$ & $1977-0231$ & $\begin{array}{c}\text { Pathé } \\
\text { Review }\end{array}$ & $\begin{array}{l}\text { An Enemy of } \\
\text { Snakes }\end{array}$ & 1919/1927? & us & Pathe Freres & & $\begin{array}{c}\text { unknowns } \\
\text { country }\end{array}$ \\
\hline 415198 & $\begin{array}{c}\text { Moravian } \\
\text { Church }\end{array}$ & $1977-0231$ & & $\begin{array}{c}\text { Catching and } \\
\text { Canning Tunny- } \\
\text { Fish Sicily } \\
\end{array}$ & $1920 / 1924 ?$ & $\mathrm{fr}$ & Pathe Freres & & \\
\hline 415199 & $\begin{array}{c}\text { Moravian } \\
\text { Church }\end{array}$ & $1977-0231$ & & $\begin{array}{c}\text { Mushroom } \\
\text { Growing }\end{array}$ & 1920/1924? & $\mathrm{fr}$ & Pathe Freres & & \\
\hline 415200 & $\begin{array}{c}\text { Moravian } \\
\text { Church } \\
\end{array}$ & $1977-0231$ & $\begin{array}{c}\text { Goldwyn } \\
\text { Bray } \\
\text { Pictographs } \\
\end{array}$ & $\begin{array}{l}\text { Making the Dirt } \\
\text { Fly }\end{array}$ & 1920/1930? & us & Goldwyn-Bray & & $\begin{array}{l}\text { Tinted } \\
\text { unknown } \\
\text { colour } \\
\end{array}$ \\
\hline 415202 & $\begin{array}{c}\text { Moravian } \\
\text { Church }\end{array}$ & $1977-0231$ & & $\begin{array}{l}\text { Gathering and } \\
\text { Preparing } \\
\text { Cocoanuts in the } \\
\text { Philippines }\end{array}$ & 1920/1924? & $\mathrm{fr}$ & Pathe Freres & & \\
\hline 415203 & $\begin{array}{c}\text { Moravian } \\
\text { Church }\end{array}$ & $1977-0231$ & & $\begin{array}{c}\text { Experiments With } \\
\text { Light Rays }\end{array}$ & 1920/1930? & us & $\begin{array}{c}\text { Bray } \\
\text { Productions Inc. }\end{array}$ & & \\
\hline 415204 & Moravian & $1977-0231$ & & Helping the Deaf & $1920 / 1924 ?$ & us & Bray & & Tinted \\
\hline
\end{tabular}




\begin{tabular}{|c|c|c|c|c|c|c|c|c|c|c|}
\hline & Church & & & to Hear & & & Productions Inc. & & & orange \\
\hline 415206 & $\begin{array}{c}\text { Moravian } \\
\text { Church }\end{array}$ & $1977-0231$ & & $\begin{array}{c}\text { An Old-Fashioned } \\
\text { Coon Hunt }\end{array}$ & 1920/1927? & us & $\begin{array}{c}\text { Bray } \\
\text { Productions Inc. }\end{array}$ & & & Tinted blue \\
\hline 415207 & $\begin{array}{c}\text { Moravian } \\
\text { Church }\end{array}$ & $1977-0231$ & & Pup Portraits & $1920 / 1930 ?$ & us & $\begin{array}{c}\text { Goldwyn-Bray } \\
\text { Productions }\end{array}$ & & & Tinted pink. \\
\hline 415208 & $\begin{array}{c}\text { Moravian } \\
\text { Church }\end{array}$ & $1977-0231$ & & Slate Industry & 1920/1924? & $\mathrm{fr}$ & Pathe Freres & & & \\
\hline 415209 & $\begin{array}{c}\text { Moravian } \\
\text { Church }\end{array}$ & $1977-0231$ & $\begin{array}{l}\text { Paramount } \\
\text { Pictograms } \\
\end{array}$ & $\begin{array}{c}\text { Scenic Gems from } \\
\text { Italy }\end{array}$ & $1920 / 1930 ?$ & us & Paramount & & & $\begin{array}{r}\text { Tinted } \\
\text { yellow }\end{array}$ \\
\hline 415210 & $\begin{array}{c}\text { Moravian } \\
\text { Church }\end{array}$ & $1977-0231$ & & $\begin{array}{c}\text { Tea Industry in } \\
\text { Japan }\end{array}$ & 1920/1930? & us & $\begin{array}{c}\text { Bray } \\
\text { Productions Inc. }\end{array}$ & & & $\begin{array}{c}\text { Tinted } \\
\text { Orange } \\
\end{array}$ \\
\hline 415211 & $\begin{array}{c}\text { Moravian } \\
\text { Church }\end{array}$ & $1977-0231$ & & $\begin{array}{c}\text { Tea Gathering and } \\
\text { Preparation } \\
\end{array}$ & 1920/1924? & N/A & Pathe Freres & & OMPB & \\
\hline 415212 & $\begin{array}{c}\text { Moravian } \\
\text { Church }\end{array}$ & 1977-0231 & & $\begin{array}{c}\text { A Trip to the } \\
\text { Island of Majorca }\end{array}$ & $1920 / 1925 ?$ & N/A & Pathe Freres & & & \\
\hline 415213 & $\begin{array}{l}\text { Moravian } \\
\text { Church }\end{array}$ & $1977-0231$ & & $\begin{array}{c}\text { Treasures of a } \\
\text { Great City : } \\
\text { History of the } \\
\text { Book }\end{array}$ & 1918 & us & $\begin{array}{c}\text { Bray } \\
\text { Productions Inc. }\end{array}$ & & & $\begin{array}{l}\text { Tinted } \\
\text { yellow }\end{array}$ \\
\hline 415214 & $\begin{array}{c}\text { Moravian } \\
\text { Church } \\
\end{array}$ & $1977-0231$ & & $\begin{array}{c}\text { The Passing of the } \\
\text { Cannibal }\end{array}$ & 1920/1930? & us & $\begin{array}{c}\text { Bray } \\
\text { Productions Inc. }\end{array}$ & & & $\begin{array}{l}\text { Tinted } \\
\text { yellow }\end{array}$ \\
\hline 415215 & $\begin{array}{c}\text { Moravian } \\
\text { Church }\end{array}$ & $1977-0231$ & & $\begin{array}{c}\text { Forestry in } \\
\text { Australia }\end{array}$ & $1920 / 1924 ?$ & $\mathrm{fr}$ & Pathe Freres & & & \\
\hline 415216 & $\begin{array}{c}\text { Moravian } \\
\text { Church }\end{array}$ & $1977-0231$ & & $\begin{array}{c}\text { Manufacturing } \\
\text { Corks in Scotland } \\
\end{array}$ & $1920 / 1924 ?$ & $\mathrm{fr}$ & Pathe Freres & & & \\
\hline 415217 & $\begin{array}{c}\text { Moravian } \\
\text { Church }\end{array}$ & $1977-0231$ & $\begin{array}{l}\text { The } \\
\text { Magazine on } \\
\text { the Screen }\end{array}$ & How We Hear & 1920 & us & $\begin{array}{c}\text { Bray } \\
\text { Productions Inc. }\end{array}$ & & & Tinted red \\
\hline 415218 & $\begin{array}{c}\text { Moravian } \\
\text { Church }\end{array}$ & $1977-0231$ & & $\begin{array}{c}\text { The Bee and the } \\
\text { Rose }\end{array}$ & 1920/1924? & $\mathrm{fr}$ & Pathe Freres & & & \\
\hline 415219 & $\begin{array}{c}\text { Moravian } \\
\text { Church }\end{array}$ & $1977-0231$ & & $\begin{array}{l}\text { Nara the Park of } \\
\text { the Sacred Hind }\end{array}$ & $1920 / 1924 ?$ & $\mathrm{fr}$ & Pathe Freres & & & \\
\hline 415220 & $\begin{array}{c}\text { Moravian } \\
\text { Church }\end{array}$ & $1977-0231$ & $\begin{array}{c}\text { Daypho- } \\
\text { Bray } \\
\text { Pictograph } \\
\end{array}$ & $\begin{array}{c}\text { Putting Volcanoes } \\
\text { to Work }\end{array}$ & 1917 & us & $\begin{array}{c}\text { Bray Studios } \\
\text { Inc. } \\
\end{array}$ & $\begin{array}{c}\text { Popular Science } \\
\text { Weekly }\end{array}$ & & \\
\hline 415221 & $\begin{array}{c}\text { Moravian } \\
\text { Church }\end{array}$ & $1977-0231$ & & $\begin{array}{l}\text { Removing a } \\
\text { Mountain }\end{array}$ & $1920 / 1924 ?$ & $\mathrm{fr}$ & Pathe Freres & & & \\
\hline 415222 & $\begin{array}{c}\text { Moravian } \\
\text { Church }\end{array}$ & $1977-0231$ & & $\begin{array}{c}\text { Ruins of Wisby A } \\
\text { Swedish Village } \\
\text { on the East Coast } \\
\text { of the Island of } \\
\text { Gothland }\end{array}$ & 1920/1924? & $\mathrm{fr}$ & Pathe Freres & & & \\
\hline 415223 & $\begin{array}{c}\text { Moravian } \\
\text { Church }\end{array}$ & $1977-0231$ & $\begin{array}{c}\text { Ashley } \\
\text { Miller } \\
\text { Plastique } \\
\end{array}$ & Gravitation & 1916 & us & $\begin{array}{c}\text { Bray } \\
\text { Productions Inc. }\end{array}$ & & & \\
\hline 415224 & $\begin{array}{c}\text { Moravian } \\
\text { Church }\end{array}$ & $1977-0231$ & & $\begin{array}{l}\text { The Black Sea in } \\
\text { Stormy Weather }\end{array}$ & 1920/1924? & fr & Pathe Freres & & & \\
\hline 415225 & $\begin{array}{c}\text { Moravian } \\
\text { Church }\end{array}$ & 1977-0231 & & Among the Roses & $1924 / 1929 ?$ & $\mathrm{fr}$ & Pathe Freres & & OMPB & \\
\hline 415226 & $\begin{array}{c}\text { Moravian } \\
\text { Church }\end{array}$ & $1977-0231$ & & The Ant-Lion & 1920/1924? & N/A & Pathe Freres & & & \\
\hline 415227 & $\begin{array}{c}\text { Moravian } \\
\text { Church }\end{array}$ & 1977-0231 & $\begin{array}{c}\text { Pathé } \\
\text { Review } \\
\end{array}$ & $\begin{array}{c}\text { The Wall Street of } \\
\text { Antville }\end{array}$ & $1919 / 1927$ & us & Pathe Freres & & & \\
\hline 415228 & $\begin{array}{c}\text { Moravian } \\
\text { Church }\end{array}$ & $1977-0231$ & $\begin{array}{c}\text { Pathé } \\
\text { Review }\end{array}$ & $\begin{array}{c}\text { Underneath the } \\
\text { Trap Door -- A } \\
\text { Little Call on a } \\
\text { Lady Spider } \\
\end{array}$ & 1919/1927? & us & Pathe Freres & & & \\
\hline 415229 & $\begin{array}{c}\text { Moravian } \\
\text { Church }\end{array}$ & 1977-0231 & & Views of Russia & 1920/1924? & $\mathrm{fr}$ & Pathe Freres & & & \\
\hline 415230 & $\begin{array}{c}\text { Moravian } \\
\text { Church }\end{array}$ & $1977-0231$ & & $\begin{array}{c}\text { The Twentieth } \\
\text { Chasseurs } \\
\text { Exercising Horses }\end{array}$ & 1920/1924? & $\mathrm{fr}$ & Pathe Freres & & & \\
\hline 415231 & $\begin{array}{c}\text { Moravian } \\
\text { Church }\end{array}$ & $1977-0231$ & & $\begin{array}{c}\text { Ophiures : } \\
\text { Oceanography } \\
\end{array}$ & 1920/1924? & N/A & Pathe Freres & & & \\
\hline 415232 & $\begin{array}{c}\text { Moravian } \\
\text { Church }\end{array}$ & 1977-0231 & $\begin{array}{c}\text { Goldwyn- } \\
\text { Bray } \\
\text { Pictographs }\end{array}$ & Nice A Dan Banan & $1920 / 1924 ?$ & us & Goldwyn-Bray & & & $\begin{array}{c}\text { Tinted } \\
\text { Orange }\end{array}$ \\
\hline 415233 & $\begin{array}{c}\text { Moravian } \\
\text { Church }\end{array}$ & 1977-0231 & & $\begin{array}{c}\text { Jelly Fish : } \\
\text { [incomplete] }\end{array}$ & 1918/1924? & N/A & Pathe Freres & & & \\
\hline
\end{tabular}




\begin{tabular}{|c|c|c|c|c|c|c|c|c|c|c|}
\hline 415235 & $\begin{array}{c}\text { Moravian } \\
\text { Church }\end{array}$ & $1977-0231$ & & $\begin{array}{c}\text { Ingenious Insects : } \\
\text { The Trapdoor } \\
\text { Spider } \\
\end{array}$ & 1918 & us & $\begin{array}{c}\text { Bray } \\
\text { Productions Inc. }\end{array}$ & & & $\begin{array}{l}\text { Tinted } \\
\text { yellow }\end{array}$ \\
\hline 415246 & $\begin{array}{l}\text { Moravian } \\
\text { Church }\end{array}$ & $1977-0231$ & & Strasburg & 1920/1924? & fr & Pathe Freres & & & \\
\hline 415771 & $\begin{array}{l}\text { Moravian } \\
\text { Church }\end{array}$ & $1977-0231$ & & A Trip to Kabyle & 1920/1924? & fr & Pathe Freres & & & \\
\hline 416323 & $\begin{array}{l}\text { Moravian } \\
\text { Church }\end{array}$ & $1977-0231$ & & $\begin{array}{l}\text { The Carrot } \\
\text { Caterpillar } \\
\end{array}$ & 1920/1929? & N/A & Pathe Freres & & & \\
\hline 435537 & $\begin{array}{l}\text { Moravian } \\
\text { Church }\end{array}$ & $1977-0231$ & $\begin{array}{l}\text { Goldwyn- } \\
\text { Bray } \\
\text { Pictograph }\end{array}$ & A Dog Musician & 1920 & us & Goldwyn-Bray & & OMPB & $\begin{array}{c}\text { Tinted } \\
\text { unknown } \\
\text { colour }\end{array}$ \\
\hline 435573 & $\begin{array}{l}\text { Moravian } \\
\text { Church }\end{array}$ & $1977-0231$ & & Max Goes Skating & 1907 & fr & Pathe Freres & & & $\begin{array}{c}\text { Stars Max } \\
\text { Linder }\end{array}$ \\
\hline 435574 & $\begin{array}{l}\text { Moravian } \\
\text { Church }\end{array}$ & $1977-0231$ & & $\begin{array}{c}\text { [Unidentified Bray } \\
\text { Studios Film] }\end{array}$ & 1918/1928? & us & $\begin{array}{l}\text { Bray Studios } \\
\text { Inc. }\end{array}$ & & & $\begin{array}{c}\text { Tinted } \\
\text { unknown } \\
\text { colour } \\
\end{array}$ \\
\hline 439108 & $\begin{array}{l}\text { Moravian } \\
\text { Church }\end{array}$ & $1977-0231$ & & The Frog & 1920/1924? & N/A & Pathe Freres & & & \\
\hline 464301 & $\begin{array}{l}\text { Moravian } \\
\text { Church }\end{array}$ & $1977-0231$ & & $\begin{array}{l}\text { Removing A } \\
\text { Mountain }\end{array}$ & 1920/1924? & fr & Pathe Freres & & & \\
\hline 464391 & $\begin{array}{l}\text { Moravian } \\
\text { Church }\end{array}$ & $1977-0231$ & $\begin{array}{l}\text { Seeing } \\
\text { Canada }\end{array}$ & $\begin{array}{l}\text { Through the } \\
\text { Norway of } \\
\text { America }\end{array}$ & 1920 & $\mathrm{cn}$ & $\begin{array}{c}\text { Exhibits and } \\
\text { Publicity Bureau }\end{array}$ & $\begin{array}{c}\text { Department of } \\
\text { Trade and } \\
\text { Commerce }\end{array}$ & & $\begin{array}{c}\text { Tinted and } \\
\text { toned } \\
\text { unknown } \\
\text { colour }\end{array}$ \\
\hline 476558 & $\begin{array}{l}\text { Moravian } \\
\text { Church }\end{array}$ & $1977-0231$ & $\begin{array}{l}\text { Canadian } \\
\text { National } \\
\text { Pictorial }\end{array}$ & Queen of the May & $1919 / 1921$ & $\mathrm{cn}$ & $\begin{array}{c}\text { Pathescope of } \\
\text { Canada }\end{array}$ & $\begin{array}{c}\text { Exhibits and } \\
\text { Publicity Bureau }\end{array}$ & & $\begin{array}{c}\text { Tinted } \\
\text { unknown } \\
\text { colour }\end{array}$ \\
\hline 21471 & $\begin{array}{l}\text { Moravian } \\
\text { Church }\end{array}$ & $1977-0231$ & & $\begin{array}{c}\text { Canada's Men-O- } \\
\text { War } \\
\end{array}$ & 1920/1929? & $\mathrm{cn}$ & N/A & & & $\begin{array}{l}\text { Tinted } \\
\text { yellow }\end{array}$ \\
\hline 21549 & $\begin{array}{c}\text { Moravian } \\
\text { Church }\end{array}$ & $1977-0231$ & $\begin{array}{l}\text { Canadian } \\
\text { National } \\
\text { Pictorial } \\
\end{array}$ & $\begin{array}{c}\text { Police for the Oil } \\
\text { Regions }\end{array}$ & $1919 / 1921$ & $\mathrm{cn}$ & $\begin{array}{c}\text { Pathescope of } \\
\text { Canada }\end{array}$ & & & \\
\hline 21981 & $\begin{array}{l}\text { Moravian } \\
\text { Church }\end{array}$ & $1977-0231$ & $\begin{array}{l}\text { Canadian } \\
\text { National } \\
\text { Pictorial } \\
\end{array}$ & $\begin{array}{l}\text { Claiming the } \\
\text { Colours }\end{array}$ & 1919/1921? & $\mathrm{cn}$ & $\begin{array}{c}\text { Pathescope of } \\
\text { Canada }\end{array}$ & & $\begin{array}{c}\text { Famous } \\
\text { Players Film } \\
\text { Service }\end{array}$ & \\
\hline 26816 & $\begin{array}{l}\text { Moravian } \\
\text { Church }\end{array}$ & $1977-0231$ & & $\begin{array}{c}\text { Toronto : A Great } \\
\text { City }\end{array}$ & 1924 & $\mathrm{cn}$ & $\begin{array}{c}\text { Ontario Motion } \\
\text { Picture Bureau }\end{array}$ & & & \\
\hline 90567 & $\begin{array}{l}\text { Moravian } \\
\text { Church }\end{array}$ & $1977-0231$ & & $\begin{array}{l}\text { The Destructive } \\
\text { Power of T.N.T. }\end{array}$ & 1919/1924? & us & $\begin{array}{c}\text { Bray } \\
\text { Productions Inc. }\end{array}$ & & & $\begin{array}{c}\text { Tinted } \\
\text { unknown } \\
\text { colour } \\
\end{array}$ \\
\hline 95247 & $\begin{array}{l}\text { Moravian } \\
\text { Church }\end{array}$ & $1977-0231$ & $\begin{array}{l}\text { Canadian } \\
\text { National } \\
\text { Pictorial } \\
\end{array}$ & $\begin{array}{c}\text { Honour to Those } \\
\text { Who Fell }\end{array}$ & $1919 / 1921$ & $\mathrm{cn}$ & $\begin{array}{c}\text { Pathescope of } \\
\text { Canada }\end{array}$ & & & $\begin{array}{c}\text { Tinted } \\
\text { unknown } \\
\text { colour }\end{array}$ \\
\hline 95248 & $\begin{array}{l}\text { Moravian } \\
\text { Church }\end{array}$ & $1977-0231$ & $\begin{array}{l}\text { Canadian } \\
\text { National } \\
\text { Pictorial } \\
\end{array}$ & $\begin{array}{c}\text { The Artillery of } \\
\text { Canada's Island } \\
\text { Province }= \\
\text { [Canada's Soldiers } \\
\text { at Home] } \\
\end{array}$ & $1919 / 1921 \mid$ & $\mathrm{cn}$ & $\begin{array}{c}\text { Pathescope of } \\
\text { Canada } \\
\end{array}$ & & & $\begin{array}{l}\text { Tinted } \\
\text { yellow }\end{array}$ \\
\hline & & $\begin{array}{c}\text { Miller, Cecil } \\
\text { J. }\end{array}$ & & & & & & & & \\
\hline 327553 & Miller, Cecil J. & $1980-0196$ & & $\begin{array}{c}\text { [On Lake } \\
\text { Superior]: } \\
\text { incomplete }\end{array}$ & $1929 ?$ & $\mathrm{cn}$ & OMPB & & & \\
\hline & & \multicolumn{2}{|c|}{$\begin{array}{c}\text { Canadian Broadcasting } \\
\text { Corporation }\end{array}$} & & & & & & & \\
\hline 188461 & $\mathrm{cbc}$ & 1981-0156 & $\begin{array}{c}\text { The World's } \\
\text { Greatest } \\
\text { Story }\end{array}$ & $\begin{array}{l}\text { Episode 14: } \\
\text { incomplete }\end{array}$ & 1919 & uk & New Era Film & $\begin{array}{c}\text { War Office } \\
\text { Official }\end{array}$ & $\begin{array}{c}\text { Ashley } \\
\text { Exclusive } \\
\text { Films } \\
\end{array}$ & \\
\hline 220737 & $\mathrm{cbc}$ & 1981-0156 & $\begin{array}{c}\text { Canadian } \\
\text { War Records }\end{array}$ & $\begin{array}{l}\text { The Surgical } \\
\text { Motor Ambulance }\end{array}$ & $1915 ?$ & $\mathrm{cn}$ & $\begin{array}{c}\text { Pathescope of } \\
\text { Canada } \\
\end{array}$ & & $\begin{array}{c}\text { Province of } \\
\text { Ontario } \\
\text { Pictures } \\
\end{array}$ & \\
\hline 234631 & $\mathrm{cbc}$ & 1981-0156 & & $\begin{array}{c}\text { Ontario VCs } \\
\text { Leave for London }\end{array}$ & 1919 & $\mathrm{cn}$ & N/A & & & \\
\hline 239309 & $\mathrm{cbc}$ & 1981-0156 & & $\begin{array}{l}\text { [Britain's Air } \\
\text { Force] }\end{array}$ & N/A & N/A & Pathe Freres & & & \\
\hline 279822 & $\mathrm{cbc}$ & $1981-0156$ & & $\begin{array}{c}\text { Visit of Canadian } \\
\text { War Orphans to } \\
\text { Vimy Ridge = The } \\
\text { Orphans of Vimy } \\
\text { Ridge }\end{array}$ & 1925 & cn & $\begin{array}{l}\text { Ontario Motion } \\
\text { Picture Bureau }\end{array}$ & & & \\
\hline
\end{tabular}




\begin{tabular}{|c|c|c|c|c|c|c|c|c|c|c|}
\hline 284351 & $\mathrm{cbc}$ & 1981-0156 & & $\begin{array}{c}\text { Scarborough Golf } \\
\text { Course : } \\
\text { incomplete] } \\
\end{array}$ & 1920/1929? & $\mathrm{cn}$ & N/A & & & \\
\hline 284352 & $\mathrm{cbc}$ & 1981-0156 & & $\begin{array}{c}\text { The Hindenburg } \\
\text { Line : } \\
\text { [incomplete] } \\
1918 \\
\end{array}$ & 1919 & $\mathrm{uk}$ & New Era Film & $\begin{array}{c}\text { War Office } \\
\text { Official } \\
\end{array}$ & & \\
\hline 284392 & $\mathrm{cbc}$ & 1981-0156 & & $\begin{array}{c}\text { With the Canadian } \\
\text { Force: } \\
\text { [incomplete] }\end{array}$ & 1917 & $\mathrm{uk}$ & $\begin{array}{c}\text { Topical Film } \\
\text { Company }\end{array}$ & $\begin{array}{c}\text { War Office } \\
\text { Cinema } \\
\text { Committee for } \\
\text { Canadian War } \\
\text { Records Office } \\
\end{array}$ & & \\
\hline 284393 & $\mathrm{cbc}$ & 1981-0156 & & $\begin{array}{c}\text { With the Canadian } \\
\text { Force }=[\text { Fighting } \\
\text { Around Grevelles }] \\
\quad:[\text { incomplete }]\end{array}$ & $1917 ?$ & $\mathrm{cn}$ & $\begin{array}{l}\text { Topical Film } \\
\text { Company }\end{array}$ & $\begin{array}{c}\text { War Office } \\
\text { Cinema } \\
\text { Committee for } \\
\text { Canadian War } \\
\text { Records Office }\end{array}$ & & \\
\hline 284394 & $\mathrm{cbc}$ & 1981-0156 & & $\begin{array}{c}\text { British Tommies } \\
\text { in Neuvilly } \\
\text { Offensive }\end{array}$ & N/A & $\mathrm{N} / \mathrm{A}$ & N/A & & & \\
\hline 284395 & $\mathrm{cbc}$ & 1981-0156 & $\begin{array}{c}\text { The } \\
\text { European } \\
\text { War 1914 } \\
\end{array}$ & $\begin{array}{c}\text { The Battle of the } \\
\text { Marne: } \\
\text { [incomplete] }\end{array}$ & N/A & N/A & N/A & & & \\
\hline 284396 & $\mathrm{cbc}$ & 1981-0156 & & $\begin{array}{c}\text { With the Canadian } \\
\text { Force : } \\
\text { [incomplete] }\end{array}$ & 1917 & $\mathrm{uk}$ & $\begin{array}{c}\text { Topical Film } \\
\text { Company }\end{array}$ & $\begin{array}{c}\text { War Office } \\
\text { Cinema } \\
\text { Committee for } \\
\text { Canadian War } \\
\text { Records Office }\end{array}$ & & \\
\hline 284397 & $\mathrm{cbc}$ & 1981-0156 & & $\begin{array}{c}\text { The Regimental } \\
\text { Colours : } \\
\text { [incomplete] }\end{array}$ & 1914/1918? & $\mathrm{fr}$ & Pathe Freres & & OMPB & \\
\hline 284398 & $\mathrm{cbc}$ & 1981-0156 & $\begin{array}{l}\text { The Story of } \\
\text { the Flag }\end{array}$ & $\begin{array}{c}\text { Part One: } \\
\text { [incomplete] }\end{array}$ & N/A & N/A & Anson-Dyer & & & \\
\hline 284399 & $\mathrm{cbc}$ & 1981-0156 & & $\begin{array}{c}\text { [British Troops } \\
\text { Moving Into } \\
\text { Position : } \\
\text { incomplete] }\end{array}$ & N/A & N/A & N/A & & & \\
\hline 284400 & $\mathrm{cbc}$ & 1981-0156 & & $\begin{array}{c}\text { [British Action } \\
\text { Around Lille] } \\
\end{array}$ & 1918 & N/A & N/A & & & \\
\hline 284402 & $\mathrm{cbc}$ & 1981-0156 & $\begin{array}{c}\text { Canadian } \\
\text { Sea Fowl - } \\
\text { Oiseaux de } \\
\text { mer } \\
\text { canadiens } \\
\end{array}$ & $\begin{array}{c}\text { The North Shore - } \\
\text { La Côte Nord: } \\
\text { [incomplete] }\end{array}$ & 1922 & $\mathrm{cn}$ & CGMPB & $\begin{array}{c}\text { National Parks } \\
\text { Bureau, } \\
\text { Department of } \\
\text { Mines and } \\
\text { Resources } \\
\end{array}$ & & \\
\hline 284403 & $\mathrm{cbc}$ & 1981-0156 & & $\begin{array}{l}\text { Life on a French } \\
\text { Training Ship }\end{array}$ & 1915/1924? & $\mathrm{fr}$ & Pathe Freres & & OMPB & \\
\hline 284404 & $\mathrm{cbc}$ & $1981-0156$ & $\begin{array}{c}\text { Canadian } \\
\text { War Records }\end{array}$ & $\begin{array}{l}\text { France's Famous } \\
\text { Gun : The } 75\end{array}$ & N/A & N/A & N/A & & & \\
\hline 284405 & $\mathrm{cbc}$ & 1981-0156 & & $\begin{array}{l}\text { [Infantry : } \\
\text { excerpts] }\end{array}$ & $1914 / 1918$ & $\mathrm{uk}$ & N/A & & & \\
\hline 284407 & $\mathrm{cbc}$ & 1981-0156 & & $\begin{array}{c}\text { Liberation of St. } \\
\text { Quentin and } \\
\text { Ostende } \\
\end{array}$ & N/A & N/A & N/A & & & \\
\hline 284408 & $\mathrm{cbc}$ & 1981-0156 & & $\begin{array}{c}\text { Around } \\
\text { [Labassee] France }\end{array}$ & N/A & N/A & N/A & & & \\
\hline 284409 & $\mathrm{cbc}$ & $1981-0156$ & & $\begin{array}{l}\text { British Troops on } \\
\text { the Western Front }\end{array}$ & N/A & N/A & N/A & & & \\
\hline 284410 & $\mathrm{cbc}$ & 1981-0156 & & $\begin{array}{c}\text { In Action With } \\
\text { Our Canadian } \\
\text { Troops : } \\
\text { [incomplete] = } \\
\text { [With the Royal } \\
\text { Field Artillery In } \\
\text { Action] } \\
\end{array}$ & 1916/1918? & $\mathrm{uk}$ & $\begin{array}{c}\text { Topical Film } \\
\text { Company }\end{array}$ & $\begin{array}{c}\text { War Office } \\
\text { Cinema } \\
\text { Committee for } \\
\text { Canadian War } \\
\text { Records Office } \\
\end{array}$ & & \\
\hline 284411 & $\mathrm{cbc}$ & $1981-0156$ & $\begin{array}{c}\text { Canadian } \\
\text { War Records }\end{array}$ & $\begin{array}{c}\text { Pervyse After the } \\
\text { Battle : } \\
\text { [incomplete] }\end{array}$ & N/A & N/A & N/A & & & \\
\hline 284412 & $\mathrm{cbc}$ & 1981-0156 & $\begin{array}{c}\text { The Invasion } \\
1914\end{array}$ & $\begin{array}{l}\text { After the Retreat } \\
\text { of the German } \\
\text { Army }\end{array}$ & $1914 / 1948$ & $\mathrm{fr}$ & Pathe Freres & & & \\
\hline 284413 & $\mathrm{cbc}$ & 1981-0156 & & $\begin{array}{c}\text { Round Przemysl : } \\
{[\text { excerpt] }}\end{array}$ & 1916 & $\mathrm{fr}$ & Pathe Freres & & & \\
\hline
\end{tabular}




\begin{tabular}{|c|c|c|c|c|c|c|c|c|c|}
\hline 284414 & $\mathrm{cbc}$ & 1981-0156 & $\begin{array}{l}\text { Hepworth Q- } \\
\text { riosity by } \\
\text { "Q" }\end{array}$ & Let's Paint & 1924 & uk & Hepworth & OMPB & \\
\hline 284416 & $\mathrm{cbc}$ & 1981-0156 & & $\begin{array}{c}\text { Convoys of } \\
\text { Provisions in the } \\
\text { Heights of Alsace }\end{array}$ & 1914/1918? & $\mathrm{fr}$ & Pathe Freres & OMPB & \\
\hline 284417 & $\mathrm{cbc}$ & 1981-0156 & & $\begin{array}{l}\text { The African } \\
\text { Zouaves in the } \\
\text { Flanders }\end{array}$ & $1919 ?$ & $\mathrm{fr}$ & Pathe Freres & & \\
\hline 284418 & $\mathrm{cbc}$ & 1981-0156 & $\begin{array}{l}\text { In Action - } \\
\text { The } \\
\text { Canadians }\end{array}$ & $\begin{array}{l}\text { Across the } \\
\text { Battlefields in } \\
\text { France and } \\
\text { Flanders : } \\
\text { incomplete }\end{array}$ & 1917 & $\mathrm{cn}$ & N/A & & \\
\hline 284419 & $\mathrm{cbc}$ & 1981-0156 & $\begin{array}{l}\text { The } \\
\text { European } \\
\text { War }\end{array}$ & $\begin{array}{c}\text { After the German } \\
\text { Retreat } \\
\text { (Continuation) : } \\
\text { [incomplete] }\end{array}$ & $1918 ?$ & $\mathrm{fr}$ & Pathe Freres & OMPB & \\
\hline 284420 & $\mathrm{cbc}$ & 1981-0156 & & $\begin{array}{c}\text { Some German } \\
\text { Cities }\end{array}$ & 1920/1924? & $\mathrm{fr}$ & Pathe Freres & & \\
\hline 284421 & $\mathrm{cbc}$ & 1981-0156 & $\begin{array}{c}\text { Canadian } \\
\text { War Records }\end{array}$ & $\begin{array}{l}\text { [The French Army } \\
\text { : incomplete] }\end{array}$ & N/A & N/A & N/A & & \\
\hline 284422 & $\mathrm{cbc}$ & 1981-0156 & $\begin{array}{c}\text { Army } \\
\text { Review }= \\
\text { Pathé } \\
\text { Journal }\end{array}$ & $\begin{array}{c}\text { [Revue du } 14 \\
\text { juillet 1914] }\end{array}$ & 1914-07-14 ? & N/A & N/A & & \\
\hline 284423 & $\mathrm{cbc}$ & 1981-0156 & & $\begin{array}{l}\text { How the French } \\
\text { Wounded Soldiers } \\
\text { are Looked After } \\
=\text { How French } \\
\text { Wounded Soldiers } \\
\text { are Cared For }\end{array}$ & N/A & $\mathrm{fr}$ & Pathe Freres & & \\
\hline 284424 & $\mathrm{cbc}$ & $1981-0156$ & & Rheims & 1914/1918? & fr & Pathe Freres & & \\
\hline 284426 & $\mathrm{cbc}$ & 1981-0156 & & $\begin{array}{c}\text { Russians } \\
\text { Encamped at } \\
\text { Mailly = Russians } \\
\text { Encamped at } \\
\text { Malay } \\
\end{array}$ & N/A & $\mathrm{fr}$ & Pathe Freres & & \\
\hline 284427 & $\mathrm{cbc}$ & 1981-0156 & & $\begin{array}{l}\text { The Catastrophe } \\
\text { of Avezzano }\end{array}$ & 1920/1924? & $\mathrm{fr}$ & Pathe Freres & & \\
\hline 284428 & $\mathrm{cbc}$ & 1981-0156 & & $\begin{array}{c}\text { Belgium : } \\
\text { [incomplete] }\end{array}$ & N/A & N/A & N/A & & \\
\hline 284429 & $\mathrm{cbc}$ & 1981-0156 & & Field Manoeuvres & N/A & N/A & N/A & & \\
\hline 284430 & $\mathrm{cbc}$ & 1981-0156 & & $\begin{array}{c}\text { Round Przemysl : } \\
\text { [excerpt] }\end{array}$ & 1916 & $\mathrm{fr}$ & Pathe Freres & & \\
\hline 284431 & $\mathrm{cbc}$ & $1981-0156$ & & $\begin{array}{l}\text { France's African } \\
\text { Cavalry on the } \\
\text { Banks of the Yser } \\
: \text { [incomplete] }\end{array}$ & 1914/1918? & $\mathrm{fr}$ & Pathe Freres & & \\
\hline 284433 & $\mathrm{cbc}$ & 1981-0156 & & $\begin{array}{l}\text { Parade of the } \\
\text { Originals : outs }\end{array}$ & 1934 & $\mathrm{cn}$ & $\begin{array}{l}\text { Ontario Motion } \\
\text { Picture Bureau }\end{array}$ & & outtakes \\
\hline 284434 & $\mathrm{cbc}$ & 1981-0156 & & $\begin{array}{l}\text { Felix Tries for } \\
\text { Treasure }\end{array}$ & 1920/1929? & us & N/A & OMPB & \\
\hline 284435 & $\mathrm{cbc}$ & $1981-0156$ & $\begin{array}{l}\text { The Story of } \\
\text { the Flag }\end{array}$ & $\begin{array}{c}\text { Part Five- } \\
\text { [incomplete] }\end{array}$ & N/A & N/A & Anson-Dyer & & \\
\hline 284436 & $\mathrm{cbc}$ & $1981-0156$ & & $\begin{array}{l}\text { The Making of a } \\
\text { King : } \\
\text { [incomplete] }\end{array}$ & $1925 ?$ & us & B.M. Powell Inc. & & \\
\hline 284437 & $\mathrm{cbc}$ & 1981-0156 & $\begin{array}{c}\text { Canadian } \\
\text { War Records }\end{array}$ & $\begin{array}{l}\text { The Indian Army } \\
\text { in France : } \\
\text { [incomplete] }\end{array}$ & 1914/1918? & $\mathrm{fr}$ & Pathe Freres & OMPB & \\
\hline 284438 & $\mathrm{cbc}$ & $1981-0156$ & & $\begin{array}{l}\text { 92nd Highlanders } \\
\text { Battalion Review }\end{array}$ & 1914 & $\mathrm{cn}$ & $\begin{array}{l}\text { Pathescope of } \\
\text { Canada }\end{array}$ & & \\
\hline 284454 & $\mathrm{cbc}$ & 1981-0156 & & $\begin{array}{c}\text { War Dogs : } \\
\text { [incomplete] }\end{array}$ & 1914/1918? & $\mathrm{fr}$ & Pathe Freres & OMPB & \\
\hline 284455 & $\mathrm{cbc}$ & $1981-0156$ & & $\begin{array}{l}\text { Bombardment of } \\
\text { the Bosphorus by } \\
\text { the Russian Fleet : } \\
\text { [incomplete] }\end{array}$ & N/A & N/A & N/A & & \\
\hline
\end{tabular}




\begin{tabular}{|c|c|c|c|c|c|c|c|c|c|c|}
\hline 284456 & $\mathrm{cbc}$ & 1981-0156 & & $\begin{array}{c}\text { The Bank = } \\
\text { Charlot à la } \\
\text { banque = Charlie } \\
\text { at the Bank = } \\
\text { Charlie in the } \\
\text { Bank = Charlie } \\
\text { détective = } \\
\text { Charlot garçon de } \\
\text { banque = Le } \\
\text { Garçon de banque }\end{array}$ & 1915-08-09 & us & $\begin{array}{c}\text { Essanay Film } \\
\text { Manufacturing } \\
\text { Company }\end{array}$ & & $\begin{array}{c}\text { General Film } \\
\text { Company }\end{array}$ & $\begin{array}{l}\text { Tinted } \\
\text { Yellow }\end{array}$ \\
\hline 284457 & $\mathrm{cbc}$ & 1981-0156 & & $\begin{array}{c}\text { General Joffre in } \\
\text { Alsace }\end{array}$ & N/A & N/A & Pathe Freres & & & \\
\hline 284458 & $\mathrm{cbc}$ & 1981-0156 & & $\begin{array}{c}\text { Shanghaied }= \\
\text { Charlot marin = } \\
\text { Charlie the Sailor } \\
\text { = Charlie } \\
\text { Shanghaied }= \\
\text { Charlie on the } \\
\text { Ocean = Charlot } \\
\text { matelot }\end{array}$ & 1915-10-04 & us & $\begin{array}{c}\text { Essanay Film } \\
\text { Manufacturing } \\
\text { Company }\end{array}$ & & & \\
\hline 284459 & $\mathrm{cbc}$ & $1981-0156$ & $\begin{array}{c}\text { Canadian } \\
\text { War Records }\end{array}$ & $\begin{array}{c}\text { [March into } \\
\text { Cologne] : } \\
\text { [incomplete] }\end{array}$ & 1918? & uk & N/A & & OMPB & \\
\hline 284460 & $\mathrm{cbc}$ & 1981-0156 & & $\begin{array}{c}\text { The Gorges of the } \\
\text { Tarn }\end{array}$ & $1921 ?$ & $\mathrm{fr}$ & Pathe Freres & & & \\
\hline 284462 & $\mathrm{cbc}$ & 1981-0156 & & $\begin{array}{l}\text { The British Army. } \\
\text { Saluting the } \\
\text { Colours } \\
\end{array}$ & 1914/1918? & $\mathrm{fr}$ & Pathe Freres & & OMPB & \\
\hline 284463 & $\mathrm{cbc}$ & 1981-0156 & & $\begin{array}{c}\text { State Funeral of } \\
\text { the Late Right } \\
\text { Honourable Sir } \\
\text { Wilfrid Laurier } \\
\text { February 22nd } \\
\end{array}$ & 1919 & $\mathrm{cn}$ & $\begin{array}{l}\text { Pathescope of } \\
\text { Canada }\end{array}$ & & OMPB & \\
\hline 284693 & $\mathrm{cbc}$ & 1981-0156 & & $\begin{array}{c}\text { With the Canadian } \\
\text { Force : } \\
\text { [incomplete] }\end{array}$ & N/A & uk & $\begin{array}{l}\text { Topical Film } \\
\text { Company }\end{array}$ & $\begin{array}{c}\text { War Office } \\
\text { Cinema } \\
\text { Committee for } \\
\text { Canadian War } \\
\text { Records Office } \\
\end{array}$ & & \\
\hline 284700 & $\mathrm{cbc}$ & $1981-0156$ & $\begin{array}{c}\text { The Invasion } \\
\text { of } 1914\end{array}$ & $\begin{array}{l}\text { After the Retreat } \\
\text { of the German } \\
\text { Army : } \\
\text { [incomplete] }\end{array}$ & 1918? & fr & Pathe Freres & & OMPB & \\
\hline 284704 & $\mathrm{cbc}$ & $1981-0156$ & & $\begin{array}{c}\text { [European War }= \\
\text { Official Warning: } \\
\text { incomplete] }\end{array}$ & N/A & N/A & N/A & & & \\
\hline 284706 & $\mathrm{cbc}$ & 1981-0156 & & $\begin{array}{c}\text { [European War }= \\
\text { Official Warning : } \\
\text { incomplete] }\end{array}$ & 1915/1919? & $\mathrm{cn}$ & N/A & & & \\
\hline 293368 & $\mathrm{cbc}$ & 1981-0156 & & $\begin{array}{c}\text { The Canadian Red } \\
\text { Cross : } \\
\text { [incomplete] } \\
\end{array}$ & 1925 & $\mathrm{cn}$ & $\begin{array}{l}\text { Ontario Motion } \\
\text { Picture Bureau }\end{array}$ & & & \\
\hline 440244 & $\mathrm{cbc}$ & 1981-0156 & & $\begin{array}{c}\text { Britain at War }]= \\
\text { European War }= \\
\text { French Artillery }\end{array}$ & $1914 / 1918 ?$ & $\mathrm{fr}$ & Pathe Freres & & & \\
\hline 466409 & $\mathrm{cbc}$ & 1981-0156 & & $\begin{array}{l}\text { Victory Loan } \\
\text { Parade with } \\
\text { British Tank and } \\
\text { American Troops }\end{array}$ & 1918 & N/A & N/A & & & \\
\hline 466489 & $\mathrm{cbc}$ & 1981-0156 & & $\begin{array}{c}\text { London in War } \\
\text { Time and During } \\
\text { Mobilization }\end{array}$ & $1914 / 1918 ?$ & N/A & Pathe Freres & & & \\
\hline 20349 & $\mathrm{cbc}$ & 1981-0156 & & $\begin{array}{c}\text { With the Canadian } \\
\text { Force : } \\
\text { [incomplete] }\end{array}$ & $1916 ?$ & fr & $\begin{array}{l}\text { Topical Film } \\
\text { Company }\end{array}$ & $\begin{array}{c}\text { War Office } \\
\text { Cinema } \\
\text { Committee for } \\
\text { Canadian War } \\
\text { Records Office }\end{array}$ & & \\
\hline 20495 & $\mathrm{cbc}$ & $1981-0156$ & $\begin{array}{c}\text { Canadian } \\
\text { War Records }\end{array}$ & $\begin{array}{c}\text { The Last Post for } \\
\text { Distinguished } \\
\text { Soldier }\end{array}$ & $1929-11$ & $\mathrm{cn}$ & N/A & & & \\
\hline 20498 & $\mathrm{cbc}$ & $1981-0156$ & & $\begin{array}{c}\text { [Cambrai : } \\
\text { incomplete] }\end{array}$ & $1917 ?$ & N/A & N/A & & & \\
\hline 54472 & $\mathrm{cbc}$ & 1981-0156 & $\begin{array}{c}\text { Canadian } \\
\text { War Records }\end{array}$ & $\begin{array}{c}\text { Canadian Hospital } \\
\text { at St. Cloud = }\end{array}$ & 1916 & $\mathrm{cn}$ & $\begin{array}{c}\text { Pathescope of } \\
\text { Canada }\end{array}$ & & OMPB & \\
\hline
\end{tabular}




\begin{tabular}{|c|c|c|c|c|c|c|c|c|c|}
\hline & & & & $\begin{array}{l}\text { Canadian Hospital } \\
\text { at Saint-Cloud }\end{array}$ & & & & & \\
\hline 56766 & $\mathrm{cbc}$ & $1981-0156$ & $\begin{array}{c}\text { Canadian } \\
\text { War Records } \\
\end{array}$ & Ypres and Arras & N/A & $\mathrm{uk}$ & $\begin{array}{l}\text { Topical Film } \\
\text { Company }\end{array}$ & $\begin{array}{c}\text { War Office } \\
\text { Cinema } \\
\text { Committee for } \\
\text { Canadian War } \\
\text { Records Office }\end{array}$ & \\
\hline 56769 & $\mathrm{cbc}$ & 1981-0156 & $\begin{array}{l}\text { In Action - } \\
\text { The } \\
\text { Canadians }\end{array}$ & $\begin{array}{c}\text { Ypres: The Shell- } \\
\text { Shattered City of } \\
\text { Flanders = } \\
\text { Infantry }\end{array}$ & N/A & $\mathrm{uk}$ & $\begin{array}{l}\text { Topical Film } \\
\text { Company }\end{array}$ & $\begin{array}{c}\text { War Office } \\
\text { Cinema } \\
\text { Committee for } \\
\text { Canadian War } \\
\text { Records Office } \\
\end{array}$ & \\
\hline 56774 & $\mathrm{cbc}$ & 1981-0156 & & $\begin{array}{c}\text { [Red Cross Field } \\
\text { Station] : } \\
\text { [incomplete] } \\
\end{array}$ & 1914/1918? & $\mathrm{uk}$ & N/A & & \\
\hline 56776 & $\mathrm{cbc}$ & 1981-0156 & & $\begin{array}{c}\text { Entry into Mons : } \\
\text { [excerpts] }\end{array}$ & 1918 & $\mathrm{cn}$ & N/A & & \\
\hline 90565 & $\mathrm{cbc}$ & 1981-0156 & & $\begin{array}{c}\text { With the Canadian } \\
\text { Force: } \\
\text { [incomplete] }\end{array}$ & 1917 & $\mathrm{uk}$ & $\begin{array}{c}\text { Topical Film } \\
\text { Company }\end{array}$ & $\begin{array}{c}\text { War Office } \\
\text { Cinema } \\
\text { Committee for } \\
\text { Canadian War } \\
\text { Records Office } \\
\end{array}$ & \\
\hline & & \multicolumn{2}{|c|}{$\begin{array}{c}\text { National Film Board of } \\
\text { Canada }\end{array}$} & & & & & & \\
\hline 188766 & NFB & $1982-0218$ & & Assaying Gold & $1920 / 1929$ & $\mathrm{cn}$ & OMPB & & $\begin{array}{c}\text { Tinted } \\
\text { Yellow and } \\
\text { blue }\end{array}$ \\
\hline 379063 & NFB & $1982-0218$ & & A Tale of Talc & 1927 & $\mathrm{cn}$ & OMPB & & $\begin{array}{l}\text { Tinted } \\
\text { Yellow } \\
\end{array}$ \\
\hline & & \multicolumn{2}{|c|}{$\begin{array}{l}\text { National Film Board of } \\
\text { Canada }\end{array}$} & & & & & & \\
\hline 160858 & NFB & 1983-0063 & & $\begin{array}{l}\text { Fishin' Time - } \\
\text { Saison de Peche }\end{array}$ & 1922 & $\mathrm{cn}$ & CGMPB & & $\begin{array}{c}\text { Tinted } \\
\text { unknown } \\
\text { colour. } \\
\text { Bilingual } \\
\text { version, } \\
\text { both french } \\
\text { and english } \\
\text { subtitles } \\
\end{array}$ \\
\hline & & $\begin{array}{c}\text { Ontario } \\
\text { Hydro }\end{array}$ & & & & & & & \\
\hline 104847 & Ontario Hydro & 1983-0249 & & $\begin{array}{l}\text { Tapping Niagara's } \\
\text { Power Resources }\end{array}$ & $1922 ?$ & $\mathrm{cn}$ & $\begin{array}{c}\text { Filmcraft } \\
\text { Industries Ltd. }\end{array}$ & OMPB & $\begin{array}{l}\text { Tinted } \\
\text { unknown } \\
\text { colour } \\
\end{array}$ \\
\hline 171570 & Ontario Hydro & 1983-0249 & & $\begin{array}{c}\text { In the Ontario } \\
\text { Hydro Power } \\
\text { House at } \\
\text { Queenston } \\
\end{array}$ & $1920 ?$ & $\mathrm{cn}$ & $\begin{array}{c}\text { Pathescope of } \\
\text { Canada } \\
\end{array}$ & OMPB & $\begin{array}{l}\text { Tinted } \\
\text { unknown } \\
\text { colour } \\
\end{array}$ \\
\hline 171571 & Ontario Hydro & 1983-0249 & & $\begin{array}{c}\text { Hydro } \\
\text { Development on } \\
\text { the Severn River }= \\
\text { Power } \\
\text { Development on } \\
\text { the Severn River }\end{array}$ & $1922 ?$ & $\mathrm{cn}$ & $\begin{array}{c}\text { Filmcraft } \\
\text { Industries Ltd. }\end{array}$ & $\begin{array}{l}\text { Ontario Hydro- } \\
\text { Electric } \\
\text { Commission } \\
\end{array}$ & \\
\hline 171572 & Ontario Hydro & 1983-0249 & & $\begin{array}{c}\text { Multiplying } \\
\text { Ontario's Power } \\
\text { Resources } \\
\end{array}$ & 1919/1920? & $\mathrm{cn}$ & $\begin{array}{c}\text { Pathescope of } \\
\text { Canada }\end{array}$ & OMPB & $\begin{array}{l}\text { Tinted } \\
\text { unknown } \\
\text { colour } \\
\end{array}$ \\
\hline 302586 & Ontario Hydro & 1983-0249 & & $\begin{array}{c}\text { Queenston- } \\
\text { Chippawa } \\
\text { Development of } \\
\text { the Hydro Electric } \\
\text { Power } \\
\text { Commission of } \\
\text { Ontario } \\
\end{array}$ & 1921 & $\mathrm{cn}$ & $\begin{array}{c}\text { Pathescope of } \\
\text { Canada } \\
\end{array}$ & OMPB & $\begin{array}{l}\text { Tinted } \\
\text { unknown } \\
\text { colour } \\
\end{array}$ \\
\hline 443562 & Ontario Hydro & 1983-0249 & & $\begin{array}{l}\text { Liquid Power: } \\
\text { [incomplete] }\end{array}$ & 1920/1929? & $\mathrm{cn}$ & $\begin{array}{c}\text { Pathescope of } \\
\text { Canada }\end{array}$ & OMPB & $\begin{array}{l}\text { Tinted } \\
\text { unknown } \\
\text { colour } \\
\end{array}$ \\
\hline 41857 & Ontario Hydro & 1983-0249 & & $\begin{array}{l}\text { Installing a Giant } \\
\text { Generator }\end{array}$ & 1920/1922? & $\mathrm{cn}$ & $\begin{array}{l}\text { Pathescope of } \\
\text { Canada }\end{array}$ & OMPB & $\begin{array}{l}\text { Tinted } \\
\text { unknown } \\
\text { colour }\end{array}$ \\
\hline 41859 & Ontario Hydro & 1983-0249 & & $\begin{array}{l}\text { The Chippawa } \\
\text { Canal }\end{array}$ & $1921 ?$ & $\mathrm{cn}$ & $\begin{array}{c}\text { Pathescope of } \\
\text { Canada }\end{array}$ & OMPB & $\begin{array}{l}\text { Tinted } \\
\text { yellow }\end{array}$ \\
\hline
\end{tabular}




\begin{tabular}{|c|c|c|c|c|c|c|c|c|c|}
\hline & & \multicolumn{2}{|c|}{ West Park Hospital Archives } & & & & & & \\
\hline \multirow[t]{2}{*}{59927} & $\begin{array}{l}\text { West Park } \\
\text { Hospital } \\
\text { Archives }\end{array}$ & $1984-0154$ & & $\begin{array}{c}\text { [Sir William } \\
\text { Gage] }\end{array}$ & 1916 & $\mathrm{cn}$ & N/A & & $\begin{array}{c}\text { Tinted } \\
\text { unknow } \\
\text { colour. No } \\
\text { description } \\
\text { or } \\
\text { information } \\
\text { on this film }\end{array}$ \\
\hline & & \multicolumn{2}{|c|}{$\begin{array}{c}\text { Ontario Film } \\
\text { Institute/Ontario Science } \\
\text { Centre } \\
\end{array}$} & & & & & & \\
\hline 5016 & $\begin{array}{c}\text { Ontario Film } \\
\text { Institute/Ontari } \\
\text { o Science } \\
\text { Centre } \\
\end{array}$ & 1985-0310 & & $\begin{array}{c}\text { The Chosen Prince } \\
\text { or The Friendship } \\
\text { of David and } \\
\text { Jonathan }\end{array}$ & 1917 & us & $\begin{array}{l}\text { The Crest } \\
\text { Picture } \\
\text { Company }\end{array}$ & & \\
\hline 5018 & $\begin{array}{l}\text { Ontario Film } \\
\text { Institute/Ontari } \\
\text { o Science } \\
\text { Centre }\end{array}$ & $1985-0310$ & & $\begin{array}{l}\text { The Story of a } \\
\text { Cocoa Bean : } \\
\text { [incomplete] }\end{array}$ & $1918-1925 ?$ & $\mathrm{cn}$ & $\begin{array}{l}\text { Pathescope of } \\
\text { Canada }\end{array}$ & & \\
\hline 5019 & $\begin{array}{c}\text { Ontario Film } \\
\text { Institute/Ontari } \\
\text { o Science } \\
\text { Centre } \\
\end{array}$ & 1985-0310 & & $\begin{array}{l}\text { The Wishing Ring } \\
: \text { [incomplete] }\end{array}$ & 1914 & us & $\begin{array}{l}\text { World Film } \\
\text { Corporation } \\
\end{array}$ & & $\begin{array}{c}\text { Tinted pink } \\
\text { and blue. } \\
\text { Directed by } \\
\text { Maurice } \\
\text { Tourneur } \\
\end{array}$ \\
\hline 5020 & $\begin{array}{c}\text { Ontario Film } \\
\text { Institute/Ontari } \\
\text { o Science } \\
\text { Centre } \\
\end{array}$ & 1985-0310 & & Alaska & $1931 ?$ & $\mathrm{cn}$ & $\begin{array}{l}\text { Canadian } \\
\text { National } \\
\text { Railway } \\
\end{array}$ & & \\
\hline 5021 & $\begin{array}{c}\text { Ontario Film } \\
\text { Institute/Ontari } \\
\text { o Science } \\
\text { Centre }\end{array}$ & $1985-0310$ & & $\begin{array}{c}\text { Ontario Summer } \\
\text { Sorceries }\end{array}$ & N/A & $\mathrm{cn}$ & N/A & & \\
\hline 5022 & $\begin{array}{c}\text { Ontario Film } \\
\text { Institute/Ontari } \\
\text { o Science } \\
\text { Centre } \\
\end{array}$ & 1985-0310 & & $\begin{array}{c}\text { [Unidentified } \\
\text { Drama : excerpt] }\end{array}$ & N/A & N/A & N/A & & $\begin{array}{c}\text { No } \\
\text { information } \\
\text { this title }\end{array}$ \\
\hline 5024 & $\begin{array}{c}\text { Ontario Film } \\
\text { Institute/Ontari } \\
\text { o Science } \\
\text { Centre } \\
\end{array}$ & $1985-0310$ & & $\begin{array}{c}\text { [Unidentified } \\
\text { Pirate Drama : } \\
\text { excerpt] }\end{array}$ & N/A & $\mathrm{N} / \mathrm{A}$ & $\mathrm{N} / \mathrm{A}$ & & $\begin{array}{c}\text { Tinted red } \\
\text { and blue. No } \\
\text { information } \\
\text { on this title } \\
\end{array}$ \\
\hline 5025 & $\begin{array}{c}\text { Ontario Film } \\
\text { Institute/Ontari } \\
\text { o Science } \\
\text { Centre }\end{array}$ & $1985-0310$ & & $\begin{array}{l}\text { Saskatchewan } \\
\text { Schools and the } \\
\text { New Canadians }\end{array}$ & 1916/1922? & $\mathrm{cn}$ & N/A & $\begin{array}{l}\text { Saskatchewan } \\
\text { Schools and the } \\
\text { New Canadians }\end{array}$ & $\begin{array}{l}\text { Tinted } \\
\text { yellow and } \\
\text { orange }\end{array}$ \\
\hline 5026 & $\begin{array}{c}\text { Ontario Film } \\
\text { Institute/Ontari } \\
\text { o Science } \\
\text { Centre } \\
\end{array}$ & $1985-0310$ & & $\begin{array}{c}\text { [Empress of Asia : } \\
\text { incomplete] }\end{array}$ & 1920 & $\mathrm{cn}$ & $\begin{array}{c}\text { Pathescope of } \\
\text { Canada }\end{array}$ & $\begin{array}{c}\text { Canadian Pacific } \\
\text { Railway } \\
\text { Company } \\
\end{array}$ & \\
\hline 5027 & $\begin{array}{c}\text { Ontario Film } \\
\text { Institute/Ontari } \\
\text { o Science } \\
\text { Centre } \\
\end{array}$ & 1985-0310 & & $\begin{array}{l}\text { 92nd Highlanders } \\
\text { Battalion Review }\end{array}$ & 1914 & $\mathrm{cn}$ & N/A & & \\
\hline \multirow[t]{2}{*}{5023} & $\begin{array}{c}\text { Ontario Film } \\
\text { Institute/Ontari } \\
\text { o Science } \\
\text { Centre } \\
\end{array}$ & $1985-0310$ & $\begin{array}{l}\text { Seeing } \\
\text { Canada }\end{array}$ & $\begin{array}{l}\text { The Playground of } \\
\text { a Continent : } \\
\text { [excerpt] }\end{array}$ & 1919 & $\mathrm{cn}$ & $\begin{array}{c}\text { Exhibits and } \\
\text { Publicity Bureau }\end{array}$ & $\begin{array}{l}\text { Department of } \\
\text { Trade and } \\
\text { Commerce } \\
\end{array}$ & $\begin{array}{l}\text { Tinted } \\
\text { yellow }\end{array}$ \\
\hline & & $\begin{array}{c}\text { INCO } \\
\text { Limited } \\
\end{array}$ & & & & & & & \\
\hline \multirow[t]{2}{*}{61250} & INCO Limited & 1986-0384 & & $\begin{array}{l}\text { The Mining and } \\
\text { Smelting of } \\
\text { Canadian Nickel }\end{array}$ & $1918 ?$ & $\mathrm{cn}$ & $\begin{array}{c}\text { Pathescope of } \\
\text { Canada }\end{array}$ & $\begin{array}{c}\text { The Mond } \\
\text { Nickel Company } \\
\text { Ltd } \\
\end{array}$ & $\begin{array}{l}\text { Tinted } \\
\text { yellow }\end{array}$ \\
\hline & & $\begin{array}{l}\text { Kilfoyle, } \\
\text { William }\end{array}$ & & & & & & & \\
\hline
\end{tabular}




\begin{tabular}{|c|c|c|c|c|c|c|c|c|c|c|}
\hline 28862 & $\begin{array}{l}\text { Kilfoyle, } \\
\text { William }\end{array}$ & 1986-0549 & & $\begin{array}{l}\text { The Kemptville } \\
\text { Agricultural } \\
\text { School: [excerpt] }\end{array}$ & $1923 / 1929$ & $\mathrm{cn}$ & $\begin{array}{l}\text { Ontario Motion } \\
\text { Picture Bureau }\end{array}$ & & & \\
\hline 28863 & $\begin{array}{l}\text { Kilfoyle, } \\
\text { William }\end{array}$ & 1986-0549 & & $\begin{array}{c}\text { [Bobby Bumps on } \\
\text { the Doughnut } \\
\text { Trail] } \\
\end{array}$ & 1918 & us & $\begin{array}{l}\text { J.R. Bray } \\
\text { Studios Inc } \\
\end{array}$ & & $\begin{array}{l}\text { Paramount } \\
\text { Pictures } \\
\end{array}$ & $\begin{array}{l}\text { Tinted } \\
\text { unknow } \\
\text { colour } \\
\end{array}$ \\
\hline 28864 & $\begin{array}{l}\text { Kilfoyle, } \\
\text { William }\end{array}$ & 1986-0549 & & $\begin{array}{l}\text { Making the Most } \\
\text { of Manure }\end{array}$ & $1917 / 1923$ & $\mathrm{cn}$ & OMPB & & & \\
\hline & & \multicolumn{2}{|c|}{$\begin{array}{c}\text { Canadian Broadcasting } \\
\text { Corporation }\end{array}$} & & & & & & & \\
\hline 235796 & $\mathrm{cbc}$ & 1986-0810 & & $\begin{array}{c}\text { [Matthews' } \\
\text { Archives] From } \\
\text { Brown Betty to } \\
\text { Court Lane } \\
\text { September } 1910 \\
\end{array}$ & 1935/1940? & $\mathrm{cn}$ & $\begin{array}{c}\text { Chuck Matthews } \\
\text { (producer) }\end{array}$ & & & $\begin{array}{c}\text { Recreation } \\
\text { of move of } \\
\text { Toronto } \\
\text { Arts and } \\
\text { Letters } \\
\text { Club. } \\
\text { Amateur } \\
\text { film? }\end{array}$ \\
\hline & & $\begin{array}{l}\text { Crawley } \\
\text { Films } \\
\text { Limited } \\
\end{array}$ & & & & & & & & \\
\hline \multirow[t]{2}{*}{60397} & $\begin{array}{l}\text { Crawley Films } \\
\text { Limited }\end{array}$ & $1987-0423$ & & $\begin{array}{c}\text { Queen's } \\
\text { University } 1841- \\
1926: 85 \text { Years of } \\
\text { Loyal Service }\end{array}$ & 1926 & $\mathrm{cn}$ & $\begin{array}{l}\text { Ontario Motion } \\
\text { Picture Bureau }\end{array}$ & $\begin{array}{c}\text { Queen's } \\
\text { University }\end{array}$ & & $\begin{array}{l}\text { Tinted } \\
\text { Yellow } \\
\end{array}$ \\
\hline & & $\begin{array}{c}\text { General } \\
\text { Sound }\end{array}$ & & & & & & & & \\
\hline 87799 & General Sound & 1988-0045 & & $\begin{array}{c}\text { [Eminent Senior } \\
\text { Golfers Play at } \\
\text { Scarboro] }\end{array}$ & N/A & $\mathrm{cn}$ & N/A & & & $\begin{array}{c}\text { No } \\
\text { information } \\
\text { on this title }\end{array}$ \\
\hline 87800 & General Sound & 1988-0045 & & $\begin{array}{c}\text { [The Prince of } \\
\text { Wales in Canada : } \\
\text { incomplete] }\end{array}$ & N/A & $\mathrm{cn}$ & $\begin{array}{c}\text { Pathescope of } \\
\text { Canada }\end{array}$ & & & \\
\hline 87801 & General Sound & 1988-0045 & & $\begin{array}{c}\text { [Prince of Wales } \\
\text { in Canada : } \\
\text { incomplete] } \\
\end{array}$ & 1919 & $\mathrm{cn}$ & $\begin{array}{c}\text { Pathescope of } \\
\text { Canada }\end{array}$ & & & \\
\hline & & \multicolumn{2}{|c|}{$\begin{array}{l}\text { British Columbia Archives } \\
\text { and Records Service }\end{array}$} & & & & & & & \\
\hline 87934 & $\begin{array}{c}\text { British } \\
\text { Columbia } \\
\text { Archives and } \\
\text { Records } \\
\text { Service } \\
\end{array}$ & 1988-0239 & & $\begin{array}{l}\text { A Victory Loan } \\
\text { Appeal : [public } \\
\text { service } \\
\text { announcement] }\end{array}$ & 1918 & $\mathrm{cn}$ & Famous Players & $\begin{array}{c}\text { National } \\
\text { Association of } \\
\text { the Motion } \\
\text { Picture Industry; } \\
\text { Dominion } \\
\text { Motion Picture } \\
\text { Distribution } \\
\text { Committee; } \\
\text { Dominion } \\
\text { Publicity } \\
\text { Committee } \\
\end{array}$ & & \\
\hline & & $\begin{array}{c}\text { Archives of } \\
\text { Ontario }\end{array}$ & & & & & & & & \\
\hline 224891 & $\begin{array}{c}\text { Archives of } \\
\text { Ontario }\end{array}$ & 1994-0166 & & $\begin{array}{l}\text { Summer Training } \\
\text { for Schoolboys }\end{array}$ & $1924 / 1930$ & $\mathrm{cn}$ & $\begin{array}{l}\text { Ontario Motion } \\
\text { Picture Bureau }\end{array}$ & $\begin{array}{c}\text { Ontario Athletic } \\
\text { Commission }\end{array}$ & & \\
\hline 224892 & $\begin{array}{c}\text { Archives of } \\
\text { Ontario }\end{array}$ & 1994-0166 & & $\begin{array}{l}\text { Hired Out : } \\
\text { [incomplete] }\end{array}$ & $1922 ?$ & $\mathrm{cn}$ & $\begin{array}{l}\text { Ontario Motion } \\
\text { Picture Bureau } \\
\end{array}$ & & & $\begin{array}{l}\text { Tinted } \\
\text { unknown } \\
\text { colour } \\
\end{array}$ \\
\hline 224975 & $\begin{array}{c}\text { Archives of } \\
\text { Ontario } \\
\end{array}$ & 1994-0166 & & $\begin{array}{c}\text { Manufacture of } \\
\text { Bamboo Hats }\end{array}$ & $1920 / 1922$ & $\mathrm{~N} / \mathrm{A}$ & Pathe Freres & & & $\begin{array}{l}\text { Tinted } \\
\text { unknown } \\
\text { colour } \\
\end{array}$ \\
\hline 224976 & $\begin{array}{c}\text { Archives of } \\
\text { Ontario }\end{array}$ & 1994-0166 & & The Stonghold & N/A & N/A & N/A & & & $\begin{array}{c}\text { Tinted } \\
\text { orange. No } \\
\text { information } \\
\text { on this title } \\
\end{array}$ \\
\hline 224977 & $\begin{array}{c}\text { Archives of } \\
\text { Ontario }\end{array}$ & 1994-0166 & & $\begin{array}{l}\text { The Torpedo : } \\
\text { Hornet of the Sea }\end{array}$ & 1917 & us & $\begin{array}{c}\text { Bray } \\
\text { Productions Inc. }\end{array}$ & & $\begin{array}{l}\text { Paramount- } \\
\text { Bray- } \\
\text { Pictographs }\end{array}$ & \\
\hline 224978 & $\begin{array}{c}\text { Archives of } \\
\text { Ontario }\end{array}$ & 1994-0166 & & $\begin{array}{c}\text { The Trail of the } \\
\text { Gods : } \\
\text { [incomplete] }\end{array}$ & 1920/1929? & N/A & Noel Gordon & & & $\begin{array}{c}\text { No } \\
\text { information } \\
\end{array}$ \\
\hline 224979 & $\begin{array}{c}\text { Archives of } \\
\text { Ontario }\end{array}$ & 1994-0166 & & $\begin{array}{l}\text { Where Does False } \\
\text { Hair Come From? }\end{array}$ & N/A & N/A & N/A & & & \\
\hline 224980 & $\begin{array}{c}\text { Archives of } \\
\text { Ontario }\end{array}$ & 1994-0166 & & $\begin{array}{c}\text { The Unlucky } \\
\text { Portrait }\end{array}$ & $\mathrm{N} / \mathrm{A}$ & $\mathrm{N} / \mathrm{A}$ & $\mathrm{N} / \mathrm{A}$ & & & \\
\hline
\end{tabular}




\begin{tabular}{|c|c|c|c|c|c|c|c|c|c|}
\hline 226702 & $\begin{array}{l}\text { Archives of } \\
\text { Ontario }\end{array}$ & 1994-0166 & & $\begin{array}{c}\text { Summer Training } \\
\text { for Schoolboys : } \\
\text { [out-takes] }\end{array}$ & N/A & $\mathrm{cn}$ & $\begin{array}{l}\text { Ontario Motion } \\
\text { Picture Bureau }\end{array}$ & $\begin{array}{l}\text { Ontario Athletic } \\
\text { Commission }\end{array}$ & outtakes \\
\hline 226704 & $\begin{array}{l}\text { Archives of } \\
\text { Ontario }\end{array}$ & 1994-0166 & $\begin{array}{c}\text { Ontario } \\
\text { Athletic } \\
\text { Commission } \\
\text { Athletic } \\
\text { Film } \\
\end{array}$ & $\begin{array}{c}\text { [Wrestling and } \\
\text { Handball in Slow } \\
\text { Motion] }\end{array}$ & N/A & $\mathrm{cn}$ & $\begin{array}{c}\text { Ontario Motion } \\
\text { Picture Bureau } \\
\end{array}$ & $\begin{array}{l}\text { Ontario Athletic } \\
\text { Commission }\end{array}$ & $\begin{array}{c}\text { Also } \\
\text { produced by } \\
\text { Novagraph } \\
\text { Film } \\
\text { Corporation } \\
\end{array}$ \\
\hline 226705 & $\begin{array}{l}\text { Archives of } \\
\text { Ontario } \\
\end{array}$ & 1994-0166 & $\begin{array}{c}\text { Ontario } \\
\text { Athletic } \\
\text { Commission } \\
\text { Athletic } \\
\text { Film } \\
\end{array}$ & $\begin{array}{c}{[\text { Famous Ball }} \\
\text { Players in Action] }\end{array}$ & 1917 & $\mathrm{cn}$ & $\begin{array}{c}\text { Ontario Motion } \\
\text { Picture Bureau }\end{array}$ & $\begin{array}{l}\text { Ontario Athletic } \\
\text { Commission }\end{array}$ & $\begin{array}{l}\text { Tinted } \\
\text { yellow }\end{array}$ \\
\hline 226707 & $\begin{array}{l}\text { Archives of } \\
\text { Ontario } \\
\end{array}$ & 1994-0166 & $\begin{array}{c}\text { Ontario } \\
\text { Athletic } \\
\text { Commission } \\
\text { Athletic } \\
\text { Film } \\
\end{array}$ & $\begin{array}{l}\text { [Track and Field } \\
\text { in Slow Motion] }\end{array}$ & N/A & $\mathrm{cn}$ & $\begin{array}{c}\text { Ontario Motion } \\
\text { Picture Bureau } \\
\end{array}$ & $\begin{array}{l}\text { Ontario Athletic } \\
\text { Commission }\end{array}$ & \\
\hline 226708 & $\begin{array}{l}\text { Archives of } \\
\text { Ontario } \\
\end{array}$ & 1994-0166 & $\begin{array}{c}\text { Ontario } \\
\text { Athletic } \\
\text { Commission } \\
\text { Athletic } \\
\text { Film } \\
\end{array}$ & $\begin{array}{l}\text { [Inside Facts on } \\
\text { Field Sports] }\end{array}$ & N/A & $\mathrm{cn}$ & $\begin{array}{c}\text { Ontario Motion } \\
\text { Picture Bureau }\end{array}$ & $\begin{array}{l}\text { Ontario Athletic } \\
\text { Commission }\end{array}$ & $\begin{array}{l}\text { Tinted } \\
\text { yellow }\end{array}$ \\
\hline 226711 & $\begin{array}{c}\text { Archives of } \\
\text { Ontario } \\
\end{array}$ & 1994-0166 & $\begin{array}{c}\text { Ontario } \\
\text { Athletic } \\
\text { Commission } \\
\text { Athletic } \\
\text { Film } \\
\end{array}$ & $\begin{array}{c}\text { [Sports } \\
\text { Movements in } \\
\text { Slow Motion] } \\
\end{array}$ & N/A & $\mathrm{cn}$ & $\begin{array}{c}\text { Ontario Motion } \\
\text { Picture Bureau }\end{array}$ & $\begin{array}{l}\text { Ontario Athletic } \\
\text { Commission }\end{array}$ & \\
\hline & & \multicolumn{2}{|c|}{ Northeast Historic Film } & & & & & & \\
\hline 290386 & $\begin{array}{c}\text { Northeast } \\
\text { Historic Film }\end{array}$ & 1999-0009 & $\begin{array}{l}\text { Seeing } \\
\text { Canada }\end{array}$ & Unblazed Trails & 1920 & $\mathrm{cn}$ & $\begin{array}{c}\text { Exhibits and } \\
\text { Publicity Bureau }\end{array}$ & & $\begin{array}{l}\text { Tinted aqua } \\
\text { and orange }\end{array}$ \\
\hline & & \multicolumn{2}{|c|}{$\begin{array}{c}\text { Canadian Broadcasting } \\
\text { Corporation }\end{array}$} & & & & & & \\
\hline 302951 & $\mathrm{cbc}$ & 1999-0036 & & $\begin{array}{c}\text { The Prince of } \\
\text { Wales in Canada : } \\
\text { [incomplete] }\end{array}$ & 1919/1920? & $\mathrm{cn}$ & $\begin{array}{l}\text { Pathescope of } \\
\text { Canada }\end{array}$ & & \\
\hline 302952 & $\mathrm{cbc}$ & 1999-0036 & & $\begin{array}{l}\text { A Victory Loan } \\
\text { Appeal : [public } \\
\text { service } \\
\text { announcement] }\end{array}$ & 1918 & $\mathrm{cn}$ & Famous Players & $\begin{array}{c}\text { National } \\
\text { Association of } \\
\text { the Motion } \\
\text { Picture Industry; } \\
\text { Dominion } \\
\text { Motion Picture } \\
\text { Distribution } \\
\text { Committee; } \\
\text { Dominion } \\
\text { Publicity } \\
\text { Committee } \\
\end{array}$ & $\begin{array}{l}\text { Produced in } \\
\text { both US and } \\
\text { Canada and } \\
\text { this is the } \\
\text { Canadian } \\
\text { version }\end{array}$ \\
\hline 302953 & $\mathrm{cbc}$ & 1999-0036 & & Prosperity & $1919 ?$ & $\mathrm{cn}$ & $\begin{array}{l}\text { Pathescope of } \\
\text { Canada }\end{array}$ & $\begin{array}{c}\text { Dominion } \\
\text { Publicity } \\
\text { Committee } \\
\end{array}$ & \\
\hline & & Lyon, John & & & & & & & \\
\hline 303545 & Lyon, John & 1999-0040 & & $\begin{array}{l}\text { Scarborough War } \\
\text { Memorial } \\
\text { Unveiled by Earl } \\
\text { Jellicoe } \\
\end{array}$ & $1931 ?$ & $\mathrm{cn}$ & N/A & & \\
\hline 303546 & Lyon, John & 1999-0040 & & $\begin{array}{c}\text { Canadian Soldiers } \\
\text { in the Making: } \\
\text { [incomplete] }\end{array}$ & 1915 & $\mathrm{cn}$ & $\begin{array}{l}\text { Pathescope of } \\
\text { Canada } \\
\end{array}$ & & \\
\hline 303547 & Lyon, John & 1999-0040 & & $\begin{array}{c}\text { Technical } \\
\text { Education : } \\
\text { [incomplete] }\end{array}$ & 1931/1933? & $\mathrm{cn}$ & $\begin{array}{l}\text { Ontario Motion } \\
\text { Picture Bureau }\end{array}$ & & $\begin{array}{l}\text { Shot at } \\
\text { Central } \\
\text { Tech in } \\
\text { Toronto }\end{array}$ \\
\hline 303548 & Lyon, John & $1999-0040$ & & $\begin{array}{c}\text { Ontario's Summer } \\
\text { Sorceries : } \\
\text { [incomplete] } \\
\end{array}$ & 1923/1929? & $\mathrm{cn}$ & $\begin{array}{c}\text { Ontario Motion } \\
\text { Picture Bureau } \\
\end{array}$ & & \\
\hline 303549 & Lyon, John & 1999-0040 & & $\begin{array}{c}\text { [British Empire } \\
\text { Games : Hamilton } \\
: \text { incomplete] }\end{array}$ & $1930 ?$ & $\mathrm{cn}$ & $\begin{array}{l}\text { Ontario Motion } \\
\text { Picture Bureau }\end{array}$ & & \\
\hline 303550 & Lyon, John & 1999-0040 & & $\begin{array}{c}\text { Speeding Up Your } \\
\text { Business : } \\
\text { [incomplete] }\end{array}$ & 1924/1929? & $\mathrm{cn}$ & $\begin{array}{l}\text { Ontario Motion } \\
\text { Picture Bureau }\end{array}$ & & \\
\hline
\end{tabular}




\begin{tabular}{|c|c|c|c|c|c|c|c|c|c|c|}
\hline 303551 & Lyon, John & 1999-0040 & & $\begin{array}{c}\text { Ontario School for } \\
\text { the Deaf : } \\
\text { [incomplete }\end{array}$ & 1925 & $\mathrm{cn}$ & $\begin{array}{l}\text { Ontario Motion } \\
\text { Picture Bureau }\end{array}$ & & & $\begin{array}{l}\text { Tinted } \\
\text { yellow }\end{array}$ \\
\hline 303552 & Lyon, John & 1999-0040 & & $\begin{array}{c}\text { More Power for } \\
\text { the Twin Cities: } \\
\text { [incomplete] }\end{array}$ & $1922 ?$ & $\mathrm{cn}$ & $\begin{array}{c}\text { Pathescope of } \\
\text { Canada } \\
\end{array}$ & OMPB & & $\begin{array}{l}\text { Tinted } \\
\text { yellow }\end{array}$ \\
\hline 303553 & Lyon, John & 1999-0040 & & $\begin{array}{c}\text { [Smelting Ore: } \\
\text { incomplete] }\end{array}$ & 1923/1930? & $\mathrm{cn}$ & N/A & & & \\
\hline 303554 & Lyon, John & 1999-0040 & & $\begin{array}{c}\text { Prospecting for } \\
\text { Gold : } \\
\text { [incomplete] }\end{array}$ & 1921/1922? & $\mathrm{cn}$ & $\begin{array}{c}\text { Filmcraft } \\
\text { Industries Ltd. }\end{array}$ & OMPB & & $\begin{array}{l}\text { Tinted } \\
\text { yellow }\end{array}$ \\
\hline 303555 & Lyon, John & 1999-0040 & & $\begin{array}{c}\text { Canada Welcomes } \\
\text { Kaye Don : } \\
\text { Citizens of } \\
\text { Toronto Give } \\
\text { Great Ovation to } \\
\text { Empire Hero } \\
\end{array}$ & 1931 & $\mathrm{cn}$ & $\begin{array}{c}\text { Ontario Motion } \\
\text { Picture Bureau }\end{array}$ & & & \\
\hline 303556 & Lyon, John & 1999-0040 & & $\begin{array}{c}\text { The Victory Fêtes } \\
: \text { [incomplete }]\end{array}$ & $1919 ?$ & N/A & Pathe Freres & & & \\
\hline 303557 & Lyon, John & $1999-0040$ & & [Zoo Animals] & 1920/1930? & N/A & N/A & & & \\
\hline \multirow[t]{2}{*}{307506} & Lyon, John & 1999-0040 & & $\begin{array}{c}\text { Resuscitation : } \\
\text { [incomplete] }\end{array}$ & $1917 / 1922$ & $\mathrm{cn}$ & $\begin{array}{l}\text { Ontario Motion } \\
\text { Picture Bureau } \\
\end{array}$ & & & \\
\hline & & Lyon, John & & & & & & & & \\
\hline 309297 & Lyon, John & $2000-0018$ & & $\begin{array}{c}\text { After } 100 \text { Years : } \\
\text { [incomplete] }\end{array}$ & 1927 & $\mathrm{cn}$ & OMPB & & & $\begin{array}{l}\text { Film at } \\
\text { UofT }\end{array}$ \\
\hline 309298 & Lyon, John & $2000-0018$ & & $\begin{array}{c}\text { Transport in the } \\
\text { North: } \\
\text { [incomplete] }\end{array}$ & 1925 & $\mathrm{cn}$ & OMPB & & & \\
\hline 309299 & Lyon, John & 2000-0018 & & $\begin{array}{c}\text { [Men's Swim } \\
\text { C.N.E. - 1931 : } \\
\text { incomplete] } \\
\end{array}$ & $1931 ?$ & cn & OMPB & & & \\
\hline 309300 & Lyon, John & $2000-0018$ & $\begin{array}{c}\text { Oriental } \\
\text { Cruise Series } \\
\end{array}$ & $\begin{array}{c}\text { In the Realm of } \\
\text { the White Rajah: } \\
\text { [incomplete] }\end{array}$ & 1928 & $\mathrm{cn}$ & OMPB & & & $\begin{array}{c}\text { photography } \\
\text {, George } \\
\text { Rutherford }\end{array}$ \\
\hline & & $\begin{array}{c}\text { Breck, } \\
\text { William } \\
\end{array}$ & & & & & & & & \\
\hline 319603 & Breck, William & $2000-0057$ & & $\begin{array}{c}\text { [Throop Family : } \\
\text { home movie] }\end{array}$ & $1924 ?$ & $\mathrm{cn}$ & N/A & & & $\begin{array}{c}\text { Amateur } \\
\text { film maybe } \\
\text { shot by } \\
\text { George E. } \\
\text { Patton }\end{array}$ \\
\hline & & King, Bruce & & & & & & & & \\
\hline 387663 & King, Bruce & 2008-0021 & & $\begin{array}{l}\text { Camouflage: } \\
\text { What It Is and } \\
\text { What It Does }\end{array}$ & 1917 & us & $\begin{array}{c}\text { Daypho-Bray } \\
\text { Productions Inc. }\end{array}$ & & OMPB & $\begin{array}{l}\text { Tinted } \\
\text { unknown } \\
\text { colour }\end{array}$ \\
\hline 387639 & King, Bruce & 2008-0021 & & $\begin{array}{c}\text { Ontario VCs leave } \\
\text { for London }\end{array}$ & 1919 & $\mathrm{cn}$ & N/A & & & \\
\hline 387647 & King, Bruce & $2008-0021$ & $\begin{array}{l}\text { Canadian } \\
\text { National } \\
\text { Pictorial } \\
\end{array}$ & $\begin{array}{l}\text { The Indian Army } \\
\text { in France }\end{array}$ & 1914/1918? & fr & Pathe Freres & & OMPB & \\
\hline 388339 & King, Bruce & 2008-0021 & & $\begin{array}{c}\text { Mobilization of } \\
\text { the French Fleet : } \\
\text { [incomplete] }\end{array}$ & 1914/1918? & $\mathrm{fr}$ & Pathe Freres & & OMPB & \\
\hline & & King, Bruce & & & & & & & & \\
\hline 388066 & King, Bruce & 2008-0022 & & $\begin{array}{c}\text { [French Army } \\
\text { Gymnastics] }\end{array}$ & 1914/1918? & $\mathrm{fr}$ & Pathe Freres & & OMPB & \\
\hline 388291 & King, Bruce & $2008-0022$ & & $\begin{array}{l}\text { The French Army } \\
\text { on the March }\end{array}$ & 1914/1918? & $\mathrm{fr}$ & Pathe Freres & & OMPB & \\
\hline 388295 & King, Bruce & $2008-0022$ & & $\begin{array}{l}\text { Bombardment of } \\
\text { the Bosphorus by } \\
\text { the Russian Fleet }\end{array}$ & & N/A & Pathe Freres & & OMPB & \\
\hline 388306 & King, Bruce & 2008-0022 & & $\begin{array}{c}\text { British Empire } \\
\text { Games } \\
\end{array}$ & 1930 & $\mathrm{cn}$ & OMPB & & & \\
\hline
\end{tabular}




\begin{tabular}{|c|c|c|c|c|c|c|c|}
\hline & & $\begin{array}{c}\text { Patton, } \\
\text { George E. }\end{array}$ & & & & & \\
\hline 388879 & $\begin{array}{c}\text { Patton, George } \\
\text { E. }\end{array}$ & 2008-0046 & $\begin{array}{c}\text { [George Patton } \\
\text { No. } 1 \text { = Wedding : } \\
\text { home movie] }\end{array}$ & $1920 / 1925 ?$ & $\mathrm{cn}$ & N/A & $\begin{array}{c}\text { George } \\
\text { Patton, } \\
\text { director of } \\
\text { OMPB }\end{array}$ \\
\hline 388880 & $\begin{array}{c}\text { Patton, George } \\
\text { E. }\end{array}$ & 2008-0046 & $\begin{array}{c}\text { [George Patton } \\
\text { No. } 2=\text { At Home } \\
\text { With the Pattons : } \\
\text { home movie] }\end{array}$ & 1925/1930? & $\mathrm{cn}$ & N/A & $\begin{array}{c}\text { George } \\
\text { Patton, } \\
\text { director of } \\
\text { OMPB }\end{array}$ \\
\hline 388881 & $\begin{array}{l}\text { Patton, George } \\
\text { E. }\end{array}$ & 2008-0046 & $\begin{array}{c}\text { [George Patton } \\
\text { No. } 3= \\
\text { Swimming : home } \\
\text { movie] }\end{array}$ & 1930/1934? & $\mathrm{cn}$ & N/A & $\begin{array}{c}\text { George } \\
\text { Patton, } \\
\text { director of } \\
\text { OMPB }\end{array}$ \\
\hline
\end{tabular}




\section{Bibliography}

Backhouse, Charles F. Canadian Government Motion Picture Bureau 1917-1941. Ottawa: Canadian Film Institute, 1974.

Bergeron, Rosemary. "A History of the Newsreel in Canada: A Struggle for Screen Time." The Movie Image 7, no. 2 (Fall 2007): 25-54. https://muse-jhuedu.ezproxy.lib.ryerson.ca/article/235600/pdf

Brown, Harold. Physical Characteristics of Early Films as Aids to Identification. Brussels: FIAF, 1990.

Cherchi Usai, Paolo. Silent Cinema: An Introduction. London: BFI Publishing, 2000

Cook, Sarah. "Shrouded History: The Canadian Film and Photo Unit, Records Creation, Reuse, and the Recontextualizing of "Lost" Audiovisual Heritage." Archivaria 83, (Spring 2017): 125148.

Constantinides, Zoë. "The Myth of Evangeline and the Origin of Canadian National Cinema." Film History 26, no. 1 (2014): 50-74. https://muse-jhuedu.ezproxy.lib.ryerson.ca/article/542047/pdf

Crawford, Merritt. "Movie Makers The first thirty years." Movie Makers (December 1930): 755785. https://muse-jhu-edu.ezproxy.lib.ryerson.ca/article/235600/pdf

Evans, Gary. In the national interest: a chronicle of the National Film Board of Canada from 1949 to 1989. University of Toronto Press, 1991.

Flueckiger, Barbara. "Edge Codes and Identification - Timeline of Historical Film colors." Accessed August 11, 2019. https://filmcolors.org/timeline-entry/16668/\#/

Gorman, Michael, ed. The Concise AACR2 Fourth edition, Ottawa: Canadian Library Association, 2004. https://epdf.pub/the-concise-aacr2.html

Graham, Gerald. Canadian Film Technology, 1896 - 1986. London: Associated University Press, 1989.

Mitchell, J.R. "The Beacon Portable Motion Picture Projector." Transactions of the Society of Motion Picture Engineers 16, (May 1923): 225-232. https://muse-jhuedu.ezproxy.lib.ryerson.ca/article/235600/pdf 
Morris, Peter. Embattled Shadows: A History of Canadian Cinema 1895-1939. Montreal: McGill-Queen's University Press, 1978.

Mebold, Anke and Charles Tepperman. "Resurrecting the lost history of $28 \mathrm{~mm}$ film in North America." Film History 15, no. 2 (January 2003): 137-151.

Pierce, David. "The Legion of the Condemned- Why American Silent Films Perished." Film History 9, no. 1. (1997), 5-22

Pratley, Gerald. "Torn Sprockets: The Uncertain Projections of the Canadian Film. Toronto: Associated University Presses, 1987.

Rossell, Deac. "Amateur Cinema." In Encyclopedia of Early Cinema, edited by Richard Abel, 17-18. London: Routledge, 2005. https://muse-jhu-edu.ezproxy.lib.ryerson.ca/article/235600/pdf

Singer, Ben. "Early Home Cinema and The Edison Home Projecting Kinetoscope." Film History Vol. 2. (1988): 37-69. http://www.jstor.org/stable/3814949

Wozny, Michele. "National Audiovisual Preservation Initiatives and the Independent Media Arts in Canada." Archivaria 67 (Spring 2009): 87-113.

https://archivaria.ca/index.php/archivaria/article/view/13210/14488

Websites

“Cinemachinery for the Personal Movie." Cinematographic Annual 1, (1930): 513-539. https://muse-jhu-edu.ezproxy.lib.ryerson.ca/article/235600/pdf

“The New Primer Pathéscope," Arts and Decoration, September, 1919, 248, https://archive.org/details/artsdecoration11newy/page/248

Library and Archives Canada. "Preservation Activities." Accessed May 12, 2019. http://www.bac-lac.gc.ca/eng/about-us/preservation/Pages/preservation-activities.aspx\#g

Library and Archives Canada. "Search-Film, Video and Sound." Accessed May 4, 2019, http://www.bac-lac.gc.ca/eng/discover/films-videos-sound-recordings/film-video-sound-

Library and Archives Canada. "Graphic Consultants Fonds." Accessed 25 May 2019, http://collectionscanada.gc.ca/pam archives/index.php?fuseaction=genitem.displayItem\&rec $\underline{\text { nbr }=190826 \& l a n g=e n g \& r e c \_n b r \_ \text {list }=190826,105494,140416,98129,4238700,105872,48161}$ $\underline{92,106871,106735,106723}$ 
Library and Archives Canada. "Search-Film, Video, and Sound database." Accessed 2 June 2019, http://www.bac-lac.gc.ca/eng/discover/films-videos-sound-recordings/film-videosound-database/Pages/search.aspx

Library and Archives Canada. " $28 \mathrm{~mm}$ - Film, Video, and Sound database results." Accessed 2 June 2019, http://www.bac-lac.gc.ca/eng/discover/films-videos-sound-recordings/film-video$\underline{\text { sound-database/Pages/list.aspx?k=28mm\&MediaEn=Film\& }}$

Library and Archives Canada. "28mm - Collection Search - Film, Video, and Sound database results." Accessed 2 june 2019, http://www.baclac.gc.ca/eng/collectionsearch/Pages/collectionsearch.aspx?DataSource=Archives|FilVidAnd $\underline{\text { Sou } \& \mathrm{q}=28 \mathrm{~mm} \& O \text { perator } 1=\text { AND\&Operator } 2=\text { AND\&MediaCode }=\mathrm{F} \&}$

Library and Archives Canada. "Wool from the Fleece to the Finished Product: [incomplete]." Accessed 13 June 2019, http://www.bac-lac.gc.ca/eng/discover/films-videos-soundrecordings/film-video-sound-database/Pages/item.aspx?IdNumber=416334

Library and Archives Canada. "Canadian National Exhibition: [incomplete].” Accessed 13 June 2019, http://www.bac-lac.gc.ca/eng/discover/films-videos-sound-recordings/film-videosound-database/Pages/list.aspx?IdNumber=460334\&

Library and Archives Canada. "Ayrshires at the Canadian National Exhibition, Toronto. ”Accessed 13 June 2019, http://www.bac-lac.gc.ca/eng/discover/films-videos-soundrecordings/film-video-sound-database/Pages/item.aspx?IdNumber=93350

Library and Archives Canada. "With the Plowmen at Brampton." Accessed, June 3 2019, http://www.bac-lac.gc.ca/eng/discover/films-videos-sound-recordings/film-video-sounddatabase/Pages/item.aspx?IdNumber $=192040$

Library and Archives Canada. "Project Naming." Accessed June 3, 2019, http://www.baclac.gc.ca/eng/discover/aboriginal-heritage/project-naming/Pages/introduction.aspx

Library and Archives Canada "We are Here sharing Stores." Accessed June 3, 2019, http://www.bac-lac.gc.ca/eng/discover/aboriginal-heritage/initiatives/Pages/stories.aspx 